\title{
Sparse graphs: metrics and random models
}

\author{
Béla Bollobás*†‡ Oliver Riordan*§
}

14th August, 2007

\begin{abstract}
Recently, Bollobás, Janson and Riordan introduced a very general family of random graph models, producing inhomogeneous random graphs with $\Theta(n)$ edges. Roughly speaking, there is one model for each kernel, i.e., each symmetric measurable function from $[0,1]^{2}$ to the non-negative reals, although the details are much more complicated, to ensure the exact inclusion of many of the recent models for large-scale real-world networks.

A different connection between kernels and random graphs arises in the recent work of Borgs, Chayes, Lovász, Sós, Szegedy and Vesztergombi. They introduced several natural metrics on dense graphs (graphs with $n$ vertices and $\Theta\left(n^{2}\right)$ edges), showed that these metrics are equivalent, and gave a description of the completion of the space of all graphs with respect to any of these metrics in terms of graphons, which are essentially kernels. One of the most appealing aspects of this work is the message that sequences of inhomogeneous quasi-random graphs are in a sense completely general: any sequence of dense graphs contains such a subsequence. Alternatively, their results show that certain natural models of dense inhomogeneous random graphs (one for each kernel) cover the space of dense graphs: there is one model for each point of the completion, producing graphs that converge to this point.

Our aim here is to investigate to what extent the results above for dense graphs can be generalized to graphs with $o\left(n^{2}\right)$ edges. Although many of the definitions extend in a simple way, the connections between the various metrics, and between the metrics and random graph models, turn out to be much more complicated than in the dense case. We shall prove many partial results, and state even more conjectures and open problems, whose resolution would greatly enhance the currently rather unsatisfactory theory of metrics on sparse graphs.
\end{abstract}

* Department of Pure Mathematics and Mathematical Statistics, Wilberforce Road, Cambridge CB3 0WB, UK

${ }^{\dagger}$ Department of Mathematical Sciences, University of Memphis, Memphis TN 38152, USA

$¥$ Research supported in part by NSF grants CCR-0225610 and DMS-0505550 and ARO grant W911NF-06-1-0076

$\S$ Research supported by a Royal Society Research Fellowship 


\section{Contents}

1 Introduction

2 Dense graphs 5

2.1 The subgraph distance . . . . . . . . . . . . . . 5

2.2 The cut distance . . . . . . . . . . . . . . . . . . . . . 8

2.3 Kernels and (quasi-)random graphs . . . . . . . . . . . . . 10

2.4 Equivalent kernels . . . . . . . . . . . . . . . . . . . . . . . 10

3 Subgraph counts for sparse graphs

3.1 Bounded and unbounded kernels . . . . . . . . . . . . . . 18

3.2 Non-uniform random graphs . . . . . . . . . . . . . . . . . . 20

3.3 Subgraph counts in the uniform case . . . . . . . . . . . . . 21

3.4 Partial results in the uniform case . . . . . . . . . . 27

3.5 Extensions to smaller $p \ldots \ldots \ldots \ldots$. . . . . . . . . . . . . . .

4 Szemerédi's Lemma and the cut metric 32

4.1 Weakly regular partitions . . . . . . . . . . . . 34

4.2 Strongly regular partitions . . . . . . . . . . . . . . . 36

4.3 Szemerédi's lemma and convergence in the cut norm . . . . . . 39

5 Comparison between cut and count convergence 43

5.1 Admissible subgraphs and their counts . . . . . . . . . . 43

5.2 Conjectured equivalence between cut and count convergence . . . 47

5.3 Partial results: embedding lemmas . . . . . . . . . . . . . . 50

5.4 Embeddings or homomorphisms? . . . . . . . . . . . . . 61

6 The extremely sparse case 67

6.1 The cut metric and Szemerédi's Lemma . . . . . . . . . . . 68

6.2 Tree counts . . . . . . . . . . . . . . . . . . . 74

6.3 Tree counts in random graphs . . . . . . . . . . . . 78

6.4 The partition metric . . . . . . . . . . . . 87

6.4.1 The partition metric in the denser setting. . . . . . . . . . 91

6.4.2 The partition metric and random graphs . . . . . . . . . 93

6.5 Which kernels give the same random graphs? . . . . . . . . . . 95

6.6 General extremely sparse graphs . . . . . . . . . . . . 97

7 Further metrics, models and questions $\quad 100$

7.1 The coloured neighbourhood metric for extremely sparse graphs . 100

7.2 Models for metrics . . . . . . . . . . . . . . . . . . . . . 101

7.3 Subgraph counts revisited . . . . . . . . . . . . . . 103

8 Closing Remarks 104 


\section{Introduction}

In recent years, much work has been done constructing and analyzing mathematical models of real-world networks. The random graphs in these models are inhomogeneous - in fact, many of them have degree sequences with power law distributions. In [10] Bollobás, Janson and Riordan defined a very general model of an $n$-vertex random graph $G(n, \kappa)$ with conditional independence between the edges which includes exactly many of the models of real-world graphs that have been studied, and proved numerous results about the random graphs generated by this model, including results about their component structure and the point and nature of the phase transition in them. Here the kernel $\kappa$ is a symmetric measurable function from $[0,1]^{2}$ to $[0, \infty)$ satisfying some mild conditions. (Some of these conditions arise due to the very general nature of other parts of the model, and can be weakened in other contexts; see [11 for a discussion of this.) Just like the real-world graphs that motivated the construction of the BJR model, the random graphs $G(n, \kappa)$ are sparse in the sense that the expected number of edges is $O(n)$ (in fact, $(c+o(1)) n$ for some constant $c$ ). In [10] the kernel $\kappa$ was used to define a multitype branching process $\mathcal{X}_{\kappa}$ whose survival probability is closely related to the component structure of $G(n, \kappa)$.

In order to decide how well our random graph $G(n, \kappa)$ approximates a given real-world graph $G_{n}$, it would be desirable to establish a distance between a random graph model and a graph, so that the approximation is judged to be better and better as the distance tends to 0 . Putting it slightly differently, we should like to define a metric on the set of sparse finite graphs so that a Cauchy sequence consists of graphs that are in some sense 'similar', and the limit of such a (not eventually constant) sequence is naturally identified with a suitable random graph model. For dense graphs, graphs with $n$ vertices and at least $\mathrm{cn}^{2}$ edges, such a program has been carried out very successfully in a series of papers by (various subsets of) Borgs, Chayes, Lovász, Sós, Szegedy and Vesztergombi (see [15, 16, 35, 36, 17, 18 and the references therein). In particular, they introduced several metrics on the space of dense finite (weighted) graphs and showed them to be equivalent. The limiting objects, i.e., the additional points in the completion, turn out to be graphons, that is, bounded symmetric measurable functions from $[0,1]^{2}$ to $\mathbb{R}$. The corresponding random graph models are the natural dense version of $G(n, \kappa)$, called $W$-random graphs in 35 .

The appearance of graphons or kernels (which are essentially equivalent) in the two different contexts described above suggests the existence of interesting connections between these areas. One such connection is described by Bollobás, Borgs, Chayes and Riordan [9], who study (sparse) random subgraphs of arbitrary dense graphs.

Our aim in this paper is to take the first tentative steps towards a general theory of metrics on sparse graphs; in particular, we shall investigate to what extent the ideas of Borgs, Chayes, Lovász, Sós, Szegedy and Vesztergombi can be carried over to the sparse setting, and what can be said about the connection between the metrics and the ideas of Bollobás, Janson and Riordan. As we shall see, the difficulties that arise are considerably greater than in the dense case; 
in fact, the difficulties increase as the graphs get sparser. The 'almost dense case', with $e\left(G_{n}\right)=n^{2-o(1)}$, is already rather different from the dense case, but the 'extremely sparse' case, with $e\left(G_{n}\right)=\Theta(n)$, is very different indeed, having many novel features. We shall prove numerous results, but the picture we obtain is much less complete than that obtained by Borgs et al in the dense case. In fact, the paper can be viewed as an attempt to identify some of the main problems and conjectures whose resolution would enhance the theory of metrics on sparse graphs.

An important tool in the study of metrics on spaces of dense graphs is Szemerédi's Regularity Lemma. While there is a version of Szemerédi's Lemma for sparse graphs (with $o\left(n^{2}\right)$ but $\omega(n)$ edges) satisfying a mild additional condition, there is no satisfactory counting/embedding lemma for counting (or even finding) small subgraphs using regular partitions. This is one of the reasons why sparse graphs are much more difficult to handle than dense ones. One of our main aims is to prove such a counting lemma for certain subgraphs, greatly extending a result of Chung and Graham 19.

The rest of the paper is organized as follows. The next section is about dense graphs and kernels; we start by briefly recalling some of the definitions and results of Borgs, Chayes, Lovász, Sós, Szegedy and Vesztergombi whose generalization we shall discuss. Then, in Subsection 2.4 we prove a characterization of when two kernels are equivalent; we shall need this when we come to sparse graphs.

The rest of the paper concerns sparse graphs, i.e., graphs with $n$ vertices and $o\left(n^{2}\right)$ edges: in Section 3 we consider subgraph counts in sparse (but mostly not too sparse) graphs, stating a conjecture that generalizes the main result of Lovász and Szegedy [35, and proving various partial results, concentrating especially on the uniform case, i.e., on sparse quasirandom graphs. In Section 4 we turn to Szemerédi's Lemma for sparse graphs satisfying an appropriate 'bounded density' assumption, and the consequences for questions of convergence in the cut metric.

Sections 5 and 6 are the longest and most important sections of our paper. In Section 5 we discuss the relationship between the cut and count metrics in the sparse case. As well as proposing various conjectures extending the results of Borgs, Chayes, Lovász, Sós and Vesztergombi, we prove several partial results, amounting to 'sparse counting lemmas' with various assumptions; these results, Theorem 5.13 and its variants Theorems 5.14 and 5.16, are the most substantial results in the paper.

In Section 6 we turn to the extremely sparse case, considering graphs with bounded average degree. In this setting the cut metric makes very little sense, and essentially none of the results for denser graphs carry over. In fact, several new phenomena arise, leading to many interesting questions, such as, for which kernels $\kappa_{1}, \kappa_{2}$ are the BJR models $G\left(n, \kappa_{1}\right)$ and $G\left(n, \kappa_{2}\right)$ essentially equivalent? Even in the subcritical case when all components are 'small', this question is still interesting: it then reduces to a question about multi-type Poisson branching processes. In the finite-type case, we prove two equivalent characterisations of the set of pairs of kernels giving rise to multi-type branching processes that are 
the same when seen without types (see Theorems 6.8 and 6.9). We also discuss metrics capturing (separately) the local and global behaviour of extremely sparse graphs; these seem much harder to relate than in the denser setting.

In Section 7 we return to the relationship between metrics and random graph models, proposing various new metrics (including one that combines local and global information in extremely sparse graphs) and various new random graph models; this section, and indeed the paper as a whole, is rather open ended. We close with some final remarks in Section 8

\section{Dense graphs}

There are many natural definitions of what it means for two graphs to be 'close', and corresponding metrics and notions of Cauchy/fundamental sequences. These tend to be particularly natural for 'dense' graphs, with $\Theta\left(n^{2}\right)$ edges. Several of these metrics have been studied by Borgs, Chayes, Lovász, Sós and Vesztergombi [17, 18, who showed that they are equivalent, and that there is a natural completion of the space of graphs under any of these metrics. In this section we briefly recall some of these definitions and results; we are not aiming to survey the results of these papers, only those that will be relevant for us here. Although most of the results mentioned in Subsections 2.1 2.3 will be from Lovász and Szegedy [35] and [17, 18, we shall not always adopt their notation or terminology, or indeed follow their definitions exactly.

Borgs, Chayes, Lovász, Sós and Vesztergombi [17, 18, consider weighted graphs, with weights on the edges and on the vertices. For the results we shall describe, this makes essentially no difference. In what follows, we consider only unweighted graphs; while much of what we shall say presumably carries over to suitably weighted graphs, the definitions for weighted graphs are not as natural in the sparse case, and are likely to introduce more additional complications than new insights.

\subsection{The subgraph distance}

The basic starting point is to consider, for each fixed graph $F$, the number of copies of $F$ in a large graph $G$, i.e., the number $X_{F}(G)$ of subgraphs of $G$ isomorphic to $F$. Although $X_{F}(G)$ (for example, the number of triangles in $G$ ) is the most natural basic notion in this context, it turns out to be cleaner to work with something slightly different, counting copies of $F$ seen as a graph with labelled vertices. In other words, we consider $\operatorname{emb}(F, G)$, the number of embeddings of $F$ into $G$, i.e., the number of injections $\phi$ from $V(F)$ to $V(G)$ with the property that $\phi(x) \phi(y)$ is an edge of $G$ whenever $x y \in E(F)$. (This is also known as the number of injective homomorphisms from $F$ into $G$.) Note that

$$
\operatorname{emb}(F, G)=\operatorname{aut}(F) X_{F}(G),
$$

so $X_{F}(G)$ and $\operatorname{emb}(F, G)$ contain the same information. Working with the latter avoids constant factors aut $(F)$ in many formulae. 
If $F$ has $k$ vertices, then for $n \geq k$ we have $\operatorname{emb}\left(F, K_{n}\right)=n_{(k)}=n(n-$ 1) $\cdots(n-k+1)$, so the natural normalization is to work with

$$
s(F, G)=\frac{\operatorname{emb}(F, G)}{n_{(k)}}=\frac{X_{F}(G)}{X_{F}\left(K_{n}\right)} \in[0,1],
$$

where, as usual $n=|G|$ is the number of vertices of $G$. If $|F|>|G|$ then the above ratio is not defined, and we set $s_{F}(G)=0$.

Let $\mathcal{F}$ denote the set of isomorphism classes of finite graphs; sometimes it will be convenient to enumerate $\mathcal{F}$ in an arbitrary way, writing $\mathcal{F}=\left\{F_{1}, F_{2}, \ldots\right\}$. The graph parameters $s(F, \cdot), F \in \mathcal{F}$, define a natural family of equivalent metrics on $\mathcal{F}$, by mapping $\mathcal{F}$ into $[0,1]^{\infty}$ (or into $[0,1]^{\mathcal{F}}$ ). Indeed, for any finite graph $G$, set

$$
s(G)=\left(s_{i}(G)\right)_{i=1}^{\infty} \in[0,1]^{\infty},
$$

where $s_{i}(G)=s\left(F_{i}, G\right)$. Let $d$ be any metric on $X=[0,1]^{\infty}$ which gives the product topology, for example $d(s, t)=\sum_{i=1}^{\infty} 2^{-i}\left|s_{i}-t_{i}\right|$. We may define the subgraph distance of two graphs $G_{1}, G_{2}$ as

$$
d_{\mathrm{sub}}\left(G_{1}, G_{2}\right)=d\left(s\left(G_{1}\right), s\left(G_{2}\right)\right) .
$$

It is easy to see that this defines a metric on $\mathcal{F}$ : indeed, given $G \in \mathcal{F}$, among graphs $F$ with $s(F, G)>0$, there is a unique graph with $|F|+e(F)$ maximal, namely $G$. Thus, the map $G \mapsto s(G)$ is injective. Furthermore, considering $s\left(E_{n+1}, G\right)$, where $E$ is the empty graph with $n+1$ vertices, we see that the distance between any graph $G$ with $n$ vertices and the set of graphs with more than $n$ vertices is positive. It follows that the metric space $\left(\mathcal{F}, d_{\text {sub }}\right)$ is discrete.

A sequence $\left(G_{n}\right)$ of graphs is Cauchy with respect to $d_{\text {sub }}$ if and only if, for each $F \in \mathcal{F}$, the sequence $s(F, G)$ converges. Such sequences are sometimes called 'convergent', although they do not converge in the metric space $\left(\mathcal{F}, d_{\text {sub }}\right)$. Note that if $\left(G_{n}\right)$ is Cauchy then, since $\left(\mathcal{F}, d_{\text {sub }}\right)$ is discrete, either $\left(G_{n}\right)$ is eventually constant, or $\left|G_{n}\right| \rightarrow \infty$.

Many minor variations on the definition of $d_{\text {sub }}$ are possible. For example, instead of considering the number of embeddings of $F$ into $G$, one can consider the number $\operatorname{hom}(F, G)$ of homomorphisms from $F$ to $G$, i.e., of maps $\phi: V(F) \rightarrow$ $V(G)$ such that $\phi(x) \phi(y) \in E(G)$ whenever $x y \in E(F)$. If $|F|=k$, then the number of non-injective homomorphisms from $F$ to $G$ is at most $\left(\begin{array}{c}k \\ 2\end{array}\right) n^{k-1}=$ $o\left(n^{k}\right)$, so setting

$$
t(F, G)=\operatorname{hom}(F, G) / n^{k}
$$

we have $t(F, G)=s(F, G)+o(1)$ when $F$ is fixed and $|G| \rightarrow \infty$. Hence, in this dense case, the parameters $s(F, \cdot)$ and $t(F, \cdot)$ are essentially equivalent. [There is a minor difference that, working with homomorphisms, one ends up with a pseudo-metric: if $G$ is any graph and $G^{(r)}$ is the blow-up of $G$ obtained by making $r$ copies of each vertex, joined to all copies of its neighbours, then $t\left(F, G^{(r)}\right)=t(F, G)$ for all $F \in \mathcal{F}$ and $r \geq 1$.] Also, one can pass easily back and forth between subgraph counts and counts of induced subgraphs using inclusion-exclusion. 
One of the key properties of the metric $d_{\text {sub }}$ is that there is a natural description of the (clearly compact) completion of $\left(\mathcal{F}, d_{\text {sub }}\right)$, in terms of kernels (also called graphons). Here a kernel is a symmetric measurable function from $[0,1]^{2}$ to $[0,1]$. One can extend the definition of $s(F, G)$ (or of $t(F, G)$ ) to kernels in a natural way: given a finite graph $F$ with vertex set $\{1,2, \ldots, k\}$, let

$$
s(F, \kappa)=\int_{[0,1]^{k}} \prod_{i j \in E(F)} \kappa\left(x_{i}, x_{j}\right) \prod_{i=1}^{k} \mathrm{~d} x_{i} .
$$

(This is often denoted $t(F, \kappa)$.) This formula has a natural interpretation as the normalized 'number' of embeddings of $F$ into a weighted graph with the uncountable vertex set $[0,1]$, with edge weights given by $\kappa$. Of course, in this context there is no difference between embeddings and homomorphisms.

Lovász and Szegedy [35] proved (essentially) the following result.

Theorem 2.1. Let $\left(G_{n}\right)$ be a Cauchy sequence in $\left(\mathcal{F}, d_{\text {sub }}\right)$. Then either $\left(G_{n}\right)$ is eventually constant, or there is a kernel $\kappa$ such that $s\left(F, G_{n}\right) \rightarrow s(F, \kappa)$.

(The result proved in 35] concerns $t$ rather than $s$, which makes no difference, except that a separate case for eventually constant sequences is then not needed. Here, the distinction is informative: considering the parameters $s\left(E_{k}, G_{n}\right)$ for each $k$ shows that in the second case above we have $\left|G_{n}\right| \rightarrow \infty$.)

Of course, (11) allows one to extend the metric $d_{\text {sub }}$ to kernels, obtaining in the first instance a pseudo-metric on the set of kernels. There is a natural notion of equivalence for kernels, which one can think of as a two dimensional version of the equivalence relation on random variables given by $X \sim Y$ if $X$ and $Y$ have the same distribution; the details are somewhat technical, and not essential for understanding the metrics discussed here, so we postpone them to Subsection 2.4. We write $\sim$ for this relation, and $\mathcal{K}$ for the set of equivalence classes. It turns out that $\kappa_{1} \sim \kappa_{2}$ if and only if $d_{\text {sub }}\left(\kappa_{1}, \kappa_{2}\right)=0$ (see Theorem 2.6), so $d_{\text {sub }}$ induces a metric on $\mathcal{K}$. The metric space $\left(\mathcal{K}, d_{\text {sub }}\right)$ is complete (the result about Cauchy sequences of graphs above applies just as well to kernels $)$. Hence, the completion of $\left(\mathcal{F}, d_{\text {sub }}\right)$ is obtained by adding to $\mathcal{F}$ the set $\mathcal{K}$ of all equivalence classes of kernels, and using the map $s: \mathcal{F} \cup \mathcal{K} \rightarrow[0,1]^{\infty}$ to extend $d_{\text {sub }}$ to $\mathcal{F} \cup \mathcal{K}$.

There is a natural way to associate a kernel $\kappa_{G}$ to a graph $G$ with $n$ vertices: divide $[0,1]$ into $n$ intervals $I_{1}, \ldots, I_{n}$ of equal length (we may and shall ignore the question of which endpoints are included), and set $\kappa_{G}$ to be 1 on $I_{i} \times I_{j}$ if $i j \in E(G)$, and 0 otherwise. One slight advantage of using $t$ rather than $s$ is that $t(F, G)=s\left(F, \kappa_{G}\right)$ holds exactly for all graphs $F$ and $G$. However, the metric obtained using $t$ is only a pseudo-metric, since graphs on different numbers of vertices may correspond to the same kernel, for example if one is a blow-up of the other.

We say that a kernel $\kappa$ is of finite type if there is a partition of $[0,1]$ into measurable sets $A_{1}, \ldots, A_{k}$ so that $\kappa$ is constant on each of the rectangles $A_{i} \times$ $A_{j}$. Note that $\kappa_{G}$ is always of finite type - in fact, finite-type kernels correspond to weighted graphs, with weights on the vertices and the edges. 


\subsection{The cut distance}

Borgs, Chayes, Lovász, Sós and Vesztergombi 17] considered another natural metric on graphs or kernels, namely, the cut metric, based on a norm used by Frieze and Kannan [27. For any bounded measurable function $\kappa:[0,1]^{2} \rightarrow \mathbb{R}$, its cut norm $\|\kappa\|_{\text {cut }}$ is defined by

$$
\|\kappa\|_{\text {cut }}=\sup _{S, T \subset[0,1]}\left|\int_{S \times T} \kappa(x, y) d x d y\right|,
$$

where the supremum is over all pairs of measurable subsets of $[0,1]$. It is easily seen that this does define a norm on $L_{\infty}\left([0,1]^{2}\right)$. In fact, there are variations of this definition: one can take

$$
\|\kappa\|_{\text {cut }}=\sup _{S \subset[0,1]}\left|\int_{S \times S^{\mathrm{c}}} \kappa(x, y) d x d y\right|,
$$

where $S^{\mathrm{c}}=[0,1] \backslash S$, or one can take the supremum in (2) only over sets $S, T$ with with $S \cap T=\emptyset$. It is easy to check that these variations only affect the norm up to an (irrelevant) constant factor (see [17), so we shall feel free to use whichever definition is most convenient in any given context.

Before turning to the cut metric we need one further definition. Given a kernel $\kappa$ and a measure-preserving map $\tau:[0,1] \rightarrow[0,1]$, let $\kappa^{(\tau)}$ be the kernel defined by

$$
\kappa^{(\tau)}(x, y)=\kappa(\tau(x), \tau(y)) .
$$

If $\tau$ is a bijection, then we call $\tau$ a rearrangement (of $[0,1]$ ), and $\kappa^{(\tau)}$ a rearrangement of $\kappa$. (It is perhaps more natural to consider measure-preserving bijections between two subsets of $[0,1]$ with measure 1 ; this makes no difference.) Two kernels $\kappa_{1}$ and $\kappa_{2}$ are naively equivalent if one is a rearrangement of the other, more precisely, if there is a rearrangement $\tau$ such that

$$
\kappa_{1}(x, y)=\kappa_{2}^{(\tau)}(x, y) \quad \text { a.e. }(x, y) \in[0,1]^{2} .
$$

In this case we write $\kappa_{1} \approx \kappa_{2}$, noting that $\approx$ is an equivalence relation.

The cut metric $d_{\text {cut }}$ on the set of kernels may be defined as follows:

$$
d_{\text {cut }}\left(\kappa_{1}, \kappa_{2}\right)=\inf _{\kappa_{2}^{\prime} \approx \kappa_{2}}\left\|\kappa_{1}-\kappa_{2}^{\prime}\right\|_{\text {cut }} .
$$

Clearly, this defines a pseudo-metric on kernels; in particular, if $\kappa_{1} \approx \kappa_{2}$, then $d_{\text {cut }}\left(\kappa_{1}, \kappa_{2}\right)=0$. The reverse implication does not hold; in fact, $d_{\text {cut }}\left(\kappa_{1}, \kappa_{2}\right)=0$ if and only if $\kappa_{1} \sim \kappa_{2}$, where $\sim$ is the equivalence relation to be defined in Subsection 2.4. Hence, $d_{\text {cut }}$ induces a metric on the set $\mathcal{K}$ of equivalence classes of $\sim$.

As noted above, there is a kernel $\kappa_{G}$ naturally associated to each graph $G$, although the map $G \mapsto \kappa_{G}$ from $\mathcal{F}$ to $\mathcal{K}$ is not injective. We extend the cut metric to a pseudo-metric on graphs by setting

$$
d_{\text {cut }}\left(G_{1}, G_{2}\right)=d_{\text {cut }}\left(\kappa_{G_{1}}, \kappa_{G_{2}}\right),
$$


and to $\mathcal{F} \cup \mathcal{K}$ similarly.

For graphs $G_{1}, G_{2}$ on $n$ vertices, there is a much more natural variant of their cut distance: let $\widehat{d}_{\text {cut }}\left(G_{1}, G_{2}\right)$ be the smallest $\varepsilon$ for which we can identify the vertices of $G_{1}$ with those of $G_{2}$ such that for any bipartition of the vertex set, the corresponding cuts in $G_{1}$ and $G_{2}$ have sizes within $\varepsilon n^{2}$. In terms of kernels,

$$
\widehat{d}_{\text {cut }}\left(G_{1}, G_{2}\right)=\min _{\kappa \approx_{n} \kappa_{G_{2}}}\left\|\kappa_{G_{1}}-\kappa\right\|_{\text {cut }},
$$

where $\kappa_{1} \approx_{n} \kappa_{2}$ if (5) holds for some map $\tau$ that simply permutes the intervals $I_{n}$ corresponding to the vertices, and we take (3) as the definition of the cut norm. Note that the supremum appearing in the cut norm in (8) is over all bipartitions of $[0,1]$, not just those corresponding to bipartitions of the vertices; it is very easy to see that this makes no difference: the supremum is attained at a vertex bipartition.

Comparing (7) and (8), since the infimum in the former is taken over a larger set, one trivially has $d_{\text {cut }}\left(G_{1}, G_{2}\right) \leq \widehat{d}_{\text {cut }}\left(G_{1}, G_{2}\right)$. Borgs, Chayes, Lovász, Sós and Vesztergombi [17 noted that strict inequality is possible. For example, taking (3) as the definition of the cut norm, let $G_{1}$ be a triangle, and let $G_{2}$ be the graph with 3 vertices and one edge. For any pairing of the vertices, there is a cut with two more edges in $G_{1}$, so $\widehat{d}_{\text {cut }}\left(G_{1}, G_{2}\right)=2 / 9$. On the other hand, consider the blowups $G_{1}^{(2)}$, a complete tripartite graph, and $G_{2}^{(2)}$, a $C_{4}$ with two isolated vertices added. Placing two opposite vertices of the $C_{4}$ in one class of $G_{1}^{(2)}$, and the other vertices in different classes, we realize $G_{2}^{(2)}$ as a subgraph of $G_{1}^{(2)}$ in such a way that the 8 edges of $G_{1}^{(2)}$ not present in $G_{2}^{(2)}$ form a non-bipartite graph, so no cut cuts more than 7 of these edges. It follows that

$$
d_{\text {cut }}\left(G_{1}, G_{2}\right) \leq \widehat{d}_{\text {cut }}\left(G_{1}^{(2)}, G_{2}^{(2)}\right) \leq 7 / 6^{2}<2 / 9 .
$$

(In fact, the rearrangement described gives 6/36 as an upper bound.) For questions of convergence, however, the two notions are equivalent: as shown in [17,

$$
d_{\text {cut }}\left(G_{1}, G_{2}\right) \leq \widehat{d}_{\text {cut }}\left(G_{1}, G_{2}\right) \leq 32 d_{\text {cut }}\left(G_{1}, G_{2}\right)^{1 / 67} .
$$

One of the main results of Borgs, Chayes, Lovász, Sós and Vesztergombi, namely Theorem 2.6 in 17, is that the metrics $d_{\text {sub }}$ (defined using $t$ rather than $s)$ and $d_{\text {cut }}$ are equivalent: $\left(G_{n}\right)$ is a Cauchy sequence for $d_{\text {sub }}$ if and only if it is a Cauchy sequence for $d_{\text {cut }}$. In the light of the various other results of Lovász and Szegedy 35 and Borgs, Chayes, Lovász, Sós and Vesztergombi 17, this statement may be reformulated in our notation as follows.

Theorem 2.2. Let $\left(G_{n}\right)$ be a sequence of graphs or kernels with $\left|G_{n}\right| \rightarrow \infty$, where we take $\left|G_{n}\right|=\infty$ if $G_{n}$ is a kernel, and let $\kappa$ be a kernel. Then $d_{\text {sub }}\left(G_{n}, \kappa\right) \rightarrow 0$ if and only if $d_{\text {cut }}\left(G_{n}, \kappa\right) \rightarrow 0$.

An immediate consequence of this result is the following, Corollary 3.10 in [17. 
Corollary 2.3. Let $\kappa$ and $\kappa^{\prime}$ be two kernels. Then $s(F, \kappa)=s\left(F, \kappa^{\prime}\right)$ for every $F$ if and only if $d_{\text {cut }}\left(\kappa, \kappa^{\prime}\right)=0$.

We shall return to a discussion of kernels at cut distance 0 shortly.

\subsection{Kernels and (quasi-)random graphs}

As well as going from graphs to kernels, one can go from kernels to (random) graphs in a very natural way, as in Section 2.6 of Lovász and Szegedy [35], or as in Bollobás, Janson and Riordan [10 for the sparse case. Indeed, given a kernel $\kappa$ and an $n \geq 1$, let $G(n, \kappa)$ be the random graph on $[n]$ defined as follows: first let $x_{1}, \ldots, x_{n}$ be iid with the uniform distribution on $[0,1]$. Given the $x_{i}$, join each pair of vertices independently, joining $i$ and $j$ with probability $\kappa\left(x_{i}, x_{j}\right)$. The resulting graph is called a $\kappa$-random graph by Lovász and Szegedy [35, although they use $W$ as their default symbol for a kernel. It is easy to check that, for each $F$, the random variable $s(F, G(n, \kappa))$ converges (in probability and in fact almost surely) to $s(F, \kappa)$ as $n \rightarrow \infty$. Thus the sequence $G(n, \kappa)$ converges almost surely to $\kappa$ in the metric $d_{\text {sub }}$ or $d_{\text {cut }}$.

It is natural to view a sequence $\left(G_{n}\right)$ converging to $\kappa$ in $d_{\text {sub }}$ as a sequence of 'inhomogeneous quasi-random graphs': when $\kappa$ is constant, the convergence condition is equivalent to the standard notion of quasi-randomness, introduced by Thomason [40] in 1987 (although he called it pseudo-randomness) and studied in great detail by Chung, Graham and Wilson [20] and many others. The convergence of $G(n, \kappa)$ to $\kappa$ in $d_{\text {sub }}$ establishes that sequences generated by the natural inhomogeneous random model are also quasi-random, as one would hope. One of the most pleasing features of this whole subject area is the interpretation that inhomogeneous quasi-random graphs are completely general: any sequence of (dense) graphs has such a subsequence.

To take an alternative viewpoint, we may think of kernels as uncountable infinite graphs, and a 'typical' random graph $G(n, \kappa)$ as a good finite approximation to $\kappa$. Then the completion of $\mathcal{F}$ is obtained by adding these infinite graphs, and the approximations $G(n, \kappa)$ ( $n$ large) are examples of finite graphs close to a given infinite graph. Taking this viewpoint it is natural not to identify a finite graph with a kernel.

\subsection{Equivalent kernels}

In the light of Corollary 2.3, it is clearly important to understand which pairs of kernels have $d_{\text {cut }}\left(\kappa_{1}, \kappa_{2}\right)=0$; this is also important for understanding $d_{\text {cut }}$ itself. Fortunately, it turns that there is a natural notion of equivalence for kernels which gives the answer. Since this topic is only touched on in passing in Borgs, Chayes, Lovász, Sós and Vesztergombi [17, we shall go into some detail here.

Roughly speaking, we would like to say that two kernels are equivalent if one is obtained from the other simply by relabelling the 'types' in $[0,1]$. It would seem that the notion $\approx$ of naive equivalence defined in (5) is thus the right 
one, but a little thought shows that this is not the case; for this, the random viewpoint is very helpful.

So far, as in [17, we defined kernels only on $[0,1]^{2}$. In view of the connection to random graphs discussed in the previous subsection, it is a priori more natural to work with a general probability space $X=(\Omega, \mathcal{F}, \mu)$ rather than $[0,1]$ with Lebesgue measure, defining a kernel as a symmetric measurable function from the square of a probability space to $[0,1]$. (This is the approach taken in the sparse case by Bollobás, Janson and Riordan [10.) However, almost all the time, we shall consider only kernels on $[0,1]$; there are two reasons for doing so: firstly, graphs with $n$ vertices correspond to kernels on the discrete space with $n$ equiprobable elements, and $[0,1]$ is the natural limit of these spaces. Secondly, all probability spaces that one would ever wish to work with (all socalled 'standard' probability spaces) are isomorphic to Lebesgue measure on an interval, combined with (possibly) a finite or countable number of atoms. When studying kernels, the presence of atoms makes no difference: for example, a kernel on a finite measure space corresponds in a natural way to a piecewise constant kernel on $[0,1]$. Hence it makes very good sense to consider only kernels on $[0,1]$.

We may think of kernels as two-dimensional versions of random variables (not to be confused with vector valued random variables). Two random variables are equivalent if they have the same distribution. Equivalently, they are equivalent if they may be coupled so as to agree with probability 1 . This is the definition we shall use for kernels.

Working, for the moment, on general (standard) probability spaces, and suppressing the $\sigma$-field of measurable sets in the notation, let $\left(\Omega_{1}, \mu_{1}\right)$ and $\left(\Omega_{2}, \mu_{2}\right)$ be two probability spaces. A coupling of $\left(\Omega_{1}, \mu_{1}\right)$ and $\left(\Omega_{2}, \mu_{2}\right)$ is simply a probability space $(\Omega, \mu)$ together with measure-preserving maps $\sigma_{i}: \Omega \rightarrow \Omega_{i}, i=1,2$. Thus, if $X$ is a uniformly random point of $(\Omega, \mu)$, then $\sigma_{1}(X)$ and $\sigma_{2}(X)$ are uniform on $\left(\Omega_{1}, \mu_{1}\right)$ and $\left(\Omega_{2}, \mu_{2}\right)$, respectively. Let $\kappa_{i}$ be a kernel on $\left(\Omega_{i}, \mu_{i}\right)$, $i=1,2$. Then $\kappa_{1}$ and $\kappa_{2}$ are equivalent if there is a coupling of the underlying probability spaces such that

$$
\kappa_{1}\left(\sigma_{1}(x), \sigma_{1}(y)\right)=\kappa_{2}\left(\sigma_{2}(x), \sigma_{2}(y)\right) \text { for }(\mu \times \mu) \text {-almost every }(x, y) \in \Omega^{2} ;
$$

in other words, extending the notation in (4) to arbitrary spaces, we require $\kappa_{1}^{\left(\sigma_{1}\right)}=\kappa_{2}^{\left(\sigma_{2}\right)}$ a.e. We write $\sim$ for the corresponding relation. Although this definition may seem a little complicated, as explained above it is in fact very natural.

Note that $\kappa_{1} \approx \kappa_{2}$ implies $\kappa_{1} \sim \kappa_{2}$ : if $\kappa_{1}=\kappa_{2}^{(\tau)}$, then one couples $x \in$ $[0,1]=\Omega_{1}$ with $\tau(x) \in \Omega_{2}$. (More formally, we may take $\Omega=\Omega_{1}$, with $\sigma_{1}$ the identity and $\sigma_{2}=\tau$.) It is easy to see that the reverse implication does not hold: for example, consider the random variables $\Lambda_{1}, \Lambda_{2}$ on $[0,1]$ given by $\Lambda_{1}(x)=x$ and $\Lambda_{2}(x)=2 x-\lfloor 2 x\rfloor$; these both have the uniform distribution, but since one is 1-to- 1 and the other 2-to-1, there is no measure-preserving bijection from one ground space to the other transforming one into the other. Setting $\kappa_{i}(x, y)=\Lambda_{i}(x) \Lambda_{i}(y)$, one obtains kernels with $\kappa_{1} \sim \kappa_{2}$ but $\kappa_{1} \not \approx \kappa_{2}$. 
Returning to the special case of kernels on $[0,1]$, essentially equivalent to the general case, couplings have a very simple description. All that matters is that, for a uniform point $X$ of $(\Omega, \mu)$, the distribution of $\left(\sigma_{1}(X), \sigma_{2}(X)\right)$, which should have uniform marginals. Thus, couplings correspond to doubly stochastic measures, i.e., Borel measures $\mu$ on $[0,1]^{2}$ with both marginals Lebesgue measure. In other words, we have $\kappa_{1} \sim \kappa_{2}$ if and only if there is a doubly stochastic measure $\mu$ such that

$$
\kappa_{1}(x, y)=\kappa_{2}(u, v) \text { for }(\mu \times \mu) \text {-a.e. }(x, u, y, v) \in[0,1]^{4} .
$$

At first sight, $[0,1]^{2}$ is the most natural space to use to couple two kernels on $[0,1]$, but there is another natural choice. Since $[0,1]^{2}$ is isomorphic as a probability space to $[0,1]$, we may construct the coupling on $[0,1]$ ! Hence, $\kappa_{1} \sim \kappa_{2}$ if and only if there are measure preserving maps $\sigma_{1}, \sigma_{2}:[0,1] \rightarrow[0,1]$ such that $\kappa_{1}^{\left(\sigma_{1}\right)}=\kappa_{2}^{\left(\sigma_{2}\right)}$ for (Lebesgue) a.e. $(x, y) \in[0,1]^{2}$. Putting this a little more symmetrically, we see that $\kappa_{1} \sim \kappa_{2}$ if and only if

$$
\exists \kappa, \sigma_{1}, \sigma_{2} \text { such that } \kappa=\kappa_{1}^{\left(\sigma_{1}\right)} \text { a.e and } \kappa=\kappa_{2}^{\left(\sigma_{2}\right)} \text { a.e, }
$$

where $\kappa$ is a kernel on $[0,1]$ and $\sigma_{1}$ and $\sigma_{2}$ are measure-preserving maps from $[0,1]$ to itself. Note that $\kappa \sim \kappa^{(\sigma)}$ for any kernel $\kappa$ on $[0,1]$ and any measurepreserving map from $[0,1]$ to itself.

Since couplings rather than rearrangements give the proper notion of equivalence for two kernels, it is natural to use couplings rather than rearrangements in the definition of the cut metric. Indeed, Borgs, Chayes, Lovász, Sós and Vesztergombi [17 define the cut metric as follows:

$$
d_{\text {cut }}\left(\kappa_{1}, \kappa_{2}\right)=\inf _{\mu \in \mathcal{M}} \sup _{S, T}\left|\int_{S \times T}\left(\kappa_{1}(x, y)-\kappa_{2}(u, v)\right) d \mu(x, u) d \mu(y, v)\right|,
$$

where $\mathcal{M}$ is the set of doubly stochastic measures on $[0,1]^{2}, S$ and $T$ run over measurable subsets of $[0,1]^{2}$, and the integral is over $(x, u) \in S$ and $(y, v) \in T$. As shown in [17, the definitions (6) and (11) coincide. (This is not hard to see - either formula defines a function that is continuous, indeed Lipschitz with constant 1 , with respect to the cut norm, and hence continuous with respect to the $L_{1}$ norm. Since the finite-type kernels are dense in $L_{1}$, it suffices to check the equality of the two definitions for finite-type kernels, which is not hard. For the details, see [17.) Since (6) is much easier to work with than (11), we shall take the former as our definition of $d_{\text {cut }}$.

Although (6) is more convenient, there is a sense in which (11) is the 'right' definition. For example, as we shall now show, the infimum in (11) is always attained, unlike that in (6). This is not discussed in [17, where it is of no particular significance. Here, as in the bulk of the paper, unless otherwise specified, all kernels are kernels on $[0,1]$, i.e., symmetric Lebesgue-measurable functions $[0,1]^{2} \rightarrow[0,1]$. As noted above, it always suffices to consider kernels on $[0,1]$. 
Lemma 2.4. Let $\kappa_{1}$ and $\kappa_{2}$ be two kernels. Then there is a doubly stochastic measure $\mu$ achieving the infimum in (11).

Proof. For $\mu \in \mathcal{M}$ set

$$
d_{\mu}\left(\kappa_{1}, \kappa_{2}\right)=\sup _{S, T}\left|\int_{S \times T}\left(\kappa_{1}(x, y)-\kappa_{2}(u, v)\right) d \mu(x, u) d \mu(y, v)\right|,
$$

so our aim is to show that $\inf _{\mu \in \mathcal{M}} d_{\mu}\left(\kappa_{1}, \kappa_{2}\right)$ is attained. Before doing so, let us note that in the supremum one may restrict the sets $S$ and $T$ in (12) to 'nice' sets. Let $\mathcal{D}$ denote the set of finite unions of products of (half-open) intervals. Since $\mu$ is a finite Borel measure, for any measurable $S, T \subset[0,1]^{2}$ and any $\varepsilon>0$, there are sets $S^{\prime}, T^{\prime} \in \mathcal{D}$ with $\mu\left(S \Delta S^{\prime}\right), \mu\left(T \Delta T^{\prime}\right)<\varepsilon$. Since $\kappa_{1}-\kappa_{2}$ is bounded by \pm 1 , replacing $S, T$ by $S^{\prime}$ and $T^{\prime}$ changes the value of the integral by at most $2 \varepsilon$.

It is well known that $\mathcal{M}$ is compact in the topology in which $\mu_{n} \rightarrow \mu$ if and only if $\mu_{n}(A) \rightarrow \mu(A)$ for every set $A$ that is the product of two intervals, or, equivalently, for every $A \in \mathcal{D}$. This follows from weak-* compactness, approximating the characteristic function of $A$ suitably, and may also be seen by noting that each $\mu \in \mathcal{M}$ corresponds to a function (a 'copula') $C:[0,1]^{2} \rightarrow[0,1]$ given by $C(x, y)=\mu([0, x] \times[0, y])$, and that the set of functions $C$ satisfying suitable boundary and monotonicity conditions is in 1-to-1 correspondence with $\mathcal{M}$. Note that one cannot require $\mu_{n}(A) \rightarrow \mu(A)$ for every measurable $A$ : it is easy to construct sequences where the limiting measure is concentrated on, for example the diagonal $S=\{(x, x)\}$, with $\mu_{n}(S)=0$ for every $n$.

Let $\left(\mu_{n}\right)$ be a sequence of doubly stochastic measures with $d_{\mu_{n}}\left(\kappa_{1}, \kappa_{2}\right) \rightarrow$ $d_{\text {cut }}\left(\kappa_{1}, \kappa_{2}\right)$; such a sequence exists by the definition (11) of $d_{\text {cut }}\left(\kappa_{1}, \kappa_{2}\right)$. From the remark above, $\left(\mu_{n}\right)$ has a subsequence converging to some $\mu \in \mathcal{M}$ in the appropriate topology. Restricting to this subsequence, we may assume that $\mu_{n}(A) \rightarrow \mu(A)$ for every $A \in \mathcal{D}$.

Let $S=S_{1} \times S_{2}$ and $T=T_{1} \times T_{2}$, where $S_{1}, S_{2}, T_{1}$ and $T_{2}$ are all intervals in $[0,1]$. We claim that

$$
\int_{S \times T} \kappa(x, y) d \mu_{n}(x, u) d \mu_{n}(y, v) \rightarrow \int_{S \times T} \kappa(x, y) d \mu(x, u) d \mu(y, v)
$$

as $n \rightarrow \infty$, for any kernel $\kappa$. Before proving this, let us show that the lemma follows.

For any $\nu \in \mathcal{M}$, let

$$
f(S, T, \nu)=\int_{S \times T}\left(\kappa_{1}(x, y)-\kappa_{2}(u, v)\right) d \nu(x, u) d \nu(y, v),
$$

so $d_{\nu}\left(\kappa_{1}, \kappa_{2}\right)=\sup _{S, T} f(S, T, \nu)$. Applying (13) with $\kappa=\kappa_{1}$ and $\kappa=\kappa_{2}$, we see that $f\left(S, T, \mu_{n}\right) \rightarrow f(S, T, \mu)$ holds whenever $S$ and $T$ are products of intervals. By additivity, it thus holds whenever $S$ and $T$ are in $\mathcal{D}$, the set of finite unions of products of intervals. Since $d_{\mu_{n}}\left(\kappa_{1}, \kappa_{2}\right)=\sup _{S, T} f\left(S, T, \mu_{n}\right)$, for $S, T \in \mathcal{D}$ we thus have

$$
f(S, T, \mu)=\liminf f\left(S, T, \mu_{n}\right) \leq \liminf d_{\mu_{n}}\left(\kappa_{1}, \kappa_{2}\right)=d_{\text {cut }}\left(\kappa_{1}, \kappa_{2}\right) .
$$


As noted earlier, when defining $d_{\mu}\left(\kappa_{1}, \kappa_{2}\right)=\sup _{S, T} f(S, T, \mu)$, we may take the supremum instead over $S, T \in \mathcal{D}$, so it follows that $d_{\mu}\left(\kappa_{1}, \kappa_{2}\right) \leq d_{\text {cut }}\left(\kappa_{1}, \kappa_{2}\right)$. Since $d_{\text {cut }}\left(\kappa_{1}, \kappa_{2}\right)=\inf _{\mu \in \mathcal{M}} d_{\mu}\left(\kappa_{1}, \kappa_{2}\right)$, this infimum is attained, as claimed.

It remains to prove (13). But this is easy: for any interval $I \subset[0,1]$, let $\mu_{n}^{I}$ be the measure on $[0,1]$ defined by

$$
\mu_{n}^{I}(A)=\mu_{n}(A \times I),
$$

and define $\mu^{I}$ from $\mu$ similarly. Recall that $\mu_{n} \rightarrow \mu$ on products of intervals. Thus $\mu_{n}^{I}(A) \rightarrow \mu^{I}(A)$ whenever $A$ is an interval, and hence whenever $A$ is a finite union of intervals. Since $\mu_{n}, \mu \in \mathcal{M}$, we have $\mu_{n}(A), \mu(A) \leq|A|$ for any $A$, where $|\cdot|$ denotes Lebesgue measure. It follows that $\mu_{n}^{I}(A) \rightarrow \bar{\mu}^{I}(A)$ for any measurable $A \subset[0,1]$, since for any $\varepsilon$ we can approximate $A$ by a finite union of intervals $A^{\prime}$ with $\left|A \Delta A^{\prime}\right| \leq \varepsilon$. It also follows that if $I$ and $J$ are two intervals, and $A \subset[0,1]^{2}$ is Lebesgue measurable, then

$$
\left(\mu_{n}^{I} \times \mu_{n}^{J}\right)(A) \rightarrow\left(\mu^{I} \times \mu^{J}\right)(A) .
$$

(Simply approximate $A$ by a finite union of products of intervals.) Considering level sets, it follows immediately that

$$
\int f(x, y) d \mu_{n}^{I}(x) d \mu_{n}^{J}(y) \rightarrow \int f(x, y) d \mu^{I}(x) d \mu^{J}(y)
$$

for any bounded measurable function $f$. Taking $f=\kappa(x, y) 1_{x \in S_{1}} 1_{y \in T_{1}}, I=S_{2}$ and $J=T_{2}$, this is exactly (13), completing the proof.

The special case of Lemma 2.4 where the distance is 0 is of particular interest.

Corollary 2.5. Let $\kappa_{1}$ and $\kappa_{2}$ be two kernels. Then $d_{\text {cut }}\left(\kappa_{1}, \kappa_{2}\right)=0$ if and only if $\kappa_{1} \sim \kappa_{2}$.

Proof. Using (11) as the definition of $d_{\text {cut }}$, if $\kappa_{1} \sim \kappa_{2}$ then we certainly have $d_{\text {cut }}\left(\kappa_{1}, \kappa_{2}\right)=0$; see (9)).

Suppose then than $d_{\text {cut }}\left(\kappa_{1}, \kappa_{2}\right)=0$. From Lemma 2.4. there is a $\mu \in \mathcal{M}$ such that $d_{\mu}\left(\kappa_{1}, \kappa_{2}\right)=0$. Let $\nu$ be the signed measure on $[0,1]^{4}$ defined by

$$
d \nu(x, u, y, v)=\left(\kappa_{1}(x, y)-\kappa_{2}(u, v)\right) d \mu(x, u) d \mu(y, v) .
$$

Then $d_{\mu}\left(\kappa_{1}, \kappa_{2}\right)=0$ says exactly that $\nu(S \times T)=0$ for all measurable $S, T \subset$ $[0,1]^{2}$. Since $\nu$ is a signed Borel measure, it follows immediately that $\nu$ is the zero measure. Equivalently, $\kappa_{1}(x, y)-\kappa_{2}(u, v)=0$ for $(\mu \times \mu)$-a.e. points $(x, u, y, v)$. Referring to (9) again, we see that $\kappa_{1} \sim \kappa_{2}$.

Corollaries 2.3 and 2.5 together imply the following result.

Theorem 2.6. Let $\kappa_{1}$ and $\kappa_{2}$ be two kernels. Then $s\left(F, \kappa_{1}\right)=s\left(F, \kappa_{2}\right)$ holds for every finite graph $F$ if and only if $\kappa_{1} \sim \kappa_{2}$. 
Theorem 2.6 is a very basic fact about kernels: it says that a kernel is characterized up to equivalence by the quantities $s(F, \kappa)$, which are the natural analogues for a kernel of the moments of a random variable. One would hope that Theorem 2.6 would have a simple direct proof (as the corresponding statement for random variables does), but so far the only proof we know uses Theorem 2.2 i.e., it relies on the hard results of Borgs, Chayes, Lovász, Sós and Vesztergombi [17]. (In fact, as we shall see later, Theorem 2.2 is not that hard to deduce from Theorem 2.6.)

Borgs, Chayes, Lovász, Sós and Vesztergombi [15] announced an equivalence result closely related to Theorem 2.6. the proof of which is to appear in Borgs, Chayes and Lovász [14. Recall from (10) that $\kappa_{1} \sim \kappa_{2}$ means that

$$
\exists \kappa, \sigma_{1}, \sigma_{2} \text { such that } \kappa=\kappa_{1}^{\left(\sigma_{1}\right)} \text { a.e and } \kappa=\kappa_{2}^{\left(\sigma_{2}\right)} \text { a.e, }
$$

where $\kappa$ is a kernel on $[0,1]$ and $\sigma_{1}$ and $\sigma_{2}$ are measure-preserving maps from $[0,1]$ to itself. Turning this 'upside-down', let us write $\kappa_{1} \sim^{\prime} \kappa_{2}$ if

$$
\exists \kappa, \sigma_{1}, \sigma_{2} \text { such that } \kappa_{1}=\kappa^{\left(\sigma_{1}\right)} \text { a.e and } \kappa_{2}=\kappa^{\left(\sigma_{2}\right)} \text { a.e. }
$$

In (14), we require $\kappa$ to be a kernel on $[0,1]$; it makes no difference if we allow $\kappa$ to be a kernel on an arbitrary standard probability space. The uniqueness result of 14 announced in 15 concerns the relation $\sim^{\prime}$ rather than $\sim$ : it states that $s\left(F, \kappa_{1}\right)=s\left(F, \kappa_{2}\right)$ for every $F$ if and only if $\kappa_{1} \sim^{\prime} \kappa_{2}$. In the light of Theorem 2.6. this implies, rather indirectly, that $\kappa_{1} \sim^{\prime} \kappa_{2}$ if and only if $\kappa_{1} \sim \kappa_{2}$. Of course, one would like a direct proof of this statement.

Question 2.7. Is there a direct proof that, for two kernels $\kappa_{1}$ and $\kappa_{2}$ on $[0,1]$, we have $\kappa_{1} \sim^{\prime} \kappa_{2}$ if and only if $\kappa_{1} \sim \kappa_{2}$ ?

Note that one implication is trivial: if $\kappa_{1} \sim^{\prime} \kappa_{2}$, then using $\kappa \sim \kappa^{(\sigma)}$ twice, we have $\kappa_{1} \sim \kappa_{2}$. The reverse implication is very easy for finite-type kernels, but may involve measure-theoretic complications in the general case.

Our aim in this paper is to investigate the extent to which the various results and observations above carry over to sparse graphs, graphs with $n$ vertices and $o\left(n^{2}\right)$ edges. Unfortunately, this seems to give rise to many difficult questions, and we shall present many more questions than answers.

\section{Subgraph counts for sparse graphs}

In this section we consider sparse graphs, where the number of edges is $o\left(n^{2}\right)$ as the number $n$ of vertices goes to infinity. We shall assume throughout that we have at least $\omega(n)$ edges, i.e., that the average degree tends to infinity; often, we shall make much stronger assumptions. Given a function $p=p(n)$, one can adapt many of the notions of Section 2 to graphs with $\Theta\left(p n^{2}\right)$ edges. Indeed, let

$$
s_{p}(F, G)=\frac{\operatorname{emb}(F, G)}{p^{e(F)} n_{(|F|)}}=\operatorname{aut}(F) \frac{X_{F}(G)}{p^{e(F)} X_{F}\left(K_{n}\right)},
$$


noting that

$$
s_{p}(F, G)=\frac{\operatorname{emb}(F, G)}{\mathbb{E}(\operatorname{emb}(F, G(n, p)))}
$$

is the ratio of the number of copies of $F$ in $G$ to the expected number of copies in $G(n, p)$. Also, let

$$
t_{p}(F, G)=\frac{\operatorname{hom}(F, G)}{p^{e(F)} n^{|F|}} .
$$

If $p=1$, then we recover the definitions in Section 2. Furthermore, if $0<p<1$ is constant, then we can define a map $s$ as before, but now $s$ maps $\mathcal{F}$ into the compact space $\prod_{F \in \mathcal{F}}\left[0, p^{-e(F)}\right]$, and everything proceeds as before. More generally, changing $p$ by a constant factor will be irrelevant: just as we can use $s_{c}$ for any $c$ to study $G(n, 1 / 2)$, we may use $s_{p}$ to study $G(n, p / 2)$ or $G(n, 2 p)$, say, for any $p=p(n)$.

From now on, we suppose that $p=p(n)$ is some given function of $n$, with $p(n) \rightarrow 0$ as $n \rightarrow \infty$. We wish to work in a compact space, so we shall assume that there are constants $c_{F}, F \in \mathcal{F}$, such that $s_{p}(F, G) \leq c_{F}$ for all graphs $G$ we consider. Enumerating $\mathcal{F}$ as $\left\{F_{1}, F_{2}, \ldots\right\}$, we may thus define a map

$$
s_{p}: \mathcal{F} \rightarrow X=\prod_{i=1}^{\infty}\left[0, c_{F_{i}}\right], \quad G \mapsto\left(s_{p}\left(F_{i}, G\right)\right)_{i=1}^{\infty},
$$

and, using any metric $d$ on $X$ giving the product topology, an associated metric

$$
d_{\mathrm{sub}}\left(G_{1}, G_{2}\right)=d\left(s_{p}\left(G_{1}\right), s_{p}\left(G_{2}\right)\right)
$$

We suppress the dependence on $p$ in our notation for the metric to avoid clutter. As in the dense case, we can extend $d_{\text {sub }}$ to kernels $\kappa$, setting

$$
d_{\mathrm{sub}}(G, \kappa)=d\left(s_{p}(G), s(\kappa)\right) \quad \text { and } \quad d_{\mathrm{sub}}\left(s\left(\kappa_{1}\right), s\left(\kappa_{2}\right)\right)=d\left(s\left(\kappa_{1}\right), s\left(\kappa_{2}\right)\right)
$$

for a graph $G$ and kernels $\kappa, \kappa_{1}$ and $\kappa_{2}$. Here, for a kernel $\kappa, s(\kappa)$ is the vector with coordinates defined by (1).

Much of the time, we think of a sequence $\left(G_{n}\right)$ of finite graphs. Throughout, we are only interested in sequences with $\left|G_{n}\right| \rightarrow \infty$. For notational convenience we always assume that $\left|G_{n}\right|=n$; this make no difference to our conjectures and results. As usual, we need not assume that $G_{n}$ is defined for every $n \in \mathbb{N}$, but only for an infinite subset of $\mathbb{N}$. In this setting, the assumption described above may be stated as follows.

Assumption 3.1 (bounded subgraph counts). For each $F$, the parameter $s_{p}\left(F, G_{n}\right)$ is bounded.

In particular, if $\left(G_{n}\right)$ satisfies Assumption 3.1 then, taking $F=K_{2}$, we see that $e\left(G_{n}\right)=O\left(p n^{2}\right)$, so our graphs are sparse. There is a stronger version of Assumption 3.1 that is perhaps even more natural: 
Assumption 3.2 (exponentially bounded subgraph counts). There is a constant $C$ such that, for each fixed $F$, we have $\limsup s_{p}\left(F, G_{n}\right) \leq C^{e(F)}$ as $n \rightarrow \infty$.

In this case, changing $p$ by a constant factor, we may take $C=1$ if we like. This is not always the most natural normalization, however. There is a reason for writing limsup in Assumption 3.2 for any graph $G_{n}$ with $\left|G_{n}\right|=n$ and $n$ large, there will be some $F$ with $s_{p}\left(F, G_{n}\right)$ very large. Indeed, $G_{n}$ contains at least one embedding of itself, so $s_{p}\left(G_{n}, G_{n}\right) \geq 1 /\left(n ! p^{e\left(G_{n}\right)}\right)$, which typically grows much faster than any constant to the power $e\left(G_{n}\right)$.

Turning to kernels, there is no longer any good reason to restrict our kernels to take values in $[0,1]$ : in the dense case, the maximum possible 'local density' of edges is 1 . Here, if we normalize so that $G_{n}$ has $p n^{2}$ edges, say, local densities larger than $p$ are certainly possible. From now on, by a kernel we thus mean a symmetric measurable function $\kappa$ from $[0,1]^{2}$ to the non-negative reals. We define $s(F, \kappa)$ as before, using (11); in general, $s(F, \kappa)$ may be infinite, but we shall always assume it is finite for the graphs $F$ and kernels $\kappa$ we consider.

Although we allow unbounded kernels in general, it may be that they give rise to difficulties (as they do in the general (very) sparse inhomogeneous model of Bollobás, Janson and Riordan [10]). Assumption [3.2 corresponds to the limiting kernel (if it exists) being bounded, as shown by Lemma 3.5 below.

Our main conjecture states that, if $p$ is large enough, then, under Assumption 3.2. the equivalent of Theorem 2.1 holds.

Conjecture 3.3. Let $p=p(n)=n^{-o(1)}$, and let $C>0$ be constant. Suppose that $\left(G_{n}\right)$ is a sequence of graphs with $\left|G_{n}\right|=n$ such that, for every $F, s_{p}\left(F, G_{n}\right)$ converges to some constant $0 \leq c_{F} \leq C^{e(F)}$. Then there is a bounded kernel $\kappa$ such that $c_{F}=s(F, \kappa)$ for every $F$.

As noted above, without loss of generality we may take $C=1$. As we shall note later, it is very easy to see that if $s_{p}\left(K_{2}, G_{n}\right) \rightarrow 0$ and $s_{p}\left(F, G_{n}\right)$ is bounded for every $F$, then $s_{p}\left(F, G_{n}\right) \rightarrow 0$ for every $F$. Thus we may assume that $s_{p}\left(K_{2}, G_{n}\right)$ is bounded away from zero, and we may normalize in a different way by assuming that $s_{p}\left(K_{2}, G_{n}\right)=1$, i.e, that $e\left(G_{n}\right)=p\left(\begin{array}{l}n \\ 2\end{array}\right)$.

Assumption 3.2 is trivially stronger than Assumption 3.1 Thus, if $\left(G_{n}\right)$ satisfies Assumption 3.2 then the sequence $s_{p}\left(G_{n}\right)$ lives in a compact product space, and has a convergent subsequence. Hence, there are real numbers $c_{F} \geq 0$, $F \in \mathcal{F}$, and a subsequence $\left(G_{n_{i}}\right)$ with $s_{p}\left(F, G_{n_{i}}\right) \rightarrow c_{F}$ for every $F$ to which Conjecture 3.3 applies. Conjecture 3.3 is thus a statement about the possible limit points of the sequences $s_{p}\left(G_{n}\right)$.

It may well be that the restriction to bounded kernels is not necessary.

Conjecture 3.4. Let $p=p(n)=n^{-o(1)}$, and let $\left(G_{n}\right)$ be a sequence of graphs with $\left|G_{n}\right|=n$, such that for every $F$ we have $s_{p}\left(F, G_{n}\right) \rightarrow c_{F}$ for some $0 \leq$ $c_{F}<\infty$. Then there is a kernel $\kappa$ with $c_{F}=s(F, \kappa)$ for every $F$.

We have stated the above conjectures under the assumption that $p=n^{-o(1)}$; we shall call this the almost dense case. The reason for this assumption is 
discussed further below. Let us note that, in the almost dense case, for each fixed $F$ we have $t_{p}\left(F, G_{n}\right) \sim s_{p}\left(F, G_{n}\right)$ as $n \rightarrow \infty$, so it makes no difference whether we consider $s_{p}$ or $t_{p}$. In general, this is not true: for example, considering homomorphisms which map $V\left(K_{t, t}\right)$ into a set of $2 t-1$ vertices shows that with $F=K_{t, t}$ we need $n p^{t} \rightarrow \infty$ for $t_{p}\left(F, G_{n}\right) \sim s_{p}\left(F, G_{n}\right)$ to hold in general.

\subsection{Bounded and unbounded kernels}

The following simple observation illuminates the relationship between Conjectures 3.3 and 3.4

Lemma 3.5. Let $\kappa:[0,1]^{2} \rightarrow \mathbb{R}^{+}$be a kernel, and $C \geq 0$ a constant. Then we have $s(F, \kappa) \leq C^{e(F)}$ for every $F$ if and only if $\kappa \leq C$ holds almost everywhere.

Proof. The result is trivial if $C=0$. Otherwise, rescaling, we may assume that $C=1$. If $\kappa \leq 1$ almost everywhere, then $s(F, \kappa) \leq s(F, 1)=1$ for every $F$. We may thus suppose that $\kappa>1$ on a set of positive measure. It follows that there is some $\eta>0$ such that $\kappa>(1+\eta)^{2}$ on a set $A$ of positive measure. Applying the Lebesgue Density Theorem to $A$, there is some $\varepsilon>0$ and some rectangle $R=[a, a+\varepsilon] \times[b, b+\varepsilon] \subset[0,1]^{2}$ such that $\mu(A \cap R) \geq \mu(R) /(1+\eta)$. Thus, the average value of $A$ on the set $R$ is at least $1+\eta$. Let $\kappa^{\prime}$ be the kernel taking the value $1+\eta$ on $R$ and 0 elsewhere. Standard arguments from convexity show that, for each $t$,

$$
s\left(K_{t, t}, \kappa\right) \geq s\left(K_{t, t}, \kappa^{\prime}\right)=\varepsilon^{2 t}(1+\eta)^{t^{2}} .
$$

Taking $t$ large enough, we find an $F=K_{t, t}$ for which $s(F, \kappa)>1$.

Lemma 3.5 shows that a kernel $\kappa$ is bounded if and only if the counts $s(F, \kappa)$ grow at most exponentially in $e(F)$. It also shows that, in Conjecture 3.3, we need only consider kernels $\kappa:[0,1]^{2} \rightarrow[0, C]$.

Let us say that a kernel has finite moments if $s(F, \kappa)<\infty$ for all $F$. There are unbounded kernels with finite moments: the simplest way to construct such an example is to consider the 'rank 1' case, where $\kappa(x, y)=f(x) f(y)$ for some $f:[0,1] \rightarrow \mathbb{R}^{+}$. Indeed, let $f$ be any function from $[0,1]$ to $\mathbb{R}^{+}$with $\mathbb{E}\left(f^{k}\right)=$ $\int_{0}^{1} f(x)^{k} d x$ bounded for every $k$; for example, let $f(x)=\log (1 / x)$ for $x>0$. Set $\kappa(x, y)=f(x) f(y)$. If $F$ is a graph on $\{1,2, \ldots, k\}$ in which vertex $i$ has degree $d_{i}$, then

$$
\begin{aligned}
s(F, \kappa)=\int_{[0,1]^{k}} \prod_{i j \in E(F)} f\left(x_{i}\right) & f\left(x_{j}\right) \prod_{i=1}^{k} d x_{i} \\
= & \int_{[0,1]^{k}} \prod_{i=1}^{k} f\left(x_{i}\right)^{d_{i}} \prod_{i=1}^{k} d x_{i}=\prod_{i=1}^{k} \mathbb{E}\left(f^{d_{i}}\right)<\infty .
\end{aligned}
$$

The calculation above shows that a rank one kernel $\kappa(x, y)=f(x) f(y)$ has finite moments if and only if $\|f\|_{p}<\infty$ for every $p>1$, and hence if and only if 
$\|\kappa\|_{p}<\infty$ for every $p>1$. It is tempting to think that this holds in general. In one direction, for any kernel $\kappa$ and any graph $F$ on $\{1,2, \ldots, k\}$, we may write

$$
s(F, \kappa)=\int_{[0,1]^{k}} \prod_{i j \in E(F)} \kappa_{i j}\left(x_{1}, \ldots, x_{d}\right) \prod_{i=1}^{k} d x_{i},
$$

where $\kappa_{i j}\left(x_{1}, \ldots, x_{d}\right)=\kappa\left(x_{i}, x_{j}\right)$. Thus, by Hölder's inequality,

$$
s(F, \kappa)=\int \prod_{i j \in E(F)} \kappa_{i j} \leq \prod_{i j \in E(F)}\left\|\kappa_{i j}\right\|_{e(F)}=\prod_{i j \in E(F)}\|\kappa\|_{e(F)} .
$$

Hence, if $\|\kappa\|_{p}<\infty$ for every $p>1$, then $s(F, \kappa)<\infty$ for every $F$. The reverse implication does not hold, however, as shown by the following example.

Example 3.6. A kernel with finite moments but infinite 2-norm. Let us define a sequence of independent random kernels $\kappa_{0}, \kappa_{1}, \kappa_{2}, \ldots$, as follows. For $r \geq 0$, let $\mathcal{P}_{r}$ be the partition of $[0,1]$ into $2^{2^{r}}$ equal intervals, and let $\mathcal{P}_{r}^{2}$ be the corresponding partition of $[0,1]^{2}$ : divide $[0,1]^{2}$ into $4^{2^{r}}$ squares in the obvious way, and take as one part of $\mathcal{P}_{r}^{2}$ the union of a square and its reflection in the line $x=y$ (which may be the same square). Our kernel $\kappa_{r}$ will be constant on each element of $\mathcal{P}_{r}^{2}$, taking the value $2^{r}$ with probability $2^{-2 r}$ and 0 otherwise, with the values on different squares independent. Note that $\kappa_{0}$ is simply the constant kernel with value 1 .

Let $\kappa(x, y)=\sum_{r=0}^{\infty} \kappa_{r}(x, y)$. It is easy to see that with probability 1 the sum converges almost everywhere (for example, using the fact that $\mathbb{E} \mu\left\{\kappa_{r}>\right.$ $0\}=2^{-2 r}$ to deduce that, with probability $1, \mu\left\{\exists s>r: \kappa_{s}>0\right\}$ tends to 0 as $r \rightarrow \infty$. Also, for large $r,\left\|\kappa_{r}\right\|_{2}^{2}$ is concentrated around its mean of $\left(2^{r}\right)^{2} 2^{-2 r}=1$. Hence, with probability 1 we have $\left\|\kappa_{r}\right\|_{2}^{2} \geq 0.99$ for infinitely many $r$. Using $(a+b)^{2} \geq a^{2}+b^{2}$ for $a, b \geq 0$, it follows that $\|\kappa\|_{2}^{2}$ is infinite with probability 1 ; in particular, $\kappa$ does not have all $p$-norms finite.

Turning to the finite moments property, let $F$ be any fixed graph, with $t$ vertices. Since $\kappa \geq \kappa_{0}=1$, we have $s(F, \kappa) \leq s\left(K_{t}, \kappa\right)$, so we may assume without loss of generality that $F=K_{t}$. Since $\kappa$ is random, $s\left(K_{t}, \kappa\right)$ is a random variable. We may write its expectation as

$$
\mathbb{E}_{\kappa} \mathbb{E}_{\mathbf{x}} \prod_{i<j} \kappa\left(x_{i}, x_{j}\right)=\mathbb{E}_{\mathbf{x}} \mathbb{E}_{\kappa} \prod_{i<j} \kappa\left(x_{i}, x_{j}\right)
$$

where $\mathbb{E}_{\kappa}$ denotes expectation over the random choice of $\kappa$, and $\mathbb{E}_{\mathbf{x}}$ over the random choice of $\left(x_{1}, \ldots, x_{t}\right)$, a sequence of $t$ iid uniform elements of $[0,1]$. Let us fix $\mathbf{x}$ for the moment, assuming as we may that $x_{i} \neq x_{j}$ for $i \neq j$. Let $\ell$ be the largest $r$ such that some pair $x_{i}, x_{j}$ lie in the same part of $\mathcal{P}_{r}$, so $0 \leq \ell<\infty$. Let $\sigma=\sum_{r \leq \ell} \kappa_{r}$ and $\tau=\sum_{r>\ell} \kappa_{r}$, so $\kappa=\sigma+\tau$. For $r>\ell$, the $\left(\begin{array}{l}t \\ 2\end{array}\right)$ pairs $\left(x_{i}, x_{j}\right), i<\bar{j}$, all lie in different parts of $\mathcal{P}_{r}^{2}$, so the values of $\kappa_{r}$ on these pairs are independent. Since different $\kappa_{r}$ are independent, it follows that the values 
of $\tau$ on the pairs are also independent. Now $\|\sigma\|_{\infty} \leq \sum_{r=0}^{\ell}\left\|\kappa_{r}\right\|_{\infty}=2^{\ell+1}-1$. Thus,

$$
\mathbb{E}_{\kappa} \prod_{i<j} \kappa\left(x_{i}, x_{j}\right) \leq \mathbb{E}_{\kappa} \prod_{i<j}\left(2^{\ell+1}+\tau\left(x_{i}, x_{j}\right)\right)=\prod_{i<j}\left(2^{\ell+1}+\mathbb{E}_{\kappa} \tau\left(x_{i}, x_{j}\right)\right) .
$$

For any $x$ and $y$ we have $\mathbb{E}_{\kappa} \kappa_{r}(x, y)=2^{r} 2^{-2 r}=2^{-r}$, from which it follows that $\mathbb{E}_{\kappa} \tau(x, y) \leq 2$, and hence, very crudely, that

$$
\mathbb{E}_{\kappa} \prod_{i<j} \kappa\left(x_{i}, x_{j}\right) \leq \prod_{i<j}\left(2^{\ell+1}+2\right) \leq 2^{2 \ell t^{2}} .
$$

It remains to take the expectation over $\mathbf{x}$. Since $\mathbb{P}(\ell=r) \leq\left(\begin{array}{c}t \\ 2\end{array}\right) 2^{-2^{r}}$, we find that

$$
\mathbb{E} s(F, \kappa) \leq \sum_{r=0}^{\infty}\left(\begin{array}{l}
t \\
2
\end{array}\right) 2^{-2^{r}} 2^{2 r t^{2}}<\infty,
$$

noting that for any fixed $t$ the $2^{-2^{r}}$ term dominates. If follows that with probability 1 we have $s(F, \kappa)<\infty$ for every $F$, giving a kernel with finite moments but with $\|\kappa\|_{2}$ infinite. A simple modification, taking the probability that $\kappa_{r}$ takes the value $2^{r}$ on a given square to be $2^{-(1+\varepsilon) r}$ rather than $2^{-2 r}$ gives, for each $\varepsilon>0$, an example with $\|\kappa\|_{1+\varepsilon}$ infinite.

\subsection{Non-uniform random graphs}

As in the dense case, there is a key connection between convergence of the counts $s_{p}\left(F, G_{n}\right)$ and random graphs. Given a kernel $\kappa$, let $G_{p}(n, \kappa)$ be the random graph $G(n, p \kappa)$. More precisely, let $G_{p}(n, \kappa)$ be the random graph on $[n]$ obtained as follows: first choose $x_{1}, \ldots, x_{n}$ independently and uniformly from $[0,1]$. Then, conditional on this choice, join each pair $\{i, j\}$ of vertices independently, with probability $\min \left\{p \kappa\left(x_{i}, x_{j}\right), 1\right\}$. We write the parameter $p$ as a subscript to emphasize that it is part of the overall normalization: we think of a sparse graph generated from the kernel $\kappa$, rather than a 'sparse kernel' $p \kappa$. If $p=1 / n$, then $G_{p}(n, \kappa)$ is a special case of the general sparse inhomogeneous model of Bollobás, Janson and Riordan [10].

Remark 3.7. In what follows, we shall consider many statements about the convergence of various sequences of random graphs. As usual in the theory of random graphs, the precise notion of convergence is not important: one thinks of 'a random graph' with certain asymptotic properties, although this makes no formal sense. Formally, it is most natural to work throughout with convergence in probability, but this would require us to consider 'in probability' versions of our various assumptions, for example the (exponentially) bounded counts Assumptions 3.1 and 3.2. In fact, it is easy to check that in all cases considered here, the error probabilities decay fast enough to give almost sure convergence. However, we shall not verify this explicitly, noting that one can in any case ensure almost sure convergence by passing to a suitable subsequence. 
Lemma 3.8. Let $p=p(n)=n^{-o(1)}$, and let $\kappa$ be a kernel with $s(F, \kappa)<$ $\infty$ for every $F$. Then $s_{p}\left(F, G_{p}(n, \kappa)\right) \stackrel{\mathrm{p}}{\rightarrow} s(F, \kappa)$ for each fixed graph $F$, so $d_{\mathrm{sub}}\left(G_{p}(n, \kappa), \kappa\right) \stackrel{\mathrm{p}}{\rightarrow} 0$. In fact, the sequence $G_{p}(n, \kappa)$ converges almost surely to $\kappa$ in the metric $d_{\text {sub }}$.

Proof. It very easy to check that, for every $F, s_{p}\left(F, G_{p}(n, \kappa)\right)$ is concentrated around its mean $s(F, \kappa)$ : indeed, the second moment of the number of copies of $F$ can be written as a sum of terms $(1+o(1)) n_{(|H|)} p^{e(H)} s_{p}(H, \kappa)$, and the dominant term is the unique one with the largest power of $n$, where $H$ is the disjoint union of copies of $F$. (The $1+o(1)$ correction is only needed if $\kappa$ is unbounded, and appears due to the $\max \{1, \cdot\}$ in the edge probabilities.) This proves the first part of the result. Convergence in probability in $d_{\text {sub }}$ follows since convergence in probability in a product topology is equivalent to convergence in probability of each coordinate. For the final statement, see Remark 3.7

Lemma 3.8 implies that the sequence $G_{n}=G_{p}(n, \kappa)$ has bounded subgraph counts (i.e., satisfies Assumption 3.1) with probability 1. If $\kappa$ is bounded, then $G_{n}$ has exponentially bounded subgraph counts with probability 1.

Using Lemma 3.8 it is easy to see that we must allow unbounded kernels in Conjecture 3.4. Indeed, set $\kappa(x, y)=\log (1 / x) \log (1 / y)$ for $0<x, y \leq 1$, say, and let $p(n)=1 / \log n$. Then the random graphs $G_{p}(n, \kappa)$ satisfy Assumption 3.1 with probability 1 , and

$$
s_{p}\left(F, G_{p}(n, \kappa)\right) \rightarrow s(F, \kappa)<\infty
$$

holds with probability 1 for every $F$. Since $\kappa$ is unbounded, by Lemma 3.5 there is no $C$ with $s(F, \kappa) \leq C^{e(F)}$ for every $F$, so there is no bounded $\kappa^{\prime}$ with $s_{p}\left(F, G_{p}(n, \kappa)\right) \rightarrow s\left(F, \kappa^{\prime}\right)$ for every $F$.

Note that if $p$ decreases too fast with $n$, then $s_{p}\left(F, G_{p}(n, \kappa)\right)$ is no longer concentrated around its mean: for example, this is the case if the mean does not tend to infinity. This is the reason for the assumption $p=n^{-o(1)}$ in the various conjectures and results above: otherwise, there will be some $F$ for which the mean does not tend to infinity. Note also that, for smaller $p$, when $s_{p}(F, \cdot)$ and $t_{p}(F, \cdot)$ are no longer asymptotically equal, the former is the more natural parameter: for a given $F$, the lower limit on $p$ below which the corresponding parameter for $G_{p}(n, \kappa)$ is no longer close to $s(F, \kappa)$ is in general much smaller for $s_{p}(F, \cdot)$ than for $t_{p}(F, \cdot)$. It may well be, however, that the conjectures in this section (or perhaps just their proofs) fail when the relevant parameters $s_{p}(F, \cdot)$ and $t_{p}(F, \cdot)$ are no longer asymptotically equal.

\subsection{Subgraph counts in the uniform case}

Using convexity, it is very easy to check that the only possible kernel $\kappa$ with $s\left(K_{2}, \kappa\right)=s\left(C_{4}, \kappa\right)=1$ is the uniform kernel, with $\kappa=1$ a.e. The following conjecture is thus a very special case of Conjecture 3.4. 
Conjecture 3.9. Let $p=p(n)=n^{-o(1)}$, and let $\left(G_{n}\right)$ be a sequence of graphs with $\left|G_{n}\right|=n, e\left(G_{n}\right)=p\left(\begin{array}{c}n \\ 2\end{array}\right), s_{p}\left(C_{4}, G_{n}\right) \rightarrow 1$, and $\sup _{n} s_{p}\left(F, G_{n}\right)<\infty$ for each $F$. Then $s_{p}\left(F, G_{n}\right) \rightarrow 1$ for every $F$.

Of course, there is a variant of Conjecture 3.9 where we replace Assumption 3.1 by Assumption 3.2, i.e., we demand that $\lim \sup _{n} s_{p}\left(F, G_{n}\right) \leq C^{e(F)}$ for some $C<\infty$. In this uniform context there is perhaps less reason to expect this to make a difference.

In the dense case, it is one of the basic results about quasi-random graphs that $s_{p}\left(K_{2}, G_{n}\right) \rightarrow 1$ and $s_{p}\left(C_{4}, G_{n}\right) \rightarrow 1$ imply $s_{p}\left(F, G_{n}\right) \rightarrow 1$ for every $F$, with no further assumptions. In the sparse case, this result extends easily to certain graphs $F$.

Lemma 3.10. Let $p=p(n)$ with $p / n^{-1 / 2} \rightarrow \infty$, and let $\left(G_{n}\right)$ be a sequence of graphs with $\left|G_{n}\right|=n$ such that $s_{p}\left(K_{2}, G_{n}\right) \rightarrow 1$ and $s_{p}\left(C_{4}, G_{n}\right) \rightarrow 1$. Then $s_{p}\left(C_{k}, G_{n}\right) \rightarrow 1$ for each $k \geq 5$.

Proof. Suppressing the dependence on $n$, let $A$ denote the adjacency matrix of $G_{n}$, and let $\lambda_{1} \geq \lambda_{2} \geq \cdots \geq \lambda_{n}$ be the eigenvalues of $A$. For $k \geq 3$ we have

$$
\operatorname{hom}\left(C_{k}, G_{n}\right)=\sum_{v_{1}, v_{2}, \ldots, v_{k} \in V\left(G_{n}\right)} A_{v_{1} v_{2}} A_{v_{2} v_{3}} \cdots A_{v_{k} v_{1}}=\operatorname{tr}\left(A^{k}\right)=\sum_{i=1}^{n} \lambda_{i}^{k} .
$$

It is easy to check that for $p$ in the given range we have $s_{p}\left(F, G_{n}\right) \sim t_{p}\left(F, G_{n}\right)$ whenever $F$ is a cycle. Thus, for each $k$, we have

$$
t_{p}\left(C_{k}, G_{n}\right) \sim s_{p}\left(C_{k}, G_{n}\right) \sim n^{-k} p^{-k} \sum_{i=1}^{n} \lambda_{i}^{k}=\sum_{i=1}^{n} \mu_{i}^{k}
$$

as $n \rightarrow \infty$, where $\mu_{i}=\lambda_{i} /(n p)$ is the $i$ th normalized eigenvalues of $G_{n}$. In particular,

$$
\sum_{i} \mu_{i}^{4} \rightarrow 1
$$

The maximum eigenvalue of the adjacency matrix of any graph is at least the average degree, so

$$
\mu_{1}=(n p)^{-1} \lambda_{1} \geq(n p)^{-1}(1+o(1))\left(n^{2} p\right) / n=1+o(1) .
$$

From (18) it follows that $\mu_{1} \sim 1$ and that $\sum_{i \geq 2} \mu_{i}^{4} \rightarrow 0$. Hence $\mu_{2} \leq 1$ and $\mu_{n} \geq-1$ if $n$ is large enough, and then

$\sum \mu_{i}^{k}=\mu_{1}^{k}+\sum_{i \geq 2} \mu_{i}^{k} \leq \mu_{1}^{k}+\max \left\{\mu_{2}^{k-4}, \mu_{n}^{k-4}\right\} \sum_{i \geq 2} \mu_{i}^{4} \leq \mu_{1}^{k}+\sum_{i \geq 2} \mu_{i}^{4}=1+o(1)$.

Using (17) again, the result follows. 
If instead of $s_{p}$ we work with $t_{p}$, then the proof above goes through without any restriction on $p$, so we appear to obtain a stronger result. In fact, this is only an illusion. Indeed, if $G_{n}$ has average degree $\bar{d}$, then it contains at least $n\left(\begin{array}{l}\bar{d} \\ 2\end{array}\right)$ pairs of adjacent edges. Thus, writing $N_{i, j}$ for the number of common neighbours of $i$ and $j$, the sum of $N_{i, j}$ over ordered pairs $i \neq j$ is at least $2 n\left(\begin{array}{l}\bar{d} \\ 2\end{array}\right)=n \bar{d}(\bar{d}-1)$. Hence, the number of homomorphisms from $C_{4}$ to $G_{n}$ with a given pair of opposite vertices mapped to distinct vertices is

$$
\sum_{i \neq j} N_{i, j}^{2} \geq \frac{1}{n(n-1)}\left(\sum N_{i, j}\right)^{2} \geq \frac{n \bar{d}^{2}(\bar{d}-1)^{2}}{n-1} .
$$

The number of homomorphisms with a given pair of opposite vertices mapped to the same vertex is simply the sum of the squares of the degrees in $G_{n}$, which is at least $n \bar{d}^{2}$. Thus,

$$
\operatorname{hom}\left(C_{4}, G_{n}\right) \geq \frac{n \bar{d}^{2}(\bar{d}-1)^{2}}{n-1}+n \bar{d}^{2}
$$

for any graph $G_{n}$ with $n$ vertices and average degree $\bar{d}$. With $\bar{d} \sim p n \rightarrow \infty$, this gives $\operatorname{hom}\left(C_{4}, G_{n}\right) \geq(1+o(1))\left(n^{4} p^{4}+n^{3} p^{2}\right)$, i.e., $t_{p}\left(C_{4}, G_{n}\right) \geq(1+o(1))(1+$ $\left.n^{-1} p^{-2}\right)$. Consequently, $t_{p}\left(C_{4}, G_{n}\right) \sim 1$ implies $p / n^{-1 / 2} \rightarrow \infty$, so under this assumption we are back in the range where $s_{p}\left(C_{4}, G_{n}\right) \sim t_{p}\left(C_{4}, G_{n}\right)$, and we may as well work with $s_{p}$. When $p / n^{-1 / 2} \rightarrow \infty$, (19) reduces to the well-known fact that, in this case, $e\left(G_{n}\right) \sim p\left(\begin{array}{l}n \\ 2\end{array}\right)$ implies that

$$
t_{p}\left(C_{4}, G_{n}\right), s_{p}\left(C_{4}, G_{n}\right) \geq 1-o(1) .
$$

In the dense case, Lemma 3.10 extends to triangles. Indeed, $\operatorname{tr}\left(A^{2}\right)$ counts the number of walks of length 2 in $G$, which is just $2 e(G)$. Thus

$$
\sum \mu_{i}^{2}=\frac{2 e(G)}{n^{2} p^{2}} \sim p^{-1}
$$

If $p$ is bounded away from zero then it follows that $\sum_{i \geq 2} \mu_{i}^{2}$ is bounded as $n \rightarrow$ $\infty$. Using (18) and the Cauchy-Schwartz inequality, it follows that $\sum_{i \geq 2} \mu_{i}^{3} \rightarrow$ 0 , and hence that $s_{p}\left(C_{3}, G_{n}\right) \rightarrow 1$.

If $p \rightarrow 0$, then this relation no longer holds. We start by considering the very sparse case, with $p \sim \sqrt{\log n} / \sqrt{n}$.

Example 3.11. Very sparse graphs with too few triangles. Let $p_{1}(n)$ and $p_{2}(n)$ be functions of $n$ satisfying $p_{2}=\left(1-p_{1}^{2}\right)^{n-2}$ and $p_{1}, p_{2}=\Theta(\sqrt{\log n} / \sqrt{n})$; for example, we may take $p_{2}=\sqrt{\log n} / \sqrt{n}$, in which case $p_{1} \sim p_{2} / \sqrt{2}$. Suppressing the dependence on $n$, let $G$ be the usual Erdős-Rényi random graph $G=G\left(n, p_{1}\right)$, and let $H$ be the graph on the same vertex set $[n]$ in which vertices $i$ and $j$ are joined if and only if they do not have a common neighbour in $G$. By our choice of $p_{1}$ and $p_{2}$, each edge of $H$ is present with probability $p_{1}$. For any set $E$ of $r=O(1)$ possible edges of $H$, the edges of $E$ are all present if 
and only if no vertex of $G$ is joined to both ends of some edge in $E$. Considering each vertex of $G$ separately, we see that the probability of this event is

$$
\left(1-r p_{1}^{2}+O\left(p_{1}^{3}\right)\right)^{n+O(1)}=e^{-r p_{1}^{2} n+O\left(n p_{1}^{3}\right)} \sim\left(\left(1-p_{1}^{2}\right)^{n-2}\right)^{r}=p_{2}^{r},
$$

where the $O(1)$ correction in the first exponent is to account for vertices that are endpoints of one or more edges in $E$. In other words, the probability that a bounded number of edges is present in $H$ is asymptotically the corresponding probability for $G\left(n, p_{2}\right)$.

For $E_{1}, E_{2} \subset E\left(K_{n}\right)$, the event $E_{2} \subset E(H)$ is a down-set in terms of $G$ (it says that certain pairs of edges of $G$ are not present), so $E_{2} \subset E(H)$ and $E_{1} \subset E(G)$ are negatively correlated. Hence, if $\left|E_{2}\right|=O(1)$, we have

$$
\mathbb{P}\left(E_{1} \subset E(G) \cap E_{2} \subset E(H)\right) \leq(1+o(1)) p_{1}^{\left|E_{1}\right|} p_{2}^{\left|E_{2}\right|} .
$$

Considering all ways of splitting a set $E$, it follows that $\mathbb{P}(E \subset G \cup H) \leq$ $(1+o(1))\left(p_{1}+p_{2}\right)^{|E|}$, and hence that

$$
\mathbb{E}\left(s_{p}(F, G \cup H)\right) \leq 1+o(1)
$$

for any fixed graph $F$, where $p=p_{1}+p_{2}$.

Since $G$ and $H$ overlap in very few edges, and the numbers of edges of $G$ and of $H$ are concentrated, we have $s_{p}\left(K_{2}, G \cup H\right) \rightarrow 1$ almost surely. It follows that $s_{p}\left(C_{4}, G \cup H\right) \geq 1-o(1)$ almost surely: we are in the range of $p$ where $t_{p}\left(C_{4}, \cdot\right) \sim s_{p}\left(C_{4}, \cdot\right)$. Hence, hence, from (21),

$$
s_{p}\left(C_{4}, G \cup H\right), t_{p}\left(C_{4}, G \cup H\right) \stackrel{\mathrm{p}}{\rightarrow} 1 .
$$

On the other hand, there are by definition no triangles with two edges in $G$ and one in $H$. Hence, from (20), the expectation of $\operatorname{emb}\left(K_{3}, G \cup H\right)$ is at most

$$
(1+o(1)) n^{3}\left(p_{1}^{3}+0+3 p_{1} p_{2}^{2}+p_{2}^{3}\right),
$$

so $\mathbb{E}\left(s_{p}\left(K_{3}, G \cup H\right)\right) \leq\left(p^{3}-3 p_{1}^{2} p_{2}\right) / p^{3}+o(1)$. Since $p_{1}, p_{2}$ and $p$ are all of the same order, this final fraction is strictly less than 1 , and our construction gives almost surely a sequence $G_{n}=G \cup H$ with $s_{p}\left(K_{2}, G_{n}\right) \rightarrow 1, s_{p}\left(C_{4}, G_{n}\right) \rightarrow 1$ but $s_{p}\left(C_{3}, G_{n}\right) \not \rightarrow 1$. Taking $p_{2} \sim p_{1} / 2$, we may achieve $s_{p}\left(C_{3}, G_{n}\right) \rightarrow 5 / 9$. Alternatively, choosing $p_{1}$ and $p_{2}$ suitably, we may find a sequence with $s_{p}\left(C_{3}, G_{n}\right)$ † 1 for any $p=p(n)$ satisfying $p / n^{-1 / 2} \rightarrow \infty$ and $p=O(\sqrt{\log n} / \sqrt{n})$.

Example 3.12. Denser graphs with too few triangles. Let $n=m k$ where $m \rightarrow \infty$, and let $p=\sqrt{\log m} / \sqrt{m}$. Example 3.11 gives us a graph $G^{\prime}$ of order $m$ with $t_{p}\left(K_{2}, G^{\prime}\right), t_{p}\left(C_{4}, G^{\prime}\right) \sim 1$ and $t_{p}\left(K_{3}, G^{\prime}\right) \leq 0.9$, say, for all large enough $m$. Let $G$ be the blow-up of $G^{\prime}$ obtained by replacing each vertex by $k$ vertices. Since $t_{p}(F, \cdot)$ is unchanged by blow-ups, we have $t_{p}\left(K_{2}, G\right), t_{p}\left(C_{4}, G\right) \sim$ 1 but $t_{p}\left(K_{3}, G\right) \leq 0.9$, from which $s_{p}\left(K_{2}, G\right), s_{p}\left(C_{4}, G\right) \sim 1$ and (for $n$ large) $s_{p}\left(K_{3}, G\right) \leq 0.91$ follow immediately. 
Although $p$ has not changed, the number of vertices has. Seen as a function of $n$, we may choose $p=\sqrt{\log m} / \sqrt{m}$ for any $m$ dividing $n$ with $m \rightarrow \infty$. Exact divisibility is not essential. Either by using this fact, or by restricting to a subsequence, we see that any given function $p(n)$ can be realized up to a factor of $(1+o(1))$, provided $p(n) /(\sqrt{\log n} / \sqrt{n}) \rightarrow \infty$ and $p(n)=o(1)$. Hence, we may construct graphs with the right number of $C_{4} \mathrm{~s}$ but too few triangles for any such function $p(n)$.

At first sight Example 3.12 seems to contradict Conjecture 3.9. but this is not the case. Indeed, for the graph $G^{\prime}$ that we blow up, (21) tells us that we do not have too many embeddings of any fixed $F$. However, while $s_{p} \sim t_{p}$ for $p=n^{o(1)}$, the final $p$ we consider, and while blowing up preserves $t_{p}, G^{\prime}$ is a very sparse graph: although it has the same absolute density as the final graph $G$, this density is much smaller than $\left|G^{\prime}\right|^{-o(1)}$, since $G^{\prime}$ has many fewer vertices than $G$. It follows that the homomorphism counts in $G^{\prime}$ are not well behaved. In particular, $G^{\prime}$ contains around $m^{4} p^{3}$ non-injective homomorphisms from $K_{2,3}$, which turns out to be much larger than the number $m^{5} p^{6}$ of embeddings. It follows that $G$ contains too many homomorphisms from, and thus embeddings of, $K_{2,3}$, i.e., that $s_{p}\left(K_{2,3}, G\right) \rightarrow \infty$.

One possible approach to producing a counterexample to Conjecture 3.9 would be to consider circulant graphs, i.e., graphs on the vertex set $[n]$ in which whether or not $i j$ is an edge depends only on $i-j$ modulo n. There is one circulant graph for each subset $A$ of the integers modulo $n$ satisfying $0 \notin A$ and $a \in A$ if and only if $-a \in A$. All our conjectures thus imply corresponding conjectures for subsets of $\mathbb{Z}_{n}$, the integers modulo $n$, in which the symmetry condition is not likely to be relevant. Most subgraph counts in the graph have a rather unnatural interpretation in terms of the corresponding sets; the exception is cycles, where the number of $k$-cycles in $G$ corresponds to ( $n$ times the) number of $k$-tuples in $A^{k}$ summing to 0 . There is a result corresponding to Lemma 3.10 for subsets of $\mathbb{Z}_{n}$, proved in the same way but using Fourier coefficients instead of eigenvalues. Unfortunately, Examples 3.11 and 3.12 also carry over to the set context, in a fairly straightforward way: instead of blowing up the graph, we replace each element of $A$ by a block of consecutive integers. This shows that any result of the kind we want about subsets of $\mathbb{Z}_{n}$ must involve conditions other than constraints on the number of tuples summing to 0 .

In the sparse case, even assuming that $p=n^{-o(1)}$, it is not true that $s_{p}\left(K_{2}, G_{n}\right) \rightarrow 1$ and $s_{p}\left(C_{4}, G_{n}\right) \rightarrow 1$ together imply $s_{p}\left(F, G_{n}\right) \rightarrow 1$ for every $F$. We have just seen one example, with $F=C_{3}$. There are also much simpler examples.

Example 3.13. Adding a dense part. Let $p=1 / \log n$, say, and let $m=$ $m(n)=n /(\log n)^{c}$ where $c>0$ is constant. (We ignore rounding to integers.) Let $G^{\prime}$ be any graph on $n-m$ vertices, and let $G$ be the disjoint union of $G^{\prime}$ and a complete graph on $m$ vertices. Since $K_{m}$ contains roughly $m^{|F|}$ embeddings of any fixed $F$, we have

$$
s_{p}(F, G) \sim s_{p}\left(F, G^{\prime}\right)+\frac{m^{|F|}}{p^{e(F)} n^{|F|}}=s_{p}\left(F, G^{\prime}\right)+(\log n)^{e(F)-c|F|} .
$$


Taking $G^{\prime}=G(n-m, p)$ and $c=3 / 2$, say, we have $s_{p}\left(K_{2}, G\right) \sim s_{p}\left(K_{2}, G^{\prime}\right) \sim 1$, $s_{p}\left(C_{4}, G\right) \sim s_{p}\left(C_{4}, G^{\prime}\right) \sim 1$, but $s_{p}\left(K_{4}, G\right) \sim 1+1=2$. Note that $s_{p}\left(K_{5}, G\right) \rightarrow$ $\infty$, so the assumptions of Conjecture 3.9 are not satisfied.

The above example is rather artificial: there are too many copies of $K_{4}$ (and of $K_{5}$ ), but these sit on a small number of vertices. However, the same effect can be achieved by taking the union on the same vertex set of $G(n, p)$ and a disjoint union of $n / m$ copies of $K_{m}$. Also, we can use complete bipartite graphs instead of complete graphs.

Example 3.14. A blown-up random graph. Let $n=m k$, where $k=k(n)$ and $m=m(n)$ both tend to infinity. (As usual, we ignore divisibility issues, or consider a sequence $n_{i} \rightarrow \infty$.) Let $G_{1}$ be the random graph $G(m, p)$, where $p=p(n)$, and let $G=G_{1}^{(k)}$ be formed by replacing each vertex of $G$ by an independent set of size $k$, and each edge by a $k$-by- $k$ complete bipartite graph. The number of edges of $G$ is $k^{2} e\left(G_{1}\right)$, which is asymptotically $k^{2} m^{2} p / 2=n^{2} p / 2$, so $s_{p}\left(K_{2}, G\right) \rightarrow 1$ in probability and almost surely. Similarly, for any fixed graph $F$, each embedding of $F$ into $G_{1}$ gives rise to $k^{|F|}$ embeddings into $G$; the expected number of embeddings arising in this way is essentially the expected number in $G(n, p)$, so asymptotically such embeddings contribute $1+o(1)$ to $s_{p}(F, G)$.

There are other embeddings of $F$ into $G$, however, where some distinct vertices of $F$ are mapped to the same vertex in $G_{1}$. For $C_{4}$, we have roughly $m^{2} p k^{4}$ such embeddings within our complete bipartite graphs, and roughly $2 m^{3} p^{2} k^{4}$ from embeddings involving three vertices of $G_{1}$. Provided $m p^{2} \rightarrow \infty$, we still have $s_{p}\left(C_{4}, G\right) \rightarrow 1$.

Suppose now that $m p^{t} \rightarrow c$, for some integer $t \geq 3$ and constant $0<c<\infty$; for example, set $p=1 / \log n, k=(\log n)^{t} / c$ and $m=c n /(\log n)^{t}$. Then we have roughly $m^{2+t} k^{2+t} p^{2 t}$ embeddings of $K_{2, t}$ into $G$ coming from embeddings into $G_{1}$. But we also have roughly $m^{1+t} k^{2+t} p^{t}$ embeddings into $G$ coming from maps from $K_{2, t}$ into $G_{1}$ sending the two vertices on one side to the same vertex. It is easy to check that these two are the dominant terms (mapping the two vertices on one side to the same place we gain $t$ factors of $1 / p$ and lose one factor of $m$; any other identifications gain fewer factors of $1 / p$ per factor of $m$ ), and it follows that $s_{p}\left(K_{2, t}, G\right) \rightarrow 1+1 / c$.

Taking a 'typical' sequence of random graphs constructed as above gives an example with $s_{p}\left(K_{2}, G_{n}\right) \rightarrow 1, s_{p}\left(C_{4}, G_{n}\right) \rightarrow 1$ (and indeed $s_{p}\left(K_{2, t^{\prime}}, G_{n}\right) \rightarrow 1$ for $\left.2 \leq t^{\prime}<t\right)$, but $s_{p}\left(K_{2, t}, G_{n}\right) \rightarrow 1+1 / c \neq 1$. Once again, the assumptions of Conjecture 3.9 are not satisfied, this time because $s_{p}\left(K_{2, t+1}, G_{n}\right) \rightarrow \infty$.

We have seen from the examples above, that if $p(n) \rightarrow 0$, then $s_{p}\left(K_{2}, G_{n}\right)$ and $s_{p}\left(C_{4}, G_{n}\right) \rightarrow 1$ do not themselves imply that $s_{p}\left(F, G_{n}\right) \rightarrow 1$ for every $F$. However, attempted counterexamples to Conjecture 3.9 seem to be doomed to failure by the the additional assumption that $s_{p}\left(F, G_{n}\right)$ is bounded for every $F$. In the next section we shall see that we can make some progress towards proving Conjecture 3.9. 


\subsection{Partial results in the uniform case}

In the examples in the previous subsection, each vertex is in about the same number of copies of any fixed graph $F$, but there are relatively few $\left(o\left(n^{2}\right)\right)$ pairs that are in too many copies of $K_{2, t}$, for example. It is easy to see that, under the assumptions of Conjecture 3.9. this cannot happen. In fact, we can make a much more general statement. For this it is convenient to work with homomorphism counts and $t_{p}\left(F, G_{n}\right)$ rather than $s_{p}\left(F, G_{n}\right)$. As noted above, this makes no real difference; it just removes some smaller order error terms from the formulae involved.

Let $F$ be a fixed graph, and $F^{\prime}$ a subgraph of $F$. Without loss of generality, suppose that $V\left(F^{\prime}\right)=[\ell] \subset[k]=V(F)$. Then any homomorphism $\phi_{F}: F \rightarrow G_{n}$ restricts to a homomorphism $\phi_{F^{\prime}}: F^{\prime} \rightarrow G_{n}$. With $e\left(G_{n}\right) \sim p\left(\begin{array}{c}n \\ 2\end{array}\right)$, we expect a typical $\phi_{F^{\prime}}$ to have around $n^{k-\ell} p^{e(F)-e\left(F^{\prime}\right)}$ extensions. For each $n$, let us define a random variable $Z_{n}\left(F^{\prime}, F\right)$ as follows: let $\phi_{F^{\prime}}$ be chosen uniformly at random from among all homomorphisms from $F^{\prime}$ into $G$ (if there are any), and let $Z_{n}\left(F^{\prime}, F\right)$ be the number of extensions of $\phi_{F^{\prime}}$ divided by $n^{k-\ell} p^{e(F)-e\left(F^{\prime}\right)}$. (The reader may well prefer to picture copies of $F^{\prime}$ and $F$ in $G_{n}$ rather than homomorphisms. In fact, it is better to picture embeddings, i.e., labelled copies. There are essentially the same number of these as of homomorphisms.) Since $\operatorname{hom}\left(F, G_{n}\right)$ is the sum over $\phi_{F^{\prime}}$ of the number of extensions, we have

$$
\operatorname{hom}\left(F, G_{n}\right)=\operatorname{hom}\left(F^{\prime}, G_{n}\right) \mathbb{E}\left(Z_{n}\left(F^{\prime}, F\right)\right) n^{k-\ell} p^{e(F)-e\left(F^{\prime}\right)},
$$

and hence

$$
t_{p}\left(F, G_{n}\right)=t_{p}\left(F^{\prime}, G_{n}\right) \mathbb{E}\left(Z_{n}\left(F^{\prime}, F\right)\right) .
$$

For $r \geq 2$, let $r F / F^{\prime}$ denote the graph formed by the union of $r$ copies of $F$ which all meet in the same subgraph $F^{\prime}$, so $r F / F^{\prime}$ has $\left|F^{\prime}\right|+r\left(|F|-\left|F^{\prime}\right|\right)$ vertices and $e\left(F^{\prime}\right)+r\left(e(F)-e\left(F^{\prime}\right)\right)$ edges. A homomorphism from $r F / F^{\prime}$ to $G_{n}$ consists of a homomorphism $\phi$ from $F^{\prime}$ to $G_{n}$ together with $r$ extensions of $\phi$ to homomorphisms from $F$ to $G$, which may or may not be distinct. (They almost always will be.) Since we have normalized by the right powers of $n$ and $p$, it follows that

$$
t_{p}\left(r F / F^{\prime}, G_{n}\right)=t_{p}\left(F^{\prime}, G_{n}\right) \mathbb{E}\left(Z_{n}\left(F^{\prime}, F\right)^{r}\right) .
$$

Let $\mu_{F}=\mu_{F}(n)=n^{|F|} p^{e(F)}$ be the expected number of homomorphisms from $F$ into $G(n, p)$. Then, under the assumptions of any of Conjectures 3.3 3.4 and 3.9, it is easy to see that for $F^{\prime} \subset F$, any $o\left(\mu_{F^{\prime}}\right)$ copies of $F^{\prime}$ meet $o\left(\mu_{F}\right)$ copies of $F$. (Here 'copies' may be subgraphs of $G_{n}$, embeddings, or homomorphisms; it makes no difference.) Otherwise $t_{p}\left(2 F / F^{\prime}, G_{n}\right)$ would not remain bounded. This rules out any construction of a potential counterexample similar to those above; it also shows that if $t_{p}\left(K_{2}, G_{n}\right) \rightarrow 0$ and Assumption 3.1 holds (i.e., $\left(G_{n}\right)$ has bounded subgraph counts), then $t_{p}\left(F, G_{n}\right) \rightarrow 0$ for every $F$.

Conjecture 3.9 states that infinitely many conclusions (one for each $F$ ) hold under the same assumptions. We have already proved some of these conclusions, 
with $F=C_{k}, k \geq 5$. Our next aim is to prove a corresponding result for a much wider class of graphs. In doing so, the following observation will be useful.

Lemma 3.15. Let $X_{n} \geq 0$ be a sequence of random variables with $\sup _{n} \mathbb{E}\left(X_{n}^{k}\right)<$ $\infty$ for every $k \geq 1$. Then $\mathbb{E}\left(X_{n}^{k}\right) \rightarrow 1$ for every $k$ if and only if $X_{n} \stackrel{\mathrm{p}}{\rightarrow} 1$.

Proof. The forward implication is totally trivial: $\mathbb{E}\left(X_{n}\right) \rightarrow 1$ and $\mathbb{E}\left(X_{n}^{2}\right) \rightarrow 1$ already imply $X_{n} \stackrel{\mathrm{p}}{\rightarrow} 1$. The reverse implication is not much harder. Suppose that $X_{n} \stackrel{\mathrm{p}}{\rightarrow} 1$, but that $\mathbb{E}\left(X_{n}^{k}\right) \not \rightarrow 1$ for some $k$. For any $M$, the variables $X_{n}^{k} 1_{X_{n} \leq M}$ are uniformly bounded and converge in probability to 1 , so $\mathbb{E}\left(X_{n}^{k} 1_{X_{n} \leq M}\right) \rightarrow 1$. It follows that there is some $M(n) \rightarrow \infty$ such that $\mathbb{E}\left(X_{n}^{k} 1_{X_{n} \leq M(n)}\right) \rightarrow 1$. But then $\mathbb{E}\left(X_{n}^{k} 1_{X_{n}>M(n)}\right) \not \nrightarrow 0$, so

$$
\mathbb{E}\left(X_{n}^{k+1}\right) \geq \mathbb{E}\left(X_{n}^{k+1} 1_{X_{n}>M(n)}\right) \geq M(n) \mathbb{E}\left(X_{n}^{k} 1_{X_{n}>M(n)}\right)
$$

is unbounded, contradicting our assumptions.

Corollary 3.16. Under the assumptions of Conjecture 3.9, if $F^{\prime} \subset F$ and $t_{p}\left(F^{\prime}, G_{n}\right) \rightarrow 1$, then $Z_{n}\left(F^{\prime}, F\right) \stackrel{\mathrm{p}}{\rightarrow} 1$ if and only if $t_{p}\left(r F / F^{\prime}, G_{n}\right) \rightarrow 1$ for every $r \geq 1$.

Proof. Apply Lemma 3.15 to the random variable $Z_{n}\left(F^{\prime}, F\right)$, using (22) to evaluate its moments.

We shall say that the distribution of $F$ is flat over that of $F^{\prime}$ in $G_{n}$, or simply that $F$ is flat over $F^{\prime}$ if $Z_{n}\left(F^{\prime}, F\right) \stackrel{\mathrm{p}}{\rightarrow} 1$.

Lemma 3.17. Under the assumptions of Conjecture 3.9 we have $s_{p}\left(K_{s, t}, G_{n}\right) \rightarrow$ 1 for all $s, t \geq 1$. Moreover, $K_{1, s}$ is flat over $E_{s}$, where $E_{s}$ is the empty subgraph of $K_{1, s}$ induced by the vertices in the second part.

Proof. Let $d_{1}, \ldots, d_{n}$ denote the degrees of the vertices of $G_{n}$, and $\bar{d}$ the average degree. Fix $s \geq 1$. By convexity, we have

$$
\operatorname{hom}\left(K_{1, s}, G_{n}\right)=\sum_{i=1}^{n} d_{i}^{s} \geq n \bar{d}^{s},
$$

which we can rewrite as $t_{p}\left(K_{1, s}, G_{n}\right) \geq t_{p}\left(K_{2}, G_{n}\right)^{s}$. Since $t_{p}\left(K_{2}, G_{n}\right) \rightarrow 1$ by assumption, this gives

$$
t_{p}\left(K_{1, s}, G_{n}\right) \geq 1+o(1) .
$$

Specializing to $s=2$ for the moment, let $Z_{n}=Z_{n}\left(E_{2}, K_{1,2}\right)$ be the random variable describing the distribution of the number of common neighbours of a random pair of vertices of $G_{n}$. For any empty graph $E_{k}$ we have $t_{p}\left(E_{k}, G_{n}\right)=1$. Hence, from (22) and (23),

$$
\mathbb{E}\left(Z_{n}\right)=t_{p}\left(K_{1,2}, G_{n}\right) \geq 1+o(1)
$$


On the other hand, since $t K_{1,2} / E_{2}=K_{2, t}$,

$$
\mathbb{E}\left(Z_{n}^{2}\right)=t_{p}\left(K_{2,2}, G_{n}\right)=t_{p}\left(C_{4}, G_{n}\right) \rightarrow 1 .
$$

Hence, $Z_{n} \stackrel{\text { p }}{\rightarrow}$ 1, i.e., $K_{1,2}$ is flat over pairs of vertices. By Corollary 3.16 we thus have $t_{p}\left(K_{2, t}, G_{n}\right) \rightarrow 1$ for every $t$.

Returning to general $s$, let $W_{n}=Z_{n}\left(E_{s}, K_{1, s}\right)$. From (23) we have $\mathbb{E}\left(W_{n}\right)=$ $t_{p}\left(K_{1, s}, G_{n}\right) \geq 1+o(1)$. But we have just shown that $\mathbb{E}\left(W_{n}^{2}\right)=t_{p}\left(K_{2, s}, G_{n}\right) \rightarrow$ 1 , so $W_{n} \stackrel{\text { p }}{\rightarrow}$ 1, i.e., $K_{1, s}$ is flat over $E_{s}$. Applying Corollary 3.16 again we thus have $t_{p}\left(K_{s, t}, G_{n}\right) \rightarrow 1$ for every $t$, as required.

Theorem 3.18. Let $F$ be any fixed graph with girth at least 4 , and let $F^{\prime} \neq F$ be any induced subgraph of $F$. Under the assumptions of Conjecture 3.9, $F$ is flat over $F^{\prime}$. Furthermore, $s_{p}\left(F, G_{n}\right), t_{p}\left(F, G_{n}\right) \rightarrow 1$ as $n \rightarrow \infty$.

Proof. Note first that the definition of $Z_{n}\left(F^{\prime}, F\right)$ makes perfect sense when $F^{\prime}$ is the empty 'graph' with no vertices; there is one homomorphism from $F^{\prime}$ to $G_{n}$, and $Z_{n}\left(F^{\prime}, F\right)$ is constant and takes the value $t_{p}\left(F, G_{n}\right)$. Hence, $F$ is flat over the empty subgraph means exactly that $t_{p}\left(F, G_{n}\right) \rightarrow 1$. Since $p=n^{-o(1)}$, we have $s_{p}\left(F, G_{n}\right) \sim t_{p}\left(F, G_{n}\right)$, so it suffice to prove the first statement.

We prove the first statement of the theorem by induction on $|F|$. If $|F|=1$, there is nothing to prove. Suppose then that $F$ and $F^{\prime}$ are given, with $|F| \geq 2$, and that the result holds for all smaller $F$.

Suppose first that $F^{\prime}=F-v$ for some vertex $v$ of $F$. Let $E_{s}$ denote the subgraph of $F^{\prime}$ induced by the neighbours of $v$, noting that $E_{s}$ has no edges, as $F$ is triangle free. Set $X_{n}=Z_{n}\left(E_{s}, F^{\prime}\right)$ and $Y_{n}=Z_{n}\left(E_{s}, K_{1, s}\right)$. Note that these random variables are defined on the same probability space: the elements of this space are simply s-tuples of vertices of $G_{n}$. If $F^{\prime}=E_{s}$, then $F^{\prime}$ is trivially flat over $E_{s}$. If not, then $F^{\prime}$ is flat over $E_{s}$ by the induction hypothesis. Hence, in either case, $\mathbb{E}\left(X_{n}^{k}\right) \rightarrow 1$ for every $k$. By the last part of Lemma 3.17 $K_{1, s}$ is flat over $E_{s}$, so $\mathbb{E}\left(Y_{n}^{k}\right) \rightarrow 1$ for every $k$. Thus, by Cauchy-Schwartz,

$$
\mathbb{E}\left(\left(X_{n}-1\right)^{k}\left(Y_{n}-1\right)^{\ell}\right) \leq \sqrt{\mathbb{E}\left(\left(X_{n}-1\right)^{2 k}\right) \mathbb{E}\left(\left(Y_{n}-1\right)^{2 \ell}\right)} \rightarrow 0
$$

for every $k$ and $\ell$, so $\mathbb{E}\left(X_{n}^{k} Y_{n}^{\ell}\right) \rightarrow 1$.

Any homomorphism $\phi_{F^{\prime}}$ from $F^{\prime}$ into $G_{n}$ is the extension of a unique homomorphism $\phi_{E_{s}}$ from $E_{s}$ into $G_{n}$. Furthermore, to extend $\phi_{F^{\prime}}$ to $F$ we must choose for the image of $v$ a common neighbour of the vertices in the image of $\phi_{E_{s}}$. Hence, the value of $Z_{n}=Z_{n}\left(F^{\prime}, F\right)$ on $\phi_{F^{\prime}}$ is simply the value of $Y_{n}$ on $\phi_{E_{s}}$. Choosing $\phi_{F^{\prime}}$ uniformly at random, to obtain the correct distribution for $Z_{n}$, the probability of obtaining a particular restriction $\phi_{E_{s}}$ is proportional to the number of extensions of $\phi_{E_{s}}$ to $F^{\prime}$, i.e., to $X_{n}$. Thus the distribution of $Z_{n}$ is that of $Y_{n}$ 'size biased' by $X_{n}$. In particular,

$$
\mathbb{E}\left(Z_{n}^{k}\right)=\frac{\mathbb{E}\left(X_{n} Y_{n}^{k}\right)}{\mathbb{E}\left(X_{n}\right)} \sim 1 / 1=1
$$


Taking $k=1,2$, it follows that $Z_{n} \stackrel{\mathrm{p}}{\rightarrow} 1$, i.e., that $F$ is flat on $F^{\prime}$, as required.

It remains to handle the case $|F|-\left|F^{\prime}\right| \geq 2$. In this case, we can find an induced subgraph $F^{\prime \prime}=F-v$ of $F$ with $F^{\prime} \subset F^{\prime \prime} \subset F$. Note that $t_{p}\left(F^{\prime}, G_{n}\right), t_{p}\left(F^{\prime \prime}, G_{n}\right) \sim 1$ by induction, that $F^{\prime \prime}$ is flat over $F^{\prime}$ by induction, and that $F$ is flat over $F^{\prime \prime}$ by the case treated above. In particular, we certainly have

$$
t_{p}\left(F, G_{n}\right)=t_{p}\left(F^{\prime \prime}, G_{n}\right) \mathbb{E}\left(Z_{n}\left(F^{\prime \prime}, F\right)\right) \sim 1 .
$$

Fix $\varepsilon>0$. Let us call a copy of $F^{\prime \prime}$ (more precisely, a homomorphism from $F^{\prime \prime}$ into $\left.G_{n}\right)$ bad if it has fewer than $(1-\varepsilon) \mu_{F} / \mu_{F^{\prime \prime}}$ extensions to copies of $F$. Since $F$ is flat over $F^{\prime \prime}$ and $t_{p}\left(F^{\prime \prime}, G_{n}\right) \sim 1$, there are fewer than $\varepsilon^{2} \mu_{F^{\prime \prime}}$ bad copies of $F^{\prime \prime}$ if $n$ is large enough. Since each copy of $F^{\prime \prime}$ extends a unique copy of $F^{\prime}$, it follows that at most $\varepsilon \mu_{F^{\prime}}$ copies of $F^{\prime}$ have more than $\varepsilon \mu_{F^{\prime \prime}} / \mu_{F^{\prime}}$ extensions to bad copies of $F^{\prime \prime}$.

Let $\mathcal{B}_{1}$ denote the set of copies of $F^{\prime}$ that have more than $\varepsilon \mu_{F^{\prime \prime}} / \mu_{F^{\prime}}$ extensions to bad copies of $F^{\prime \prime}$, so $\left|\mathcal{B}_{1}\right| \leq \varepsilon \mu_{F^{\prime}}$ if $n$ is large. Let $\mathcal{B}_{2}$ denote the set of copies of $F^{\prime}$ that have fewer than $(1-\varepsilon) \mu_{F^{\prime \prime}} / \mu_{F^{\prime}}$ extensions to copies of $F^{\prime \prime}$. Since $F^{\prime \prime}$ is flat over $F^{\prime}$, we have $\left|\mathcal{B}_{2}\right| \leq \varepsilon \mu_{F^{\prime}}$ if $n$ is large enough, which we assume from now on. If $\phi$ is a copy of $F^{\prime}$ not in $\mathcal{B}_{1} \cup \mathcal{B}_{2}$, then $\phi$ has at least $(1-2 \varepsilon) \mu_{F^{\prime \prime}} / \mu_{F^{\prime}}$ extensions to good copies of $F^{\prime \prime}$, which in turn have at least $(1-\varepsilon) \mu_{F} / \mu_{F^{\prime}}$ extensions to copies of $F$, so the value of $Z_{n}\left(F^{\prime}, F\right)$ on $\phi$ is at least $(1-2 \varepsilon)(1-\varepsilon)$. Since there are $(1+o(1)) \mu_{F^{\prime \prime}}$ copies of $F^{\prime \prime}$, and $\varepsilon>0$ was arbitrary, it follows that the negative part of $Z_{n}\left(F^{\prime}, F\right)-1$ tends to zero in probability. Since $\mathbb{E}\left(Z_{n}\left(F^{\prime}, F\right)\right)=t_{p}\left(F, G_{n}\right) / t_{p}\left(F^{\prime}, G_{n}\right) \rightarrow 1$, it follows that $Z_{n}\left(F^{\prime}, F\right) \stackrel{\text { p }}{\rightarrow}$, i.e., that $F$ is flat over $F^{\prime}$.

The reader may find many of the arguments above familiar from the dense case; for example, the proof for $K_{2, t}$ is an absolutely standard convexity argument. The key point is that many arguments for the dense case do not carry over. In particular, we have shown that almost all, i.e., all but $o\left(n^{2}\right)$, pairs of vertices have about the right number of common neighbours. In the dense case, it follows immediately that almost all (all but $o\left(p n^{2}\right)=o\left(n^{2}\right)$ ) edges are in the right number of triangles, and hence that $t_{p}\left(K_{3}, G_{n}\right) \rightarrow 1$. Similarly, the proof above shows that any $F$ is flat over all its subgraphs in the dense case, without restriction to girth at least 4 . In the sparse case, there are only $o\left(n^{2}\right)$ edges, and there seems to be no simple way to rule out the possibility that many, or even all, of these pairs of vertices fall in the $o\left(n^{2}\right)$ set with too few common neighbours. Nevertheless, we conjecture that this cannot happen. The simplest graph for which we cannot prove the conclusion of Conjecture 3.9 is the triangle.

Conjecture 3.19. Under the conditions of Conjecture 3.9 we have $s_{p}\left(K_{3}, G_{n}\right) \rightarrow$ 1.

In fact, we do not even have a proof that $G_{n}$ must contain at least one triangle (for $n$ large enough)! 


\subsection{Extensions to smaller $p$.}

Let us return to the study of general subgraphs $F$, rather than simply triangles. If true, the various conjectures above may extend to smaller values of $p$, but one must be careful. Firstly, $s_{p}$ and $t_{p}$ no longer coincide, as noted above. One should work with $s_{p}$, because these quantities behave in the right way for $G_{p}(n, \kappa)$, while $t_{p}$ does not. A simple modification of the proof of Lemma 3.17 considering the distribution of the number of common neighbours of a set of $s$ distinct vertices, shows that if $n p^{s} \rightarrow \infty$, then $s_{p}\left(K_{2}, G_{n}\right) \rightarrow 1, s_{p}\left(C_{4}, G_{n}\right) \rightarrow 1$ and $s_{p}\left(K_{s, t+1}, G_{n}\right)$ bounded together imply $s_{p}\left(K_{s, t}, G_{n}\right) \rightarrow 1$. Taking $p=n^{-\alpha}$, with $0<\alpha<1 / 2$ constant, there is no corresponding result for $t_{p}$, even with $s=2$. Indeed, if $t_{p}\left(K_{2}, G_{n}\right)=1$, then there are at least $n^{t+1} p^{t}$ homomorphisms from $K_{2, t}$ into $G_{n}$ mapping the two vertices in the smaller class to the same vertex. It follows that $t_{p}\left(K_{2, t}, G_{n}\right)$ will be unbounded for any $t>1 / \alpha$.

Secondly, even working with $s_{p}$ rather than $t_{p}$, it makes sense only to consider certain fixed graphs $F$, so we should modify the conjectures to refer to a certain set of 'admissible' $F$. For example, we cannot take $F=C_{4}$ unless $p / n^{-1 / 2} \rightarrow \infty$. Indeed, if $p / n^{-1 / 2} \nrightarrow \rightarrow \infty$, then there are graphs $G_{n}$ with $p n^{2}$ edges but too few $C_{4} \mathrm{~S}$, giving $s_{p}\left(K_{2}, G_{n}\right)=1$ and $\liminf s_{p}\left(C_{4}, G_{n}\right)<1$. Since $s\left(C_{4}, \kappa\right) \geq$ $s\left(K_{2}, \kappa\right)^{4}$ for any $\kappa$, we cannot have $s_{p}\left(F, G_{n}\right) \rightarrow s(F, \kappa)$ for $F=K_{2}$ and for $F=C_{4}$ in this case.

In fact, we should only consider subgraphs $F$ for which the expected number $\mu_{F}=n^{|F|} p^{e(F)}$ of copies of $F$ in $G(n, p)$ is much larger than the number $n^{2} p$ of edges, at least if $p / n^{-1 / 2} \rightarrow \infty$. To see this, first suppose that $n^{|F|} p^{e(F)} \sim$ $A n^{2} p$, for some constant $0<A<\infty$. Form a graph $G^{\prime}$ from $G=G(n, p)$ by adding $\varepsilon n^{2} p /(2 e(F))$ copies $F_{1}, F_{2}, \ldots$ of $F$, chosen uniformly at random from all subgraphs of $K_{n}$ isomorphic to $F$. After deleting the small number of duplicate edges, we have added around $\varepsilon n^{2} p / 2$ edges, so $s_{p}\left(K_{2}, G^{\prime}\right) \sim 1+\varepsilon$. It is easy to check that the number of $C_{4} \mathrm{~s}$ in $G^{\prime}$ containing two or more edges from one single $F_{i}$ is negligible and thus, considering $C_{4}$ s formed from all combinations of edges from $G(n, p)$ and from different $F_{i}$, that $s_{p}\left(C_{4}, G^{\prime}\right) \sim(1+\varepsilon)^{4}$ whp. Hence, the appropriate limiting kernel is the constant kernel $\kappa=1+\varepsilon$. Copies of $F$ itself containing at most one edge from each $F_{i}$ contribute $(1+\varepsilon)^{e(F)}$ to $s_{p}\left(F, G^{\prime}\right)$, but there are $\Theta\left(n^{|F|} p^{e(F)}\right)$ extra copies of $F$, namely the $F_{i}$ themselves. It follows that $s_{p}\left(F, G_{n}\right) \not \rightarrow 1$. If $n^{|F|} p^{e(F)}=o\left(n^{2} p\right)$, then the argument is much simpler: adding a few copies of $F$ to $G(n, p)$ does not change the number of edges or $C_{4} \mathrm{~s}$ significantly, but does change the number of copies of $F$.

We can go somewhat further: the construction in Example 3.11 shows that for $C_{3}$ to be admissible, the expected number of $C_{3}$ s per edge should be larger than $\log n$. A similar construction can be carried out for any fixed $F$, and shows that, at least for suitable balanced $F$, we should require $n^{|F|} p^{e(F)} /\left(n p^{2} \log n\right) \rightarrow$ $\infty$ for $F$ to be admissible. In general, for $F$ to be admissible, we need all induced subgraphs $F^{\prime}$ of $F$ to be admissible; otherwise, the distribution of copies of $F$ over $F^{\prime}$ cannot be flat as we expect in the uniform case.

In the light of the comments above, perhaps the strongest sensible extension of Conjecture 3.19 to smaller $p$ would be the following. 
Conjecture 3.20. Suppose that $p=p(n)=\omega(\sqrt{\log n} / \sqrt{n})$, and that $G_{n}$ is a sequence of graphs with $\left|G_{n}\right|=n$ such that $s_{p}\left(K_{2}, G_{n}\right) \rightarrow 1, s_{p}\left(C_{4}, G_{n}\right) \rightarrow 1$, and $\sup _{n} s_{p}\left(K_{2, t}, G_{n}\right)<\infty$ for each $t$. Then $s_{p}\left(C_{3}, G_{n}\right) \rightarrow 1$.

There is a potential pitfall in handling subgraph counts when $p$ is smaller than $n^{-o(1)}$ : in proving that $s_{p}\left(F, G_{n}\right) \rightarrow 1$ for various graphs $F$ above, we made use of the assumption that $s_{p}\left(F^{\prime}, G_{n}\right)$ is bounded for other graphs $F^{\prime}$. In particular, with $F=K_{2, t}$, we used this assumption for $F^{\prime}=K_{2, t+1}$. It may be that $F^{\prime}$ is admissible whenever $F$ is (as is likely in this case: $K_{2, t}$ should be admissible as soon as $C_{4}$ is), but perhaps not. In the latter case we may be forced to work with a larger admissible set for which we impose the hypothesis of Conjecture 3.3 (or Conjecture 3.4), and a smaller set for which we obtain the conclusion. In any case, the (smaller) admissible set should have the following property: if $\mathcal{F}_{\alpha}$ denotes the set of admissible graphs when $p=n^{-\alpha}, \alpha>0$, then the sets $\mathcal{F}_{\alpha}$ should increase as $\alpha$ decreases, and their union should contain all finite graphs. We shall return to this question in Section 5, in particular in Subsections 5.3 and 5.4, where we prove results that are steps towards (nonuniform) versions of the various conjectures in this section.

\section{Szemerédi's Lemma and the cut metric}

In the next section we shall discuss the relationship between the cut and count metrics. As in the dense case, a key tool in the study of the cut metric is some variant of Szemerédi's Lemma: this will be discussed in this section. Unlike in the dense case, we need an assumption on the graphs we consider to make this useful; roughly speaking, our assumption is that no subgraph of $G_{n}$ containing a constant fraction of the vertices has density more than a constant factor larger than it should have. Several of the usual proofs of Szemerédi's Lemma extend easily to the sparse case under this assumption; this was noted independently by Kohayakawa and Rödl; see [33]. (The much earlier Theorem 2 of Kohayakawa 32 is slightly different.)

Throughout this section, $p=p(n)$ with $p=o(1)$ and $n p \rightarrow \infty$. (Often, $n^{2} p \rightarrow \infty$ is enough in the proofs, but see Remark 4.4) As before, $\left(G_{n}\right)$ always denotes a sequence of graphs with $\left|G_{n}\right|=n$, which need not be defined for all $n$, but only for some infinite set.

For disjoint sets $A, B$ of vertices of a graph $G=G_{n}$ with $n$ vertices, we write $e_{G}(A, B)$ for the number of edges of $G$ joining $A$ to $B$, and

$$
d_{p}(A, B)=\frac{e_{G}(A, B)}{p|A||B|}
$$

for the normalized density of $G$ between $A$ and $B$. It is convenient to extend this definition to sets $A$ and $B$ that need not be disjoint: in this case, we write $e_{G}(A, B)$ for the number of ordered pairs $(i, j)$ with $i \in A, j \in B$ and $i j \in E(G)$; we then define $d_{p}(A, B)$ as above. Note that $e_{G}(A, A)=2 e(G[A])$. We shall also assume that the sequence $\left(G_{n}\right)$ has bounded density in the following sense. 
Assumption 4.1 (bounded density). There is a constant $C$ and a function $n_{0}(\varepsilon)$ such that, for every $\varepsilon>0$ and $n \geq n_{0}(\varepsilon)$, and any $A, B \subset V\left(G_{n}\right)$ with $|A|,|B| \geq \varepsilon n$, we have $d_{p}(A, B) \leq C+\varepsilon$.

It suffices to impose this assumption only when $A=B$, replacing $C$ by $C / 2$ and $\varepsilon$ by $\varepsilon / 2$. Indeed, if $|A|,|B| \geq \varepsilon n, n \geq n_{0}(\varepsilon)$, and $d_{p}(A, B)>C+\varepsilon$ then, by averaging, we may find $A^{\prime} \subset A$ and $B^{\prime} \subset B$ with $\left|A^{\prime}\right|=\left|B^{\prime}\right|=\lceil\varepsilon n\rceil$ such that $d_{p}\left(A^{\prime}, B^{\prime}\right)>C+\varepsilon$. Then $e_{G}\left(A^{\prime} \cup B^{\prime}, A^{\prime} \cup B^{\prime}\right) \geq 2 e_{G}\left(A^{\prime}, B^{\prime}\right)>2(C+\varepsilon)\left|A^{\prime}\right|^{2} \geq$ $(C / 2+\varepsilon / 2)\left|A^{\prime} \cup B^{\prime}\right|^{2}$.

The condition above may be written more compactly as follows:

$$
\forall \varepsilon>0: \limsup _{n \rightarrow \infty} \max \left\{d_{p}(A, B): A, B \subset V\left(G_{n}\right),|A|,|B| \geq \varepsilon n\right\} \leq C .
$$

Note that we shall often assume that (24) holds for a particular value of $C$ : in this case, we say that $\left(G_{n}\right)$ has density bounded by $C$. This is the reason for including the final $+\varepsilon$ in Assumption 4.1 .

It will be convenient to phrase the proof of Szemerédi's Lemma in terms of kernels. In this sparse setting, the way in which we associate a kernel to a graph is different from in the dense case. Indeed, our aim is that the random graph $G(n, p)$ should approximate the constant kernel taking value 1 . For this reason, to a graph $G$ with $n$ vertices $1,2, \ldots, n$ we associate the kernel $\kappa_{G}$ taking the value $1 / p$ on each square $((i-1) / n, i / n] \times((j-1) / n, j / n]$ whenever $i j \in E(G)$, and zero elsewhere. This association will often be implicit: for example, given a graph $G$ and a kernel $\kappa$, we write $d_{\text {cut }}(G, \kappa)$ for $d_{\text {cut }}\left(\kappa_{G}, \kappa\right)$.

The following observation shows the importance of bounded density. In the proof, and throughout this section, given a subset $A$ of the vertices of a graph $G$, we shall often abuse notation by also writing $A$ for the corresponding subset of $[0,1]$.

Lemma 4.2. Let $p=p(n)$ be any function of $n$, let $\kappa:[0,1]^{2} \rightarrow[0, C]$ be a kernel, and let $\left(G_{n}\right)$ be a sequence of graphs with $\left|G_{n}\right|=n$ and $d_{\text {cut }}\left(G_{n}, \kappa\right) \rightarrow 0$. Then $\left(G_{n}\right)$ has density bounded by $C$.

Proof. Suppose that $\left(G_{n}\right)$ does not have density bounded by $C$. Then there is an $\varepsilon>0$ such that, for infinitely many $n$, there are sets $A_{n}, B_{n} \subset V\left(G_{n}\right)$ with $\left|A_{n}\right|,\left|B_{n}\right| \geq \varepsilon n$ and $d_{p}\left(A_{n}, B_{n}\right) \geq C+\varepsilon$. Identifying $A_{n}$ and $B_{n}$ with subsets of $[0,1]$, and writing $\mu$ for Lebesgue measure, we have

$$
\int_{A_{n} \times B_{n}} \kappa_{G_{n}}=d_{p}\left(A_{n}, B_{n}\right) \mu\left(A_{n}\right) \mu\left(B_{n}\right) \geq(C+\varepsilon) \mu\left(A_{n}\right) \mu\left(B_{n}\right) .
$$

Since $\kappa$ is bounded by $C$, it follows that

$$
\left|\int_{A_{n} \times B_{n}} \kappa_{G_{n}}-\kappa^{(\tau)}\right| \geq \varepsilon \mu\left(A_{n}\right) \mu\left(B_{n}\right) \geq \varepsilon^{3}
$$

for any rearrangement $\kappa^{(\tau)}$ of $\kappa$, which contradicts $d_{\text {cut }}\left(G_{n}, \kappa\right) \rightarrow 0$. 


\subsection{Weakly regular partitions}

If $G$ is a graph with vertex set $\{1,2, \ldots, n\}$, and $\Pi=\left(P_{1}, \ldots, P_{k}\right)$ is a partition of $V(G)$, then we write $G / \Pi$ for the kernel on $[0,1]^{2}$ taking the value $d_{p}\left(P_{a}, P_{b}\right)$ on the union of the squares $((i-1) / n, i / n] \times(j-1) / n, j / n], i \in P_{a}, j \in P_{b}$. We say that a partition $\Pi$ of a graph $G$ is weakly $(\varepsilon, p)$-regular if $\left\|\kappa_{G}-G / \Pi\right\|_{\text {cut }} \leq \varepsilon$. Note that the normalizing function $p$ comes in via the definition of the kernels $\kappa_{G}$ and $G / \Pi$.

For a kernel $\kappa$, the definitions are similar: for $A, B \subset[0,1]$ we write $\kappa(A, B)$ for the integral of $\kappa$ over $A \times B$, and

$$
d(A, B)=d_{\kappa}(A, B)=\frac{\kappa(A, B)}{\mu(A) \mu(B)}
$$

for the average value of $\kappa$ on $A \times B$. Then $d_{p}(A, B)$, defined using $G$, is exactly $d(A, B)$, defined using $\kappa_{G}$, so the kernel $G / \Pi$ is obtained from $\kappa_{G}$ by replacing the value at each point by the average over the relevant rectangle $P_{a} \times P_{b}$. For $\kappa$ a kernel and $\Pi$ a partition of $[0,1]^{2}$, we define $\kappa / \Pi$ similarly. The partition $\Pi$ is weakly $\varepsilon$-regular with respect to $\kappa$ if $\left.\|\kappa-\kappa / \Pi\|\right|_{\text {cut }} \leq \varepsilon$.

The next lemma is a a sparse equivalent of (a version of) the Frieze-Kannan 'weak' form of Szemerédi's Lemma from 27. As with many proofs of the various forms of Szemerédi's Lemma, the proof of the dense result is not hard to adapt to the sparse setting: the only additional complication is that one must make sure that the parts of the partition remain large enough so that we can make use of the bounded density assumption. In the following lemma, $p=p(n)$ is any normalizing function with $p n^{2} \rightarrow \infty$. In principle, the various constants depend on the choice of $p$, but this is not the case if we impose an explicit lower bound on $p(n)$, such as the harmless bound $p n^{3 / 2} \rightarrow \infty$.

Lemma 4.3. Let $p=p(n)$ be any function with $0<p \leq 1$ and $p n^{2} \rightarrow \infty$. Let $\varepsilon>0, C>0$ and $k \geq 1$ be given. There exist constants $n_{0}, K$ and $\eta>0$, all depending on $\varepsilon, C$ and $k$, such that, if $G_{n}$ is any graph with $n \geq n_{0}$ vertices such that

$$
d_{p}(A, B) \leq C \text { whenever }|A|,|B| \geq \eta n
$$

and $\Pi$ is any partition of $V(G)$ into $k$ parts $P_{1}, \ldots, P_{k}$ with sizes as equal as possible, then there is a weakly $(\varepsilon, p)$-regular partition $\Pi^{\prime}$ of $V\left(G_{n}\right)$ into $K$ parts that refines $\Pi$.

Proof. Let $\Pi_{0}=\Pi$. We inductively define a sequence $\Pi_{t}$ of partitions of $V(G)$ into $k_{t}=2^{t} k$ parts, stopping either when we reach some $\Pi_{t}$ that is weakly $(\varepsilon / 2, p)$-regular, or when $t \geq T=\left\lceil 16^{2} C / \varepsilon^{2}\right\rceil+1$. Every part of $\Pi_{t}$ will have size at least $\gamma^{t} n /(2 k)$, where $\gamma=\varepsilon /(100 C)$. Note that $\Pi_{0}$ satisfies this condition.

Set $\eta=\gamma^{T} /(2 k)$, and let $n_{0}$ be a large constant to be chosen later. We shall write $\kappa_{t}$ for the kernel $G / \Pi_{t}$, noting that, since all parts of $\Pi_{t}$ have size at least $\eta n$, the kernel $\kappa_{t}$ is bounded by $C$.

Given $\Pi_{t}$ as above, suppose that $\Pi_{t}$ is not weakly $(\varepsilon / 2, p)$-regular. Then there is a cut $[0,1]=A \cup A^{\mathrm{c}}$ exhibiting this, i.e., a set $A \subset[0,1]$ for which 
$\left|\kappa_{G}\left(A, A^{\mathrm{c}}\right)-\kappa_{t}\left(A, A^{\mathrm{c}}\right)\right| \geq \varepsilon / 2$. Since both $\kappa_{G}$ and $\kappa_{t}$ correspond to weighted graphs on $V(G)=\{1,2, \ldots, n\}$, we may choose the cut $A$ to correspond to a subset of $V(G)$ : among all 'worst' cuts, there is a cut of this form.

Our aim is to modify $A$ slightly to obtain a set $B$ (which we may think of as a subset of $V(G)$ or as a subset of $[0,1])$ and then take two parts $P_{i} \cap B$ and $P_{i} \cap B^{\mathrm{c}}$ of $\Pi_{t+1}$ for each part of $\Pi_{t}$; in doing so, we must ensure that neither of these parts is too small. We modify the set $A$ to obtain $B$ in $k_{t}$ stages, one for each part $P_{i}$. At each stage, we move a set $S$ of at most $\gamma\left|P_{i}\right| \geq \eta n$ vertices from $A$ to $A^{\mathrm{c}}$ or vice versa, to ensure that both $B$ and $B^{\mathrm{c}}$ meet $P_{i}$ in at least $\gamma\left|P_{i}\right|$ vertices. Since $\kappa_{t}$ is bounded by $C$, this changes the value of the cut $\kappa_{t}\left(A, A^{\mathrm{c}}\right)$ by at most $2 C \gamma\left|P_{i}\right| / n$.

From (25), the set $S$ meets at most $C p n \gamma\left|P_{i}\right|$ edges of $G$ : to see this, apply (25) to $S$ and $V(G)$ if $|S| \geq \eta n$, and to $S^{\prime}$ and $V(G)$ otherwise, for any $S^{\prime} \supset S$ with $\lceil\eta n\rceil$ vertices. Hence, the value of the cut $\kappa_{G}\left(A, A^{\mathrm{c}}\right)$ changes by at most $2 C \gamma\left|P_{i}\right| / n$ when we move our set $S$ from one side of the cut to the other. After all these changes, we have

$$
\left|\kappa_{t}\left(A, A^{\mathrm{c}}\right)-\kappa_{t}\left(B, B^{\mathrm{c}}\right)\right|,\left|\kappa_{G}\left(A, A^{\mathrm{c}}\right)-\kappa_{G}\left(B, B^{\mathrm{c}}\right)\right| \leq 2 C \gamma \leq \varepsilon / 8 .
$$

It follows that

$$
\left|\kappa_{G}\left(B, B^{\mathrm{c}}\right)-\kappa_{t}\left(B, B^{\mathrm{c}}\right)\right| \geq \varepsilon / 4 \text {. }
$$

Let $\Pi_{t+1}$ be the partition obtained by intersecting each part of $\Pi_{t}$ with $B$ and $B^{\mathrm{c}}$, noting that $\Pi_{t+1}$ has all the required properties. Set $\kappa_{t+1}=G / \Pi_{t+1}$, noting that $\kappa_{t+1}\left(B, B^{\mathrm{c}}\right)=\kappa_{G}\left(B, B^{\mathrm{c}}\right)$, since $\Pi_{t+1}$ refines the partition $\left(B, B^{\mathrm{c}}\right)$. From (26) it thus follows that

$$
\left\|\kappa_{t+1}-\kappa_{t}\right\|_{1} \geq\left\|\kappa_{t+1}-\kappa_{t}\right\|_{\text {cut }} \geq \varepsilon / 4
$$

with the final inequality witnessed by the cut $\left(B, B^{\mathrm{c}}\right)$. Hence, $\left\|\kappa_{t+1}-\kappa_{t}\right\|_{2}^{2} \geq$ $\left\|\kappa_{t+1}-\kappa_{t}\right\|_{1}^{2} \geq \varepsilon^{2} / 16$. Since $\kappa_{t}$ may be obtained from $\kappa_{t+1}$ by averaging over rectangles, $\kappa_{t}$ and $\kappa_{t+1}-\kappa_{t}$ are orthogonal (in the sense that their product integrates to zero). Thus,

$$
\left\|\kappa_{t+1}\right\|_{2}^{2}=\left\|\kappa_{t}\right\|_{2}^{2}+\left\|\kappa_{t+1}-\kappa_{t}\right\|_{2}^{2} \geq\left\|\kappa_{t}\right\|_{2}^{2}+\varepsilon^{2} / 16
$$

It follows by induction that $\left\|\kappa_{t}\right\|_{2}^{2} \geq t \varepsilon^{2} / 16$ as long as our construction continues. But, as noted above, $\kappa_{t}$ is bounded by $C$, so our construction must stop after at most $16 C^{2} / \varepsilon^{2}$ steps. Since this number is smaller than $T$, we must stop at a weakly $(\varepsilon / 2, p)$-regular partition.

To complete the proof we modify the final partition $\Pi_{t}$ slightly. Set $K=$ $k\left\lceil\gamma^{-T}\right\rceil$, and note that, since $t \leq T-1$, each part of $\Pi_{t}$ has size at least $\gamma^{-1} n / K$. First, adjust the parts slightly so that the size of each is of the form $a\lfloor n / K\rfloor+b\lceil n / K\rceil, a, b \in \mathbb{Z}^{+}$, replacing the kernel $\kappa_{t}$ by a new kernel $\kappa^{\prime}$ corresponding to the altered partition. Arguing as above, $\left\|\kappa_{t}-\kappa^{\prime}\right\|_{\text {cut }} \leq 2 C \gamma \leq$ $\varepsilon / 4$, so, by the triangle inequality and weak $(\varepsilon / 2, p)$-regularity of $\Pi_{t}$, we have

$$
\left\|\kappa^{\prime}-\kappa_{G}\right\|_{\text {cut }} \leq\left\|\kappa_{t}-\kappa_{G}\right\|_{\text {cut }}+\left\|\kappa_{t}-\kappa^{\prime}\right\|_{\text {cut }} \leq \varepsilon / 2+\varepsilon / 4=3 \varepsilon / 4
$$


Finally, we split each part randomly into parts of sizes exactly $\lfloor n / K\rfloor$ and $\lceil n / K\rceil$. Since there are only a constant number of parts, and we have $\Theta\left(p n^{2}\right)$ edges between any two parts with density at least $\varepsilon / 100$, say, it follows from Chernoff's inequality that if $n$ is large enough, which we enforce by choosing $n_{0}$ suitably, then with probability at least 0.99 the density $d_{p}(A, B)$ between every pair $(A, B)$ of new parts $A$ and $B$ coming from parts $P_{i}$ and $P_{j}$ with $d_{p}\left(P_{i}, P_{j}\right) \geq \varepsilon / 100$ is $d_{p}\left(P_{i}, P_{j}\right)(1+o(1))$. Since the densities $d_{p}\left(P_{i}, P_{j}\right)$ are uniformly bounded by $C$, it follows that with probability at least 0.99 we have $\left\|\kappa^{\prime \prime}-\kappa^{\prime}\right\|_{1} \leq \varepsilon / 100$. But then

$\left\|\kappa^{\prime \prime}-\kappa_{G}\right\|_{\text {cut }} \leq\left\|\kappa^{\prime \prime}-\kappa^{\prime}\right\|_{\text {cut }}+\left\|\kappa^{\prime}-\kappa_{G}\right\|_{\text {cut }} \leq\left\|\kappa^{\prime \prime}-\kappa^{\prime}\right\|_{1}+\left\|\kappa^{\prime}-\kappa_{G}\right\|_{\text {cut }} \leq \varepsilon / 100+\varepsilon / 2$.

so our final partition is indeed weakly $(\varepsilon, p)$-regular.

If for any reason we want a weakly $(\varepsilon, p)$-regular partition into a particular number $K$ of parts (which must be a multiple of the number in the original partition if we are refining a given partition), the proof above gives such a partition for any large enough $K$, indeed, for any $K \geq k\left\lceil\gamma^{-T}\right\rceil$. Of course, $n_{0}$ then depends on $K$.

Remark 4.4. The proof of Lemma 4.3 works even if $p$ is very small, say of order $1 / n$. However, this is of no help - it is impossible for Assumption 4.1 (the sequence version of (25)) to be satisfied in this range, except in a trivial way if $e\left(G_{n}\right)=o\left(p n^{2}\right)$. Indeed, picking any $\varepsilon p n^{2}$ edges of $G_{n}$, and putting one endpoint of each edge into $A$ and the other into $B$, we find sets $A, B$ with $|A|,|B| \leq \varepsilon p n^{2}$ but $e(A, B) \geq \varepsilon p n^{2}$, which gives $d_{p}(A, B) \geq 1 /\left(\varepsilon p^{2} n^{2}\right)$, which tends to infinity as $\varepsilon \rightarrow 0$.

\subsection{Strongly regular partitions}

Usually, when working with the cut metric, weak $\varepsilon$-regularity turns out to be just as good as the usual stronger $\varepsilon$-regularity. In the dense case, this is true also when considering subgraph counts. However, for the subgraph counts we consider in the next section, it turns out that we do in fact need the usual form of $\varepsilon$-regularity.

As usual, a pair $(A, B)$ of (not necessarily disjoint) subsets of $V(G)$ is an $(\varepsilon, p)$-regular pair if $\left|d_{p}\left(A^{\prime}, B^{\prime}\right)-d_{p}(A, B)\right| \leq \varepsilon$ whenever $A^{\prime} \subset A$ and $B^{\prime} \subset B$ satisfy $\left|A^{\prime}\right| \geq \varepsilon|A|$ and $\left|B^{\prime}\right| \geq \varepsilon|B|$. A partition $\Pi=\left(P_{1}, \ldots, P_{k}\right)$ of $V(G)$ is $(\varepsilon, p)$-regular if the parts $P_{i}$ each have size $\lceil n / k\rceil$ or $\lfloor n / k\rfloor$, and all but at most $\varepsilon\left(\begin{array}{c}k \\ 2\end{array}\right)$ of the unordered pairs $\left\{P_{i}, P_{j}\right\}, i \neq j$, are $(\varepsilon, p)$-regular. The definition (now simply of $\varepsilon$-regularity) for a kernel is similar, although here one partitions the interval $[0,1]$ into parts with measure exactly $1 / k$.

The following is (essentially) the sparse version of Szemerédi's Lemma observed by Kohayakawa and Rödl; see [33, where a closely related result is proved. For a proof, see also Gerke and Steger [29]. We shall include a proof here as we state the result in a slightly different way (which makes no real difference), and the use of kernels allows one to phrase the proof a little more simply than in [33] or [29]. 
Lemma 4.5. Let $p=p(n)$ be any function with $0<p \leq 1$ and $p n^{2} \rightarrow \infty$. Let $\varepsilon>0, C>0$ and $k \geq 1$ be given. There exist constants $n_{0}, K$ and $\eta>0$, all depending on $\varepsilon, C$ and $k$, such that, if $G_{n}$ is any graph with $n \geq n_{0}$ vertices such that

$$
d_{p}(A, B) \leq C \text { whenever }|A|,|B| \geq \eta n,
$$

and $\Pi$ is any partition of $V(G)$ into $k$ parts $P_{1}, \ldots, P_{k}$ with sizes as equal as possible, then there is an $(\varepsilon, p)$-regular partition $\Pi^{\prime}$ of $V\left(G_{n}\right)$ into at most $K$ parts that refines $\Pi$.

Proof. Set $\gamma=\varepsilon^{3} /(100 C)$. This time we inductively define a sequence $\Pi_{t}$ of partitions of $V(G)$ into $k_{t}$ parts, where $\Pi_{0}=\Pi, k_{0}=k$, and $k_{t+1}=k_{t}\left\lceil k_{t} 2^{k_{t}} / \gamma\right\rceil$, stopping either when we reach some $\Pi_{t}$ that is $(\varepsilon, p)$-regular, or when $t \geq T=$ $\left\lceil 20 C^{2} / \varepsilon^{5}\right\rceil+1$. The parts of each $\Pi_{t}$ will have sizes as equal as possible. Note that $\Pi_{0}$ satisfies this condition.

Set $\eta=1 /\left(2 k_{T}\right)$, and let $n_{0}$ be a large constant to be chosen later. As before, we write $\kappa_{t}$ for the kernel $G / \Pi_{t}$, noting that, since all parts of $\Pi_{t}$ have size at least $\eta n$, the kernel $\kappa_{t}$ is bounded by $C$.

The key (standard) observation is the following. Let $A$ and $B$ be parts of $\Pi_{t}$, so $\kappa_{t}$ is by definition constant on $A \times B$, and let $A^{\prime} \subset A$ and $B^{\prime} \subset B$. Let $\Pi^{\prime}$ be any partition refining $\Pi$ such that each of $A^{\prime}$ and $B^{\prime}$ is a union of parts of $\Pi^{\prime}$, and let $\kappa^{\prime}=G / \Pi^{\prime}$ be the corresponding kernel. Restricted to $A \times B$, the function $\kappa^{\prime}$ integrates to $d_{p}(A, B) \mu(A) \mu(B)=\int_{A \times B} \kappa_{t}$, since $A$ and $B$ are unions of parts of $\kappa^{\prime}$. Hence, $\kappa_{t}$ and $\kappa^{\prime}-\kappa_{t}$ are orthogonal on this set. Using the fact that $A^{\prime}$ and $B^{\prime}$ are unions of parts of $\kappa^{\prime}$, we see that $\int_{A^{\prime} \times B^{\prime}} \kappa^{\prime}=d_{p}\left(A^{\prime}, B^{\prime}\right) \mu\left(A^{\prime}\right) \mu\left(B^{\prime}\right)$, which differs from the integral of $\kappa_{t}$ over the same set by $\left|d_{p}\left(A^{\prime}, B^{\prime}\right)-d_{p}(A, B)\right| \mu\left(A^{\prime}\right) \mu\left(B^{\prime}\right)$. It follows that $\left\|\kappa^{\prime}-\kappa_{t}\right\|_{2}^{2}$ is at least $\left(d_{p}\left(A^{\prime}, B^{\prime}\right)-d_{p}(A, B)\right)^{2} \mu\left(A^{\prime}\right) \mu\left(B^{\prime}\right)$, and hence, using orthogonality, that

$$
\int_{A \times B}\left(\kappa^{\prime}\right)^{2} \geq \int_{A \times B} \kappa^{2}+\left(d_{p}\left(A^{\prime}, B^{\prime}\right)-d_{p}(A, B)\right)^{2} \mu\left(A^{\prime}\right) \mu\left(B^{\prime}\right) .
$$

Suppose then that $\Pi_{t}$ is not $(\varepsilon, p)$-regular, and let $A_{1}, \ldots, A_{k_{t}}$ denote the parts of $\Pi_{t}$. Then there are at least $\varepsilon\left(\begin{array}{c}k_{t} \\ 2\end{array}\right)$ pairs $\left\{A_{i}, A_{j}\right\}$ of parts of $\Pi_{t}$ that are not $(\varepsilon, p)$-regular. For each, pick sets $A_{i j} \subset A_{i}$ and $A_{j i} \subset A_{j}$ witnessing this, i.e., with $\left|d_{p}\left(A_{i j}, A_{j i}\right)-d_{p}\left(A_{i}, A_{j}\right)\right| \geq \varepsilon$ and $\left|A_{i j}\right| \geq \varepsilon\left|A_{i}\right|,\left|A_{j i}\right| \geq \varepsilon\left|A_{j}\right|$. Let $\Pi^{\prime}$ be the partition whose parts are all atoms formed by the sets $A_{i}$ and the sets $A_{i j}$ taken together, so $\Pi^{\prime}$ refines $\Pi_{t}$, and each $A_{i j}$ is a union of parts of $\Pi^{\prime}$. We could estimate the $L_{2}$-norm of $G / \Pi^{\prime}$ using (28), but this will not be useful if some parts of $\Pi^{\prime}$ are too small, so we first adjust the part sizes.

Define $\Pi_{t+1}$ by dividing each $A_{i}$ into $k_{t+1} / k_{t}$ parts whose sizes are as equal as possible, so that each part of $\Pi^{\prime}$ differs from a union of parts of $\Pi_{t+1}$ in at most $n / k_{t+1}$ vertices: to do this, keep taking for a part of $\Pi_{t+1}$ a subset of some part of $\Pi^{\prime}$, until what is left of every part of $\Pi^{\prime}$ is too small. For each $i$, there are at most $k_{t}$ sets $A_{i j}$ inside $A_{i}$, so $A_{i}$ is a union of at most $2^{k_{t}}$ parts of $\Pi^{\prime}$. It follows that there is some union $A_{i j}^{\prime}$ of parts of $\Pi_{t+1}$ with

$$
\left|A_{i j}-A_{i j}^{\prime}\right| \leq 2^{k_{t}} n / k_{t+1} \leq \gamma n / k_{t}^{2} .
$$


Arguing as in the proof of Lemma 4.3, it follows from (27) that the symmetric difference $S_{i j}$ of $A_{i j}$ and $A_{i j}^{\prime}$ meets at most

$$
C p n\left|S_{i j}\right| \leq C p \gamma n^{2} / k_{t}^{2} \leq \varepsilon^{3} p\left|A_{i}\right|\left|A_{j}\right| / 99
$$

edges of $G$, if $n$ is sufficiently large. Since $\left|S_{i j}\right| \leq \varepsilon^{3}\left|A_{i}\right| / 100$, say, while $\left|A_{i j}\right| \geq$ $\varepsilon\left|A_{i}\right|$ and $\left|A_{j i}\right| \geq \varepsilon\left|A_{j}\right|$, it follows crudely that

$$
\left|d_{p}\left(A_{i j}^{\prime}, A_{j i}^{\prime}\right)-d_{p}\left(A_{i j}, A_{j i}\right)\right| \leq \varepsilon / 2,
$$

which implies that

$$
\left|d_{p}\left(A_{i j}^{\prime}, A_{j i}^{\prime}\right)-d_{p}\left(A_{i}, A_{j}\right)\right| \geq \varepsilon / 2
$$

Now $A_{i j}^{\prime}$ and $A_{j i}^{\prime}$ are unions of parts of $\Pi_{t+1}$, and these sets have size at least $\varepsilon n /\left(2 k_{t}\right)$. Hence, from (28),

$$
\int_{A_{i} \times A_{j}} \kappa_{t+1}^{2} \geq \int_{A_{i} \times A_{j}} \kappa_{t}^{2}+\varepsilon^{4} /\left(16 k_{t}^{2}\right)
$$

for each of the at least $\varepsilon\left(\begin{array}{c}k_{t} \\ 2\end{array}\right)$ irregular pairs $\left\{A_{i}, A_{j}\right\}$. Since $\int_{A_{i} \times A_{j}} \kappa_{t+1}^{2} \geq$ $\int_{A_{i} \times A_{j}} \kappa_{t}^{2}$ always holds, it follows that $\left\|\kappa_{t+1}\right\|_{2}^{2} \geq\left\|\kappa_{t}\right\|_{2}^{2}+\varepsilon^{5} / 20$.

If the construction above does not stop before step $T$, then by induction we have $\left\|\kappa_{t}\right\|_{2} \geq t \varepsilon^{5} / 20$ for $0 \leq t \leq T$. But each $\kappa_{t}$ is bounded by $C$, so $\left\|\kappa_{T}\right\|_{2}^{2} \leq C^{2}$, giving a contradiction. Hence the construction does stop before step $T$, giving an $(\varepsilon, p)$-regular partition with $k_{t} \leq k_{T}$ parts.

Note that Lemma 4.5 implies (essentially) Lemma 4.3 it is easy to check that an $(\varepsilon, p)$-regular partition is, say, weakly $(10(C+1) \varepsilon, p)$-regular, provided the parts are large enough for (27) to hold. However, one of course obtains much worse bounds on the number of parts using the stronger notion of regularity.

Remark 4.6. Let us illustrate once again the difference between the dense and sparse cases with a simple observation. Given a pair $(A, B)$ of sets of vertices of a graph $G$, let $C_{4}(A, B)$ denote the number of homomorphisms from $C_{4}$ into the subgraph spanned by $A \cup B$ mapping a given pair of opposite vertices into $A$ and the other pair into $B$. Standard convexity arguments show that $C_{4}(A, B) \geq d(A, B)^{4}|A|^{2}|B|^{2}$. The pair $(A, B)$ is $(\varepsilon, p)-C_{4}$-minimal if $C_{4}(A, B) \leq\left(d(A, B)^{4}+\varepsilon p^{4}\right)|A|^{2}|B|^{2}$. In the dense case (with $p=1$ ) it is well known and very easy to check that $\varepsilon$-regularity and $\varepsilon-C_{4}$-minimality are essentially equivalent: $\varepsilon$-regularity implies $f(\varepsilon)-C_{4}$-minimality, and $\varepsilon$ - $C_{4}$-minimality implies $g(\varepsilon)$-regularity, for some $f(\varepsilon), g(\varepsilon)$ with $f(\varepsilon), g(\varepsilon) \rightarrow 0$ as $\varepsilon \rightarrow 0$.

Let $\varepsilon>0$ and $M$ be given. By counting $C_{4}$ s it is easy to see that there is a function $f(\varepsilon)$ with $f(\varepsilon) \rightarrow 0$ as $\varepsilon \rightarrow 0$ such that, if $n$ is large enough and $(A, B)$ is $\varepsilon$-regular with $|A|=|B|=n$, then we may partition $A$ and $B$ into sets $A_{1}, \ldots, A_{M}$ and $B_{1}, \ldots, B_{M}$ of almost equal sizes so that every pair $\left(A_{i}, B_{j}\right)$ is $f(\varepsilon)$-regular. Indeed, a random partition has this property with 
probability tending to 1 , since by standard concentration results (for example, the Hoeffding-Azuma inequality), the edge densities and ' $C_{4}$-densities' of the pairs $\left(A_{i}, B_{j}\right)$ are highly concentrated about the corresponding densities for $(A, B)$. It follows immediately that in the usual dense Szemerédi's Lemma, we may specify in advance the number of parts $K$ we would like our partition to have, provided (as in the weak case) that $K$ is large enough given $\varepsilon$, and $n$ large enough given $\varepsilon$ and $K$.

In the sparse case, the fact about random partitioning above is presumably true, but the simple proof using $C_{4}$-counts fails totally. It is still true that $(\varepsilon, p)-C_{4}$-minimality implies $(f(\varepsilon), p)$-regularity, but the reverse implication fails. Indeed, whenever $p=p(n) \rightarrow 0$, given any pair $(A, B)$, we may add a small dense (say complete bipartite) subgraph with two few edges to disturb regularity, but containing many more than $p^{4}|A||B| C_{4}$ s.

\subsection{Szemerédi's lemma and convergence in the cut norm}

We start with a consequence of Lemma 4.3 concerning the cut norm.

Corollary 4.7. Let $\left(G_{n}\right)$ be a sequence of graphs satisfying assumption $C$. Then there is a kernel $\kappa:[0,1]^{2} \rightarrow[0, C]$ and a subsequence $\left(G_{n_{i}}\right)$ of $\left(G_{n}\right)$ with $d_{\text {cut }}\left(G_{n_{i}}, \kappa\right) \rightarrow 0$. Moreover, we may label the vertices of $G_{n_{i}}$ with $1,2, \ldots, n_{i}$ so that $\left\|\kappa_{G_{n_{i}}}-\kappa\right\|_{\text {cut }} \rightarrow 0$.

Proof. We shall only sketch the proof as the argument is exactly the same as that of Lovász and Szegedy for the dense case. Note that given any $\eta>0$ and $\varepsilon>0$, our graphs $G_{n}$ satisfy the assumption (25) of Lemma 4.3 with $C+\varepsilon$ in place of $C$ whenever $n$ is large enough.

First, let us apply Lemma 4.3 with $k=1$ and $\varepsilon=\varepsilon_{1}=1 / 2$, say, to obtain a weakly $\left(\varepsilon_{1}, p\right)$-regular partition $\Pi_{n, 1}$ of $G_{n}$ into $k_{1}=K$ parts, for all large enough $n$. We may relabel the vertices of each $G_{n}$ so that the parts of $\Pi_{n, 1}$ are all intervals. Each kernel $G_{n} / \Pi_{n, 1}$ is characterized by a $k_{1}$ by $k_{1}$ density matrix, whose entries all lie in $\left[0, C+\varepsilon_{1}\right]$. (Indeed, if $k_{1}$ happens to divide $n$, then the kernel is exactly the kernel obtained from the matrix in the obvious way.) Since these matrices live in a compact set, $\left[0, C+\varepsilon_{1}\right]^{k_{1}^{2}}$, they have a convergent subsequence. Passing to the corresponding subsequence of $G_{n}$, we then have $G_{n} / \Pi_{n, 1} \rightarrow \kappa_{1}$ pointwise almost everywhere, and hence in $L_{1}$ and in the cut norm. Since the partitions $\Pi_{1}$ are weakly $\left(\varepsilon_{1}, p\right)$-regular, we have $\left\|\kappa_{G_{n}}-G_{n} / \Pi_{n, 1}\right\|_{\text {cut }} \leq \varepsilon_{1}$. Passing far enough along our subsequence, it follows that $\left\|\kappa_{G_{n}}-\kappa_{1}\right\|_{\text {cut }} \leq 2 \varepsilon_{1}$.

Working within the subsequence defined above, apply Lemma 4.3 again with $\varepsilon=\varepsilon_{2}=1 / 4$, say, and $k=k_{1}$. We find partitions $\Pi_{n, 2}$ refining $\Pi_{n, 1}$, each with $k_{2}$ parts. Relabelling vertices, we may assume that each part of each $\Pi_{n, 2}$ is an interval. (Note that we only reorder the vertices within parts of $\Pi_{n, 1}$.) As before, on a subsequence we have have $G_{n} / \Pi_{n, 2} \rightarrow \kappa_{2}$, for some kernel $\kappa_{2}$ constant on squares of side-length $1 / k_{2}$. Since $\Pi_{n, 2}$ refines $\Pi_{n, 1}$ for each $n$, it follows that the value of $\kappa_{1}$ on each $1 / k_{1}$ by $1 / k_{1}$ square is exactly the average of $\kappa_{2}$ over this set; to see this, let $n \rightarrow \infty$. 
Iterating, we find kernels $\kappa_{1}, \kappa_{2}, \ldots$ each of which can be obtained by averaging the next one, and graphs $G_{n_{i}}$ with $\left\|\kappa_{G_{n_{i}}}-\kappa_{i}\right\|_{\text {cut }} \leq 2 \varepsilon_{i}=2^{1-i}$, say. To complete the proof we simply observe that the sequence $\left(\kappa_{t}\right)$ is a martingale on the state space $[0,1]^{2}$. Since each $\kappa_{t}$ is bounded by $C+\varepsilon_{t}$, by the Martingale Convergence Theorem there is a kernel $\kappa:[0,1]^{2} \rightarrow[0, C]$ with $\kappa_{t} \rightarrow \kappa$ pointwise almost everywhere, and hence in $L_{1}$ and in the cut-norm. Then $\left\|\kappa_{G_{n_{i}}}-\kappa\right\|_{\text {cut }} \rightarrow 0$ as required.

The corollary above says that any (suitable) sequence of graphs has a subsequence converging to a kernel, and is a simple consequence of Szemerédi's Lemma and the Martingale Convergence Theorem. Together with Lemma 4.2 it shows that Assumption 4.1 is the correct assumption to impose on sequences of graphs when we seek limits that are bounded kernels $\kappa:[0,1]^{2} \rightarrow[0, C]$. Before turning to an application of Corollary 4.7 let us note an even simpler consequence of the Martingale Convergence Theorem.

Lemma 4.8. Let $\kappa$ be a bounded kernel, and for $k \geq 1$, let $\kappa_{k}$ be the piecewise constant kernel obtained by dividing $[0,1]^{2}$ into $2^{2 k}$ squares of side $2^{-k}$, and replacing $\kappa$ by its average over each square. Then $\kappa_{k} \rightarrow \kappa$ pointwise almost everywhere and also in $L^{p}$ for any $p$.

Proof. The sequence $\kappa_{k}$ is a bounded martingale on $[0,1]^{2}$, so pointwise convergence is given by the Martingale Convergence Theorem. Since the sequence $\kappa_{k}$ is bounded by $\sup \kappa$, convergence in $L^{p}$ follows by dominated convergence.

A consequence of Corollary 4.7 is that it allows us to compare the two different versions of the cut metric. Recall that for graphs $G_{1}, G_{2}$, we defined $d_{\text {cut }}\left(G_{1}, G_{2}\right)$ by first passing to kernels taking the values 0 and $1 / p$. If $G_{1}$ and $G_{2}$ have the same number of vertices, then there is a more natural definition of their cut-distance, $\widehat{d}_{\text {cut }}\left(G_{1}, G_{2}\right)$, defined in the same way but only allowing rearrangements that 'map whole vertices to whole vertices'. As in the dense case, $d_{\text {cut }}\left(G_{1}, G_{2}\right)$ and $\widehat{d}_{\text {cut }}\left(G_{1}, G_{2}\right)$ are defined by (17) and (8), respectively; the difference between the sparse and dense cases is in the normalization of $\kappa_{G_{i}}$. Writing $d_{\text {cut }}^{1}$ and $\widehat{d}_{\text {cut }}^{1}$ for the metrics defined using $p=1$, Borgs, Chayes, Lovász, Sós and Vesztergombi [17, Theorem 2.3] showed that these metrics are equivalent, proving that

$$
d_{\text {cut }}^{1}\left(G_{1}, G_{2}\right) \leq \widehat{d}_{\text {cut }}^{1}\left(G_{1}, G_{2}\right) \leq 32 d_{\text {cut }}^{1}\left(G_{1}, G_{2}\right)^{1 / 67} .
$$

In fact, they proved (29) for edge-weighted graphs, as long as all edge weights line in $[-1,1]$. Unlike simple Lipschitz equivalence, which may also hold, this does not directly carry over to the sparse setting: we have $d_{\text {cut }}=p^{-1} d_{\text {cut }}^{1}$ and $\widehat{d}_{\text {cut }}=p^{-1} \widehat{d}_{\text {cut }}^{1}$, so (29) can be written as

$$
d_{\text {cut }}\left(G_{1}, G_{2}\right) \leq \widehat{d}_{\text {cut }}\left(G_{1}, G_{2}\right) \leq 32 d_{\text {cut }}\left(G_{1}, G_{2}\right)^{1 / 67} p^{-66 / 67},
$$

which is of little if any use here. However, the equivalence of the two metrics in the sparse case is not too hard to deduce from (29), using Corollary 4.7 
Lemma 4.9. For $i=1,2$, let $\left(G_{n}^{(i)}\right)$ be a sequence of graphs satisfying the bounded density assumption, Assumption 4.1. Then $d_{\mathrm{cut}}\left(G_{n}^{(1)}, G_{n}^{(2)}\right) \rightarrow 0$ if and only if $\widehat{d}_{\text {cut }}\left(G_{n}^{(1)}, G_{n}^{(2)}\right) \rightarrow 0$.

Proof. If $\widehat{d}_{\text {cut }}\left(G_{n}^{(1)}, G_{n}^{(2)}\right) \rightarrow 0$ then, since $d_{\text {cut }} \leq \widehat{d}_{\text {cut }}$, it follows trivially that $d_{\text {cut }}\left(G_{n}^{(1)}, G_{n}^{(2)}\right) \rightarrow 0$.

Suppose now that $d_{\text {cut }}\left(G_{n}^{(1)}, G_{n}^{(2)}\right) \rightarrow 0$; our aim is to show that we have $\widehat{d}_{\text {cut }}\left(G_{n}^{(1)}, G_{n}^{(2)}\right) \rightarrow 0$, so we may suppose that this is not the case. Hence, passing to a subsequence, we may assume that $\widehat{d}_{\text {cut }}\left(G_{n}^{(1)}, G_{n}^{(2)}\right) \geq \delta$ for some positive $\delta$ and all $n$ in our subsequence.

Applying Corollary 4.7 twice, the second time to a suitable subsequence, we find kernels $\kappa_{1}, \kappa_{2}:[0,1]^{2} \rightarrow[0, C]$, and subsequences of the sequences $\left(G_{n}^{(i)}\right)$ defined for the same values of $n$ on which $\left\|\kappa_{G_{n}^{(i)}}-\kappa_{i}\right\|_{\text {cut }} \rightarrow 0$. Since $d_{\text {cut }}\left(G_{n}^{(1)}, G_{n}^{(2)}\right) \rightarrow 0$, it follows that $d_{\text {cut }}\left(\kappa_{1}, \kappa_{2}\right)=0$.

For any $\varepsilon>0$, by Lemma 4.8 we may find a $K$ and kernels $\kappa_{1}^{\prime}, \kappa_{2}^{\prime}:[0,1]^{2} \rightarrow$ $[0, C]$ that are constant on squares of side $1 / K$, with $\left\|\kappa_{i}^{\prime}-\kappa_{i}\right\|_{\text {cut }} \leq \varepsilon$. Since the kernels $\kappa_{i}^{\prime}$ may be thought of as weighted graphs, it would appear that we have gone round in circles, but the point is that they are dense weighted graphs. Regarding the kernels $\kappa_{1}^{\prime} / C$ and $\kappa_{2}^{\prime} / C$ as weighted graphs with edge weights in $[0,1]$, we have

$$
d_{\text {cut }}\left(\kappa_{1}^{\prime} / C, \kappa_{2}^{\prime} / C\right)=d_{\text {cut }}\left(\kappa_{1}^{\prime}, \kappa_{2}^{\prime}\right) / C \leq 2 \varepsilon / C,
$$

so (29) gives

$$
\widehat{d}_{\text {cut }}\left(\kappa_{1}^{\prime}, \kappa_{2}^{\prime}\right)=C \widehat{d}_{\text {cut }}\left(\kappa_{1}^{\prime} / C, \kappa_{2}^{\prime} / C\right) \leq 32 C(2 \varepsilon / C)^{1 / 67} .
$$

Hence, there is a rearrangement of $\kappa_{1}^{\prime}$ preserving intervals that is close to $\kappa_{2}^{\prime}$ in the cut norm. Ignoring divisibility, adapting this rearrangement to the graph $G_{n}^{(1)}, n$ much larger than $K$, and using $\left\|\kappa_{G_{n}^{(i)}}-\kappa_{i}^{\prime}\right\|_{\text {cut }} \leq \varepsilon+o(1)$, it follows that that $\widehat{d}_{\text {cut }}\left(G_{n}^{(1)}, G_{n}^{(2)}\right) \leq O(\varepsilon)+O\left(\varepsilon^{1 / 67}\right)$. Choosing $\varepsilon$ small enough, the final bound is less than $\delta$, contradicting our assumptions.

Corollary 4.7 shows that one property of the cut metric carries over to the sparse setting: for every suitable sequence $\left(G_{n}\right)$, i.e., any sequence satisfying the bounded density assumption, Assumption 4.1 there is a kernel $\kappa$ and a subsequence converging to $\kappa$ in $d_{\text {cut }}$. In the other direction, as in the dense case, such a sequence is given by the natural random construction.

Lemma 4.10. Let $p=p(n)$ satisfy $n p \rightarrow \infty$, let $C>0$ be constant, let $\kappa$ : $[0,1]^{2} \rightarrow[0, C]$ be a bounded kernel, and let $G_{n}=G_{p}(n, \kappa)$. Then $d_{\text {cut }}\left(G_{n}, \kappa\right) \rightarrow$ 0 almost surely. Also, the sequence $\left(G_{n}\right)$ satisfies the bounded density assumption, Assumption [4.1, with probability 1.

Proof. The second statement is essentially immediate from Chernoff's inequality, constructing $G_{n}$ as a subgraph of the Erdös-Rényi random graph $G(n, C p)$; it also follows from the first statement and Lemma 4.2 
We now turn to the proof that $d_{\text {cut }}\left(G_{n}, \kappa\right) \rightarrow 0$. Recall that $\kappa$ is of finite type if $[0,1]$ may be partitioned into sets $A_{1}, \ldots, A_{k}$ so that $\kappa$ is constant on each rectangle $A_{i} \times A_{j}$. We first suppose that $\kappa$ is of finite type. Rearranging $\kappa$, and ignoring parts with measure zero, we may assume that each $A_{i}$ is an interval with positive measure. Recall that $G_{n}=G_{p}(n, \kappa)$ is constructed by first choosing the 'types' $x_{1}, \ldots, x_{n}$ of the vertices independently and uniformly at random from $[0,1]$. Let $n_{i}$ denote the number of vertices of type $i$, noting that we have $n_{i} \sim \mu\left(A_{i}\right) n$ a.s. Let us adjust the intervals $A_{i}$ slightly, replacing $A_{i}$ by a set $A_{i}^{\prime}\left(=A_{i}^{\prime}(n)\right)$ with measure $n_{i} / n$. Let $\kappa^{\prime}=\kappa^{\prime}(n)$ be the adjusted kernel, taking on $A_{i}^{\prime} \times A_{j}^{\prime}$ the value that $\kappa$ takes on $A_{i} \times A_{j}$. Since, a.s., we adjust the length of each $A_{i}$ by $o(1)$, the kernels $\kappa^{\prime}$ and $\kappa$ differ on a set of measure $o(1)$. Since each is bounded, it follows that

$$
\left\|\kappa-\kappa^{\prime}\right\|_{\text {cut }} \leq\left\|\kappa-\kappa^{\prime}\right\|_{1} \rightarrow 0
$$

a.s., as $n \rightarrow \infty$.

Given $x_{1}, \ldots, x_{n}$, let $G^{\prime}$ be the weighted graph in which each edge is present and has weight $w_{i j}=p \kappa\left(x_{i}, x_{j}\right)$. Then, relabelling the vertices so that those with $x_{i}=j$ correspond to the set $A_{j}^{\prime}$, we see that $\kappa_{G^{\prime}}=\kappa^{\prime}$. The graph $G_{n}$ may be constructed from $G^{\prime}$ by simply selecting each edge $i j$ independently, with probability equal to its weight in $G^{\prime}$. As noted earlier, for a kernel corresponding to a (weighted) graph, the cut norm (defined by (2)) is realized by a cut corresponding to a partition of the vertex set, so

$$
\left\|\kappa_{G_{n}}-\kappa^{\prime}\right\|_{\text {cut }}=\left\|\kappa_{G_{n}}-\kappa_{G^{\prime}}\right\|_{\text {cut }}=\max _{S \subset V\left(G_{n}\right)}\left|\frac{e_{G_{n}}\left(S, S^{\mathrm{c}}\right)-\sum_{i \in S, j \in S^{\mathrm{c}}} w_{i j}}{n^{2} p}\right| .
$$

Having conditioned on $x_{1}, \ldots, x_{n}$, for each $S$ the random variable $X=e_{G_{n}}\left(S, S^{\mathrm{c}}\right)$ has mean exactly $\sum_{i \in S, j \in S^{c}} w_{i j}$. Furthermore, $\mathbb{E}(X)=O\left(n^{2} p\right)$. Since $X$ is a sum of independent indicator variables, it follows from the Chernoff bounds (say), that for any $\varepsilon>0$ we have $\mathbb{P}\left(|X-\mathbb{E}(X)| \geq \varepsilon n^{2} p\right) \leq \exp \left(-c_{\varepsilon} n^{2} p\right)$ for some $c_{\varepsilon}>0$. Since $n^{2} p=\omega(n)$, this probability decays superexponentially. Since there are only $2^{n}$ sets $S$ to consider, we see that $\mathbb{P}\left(\left\|\kappa_{G_{n}}-\kappa^{\prime}\right\|_{\text {cut }} \geq \varepsilon\right)$ decays superexponentially as $n \rightarrow \infty$. Since $\varepsilon>0$ was arbitrary, using (30) it follows that $d_{\text {cut }}\left(G_{n}, \kappa\right) \rightarrow 0$ a.s.

So far we assumed that $\kappa$ was of finite type. Given an arbitrary $\kappa$, for each $\varepsilon>0$ we can find a finite type approximation $\kappa_{\varepsilon}$ to $\kappa$ with

$$
\left\|\kappa_{\varepsilon}-\kappa\right\|_{\text {cut }} \leq\left\|\kappa_{\varepsilon}-\kappa\right\|_{1} \leq \varepsilon
$$

see, for example, Lemma 4.8. One can couple the random graphs $G_{n}=G_{p}(n, \kappa)$ and $G_{n}^{\prime}=G_{p}\left(n, \kappa_{\varepsilon}\right)$ using the same vertex types $x_{1}, \ldots, x_{n}$ for each, in such a way that the symmetric difference $G_{n} \Delta G_{n}^{\prime}$ has the distribution of $G_{p}(n, \Delta \kappa)$, where $\Delta \kappa(x, y)=\left|\kappa(x, y)-\kappa_{\varepsilon}(x, y)\right|$. The expected number of edges of $G_{p}(n, \Delta \kappa)$ is simply $n^{2} p\|\Delta \kappa\|_{1} / 2$, which is at most $n^{2} p \varepsilon / 2$. It is easy to check that the actual number is tightly concentrated about the mean, so

$$
d_{\text {cut }}\left(G_{n}, G_{n}^{\prime}\right) \leq\left\|\kappa_{G_{n}}-\kappa_{G_{n}^{\prime}}\right\|_{1}=\frac{2 e\left(G_{n} \Delta G_{n}^{\prime}\right)}{n^{2} p} \leq 2 \varepsilon
$$


holds with probability tending (rapidly) to 1 as $n \rightarrow \infty$. Using the finite-type case to show that $d_{\text {cut }}\left(G_{n}^{\prime}, \kappa_{\varepsilon}\right) \rightarrow 0$ and the bound $d_{\text {cut }}\left(\kappa, \kappa_{\varepsilon}\right) \leq \varepsilon$, and recalling that $\varepsilon>0$ was arbitrary, the result follows.

\section{Comparison between cut and count conver- gence}

Throughout this section, we fix a function $p=p(n)$, and consider sequences $\left(G_{n}\right)$ of graphs with $\left|G_{n}\right|=n$. In the dense case, with $p(n)=1$ for all $n$, Borgs, Chayes, Lovász, Sós and Vesztergombi [17] showed that such a sequence converges to a kernel $\kappa$ in $d_{\text {cut }}$ if and only if it converges to $\kappa$ in $d_{\text {sub }}$; here we wish to investigate whether this result can be extended to the sparse case. To do this, we first have to make sense of the definitions. For $d_{\text {cut }}$, as in the previous section, we simply associate a kernel $\kappa_{n}$ to $G_{n}$ as before, with $\kappa_{n}$ taking the values 0 and $1 / p$. Then we use the usual definition of $d_{\text {cut }}$ for (dense) kernels to define $d_{\text {cut }}\left(G_{n}, G_{m}\right)$ and $d_{\text {cut }}\left(G_{n}, \kappa\right)$. In the light of Lemma 4.9, for questions of convergence the metrics $d_{\text {cut }}$ and $\widehat{d}_{\text {cut }}$ are equivalent; we shall use $d_{\text {cut }}$ rather than $\widehat{d}_{\text {cut }}$ in this section.

\subsection{Admissible subgraphs and their counts}

If $p=n^{-o(1)}$, then we use (15) and (16) to define $d_{\text {sub }}$, so convergence in $d_{\text {sub }}$ is equivalent to convergence of the sequence $s_{p}\left(F, G_{n}\right)$ for each fixed graph $F$. For smaller $p$, as noted in Subsection 3.5 it makes sense only to consider graphs $F$ in a certain set $\mathcal{A}$ of admissible graphs. It is not quite clear exactly which graphs should be admissible (see Subsection 3.5), so there are several variants of the definitions. To keep things simple, we shall work here with one particular choice for the set $\mathcal{A}$, depending on the function $p$. It may be that the various conjectures we shall make, if true, extend to larger sets $\mathcal{A}$.

Given a loopless multi-graph $F$ and an integer $t \geq 1$, let $F_{t}$ denote the graph obtained by subdividing each edge of $F$ exactly $t-1$ times, so $e\left(F_{t}\right)=t e(F)$ and $\left|F_{t}\right|=|F|+(t-1) e(F)$. Writing $\mathcal{F}^{\mathrm{m}}$ for the set of isomorphism classes of finite loopless multi-graphs, for $t \geq 2$ let

$$
\mathcal{F}_{t}=\left\{F_{t}: F \in \mathcal{F}^{\mathrm{m}}\right\},
$$

and set $\mathcal{F}_{1}=\mathcal{F}\left(\right.$ not $\left.\mathcal{F}^{\mathrm{m}}\right)$. Thus, for $t \geq 2$, the family $\mathcal{F}_{t}$ is the set of simple graphs that may be obtained as follows: starting with a set of paths of length $t$, identify subsets of the endpoints of these paths in an arbitrary way, except that the two endpoints of the same path may not be identified. Note that any $F_{t} \in \mathcal{F}_{t}$ has girth at least $2 t$.

Similarly, let $\mathcal{F}_{\geq t}$ be the set of simple graphs that may be obtained as above but starting with paths of length at least $t$. Thus $\mathcal{F}_{\geq t}$ is the set of graphs that may be obtained from some $F \in \mathcal{F}^{\mathrm{m}}$ by subdividing each edge at least $t-1$ times. Note that $\mathcal{F}=\mathcal{F}_{\geq 1} \supset \mathcal{F}_{\geq 2} \supset \cdots$. Let $\mathcal{T}$ denote the set of (isomorphism classes of) finite trees. 
For the rest of this section we suppose that there is some $\varepsilon>0$ such that $n p \geq n^{\varepsilon}$ for all large enough $n$. Equivalently, there is some integer $t \geq 1$ such that $n^{t-1} p^{t} \geq n^{-o(1)}$. We shall set $\mathcal{A}=\mathcal{T} \cup \mathcal{F}_{\geq t}$ for the smallest such $t$, noting that if $p=n^{-o(1)}$ then $t=1$, so all graphs are admissible. (An alternative that would work just as well is to let $\mathcal{A}$ be the set of all subgraphs of graphs in $\mathcal{F}_{\geq t}$, which includes $\mathcal{T}$.) On the one hand, this set $\mathcal{A}$ is small enough to satisfy the requirements for admissibility discussed in Subsection 3.5 (although there may be requirements we have missed, in which case $\mathcal{A}=\mathcal{T} \cup \mathcal{F}_{\geq t}$ for some larger $t$ is likely to work). On the other hand, as we shall now see, the set is large enough to ensure that the counts for $F \in \mathcal{A}$ determine a kernel, up to the equivalence relation $\sim$ defined in Subsection 2.4

Theorem 5.1. Let $\kappa_{1}$ and $\kappa_{2}$ be two bounded kernels, and $t \geq 1$ an odd integer. Suppose that $s\left(F, \kappa_{1}\right)=s\left(F, \kappa_{2}\right)$ for every $F \in \mathcal{F}_{t}$. Then $\kappa_{1} \sim \kappa_{2}$.

Proof. Given a kernel $\kappa$, let $\kappa^{t}$ be the kernel defined by

$$
\kappa^{t}(x, y)=\int_{[0,1]^{t-1}} \kappa\left(x, x_{1}\right) \kappa\left(x_{1}, x_{2}\right) \cdots \kappa\left(x_{t-1}, y\right) d x_{1} \cdots d x_{t-1}
$$

In other words, roughly speaking, $\kappa^{t}(x, y)$ counts the number of paths from $x$ to $y$ in $\kappa$ with length $t$. The key observation is that if $F$ is a graph, $\kappa$ a kernel, and $t \geq 1$, then

$$
s\left(F_{t}, \kappa\right)=s\left(F, \kappa^{t}\right)
$$

Indeed, $s\left(F_{t}, \kappa\right)$ is defined as an integral over one variable for each vertex of $F_{t}$. We may evaluate this integral by first fixing the variables corresponding to vertices of $F$, then using (31) once for each edge of $F$ to integrate over the remaining variables. What remains is exactly the integral defining $s\left(F, \kappa^{t}\right)$.

By assumption, $s\left(F, \kappa_{1}\right)=s\left(F, \kappa_{2}\right)$ for every $F \in \mathcal{F}_{t}$. Hence, from (32), we have $s\left(F, \kappa_{1}^{t}\right)=s\left(F, \kappa_{2}^{t}\right)$ for every graph $F$, so, by Theorem 2.6, $\kappa_{1}^{t} \sim \kappa_{2}^{t}$. Hence, from (10), there is a kernel $\kappa$ and measure-preserving maps $\sigma_{1}, \sigma_{2}:[0,1] \rightarrow[0,1]$ such that $\left(\kappa_{i}^{t}\right)^{\left(\sigma_{i}\right)}=\kappa$ a.e., for $i=1,2$. Since $\left(\kappa_{i}^{\left(\sigma_{i}\right)}\right)^{t}=\left(\kappa_{i}^{t}\right)^{\left(\sigma_{i}\right)}$, we thus have $\left(\kappa_{1}^{\prime}\right)^{t}=\left(\kappa_{2}^{\prime}\right)^{t}$ a.e. for $\kappa_{i}^{\prime}=\kappa_{i}^{\left(\sigma_{i}\right)}$. Since $\kappa_{i}^{\prime} \sim \kappa_{i}$, and our aim is to prove that $\kappa_{1} \sim \kappa_{2}$, it suffices to prove that $\kappa_{1}^{\prime} \sim \kappa_{2}^{\prime}$. Hence, without loss of generality, we may replace $\kappa_{i}$ by $\kappa_{i}^{\prime}$, so we have $\kappa_{1}^{t}=\kappa_{2}^{t}$ almost everywhere. It is now a matter of simple analysis to deduce that $\kappa_{1}=\kappa_{2}$ a.e.

Given a bounded signed kernel, i.e., a bounded function $\kappa:[0,1]^{2} \rightarrow \mathbb{R}$ satisfying $\kappa(x, y)=\kappa(y, x)$, let $T_{\kappa}$ be the corresponding operator on $L^{2}([0,1])$, defined by

$$
\left(T_{\kappa} f\right)(x)=\int_{0}^{1} \kappa(x, y) f(y) d y
$$


From the Cauchy-Schwartz inequality we have

$$
\begin{aligned}
\left\|T_{\kappa} f\right\|_{2}^{2} & =\int_{0}^{1}\left(\int_{0}^{1} \kappa(x, y) f(y) d y\right)^{2} d x \\
& \leq \int_{0}^{1}\left(\int_{0}^{1} \kappa(x, y)^{2} d y \int_{0}^{1} f(y)^{2} d y\right) d x \\
& =\|f\|_{2}^{2} \iint \kappa(x, y)^{2} d x d y=\|f\|_{2}^{2}\|\kappa\|_{2}^{2},
\end{aligned}
$$

so the operator norm of $T_{\kappa}$ on $L^{2}$ satisfies

$$
\left\|T_{\kappa}\right\| \leq\|\kappa\|_{2}<\infty
$$

Now let $\kappa$ be any bounded kernel, and $\varepsilon$ a positive real number. By Lemma 4.8 there is some $k$ such that $\left\|\kappa-\kappa_{k}\right\|_{2} \leq \varepsilon$. Writing $T_{\kappa}=T_{\kappa_{k}}+T_{\kappa-\kappa_{k}}$, the first term has finite rank, since $T_{\kappa_{k}} f$ is constant on intervals of length $2^{-k}$. From (34), the second term has operator norm at most $\left\|\kappa-\kappa_{k}\right\|_{2} \leq \varepsilon$. It follows that the image of the unit ball under $T$ can be covered by a finite number of balls of radius $2 \varepsilon$. Since $\varepsilon$ was arbitrary, this shows that $T_{\kappa}$ is a compact operator.

Since $\kappa$ is symmetric, we have shown that $T_{\kappa}$ is a compact self-adjoint operator, for any kernel $\kappa$. As in the matrix case, it now follows that $t$ th roots are unique. Indeed, since $T_{\kappa_{1}}$ is a compact self-adjoint operator on the Hilbert space $L^{2}(0,1)$, by standard results (see, for example, Bollobás [8]) this Hilbert space has an orthonormal basis consisting of eigenvectors of $T_{\kappa_{1}}$, with all eigenvalues real. Since $T_{\kappa_{1}^{t}}=\left(T_{\kappa_{1}}\right)^{t}$, it follows that $T_{\kappa_{1}^{t}}$ has the same eigenspaces as $T_{\kappa_{1}}$. (It is here that we use $t$ odd; otherwise, the map $\lambda \mapsto \lambda^{t}$ is not injective.) Turning this around, the action of $T_{\kappa_{1}}$ on each eigenspace $E_{\lambda}$ of $T_{\kappa_{1}^{t}}$ with eigenvalue $\lambda$ is to multiply by $\lambda^{1 / t}$. Thus, $T_{\kappa_{1}}$ is uniquely determined by $T_{\kappa_{1}^{t}}$. In particular, since $\kappa_{1}^{t}=\kappa_{2}^{t}$ a.e., the operators $T_{\kappa_{1}}$ and $T_{\kappa_{2}}$ are equal, i.e., $\kappa_{1}=\kappa_{2}$ a.e., as required.

Note that in Theorem 5.1 the restriction to odd $t$ is essential, as shown by the following example.

Example 5.2. Let $\kappa_{1}$ and $\kappa_{2}$ be the two 2-by-2 'chessboard' kernels defined by

$$
\kappa_{1}(x, y)= \begin{cases}1 & \text { if } x<1 / 2, y<1 / 2 \text { or } x \geq 1 / 2, y \geq 1 / 2 \\ 0 & \text { otherwise }\end{cases}
$$

and

$$
\kappa_{2}(x, y)= \begin{cases}1 & \text { if } x<1 / 2, y \geq 1 / 2 \text { or } x \geq 1 / 2, y<1 / 2 \\ 0 & \text { otherwise }\end{cases}
$$

Thus, in the dense case, $\kappa_{1}$ corresponds to the union of two disjoint complete graphs on $n / 2$ vertices, and $\kappa_{2}$ to the complete $n / 2$-by- $n / 2$ bipartite graph. It is easy to check that for any graph $F$ we have $s\left(F, \kappa_{1}\right)=2^{1-|F|}$, while $s\left(F, \kappa_{2}\right)=2^{1-|F|}$ if $F$ is bipartite, and $s\left(F, \kappa_{2}\right)=0$ otherwise. In particular, $s\left(F, \kappa_{1}\right)=s\left(F, \kappa_{2}\right)$ for all bipartite $F$, and hence for all $F \in \mathcal{F}_{t}, t$ even. 
As we saw from Lemma 4.2 and Corollary 4.7 , bounded density is a natural condition to impose on our sequence $\left(G_{n}\right)$ when dealing with $d_{\text {cut }}$ for sparse graphs. In the previous sections, when dealing with subgraph counts and $d_{\text {sub }}$, we imposed different conditions, the closest being Assumption 3.2. Let us restate this here in the appropriate form when $p$ need not be as large as $n^{-o(1)}$.

Assumption 5.3 (exponentially bounded admissible subgraph counts). There is a constant $C$ such that, for each fixed $F \in \mathcal{A}$, we have $\limsup s_{p}\left(F, G_{n}\right) \leq$ $C^{e(F)}$ as $n \rightarrow \infty$.

Note that we impose a condition only for $F \in \mathcal{A}$. When comparing $d_{\text {cut }}$ and $d_{\text {sub }}$, we need to impose both Assumptions 4.1 (bounded density) and 5.3 In the 'almost dense' case, when we take $\mathcal{A}=\mathcal{F}$, then Assumption 5.3 implies Assumption 4.1. with the same constant $C$. The argument is based on showing that a not-too-small dense part of $G_{n}$ would contain too many $K_{t, t}$ s for some large $t$. Since the details are very similar to the proof of Lemma 3.5, we omit them.

Unfortunately, in general neither of Assumptions 4.1 and 5.3 implies the other. In one direction, this is easy to see: simply add a complete graph on $m$ vertices, for some suitable $m(n)=o(n)$. This does not affect Assumption 4.1 but, if $m$ is chosen large enough, will create too many copies of any fixed graph $F$. For the reverse direction, consider the following example.

Example 5.4. Fix a real number $D>1$, and let $\kappa=\kappa_{D}$ be the unbounded kernel defined as follows. First partition $[0,1]$ into intervals $I_{1}, I_{2}, \ldots$, so that $I_{i}$ has length $2^{-i}$. Then set $\kappa(x, y)=i^{2 / D}$ if $x, y \in I_{i}$, and $\kappa(x, y)=0$ otherwise. Let $F$ be a connected graph with average degree at most $D$. Then

$$
s(F, \kappa)=\sum_{i=1}^{\infty} 2^{-i|F|} i^{2 e(F) / D} \leq \sum_{i=1}^{\infty}\left(2^{-i} i\right)^{|F|} \leq \sum_{i=1}^{\infty} 2^{-i} i=2 .
$$

Let $G_{n}=G_{p}(n, \kappa)$ be the random graph defined from $\kappa$ as before. If every component of any admissible graph has average degree at most $D$, then it is easy to check that with probability 1 the sequence $\left(G_{n}\right)$ satisfies Assumption 5.3 (with $C=2$ ). On the other hand, this sequence does not satisfy assumption $\mathrm{C}$, as there will be a subgraph of $G_{n}$ containing a positive fraction of the vertices with density around $i^{2 / D}$, for every $i$.

With the choice of $\mathcal{A}$ made here, whenever $p=p(n)$ does not satisfy $p=$ $n^{-o(1)}$ then only trees and graphs in some $\mathcal{F}_{\geq t}, t \geq 2$, are admissible. All such graphs, and all their components, have average degree less than 4 , so the example above shows that in this case, Assumption 5.3 does not imply Assumption 4.1.

Example 5.4 also shows that, in contrast to the almost dense case (where all graphs are admissible), in general we cannot tell from the admissible subgraph counts whether a (potential limiting) kernel is bounded. For this reason, together with those discussed above, when comparing $d_{\text {cut }}$ and $d_{\text {sub }}$ we impose both Assumptions 4.1 and 5.3 . 


\subsection{Conjectured equivalence between cut and count con- vergence}

Our main conjecture from Section 3 was that, in the sparse case, if the subgraph counts converge, they converge to those of a kernel. In the present setting, we mean counts for admissible subgraphs. Fix $p(n)$ as above, and a set $\mathcal{A}$ of admissible graphs. Throughout we impose Assumptions 4.1 and 5.3 for some fixed constant $C$. Let $X=[0, \infty)^{\mathcal{A}}$, let $s_{p}: \mathcal{F} \rightarrow X$ be the map defined by

$$
s_{p}\left(G_{n}\right)=\left(s_{p}\left(F, G_{n}\right)\right)_{F \in \mathcal{A}}
$$

for any graph $G_{n}$ with $n$ vertices, and let $d$ be a metric on $X$ inducing product topology, so $d_{\text {sub }}$ is defined by mapping to $X$ and then applying $d$; as usual, we suppress the dependence on the normalizing function $p$. Note that $d_{\text {sub }}$ is in general a pseudo-metric rather than a metric: there may be non-isomorphic graphs $G, G^{\prime}$ with $s_{p}(F, G)=s_{p}\left(F, G^{\prime}\right)$ for all $F \in \mathcal{A}$. As we only consider questions of convergence for sequences $G_{n}$ with $\left|G_{n}\right| \rightarrow \infty$, this will not be relevant.

Let $\mathcal{L} \subset X$ denote the set of possible limit points of sequences $s_{p}\left(G_{n}\right)$, where $\left(G_{n}\right)$ satisfies our assumptions.

Recall that we write $\mathcal{K}$ for the space of kernels, that is, symmetric measurable functions $\kappa:[0,1]^{2} \rightarrow[0, C]$ quotiented by equivalence. There is a natural map from $\mathcal{K}$ into $X$ given by subgraph counts; we write $s$ for this map, which does not depend on $p$ (except through the choice of $\mathcal{A}$ ). Since $\mathcal{A}$ always contains some set $\mathcal{F}_{\geq t}$, and hence some $\mathcal{F}_{t^{\prime}}$ with $t^{\prime}$ odd, Theorem 5.1 tells us that this map is injective.

In the present notation, our main conjecture, Conjecture 3.3, states exactly that every point of $\mathcal{L}$ arises in this way, i.e., that

$$
\mathcal{L} \subset s(\mathcal{K})
$$

We believe that the notions of convergence given by $d_{\text {sub }}$ and $d_{\text {cut }}$ are equivalent. The most concrete way of saying this is as follows.

Conjecture 5.5. Let $\left(G_{n}\right)$ be a sequence satisfying Assumptions 4.1 and 5.3, and let $\kappa \in \mathcal{K}$. Then $d_{\text {cut }}\left(G_{n}, \kappa\right) \rightarrow 0$ if and only if $s_{p}\left(G_{n}\right) \rightarrow s(\kappa)$.

In this form, the conjecture implies (35) (see below). Without assuming (35), it still makes sense to compare the notions of Cauchy sequences instead.

Conjecture 5.6. Let $\left(G_{n}\right)$ be a sequence satisfying Assumptions 4.1 and 5.3. Then $\left(G_{n}\right)$ is Cauchy with respect to $d_{\text {cut }}$ if and only if $\left(G_{n}\right)$ is Cauchy with respect to $d_{\mathrm{sub}}$.

As we shall shortly see, Conjectures 5.5 and 5.6 are equivalent.

Although we cannot prove the conjectures above, we can say something. Conjecture 5.5. for example, asserts two implications. Surprisingly, it is easy to show that, if (35) holds, then either of these implications (for all sequences, not 
just a particular sequence) implies the other! To prove this we shall first show that the random graph $G(n, \kappa)$ behaves 'correctly' with respect to our definition of $d_{\text {sub }}$; the corresponding result for $d_{\text {cut }}$ is Lemma 4.10 .

Lemma 5.7. Fix $C>0$, let $\kappa:[0,1]^{2} \rightarrow[0, C]$ be a bounded kernel, and let $G_{n}=G_{p}(n, \kappa)$. Then, with probability 1 , the sequence $\left(G_{n}\right)$ satisfies Assumption 5.3 and we have $s_{p}\left(G_{n}\right) \rightarrow s(\kappa)$.

Proof. The first statement follows from the second, since $s(F, \kappa) \leq C^{e(F)}$ holds for every $F$, and in particular for $F \in \mathcal{A}$.

It is well known that if $F$ is a fixed graph, and $p^{\prime}=p^{\prime}(n)$ is a function of $n$, then the number $X_{F}$ of subgraphs of $G\left(n, p^{\prime}\right)$ isomorphic to $F$ is concentrated about its mean if and only if $\mathbb{E}\left(X_{F^{\prime}}\right) \rightarrow \infty$ for every subgraph $F^{\prime}$ of $F$. Our choice of the set $\mathcal{A}$ ensures that this holds for every $F \in \mathcal{F}$ with $p^{\prime}=C p$, proving the result if $\kappa$ is constant. It is straightforward to adapt this result to finite type $\kappa$. It is easy to check that for the $F$ we consider, any $o\left(n^{2} p\right)$ edges of $G_{n} \subset G(n, C p)$ meet $o\left(n^{|F|} p^{e(F)}\right)$ copies of $F$. Using this observation, one can approximate the general case by the finite type case as in the proof of Lemma 4.10. We omit the details.

Lemma 5.7 gives us a sequence tending in $d_{\text {sub }}$ to any $\kappa \in \mathcal{K}$. In other words, it shows that $\mathcal{L} \supset s(\mathcal{K})$. Hence, if 35 holds,

$$
\mathcal{L}=s(\mathcal{K})
$$

Let $\mathcal{J} \subset \mathcal{K} \times \mathcal{L}$ denote the set of pairs $(\kappa, \lambda) \in \mathcal{K} \times \mathcal{L}$ such that there is a sequence $\left(G_{n}\right)$ satisfying our assumptions with $d_{\text {cut }}\left(G_{n}, \kappa\right) \rightarrow 0$ and $s_{p}\left(G_{n}\right) \rightarrow \lambda$. Together, Lemmas 4.10 and Lemma 5.7 tell us much more than simply that $\mathcal{L} \subset s(\mathcal{K})$ : they show that the 'diagonal' $\mathcal{D}=\{(\kappa, s(\kappa)): \kappa \in \mathcal{K}\}$ is contained in $\mathcal{J}$.

At this point, we have established three basic facts:

FACT 1: Every subsequence of $\left(G_{n}\right)$ has a subsequence converging in $d_{\text {sub }}$ to some point of $\mathcal{L}$. This is trivial, since Assumption 5.3 ensures that $s_{p}\left(G_{n}\right)$ lives in a compact subset of $X$.

FACT 2: Every subsequence of $\left(G_{n}\right)$ has a subsequence converging in $d_{\text {cut }}$ to some kernel $\kappa \in \mathcal{K}$. This is the first part of Corollary 4.7.

FACT 3: The map $s$ is an injection from $\mathcal{K}$ to $\mathcal{L}$. As noted above, this follows from Theorem 5.1 .

Facts 1 and 2 tell us that the relationship between the notions of convergence in $d_{\text {cut }}$ and $d_{\text {sub }}$ are described by the set $\mathcal{J}$. Indeed, any subsequence of $\left(G_{n}\right)$ itself has a subsequence in which we have convergence in both these metrics, to some point of $\mathcal{J}$.

Suppose for the moment that (36) holds. There are two possibilities.

If $\mathcal{J}$ is precisely the diagonal $\mathcal{D}$, then the three facts above easily imply that Conjectures 5.5 and 5.6 both hold.

If $\mathcal{J} \neq \mathcal{D}$, then there is some off diagonal point $\left(\kappa_{1}, \lambda\right)$ in $\mathcal{J}$. Since we are assuming (36), we have $\lambda=s\left(\kappa_{2}\right)$ for some $\kappa_{2} \in \mathcal{K}$. From the definition of 
$\mathcal{J}$ there is a sequence $\left(G_{n}\right)$ satisfying our assumptions, with $d_{\text {cut }}\left(G_{n}, \kappa_{1}\right) \rightarrow 0$ and $s_{p}\left(G_{n}\right) \rightarrow s\left(\kappa_{2}\right)$. Interleaving the sequence $G_{n}$ with the sequence $G_{p}(n, \kappa)$, which converges to $\kappa$ in both $d_{\text {cut }}$ and $d_{\text {sub }}$, taking $\kappa=\kappa_{1}$ or $\kappa_{2}$, we find a sequence which converges in one of $d_{\text {cut }}$ or $d_{\text {sub }}$ but not in the other. Hence, neither implication in Conjecture 5.5 or 5.6 holds, i.e., these conjectures fail as badly as possible.

In the light of the comments above, Conjecture 5.5 has the following rather vague reformulation as a question.

Question 5.8. Given a definition of 'suitable' sequences $\left(G_{n}\right)$, let $\mathcal{C}$ be the set of graphs such that, whenever $\kappa$ is a bounded kernel and $\left(G_{n}\right)$ is a suitable sequence with $d_{\text {cut }}\left(G_{n}, \kappa\right) \rightarrow 0$, then $s_{p}\left(F, G_{n}\right) \rightarrow s(F, \kappa)$. Under what reasonable definition of 'suitable' is the set $\mathcal{C}$ large enough that the counts $s(F, \kappa), F \in \mathcal{C}$, determine a kernel $\kappa$ up to equivalence?

The point is that, if $\mathcal{C}$ is large enough, then the three facts above hold with $\mathcal{A}=\mathcal{C}$, and we simply use $\mathcal{C}$ as the set of subgraph counts we use to define $d_{\text {sub }}$. Then, for our 'suitable' sequences, $d_{\text {cut }}$ convergence implies $d_{\text {sub }}$ convergence to the same kernel by definition, so $(\kappa, \lambda) \in \mathcal{J}$ implies $\lambda=s(\kappa)$. Thus (35) (and hence (36)) holds, and $\mathcal{J}=\mathcal{D}$, so $d_{\text {sub }}$ convergence also implies $d_{\text {cut }}$ convergence. Unfortunately, there is no obvious single choice for the set of suitable sequences. One could hope that sequences with bounded density would do, but this is not the case: by adding a complete graph with many (but still $o\left(p n^{2}\right)$ ) edges to $G(n, p)$, say, it is easy to check that in this case $\mathcal{C}$ consists only of matchings. Conjecture 5.5 is more specific than Question 5.8 since we define 'suitable' by assuming $s_{p}\left(F, G_{n}\right)$ bounded for $F$ in some set $\mathcal{A}$, and then require $\mathcal{C} \supset \mathcal{A}$.

If (35) (our main conjecture) does not hold, then Conjectures 5.5 and 5.6 cannot hold. Indeed, there is some $\lambda \in \mathcal{L}$ not corresponding to a kernel. Taking $G_{n}$ converging to $\lambda$ in $d_{\text {sub }}$, and then a subsequence that converges in $d_{\text {cut }}$, there is some $\kappa$ with $(\kappa, \lambda) \in \mathcal{J}$. Interleaving a corresponding sequence $\left(G_{n}\right)$ with $G_{p}(n, \kappa)$, we find a sequence that converges in $d_{\text {cut }}$ but not in $d_{\text {sub }}$.

Even if (35) does not hold, it is still possible that there is some relationship between cut and subgraph convergence: it may be that every sequence that is Cauchy with respect to $d_{\text {sub }}$, and hence converges to some $\lambda \in \mathcal{L}$, is Cauchy with respect to $d_{\text {cut }}$, i.e., converges to some $\kappa \in \mathcal{K}$. This happens if and only if, for every $\lambda \in \mathcal{L}$, there is a unique $\kappa \in \mathcal{K}$ such that $(\kappa, \lambda) \in \mathcal{J}$. This is not as implausible as it may sound. Indeed, suppose Conjecture 5.5 holds for some admissible set $\mathcal{A}_{-}$, but that the definitions involved make sense for a larger set $\mathcal{A}_{+}$. It may be that (35) fails working with $\mathcal{A}_{+}$, because we are now allowing as admissible some counts which need not converge to what we expect. However, there is a restriction map from $\mathcal{L}_{+}$to $\mathcal{L}_{-}$forgetting about the counts outside $\mathcal{A}_{-}$. Since (36) holds for the smaller set of admissible graphs, this would show that for the larger set there is only one $\kappa$ for each $\lambda$, but not vice versa.

In the next section we shall prove a form of Conjecture 5.5 Before doing so, let us briefly compare this conjecture with the corresponding result of Borgs, 
Chayes, Lovász, Sós and Vesztergombi [17] for the dense case. In the dense setting, Facts 1 and 2 above are easy to prove. That all limiting counts come from kernels was shown by Lovász and Szegedy [35]; this gives (36). Surprisingly, the hard part is proving Fact 3, that the counts (now meaning all counts)

determine the kernel, up to equivalence as defined in Subsection 2.4. (For us this was easy, since we deduced the sparse equivalent of this statement from the dense result, Theorem 2.6) Once one knows that the counts determine the kernel, the 'meta-argument' above shows that $d_{\text {cut }}$ convergence implies $d_{\text {sub }}$ convergence if and only if the reverse implication holds. Since the forward implication is very easy, the result of 17 that the two metrics are equivalent follows. However, the only way we know to show that the counts determine the kernel is to use this result of Borgs, Chayes, Lovász, Sós and Vesztergombi [17, which is not proved this way!

\subsection{Partial results: embedding lemmas}

Our aim in this section is to prove a positive result, that under certain circumstances, if $d_{\text {cut }}\left(G_{n}, \kappa\right) \rightarrow 0$, then $s_{p}\left(F, G_{n}\right) \rightarrow s_{p}(F, \kappa)$ for certain graphs $F$. In the case where $\kappa$ is of finite type, this is simply a counting lemma: in this case, $G_{n} \rightarrow \kappa$ says that $G_{n}$ can be partitioned into $(\varepsilon, p)$-regular pairs with densities given by $\kappa$. In the uniform case, Chung and Graham [19] proved such counting lemmas for certain graphs under certain assumptions. The general case turns out to be rather different, but we shall still use several of their ideas.

We start with the simplest case, where $F$ is a path. First we need some definitions. As usual, in the proof it will be easier to consider homomorphisms from $F$ to $G_{n}$ (i.e., walks in $G_{n}$ ) rather than embeddings. As we shall see later, this makes no difference.

For $G_{n}$ a graph and $X_{0}, \ldots, X_{\ell}$ subsets of $V\left(G_{n}\right)$, let $G_{n}\left(X_{0}, X_{1}, \ldots, X_{\ell}\right)$ denote the number of $(\ell+1)$-tuples $\left(v_{i}\right)$ with $v_{i} \in X_{i}$ and $v_{i} v_{i+1} \in E(G)$ for $0 \leq i \leq \ell-1$. (We could just as well consider the number of paths with $v_{i} \in X_{i}$, but the formulae are slightly cleaner with walks.) Identifying a subset of $V\left(G_{n}\right)$ with a subset of $[0,1]$ as before, for a kernel $\kappa$ let

$$
\kappa\left(X_{0}, X_{1}, \ldots, X_{\ell}\right)=\int_{X_{0} \times \cdots \times X_{\ell}} \kappa\left(x_{0}, x_{1}\right) \cdots \kappa\left(x_{\ell-1}, x_{\ell}\right) d x_{0} \cdots d x_{\ell} .
$$

Lemma 5.9. Let $C>0$ be constant, let $p(n)$ be any function of $n$ with $n p \rightarrow \infty$, and let $\left(G_{n}\right)$ be a sequence of graphs with $t_{p}\left(T, G_{n}\right)$ bounded for each tree $T$. For every $\varepsilon>0$ and $\ell \geq 1$ there is a $\delta=\delta_{\ell}(\varepsilon)>0$ such that, whenever $\kappa:[0,1]^{2} \rightarrow[0, C]$ is a kernel with $\left\|\kappa_{G_{n}}-\kappa\right\|_{\text {cut }} \leq \delta$, then

$$
\left|G_{n}\left(X_{0}, X_{1}, \ldots, X_{\ell}\right)-n^{\ell+1} p^{\ell} \kappa\left(X_{0}, X_{1}, \ldots, X_{\ell}\right)\right| \leq \varepsilon n^{\ell+1} p^{\ell}
$$

for any sets $X_{0}, X_{1} \ldots, X_{\ell} \subset V\left(G_{n}\right)$.

Roughly speaking, the lemma says that if $G_{n} \rightarrow \kappa$ and $t_{p}\left(T, G_{n}\right)$ is bounded for each $T$, then $t_{p}\left(P_{\ell}, \kappa\right) \rightarrow s\left(P_{\ell}, \kappa\right)$. The stronger assertion makes it simpler to prove the result by induction. 
Proof. Renormalizing, we may assume without loss of generality that $C=1$. Let us do so from now on.

The fact that $\delta$ is not allowed to depend on $\kappa$ allows us to assume without loss of generality that $\kappa$ is piecewise constant on squares of side $1 / n$, i.e., that $\kappa$ may be interpreted as a (dense) weighted graph with vertex set $V\left(G_{n}\right)$. Indeed, the Frieze-Kannan form of Szemerédi's Lemma shows that there is an integer $k$ such that, given any $\kappa$, there is a $\kappa^{\prime}$ that is constant on squares of side $1 / k$ with $d_{\text {cut }}\left(\kappa, \kappa^{\prime}\right) \leq \delta$. Tweaking $\kappa^{\prime}$ slightly if $k$ does not divide $n$, we obtain a kernel $\kappa^{\prime \prime}$ of the required form. Replacing $\delta$ by $2 \delta$ as appropriate, the result for $\kappa$ follows from the result for $\kappa^{\prime \prime}$. [Note that we implicitly assumed that $n$ is large here, meaning larger than some $n_{0}$ depending on $\varepsilon$ and $\ell$. We could simply assume this in the statement of the lemma, but in it can be achieved by subdividing vertices. In fact, we could work with a kernel instead of a graph throughout the proof.]

Let

$$
\Delta\left(X_{0}, \ldots, X_{\ell}\right)=\frac{G_{n}\left(X_{0}, X_{1}, \ldots, X_{\ell}\right)}{n^{\ell+1} p^{\ell}}-\kappa\left(X_{0}, X_{1}, \ldots, X_{\ell}\right)
$$

so our aim is to show that $\left|\Delta\left(X_{0}, \ldots, X_{\ell}\right)\right| \leq \varepsilon$ for all choices of the sets $X_{i}$. We shall show much more: let $M=\max _{T} \sup _{n} t_{p}\left(T, G_{n}\right)$, where the maximum is over trees with at most $2 \ell+1$ vertices, noting that $M<\infty$. We shall show that if $d_{\text {cut }}\left(G_{n}, \kappa\right) \leq \delta$, then, for any $1 \leq t \leq \ell$ and any $X_{0}, \ldots, X_{t} \subset V\left(G_{n}\right)$ we have

$$
\left|\Delta\left(X_{0}, X_{1}, \ldots, X_{t}\right)\right| \leq \varepsilon_{t}
$$

where $\varepsilon_{1}=\delta$, and

$$
\varepsilon_{t}=7 \sqrt{\varepsilon_{t-1}}+\sqrt{M} \varepsilon_{t-1}^{1 / 4}
$$

for $t \geq 2$. Since $\varepsilon_{\ell}$ tends to zero as $\delta \rightarrow 0$, this implies the result.

We proceed by induction on $t$. For $t=1$, the result is immediate from the definition of the cut metric: indeed, $\Delta\left(X_{0}, X_{1}\right)$ is one of the quantities appearing in the supremum defining this metric. Suppose now that $2 \leq t \leq \ell$, and that (37) holds with $t$ replaced by $t-1$.

For $v \in V(G)$ and $X_{1}, \ldots, X_{r} \subset V(G)$, set

$$
\kappa\left(v, X_{1}, \ldots, X_{r}\right)=\int_{X_{1} \times \cdots \times X_{r}} \kappa\left(x, x_{1}\right) \kappa\left(x_{1}, x_{2}\right) \cdots \kappa\left(x_{r-1}, x_{r}\right) d x_{1} \cdots d x_{r}
$$

where $x$ is any point of the interval of length $1 / n$ corresponding to the vertex $v$, and let

$$
\Delta\left(v, X_{1}, \ldots, X_{r}\right)=\frac{G_{n}\left(\{v\}, X_{1}, \ldots, X_{r}\right)}{n^{r} p^{r}}-\kappa\left(v, X_{1}, \ldots, X_{r}\right) .
$$

Note that

$$
\Delta\left(X, X_{1}, \ldots, X_{r}\right)=\frac{1}{n} \sum_{v \in V} \Delta\left(v, X_{1}, \ldots, X_{r}\right)
$$


Fix $X_{0}, \ldots, X_{t} \subset V\left(G_{n}\right)$, and set $\varepsilon=\sqrt{\varepsilon_{t-1}}$. Let $B_{1}$ be the set of $v \in X_{1}$ with $\Delta\left(v, X_{2}, \ldots, X_{t}\right)>\varepsilon$. Then, from (39), $\Delta\left(B_{1}, X_{2}, \ldots, X_{t}\right) \geq \varepsilon\left|B_{1}\right| / n$. But by induction, $\Delta\left(B_{1}, X_{2}, \ldots, X_{t}\right) \leq \varepsilon_{t-1}=\varepsilon^{2}$. Hence, $\left|B_{1}\right| \leq \varepsilon n$. Arguing similarly, and using $\varepsilon_{t-1} \geq \varepsilon_{1}$, we see that the set $B$ of vertices $v \in X_{1}$ for which either $\left|\Delta\left(v, X_{2}, \ldots, X_{t}\right)\right| \geq \varepsilon$ or $\left|\Delta\left(v, X_{0}\right)\right| \geq \varepsilon$ has size at most $4 \varepsilon$.

If $v \in X_{1} \backslash B$, then we have roughly the right number of walks through $v$, i.e.,

$$
G_{n}\left(X_{0},\{v\}, X_{2}, \ldots, X_{t}\right)=G_{n}\left(\{v\}, X_{0}\right) G_{n}\left(\{v\}, X_{2}, \ldots, X_{t}\right)
$$

is close to $n p \kappa\left(v, X_{0}\right) n^{t-1} p^{t-1} \kappa\left(v, X_{2}, \ldots, X_{t}\right)$. More precisely, using the fact that $\kappa$ is pointwise bounded by $C=1$ to bound the $\kappa$ terms in the last expression by 1 , for $v \in X_{1} \backslash B$ we have

$$
\left|\Delta\left(X_{0}, v, X_{2}, \ldots, X_{t}\right)\right| \leq 3 \varepsilon,
$$

where the left hand side is defined by analogy with (38).

It remains to consider $v \in B$. For $i=1,2$, let

$$
\sigma_{i}=\sum_{v \in B} G_{n}\left(X_{0},\{v\}, X_{2}, \ldots, X_{t}\right)^{i},
$$

noting that $\sigma_{1} \leq \sqrt{|B| \sigma_{2}}$ by the Cauchy-Schwartz inequality. Let $T$ be the tree with $2 t$ edges formed by identifying the second vertices of two paths of length $t$. Then $\sigma_{2}$ counts a subset of the homomorphisms from $T$ into $G_{n}$, so

$$
\sigma_{2} \leq \operatorname{hom}\left(T, G_{n}\right)=n^{2 t+1} p^{2 t} t_{p}\left(T, G_{n}\right) \leq M n^{2 t+1} p^{2 t} .
$$

Since $|B| \leq 4 \varepsilon n$ it follows that

$$
\sigma_{1} \leq \sqrt{|B| \sigma_{2}} \leq 2 \sqrt{M \varepsilon} n^{t+1} p^{t} .
$$

Since $\kappa$ is bounded by 1 , we have $\kappa\left(X_{0}, B, X_{2}, \ldots, X_{t}\right) \leq \mu(B) \leq 4 \varepsilon$, so

$$
\left|\Delta\left(X_{0}, B, X_{2}, \ldots, X_{t}\right)\right| \leq 2 \sqrt{M \varepsilon}+4 \varepsilon .
$$

Together with the bound (40) for $v \in X_{1} \backslash B$, this implies that

$$
\left|\Delta\left(X_{0}, X_{1}, \ldots, X_{t}\right)\right| \leq 7 \varepsilon+2 \sqrt{M \varepsilon}=\varepsilon_{t},
$$

as required. This completes the proof of (37) by induction, and thus the proof of the lemma.

Note that the argument above works just as well for an arbitrary fixed tree rather than a path: we pick some leaf $v$ to play the role of $x_{0}$; the unique neighbour of $v$ then plays the role of $x_{1}$. This gives us a counting lemma for trees.

Corollary 5.10. Let $\left(G_{n}\right)$ be a sequence of graphs with $t_{p}\left(T, G_{n}\right)$ bounded for every tree $T$, and suppose that $d_{\text {cut }}\left(G_{n}, \kappa\right) \rightarrow 0$, where $\kappa$ is a bounded kernel. Then for each tree $T$ we have $t_{p}\left(T, G_{n}\right) \rightarrow s\left(\kappa, G_{n}\right)$ as $n \rightarrow \infty$. 
Chung and Graham [19] proved a version of this result (for paths rather than trees) with $\kappa$ constant, under the assumption that the maximum degree of $G_{n}$ is at most $C p n$. This maximum degree assumption of course gives $t_{p}\left(T, G_{n}\right) \leq$ $C^{e(T)}$, so it is stronger than the bounded tree counts assumption of Lemma 5.9 In some sense, the maximum degree condition is much stronger, but it turns out that our global assumption is just as good for questions involving subgraph counts. The reason that Lemma 5.9 is more complicated than the corresponding simple result in [19] is that $\kappa$ is not uniform, not our weaker assumption.

We stated earlier that, in the sparse case, the parameter $s_{p}(F, \kappa)$ should be preferred to $t_{p}(F, \kappa)$, even though $t_{p}$ tends to be easier to work with. Nevertheless, in the case of trees, these parameters are equivalent, as shown by the following observation.

Lemma 5.11. Let $p(n)$ be any function of $n$ with $n p \rightarrow \infty$, and let $\left(G_{n}\right)$ be a sequence with $s_{p}\left(T, G_{n}\right)$ bounded for every tree $T$. Then, for each tree $T$, we have $t_{p}\left(T, G_{n}\right) \sim s_{p}\left(T, G_{n}\right)$. In particular, $t_{p}\left(T, G_{n}\right)$ is bounded.

Proof. Fix a tree $T$ with $k$ vertices. It suffices to show that the number $N_{T}$ of non-injective homomorphisms from $T$ to $G_{n}$ satisfies $N_{T}=o\left(n^{k} p^{k-1}\right)$ as $n \rightarrow \infty$. Now the image of any non-injective homomorphism $\phi$ from $T$ to $G_{n}$ is a connected subgraph $H$ of $G_{n}$ with $\ell$ vertices, where $1 \leq \ell \leq k-1$. Any such subgraph contains a tree $T^{\prime}$ with $\ell$ vertices, so for each $\ell$ there are (crudely) at most $\sum_{\left|T^{\prime}\right|=\ell} \operatorname{emb}\left(T^{\prime}, G_{n}\right)$ possibilities for vertex set of $H$, where the sum is over all trees $T^{\prime}$ with $\ell$ vertices. Since there are at most $k^{\ell}$ homomorphisms $\phi$ with image a given set of $\ell$ vertices, we thus have

$$
N_{T} \leq \sum_{\ell=1}^{k-1} k^{\ell} \sum_{\left|T^{\prime}\right|=\ell} \operatorname{emb}\left(T^{\prime}, G_{n}\right) .
$$

Since $\operatorname{emb}\left(T^{\prime}, G_{n}\right)=n_{\left(|T|^{\prime}\right)} p^{e\left(T^{\prime}\right)} s_{p}\left(T^{\prime}, G_{n}\right)$, the final term is $O\left(n^{\ell} p^{\ell-1}\right)$ by assumption. It follows that $N_{T}=O\left(n^{k-1} p^{k-2}\right)=o\left(n^{k} p^{k-1}\right)$, as claimed.

Lemma 5.11 allows us to restate Corollary 5.10 in terms of the parameter $s$.

Theorem 5.12. Let $\left(G_{n}\right)$ be a sequence of graphs with $s_{p}\left(T, G_{n}\right)$ bounded for every tree $T$, and suppose that $d_{\text {cut }}\left(G_{n}, \kappa\right) \rightarrow 0$, where $\kappa$ is a bounded kernel. Then for each tree $T$ we have $s_{p}\left(T, G_{n}\right) \rightarrow s\left(\kappa, G_{n}\right)$ as $n \rightarrow \infty$.

Theorem 5.12 may be regarded as an embedding lemma for trees. Our next aim is to prove a much more general result. Chung and Graham showed that, in the uniform case, if the number of paths of length $\ell-1$ between any two vertices is at most a constant times what it should be, then almost all pairs of vertices are joined by almost the right number of paths of length $\ell$, and hence $G_{n}$ contains asymptotically the expected number of copies of any $F \in \mathcal{F}_{\ell}$. This result is much harder than the paths result, even in the uniform case. Although we shall use the key idea of Chung and Graham, the proof does not carry over in a simple way. In the following result, we work with $t_{p}$ rather than $s_{p}$ for simplicity; we return to this later. 
Theorem 5.13. Let $C>0$ and $\ell \geq 2$ be fixed, and let $p=p(n)$ be any function of $n$. Let $\left(G_{n}\right)$ be a sequence of graphs with $\sup _{n} t_{p}\left(F, G_{n}\right)<\infty$ for each $F \in \mathcal{T} \cup \mathcal{F}_{\ell} \cup\left\{C_{2 \ell-2}\right\}$, and suppose that $d_{\text {cut }}\left(G_{n}, \kappa\right) \rightarrow 0$ for some kernel $\kappa:[0,1]^{2} \rightarrow[0, C]$. Then $t_{p}\left(F, G_{n}\right) \rightarrow s(F, \kappa)$ for each $F \in \mathcal{F}_{\ell}$.

Proof. Note that by Lemma 4.2 the sequence $\left(G_{n}\right)$ has density bounded by $C$, i.e., it satisfies Assumption 4.1

Fix $\varepsilon>0$, and a graph $F_{\ell} \in \mathcal{F}_{\ell}$. Let $\eta>0$ be a small constant to be chosen below (depending on $\varepsilon, \ell$ and $F_{\ell}$ ). For $n$ large enough, which we assume from now on, let $P_{1}, \ldots, P_{k}$ be an $(\eta, p)$-regular partition of $G_{n}$, where $k$ does not depend on $n$. Such a partition exists by Lemma 4.5. As usual, we shall ignore rounding to integers, assuming that each $P_{i}$ contains exactly $n / k$ vertices. Let $\kappa^{\prime}$ be the kernel taking the value $d_{p}\left(P_{i}, P_{j}\right)$ on the set $P_{i} \times P_{j} \subset[0,1]^{2}$. Note that $\kappa^{\prime}$ is bounded by $C+1$ if $n$ is large enough, which we assume. Note also that $d_{\text {cut }}\left(\kappa, \kappa^{\prime}\right) \leq d_{\text {cut }}\left(G_{n}, \kappa\right)+d_{\text {cut }}\left(G_{n}, \kappa^{\prime}\right) \leq 2 \varepsilon$ if we choose $\eta$ small enough (for the second term) and then $n$ large enough. Hence, for any fixed $F$,

$$
\left|s(F, \kappa)-s\left(F, \kappa^{\prime}\right)\right| \leq O(\varepsilon),
$$

and it suffices to show that $t_{p}\left(F_{\ell}, G_{n}\right)$ is close to $s\left(F_{\ell}, \kappa^{\prime}\right)$ rather than to $s\left(F_{\ell}, \kappa\right)$. To avoid clutter in the notation, from now on we write $\kappa$ for the kernel $\kappa^{\prime}$ associated to our $(\eta, p)$-regular partition: the original $\kappa$ plays no further role in the proof. Recall that $\kappa$ (formerly known as $\kappa^{\prime}$ ) is bounded by $C+1$. At this point we shall renormalize, multiplying $p(n)$ by $C+1$ and dividing $\kappa$ by $C+1$. After doing so, $\kappa$ is bounded by 1 .

For $v, w \in V\left(G_{n}\right)$ and $t \geq 1$, let $w_{t}(v, w)$ denote the number of walks of length $t$ in $G_{n}$ starting at $v$ and ending at $w$; we suppress the dependence on $G_{n}$ in the notation. Let $\kappa^{t}(v, w)$ denote the normalized 'expected' number of such walks, if $G_{n}$ behaved like the random graph $G_{p}(n, \kappa)$. Let $U \subset V^{2}$ be the set of pairs $(v, w)$ such that $w_{\ell}(v, w) \leq\left(\kappa^{\ell}(v, w)-\varepsilon\right) n^{\ell-1} p^{\ell}$. We call the pairs $(v, w) \in U$ underconnected, since they are joined by 'too few' walks of length $\ell$. We shall show that

$$
|U|=\left|\left\{(v, w): w_{\ell}(v, w) \leq\left(\kappa^{\ell}(v, w)-\varepsilon\right) n^{\ell-1} p^{\ell}\right\}\right| \leq \varepsilon n^{2}
$$

if $\eta$ is chosen suitably, and then $n$ is taken large enough. Before doing so, let us note that this implies the result.

By Lemma [5.9] if we choose $\eta$ small enough, then the total number of walks of length $\ell$ in $G_{n}$ is within $\varepsilon n^{\ell+1} p^{\ell}$ of the expected number in $G_{p}(n, \kappa)$, namely $\left\|\kappa^{\ell}\right\|_{1} n^{\ell+1} p^{\ell}$. If (41) holds, then if we count only a maximum of $\kappa^{\ell}(v, w) n^{\ell-1} p^{\ell}$ walks for each pair $(v, w)$ of endpoints, we still count at least $(1-\varepsilon)\left(\left\|\kappa^{\ell}\right\|_{1}-\right.$ $\varepsilon) n^{\ell+1} p^{\ell}$ walks, so there are at most $3 \varepsilon n^{\ell+1} p^{\ell}$ walks uncounted, using $\left\|\kappa^{\ell}\right\|_{1} \leq 1$. Writing $W$ for the set of overconnected pairs $(v, w) \in V^{2}$ with $w_{\ell}(v, w) \geq$ $\left(\kappa^{\ell}(v, w)+\sqrt{\varepsilon}\right) n^{\ell-1} p^{\ell}$, it follows that

$$
|W| \leq 3 \sqrt{\varepsilon} .
$$

In other words, almost all pairs of vertices are joined by almost the right number of walks. 
Recall that we fixed a graph $F_{\ell} \in \mathcal{F}_{\ell}$. Let $F_{\ell}$ be obtained by subdividing the edges of a loopless multi-graph $F$ with vertex set $u_{1}, \ldots, u_{r}$, so

$$
\operatorname{hom}\left(F_{\ell}, G_{n}\right)=\sum_{v_{1}, \ldots, v_{r} \in V\left(G_{n}\right)} \prod_{u_{i} u_{j} \in E(F)} w_{\ell}\left(v_{i}, v_{j}\right),
$$

where the factors in the product corresponding to multiple edges of $F_{\ell}$ are of course repeated. Given $u_{i} u_{j} \in E(F)$, let $2 F_{\ell} / E_{2}$ be the graph formed from two copies of $F_{\ell}$ by identifying the vertices corresponding to $u_{i}$ and identifying the vertices corresponding to $u_{j}$. Since $2 F_{\ell} / E_{2} \in \mathcal{F}_{\ell}$, we have $t_{p}\left(2 F_{\ell} / E_{2}, G_{n}\right)$ bounded. It follows by the Cauchy-Schwartz inequality that the number of homomorphisms from $F_{\ell}$ into $G_{n}$ mapping $u_{i}$ and $u_{j}$ to a pair in $U \cup W$ is small, in fact of order $\varepsilon^{1 / 4} n^{\left|F_{\ell}\right|} p^{e\left(F_{\ell}\right)}$; the argument is as in the proof of Lemma 5.9.

Since the comment above applies to any edge $u_{i} u_{j}$ of $F$, the contribution to the sum in (43) from terms in which one or more pairs $\left(v_{i}, v_{j}\right)$ fall in $U \cup$ $W$ is small. But in the remaining terms, $w_{\ell}\left(v_{i}, v_{j}\right)$ is well approximated by $\kappa^{\ell}\left(v_{i}, v_{j}\right) n^{\ell-1} p^{\ell}$, and it follows that $t_{p}\left(F_{\ell}, G_{n}\right)$ is close to $s\left(F_{\ell}, \kappa\right)$ : the difference is bounded by some function of $|F|$ and $\varepsilon$. In short, we have shown that to prove the theorem, it suffices to prove (41), i.e., that there are few underconnected pairs.

From now on, we forget the original graph $F$, and aim to prove (41). It will be convenient to assume that $\varepsilon$ is fairly small. In particular, we shall assume that $\varepsilon \leq 1 / 40$.

Recall that all but at most $\eta k^{2}$ pairs in our partition $\left(P_{i}\right)_{1}^{k}$ are $(\eta, p)$-regular. Since all pairs have density at most $C$, the irregular pairs contain at most $C \eta n^{2} p$ edges. Since $t_{p}\left(T, G_{n}\right)$ is bounded for each tree $T$, and in particular for the trees formed from two paths by identifying an edge from each, by Cauchy-Schwartz again a small set of edges meets only a small fraction of the walks of length $\ell$ in $G_{n}$. In particular, the number of walks of length $\ell$ containing one or more edges from irregular pairs is $O\left(\sqrt{\eta} n^{\ell+1} p^{\ell}\right)$. Taking $\eta$ small enough, we may assume that this quantity is less than $\varepsilon^{2} n^{\ell+1} p^{\ell} / 10$, say. It follows that in proving (41), we may delete all edges in irregular pairs, i.e., we may assume that every pair is regular: if (41) holds for the resulting graph $G_{n}^{\prime}$ and kernel $\kappa^{\prime}$ with $\varepsilon / 2$ in place of $\varepsilon$, then (41) holds for our original graph $G_{n}$ and kernel $\kappa$.

The lower bound in the proof of Lemma 5.9 used only closeness of the graph and kernel in the cut norm, not the bounds on various tree counts. This argument can thus be applied locally to sequences of parts of our partition. Abusing notation, let us write $P_{0}, P_{1}, \ldots, P_{\ell-1}$ for an arbitrary sequence of $\ell$ parts of our partition, with repetition allowed. For any subsets $X_{i} \subset P_{i}$, we find that there are at least

$$
\prod_{i=0}^{\ell-1}\left|X_{i}\right| p^{\ell-1} \prod_{i=0}^{\ell-2} \kappa\left(P_{i}, P_{i+1}\right)-\gamma(n / k)^{\ell} p^{\ell-1}
$$

walks $v_{0} v_{1} \cdots v_{\ell-1}$ with $v_{i} \in X_{i}$, where $\gamma=\gamma(\eta, \ell)$ tends to 0 as $\eta \rightarrow 0$. We choose $\eta$ small enough that $\gamma \leq \varepsilon^{12}$. Taking $X_{i}=P_{i}$ for $i>0$, and summing over all choices for the intermediate parts, a consequence of this is that if $P_{0}$ 
and $P_{\ell-1}$ are any two parts, and $X_{0}$ any subset of $P_{0}$, then there are at least

$$
\left(\kappa^{\ell-1}(x, y)\left|X_{0}\right| /\left|P_{0}\right|-\gamma\right) n^{\ell} p^{\ell-1} / k^{2}
$$

walks of length $\ell-1$ from $X_{0}$ to $P_{\ell-1}$, where $x \in P_{0}$ and $y \in P_{\ell-1}$.

Let us call a walk of length $\ell-1$ in $G_{n}$ bad if there are at least $M n^{\ell-2} p^{\ell-1}$ walks in $G_{n}$ with the same endpoints, where $M$ is a constant to be chosen in a moment, depending on $\varepsilon$ but not on $\eta$; otherwise, the walk is good. Each bad walk may be extended to at least $M n^{\ell-2} p^{\ell-1}$ homomorphic images of $C_{2 \ell-2}$. By assumption, $t_{p}\left(C_{2 \ell-2}, G_{n}\right)$ is bounded, so it follows that there are $O\left(n^{\ell} p^{\ell-1} / M\right)$ bad walks. In particular, choosing the constant $M$ large enough, we may assume that there are at most $\varepsilon^{9} n^{\ell} p^{\ell-1} / 3$ bad walks.

Suppose for a contradiction that (41) does not hold, i.e., the set $U$ of underconnected pairs of vertices has size at least $\varepsilon n^{2}$. Our first aim is to select a pair $\left(P, P^{\prime}\right)$ of parts of our partition such that there are many underconnected pairs $(u, v)$ in $P \times P^{\prime}$, but not too many bad walks start in $P$. Since $|U| \geq \varepsilon n^{2}$ by assumption, there are at least $\varepsilon k / 2$ parts $P$ with

$$
|U \cap(P \times V)| \geq \varepsilon n^{2} /(2 k) .
$$

On the other hand, there are at most $\varepsilon k / 3$ parts $P$ with the property that more than $\varepsilon^{8} n^{\ell} p^{\ell-1} / k$ bad walks start in $P$ (otherwise there would be too many bad walks). Hence there exists a part $P$ for which (45) holds, with at most $\varepsilon^{8} n^{\ell} p^{\ell-1} / k$ bad walks starting in $P$. Pick such a $P$. From (45) and averaging, there is a part $P^{\prime}$ such that

$$
\left|U \cap\left(P \times P^{\prime}\right)\right| \geq \varepsilon n^{2} /\left(2 k^{2}\right)=\varepsilon|P|\left|P^{\prime}\right| / 2 .
$$

From now on, fix such a $P^{\prime}$.

Let us say that a pair $\left(u, P^{\prime \prime}\right)$ with $u \in P$ and $P^{\prime \prime}$ a part of our partition is deficient if there are fewer than $\left(\kappa^{\ell-1}(x, y)-\sqrt{\gamma}\right) n^{\ell-1} p^{\ell-1} / k$ walks of length $\ell-1$ from $u$ to $P^{\prime \prime}$, where $x \in P$ and $y \in P^{\prime \prime}$, and $\gamma$ is as in (44). For a given $P^{\prime \prime}$, at most $\sqrt{\gamma} n / k$ vertices $u \in P$ form a deficient pair with $P^{\prime \prime}$ : otherwise, the set $X_{0}$ of such vertices would have more than $\gamma n^{\ell} p^{\ell-1} / k^{2}$ fewer walks to $P^{\prime \prime}$ that it should have, contradicting (44). Hence, there are at most $\sqrt{\gamma} n$ deficient pairs. Let $D \subset P$ be the set of vertices $u$ in more than $\gamma^{1 / 4} k$ deficient pairs. Then $|D| \leq \sqrt{\gamma} n /\left(\gamma^{1 / 4} k\right)=\gamma^{1 / 4}|P|$.

Let us say that a pair $\left(u, P^{\prime \prime}\right)$ with $u \in P$ and $P^{\prime \prime}$ a part of our partition is compromised if there are more than $\varepsilon^{3} n^{\ell-1} p^{\ell-1} / k$ bad walks from $u$ to $P^{\prime \prime}$. Since at most $\varepsilon^{8} n^{\ell} p^{\ell-1} / k$ bad walks start in $P$, there are at most $\varepsilon^{5} n$ compromised pairs. Let $C$ be the set of $u \in P$ in more than $\varepsilon^{3} k$ compromised pairs; then $|C| \leq \varepsilon^{2} n / k=\varepsilon^{2}|P|$.

Let $S \subset P$ be the set of vertices $u$ for which there are at least $\varepsilon\left|P^{\prime}\right| / 4$ vertices $v \in P^{\prime}$ with $(u, v) \in U$. By (46) we have

$$
\varepsilon|P|\left|P^{\prime}\right| / 2 \leq\left|U \cap\left(P \times P^{\prime}\right)\right| \leq|S|\left|P^{\prime}\right|+\varepsilon|P|\left|P^{\prime}\right| / 4,
$$


so $|S| \geq \varepsilon|P| / 4>\left(\gamma^{1 / 4}+\varepsilon^{2}\right)|P|$. Thus $|S|>|D|+|C|$, and there is some $u$ in $S \backslash(D \cup C)$. Fix such a $u$ for the rest of the proof. Let $U_{u}$ denote the set of $v \in P^{\prime}$ for which $(u, v)$ is underconnected.

At this point we have chosen a vertex $u$, a part $P^{\prime}$, and a set $U_{u} \subset P^{\prime}$ with the following properties:

(i) for each $v \in U_{u}$, there are at most $\left(\kappa^{\ell}(u, v)-\varepsilon\right) n^{\ell-1} p^{\ell}$ walks of length $\ell$ from $u$ to $v$.

(ii) $\left|U_{u}\right| \geq \varepsilon\left|P^{\prime}\right| / 4$,

(iii) there are at most $\gamma^{1 / 4} k \leq \varepsilon^{3} k$ deficient pairs $\left(u, P^{\prime \prime}\right)$,

(iv) there are at most $\varepsilon^{3} k$ compromised pairs $\left(u, P^{\prime \prime}\right)$.

From (i) and (ii) above, there are at least $m=\varepsilon^{2} n^{\ell} p^{\ell} /(4 k)$ 'missing walks' from $u$ to $U_{u}$ : the number of walks of length $\ell$ from $u$ to $U_{u}$ falls short of the expected number in $G_{p}(n, \kappa)$ by at least $m$. Let $P^{\prime \prime}$ be any part of our partition. By a $u-U_{u}$ walk via $P^{\prime \prime}$ we mean a walk of length $\ell$ from $u$ to $U_{u}$ whose second last vertex lies in $P^{\prime \prime}$; the expected number of such walks is $N_{P^{\prime \prime}}=$ $\kappa^{\ell-1}(u, w) \kappa(w, v)\left|U_{u}\right| n^{\ell-1} p^{\ell} / k$, where $w$ is any point of $P^{\prime \prime}$ and $v$ any point of $P^{\prime} \supset U_{u}$. Note that $\sum_{P^{\prime \prime}} N_{P^{\prime \prime}}$ is simply the expected number of walks from $u$ to $U_{u}$. Let $m_{P \text { " }}$ be the number of 'missing walks via $P^{\prime \prime}$, , i.e., the difference between $N_{P^{\prime \prime}}$ and the number of $u-U_{u}$ walks via $P^{\prime \prime}$, or zero if there are at least $N_{P^{\prime \prime}}$ such walks. The total number of missing walks is at most the sum of the numbers $m_{P^{\prime \prime}}$, so

$$
\sum_{P^{\prime \prime}} m_{P^{\prime \prime}} \geq m \geq \varepsilon^{2} n^{\ell} p^{\ell} /(4 k)
$$

Let us say that $P^{\prime \prime}$ is useful if $m_{P^{\prime \prime}} \geq \varepsilon^{2} n^{\ell} p^{\ell} /\left(8 k^{2}\right)$, so the contribution to the sum above from non-useful parts $P^{\prime \prime}$ is at most half the right hand side. Recalling that we have normalized so that $\kappa$ is bounded by 1 , For each $P^{\prime \prime}$ we have $m_{P^{\prime \prime}} \leq N_{P^{\prime \prime}} \leq n^{\ell} p^{\ell} / k^{2}$; it follows that there are at least $\varepsilon^{2} k / 8 \geq 5 \varepsilon^{3} k$ useful parts $P^{\prime \prime}$.

Using (iii) and (iv) above, it follows that there is a part $P^{\prime \prime}$ which is useful, but neither deficient nor compromised. Fix such a part $P^{\prime \prime}$.

Recall that a walk of length $\ell-1$ from $u$ to $w \in P^{\prime \prime}$ is good if it is not bad, i.e., if

$$
w_{\ell-1}(u, w) \leq N=M n^{\ell-2} p^{\ell-1} .
$$

Since $\gamma^{1 / 4} \leq \varepsilon^{3}$, and $P^{\prime \prime}$ is neither deficient nor compromised, there are at least

$$
\left(\kappa^{\ell-1}(u, w)-2 \varepsilon^{3}\right) n^{\ell-1} p^{\ell-1} / k
$$

good walks from $u$ to $P^{\prime \prime}$. On the other hand, there are many missing walks via $P^{\prime \prime}$. With this setup, we are finally ready to apply the key idea of Chung and Graham [19, which is to partition the set $P^{\prime \prime}$ into subsets according to the approximate number of walks from $u$ to the relevant vertex, and then use regularity to show that there are about the right number of walks from $U_{u}$ to each such subset. In fact, there is a slick way of doing this. 


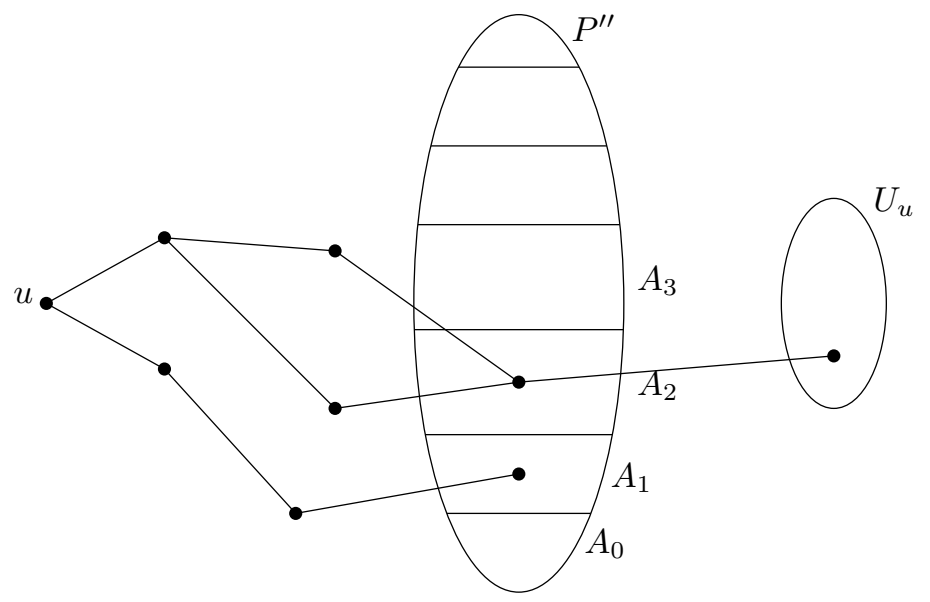

Figure 1: The set $P^{\prime \prime}$ is subdived into sets $A_{i}$, with $w_{\ell-1}(u, v)=i$ for each $v \in A_{i}$. Each edge from $A_{i}$ to $U_{u}$ contributes $i$ walks from $u$ to $U_{u}$ via $P^{\prime \prime}$.

For $i \geq 0$, let $A_{i}$ be the set of vertices $v \in P^{\prime \prime}$ with $w_{\ell-1}(u, v)=i$; see Figure 1. Also, let $A_{i}^{+}=\bigcup_{j \geq i} A_{i}$. Then,

$$
w_{\ell-1}\left(u, P^{\prime \prime}\right)=\sum_{i \geq 0} i\left|A_{i}\right|=\sum_{i \geq 1}\left|A_{i}^{+}\right| .
$$

More importantly, $\sum_{i=1}^{N}\left|A_{i}^{+}\right|$is at least the number of good walks from $u$ to $P^{\prime \prime}$, so

$$
\sum_{i=1}^{N}\left|A_{i}^{+}\right| \geq\left(\kappa^{\ell-1}(u, w)-2 \varepsilon^{3}\right) n^{\ell-1} p^{\ell-1} / k .
$$

Since $\left(P^{\prime \prime}, P^{\prime}\right)$ is $(\eta, p)$-regular with (normalized) density $\kappa(w, v) \leq 1$, if $A \subset P^{\prime \prime}$ and $B \subset P^{\prime}$ then $e(A, B) \geq p \kappa(w, v)|A||B|-\eta p(n / k)^{2}$ (this is trivially true if one of $A$ or $B$ has size less than $\eta n / k)$. Since each edge from $U_{u}$ to $A_{i}$ forms the final edge of exactly $i$ walks from $u$ to $U_{u}$, the number of walks from $u$ to $U_{u}$ via $P^{\prime \prime}$ is given by

$$
\begin{aligned}
\sum_{i \geq 1} i e\left(A_{i}, U_{u}\right) & \geq \sum_{i=1}^{N} e\left(A_{i}^{+}, U_{u}\right) \\
& \geq \sum_{i=1}^{N} p \kappa(w, v)\left|A_{i}^{+}\right|\left|U_{u}\right|-\eta p(n / k)^{2} \\
& \geq\left(\kappa^{\ell-1}(u, w)-2 \varepsilon^{3}\right) \kappa(w, v)\left|U_{u}\right| n^{\ell-1} p^{\ell} / k-\eta N p(n / k)^{2}
\end{aligned}
$$

where we used (47) in the last step. The main term is simply the expected number of walks from $u$ to $U_{u}$ via $P^{\prime \prime}$, so the conclusion is that there are at most

$$
2 \varepsilon^{3} \kappa(w, v)\left|U_{u}\right| n^{\ell-1} p^{\ell} / k+\eta N p(n / k)^{2}
$$


missing walks from $u$ to $U_{u}$ via $P^{\prime \prime}$. The two terms above may be bounded above by $2 \varepsilon^{3} n^{\ell} p^{\ell} / k^{2}$ and $\eta M n^{\ell} p^{\ell} / k^{2}$, respectively. Choosing $\eta \leq \varepsilon^{3} / M$ we thus have at most $3 \varepsilon^{3} n^{\ell} p^{\ell} / k^{2}$ missing walks via $P^{\prime \prime}$, i.e., $m_{P^{\prime \prime}} \leq 3 \varepsilon^{3} n^{\ell} p^{\ell} / k^{2}$, which contradicts the fact that $P^{\prime \prime}$ is useful. This contradiction completes the proof.

The proof of Theorem 5.13 actually gives rather more with almost no extra work.

Theorem 5.14. Let $C>0$ and $\ell \geq 2$ be fixed, and let $p=p(n)$ be any function of $n$. Let $\left(G_{n}\right)$ be a sequence of graphs with $\sup _{n} t_{p}\left(F, G_{n}\right)<\infty$ for each $F \in \mathcal{T} \cup \mathcal{F}_{\geq \ell} \cup\left\{C_{2 \ell-2}\right\}$, and suppose that $d_{\text {cut }}\left(G_{n}, \kappa\right) \rightarrow 0$ for some bounded kernel $\kappa$. Then $t_{p}\left(F, G_{n}\right) \rightarrow s(F, \kappa)$ for each $F \in \mathcal{T} \cup \mathcal{F}_{\geq \ell}$.

Proof. The conclusion for $F \in \mathcal{T}$ follows from Corollary 5.10 ,

Fix $F \in \mathcal{F}_{\geq \ell}$ and $\varepsilon>0$, and let $L$ be the length of the longest induced path in $F$. Since the hypotheses of Theorem 5.13 are satisfied with $\ell$ replaced by any $t$ in the range $\ell \leq t \leq L$, the proof of that result shows that if $\eta$ is chosen small enough, then when we take an $(\eta, p)$-regular partition of $G_{n}$ with associated kernel $\kappa^{\prime}$, almost all pairs $(v, w)$ of vertices are joined almost the 'right' number of walks of each length $t, \ell \leq t \leq L$. More precisely, writing $\kappa$ for $\kappa^{\prime}$ as in the proof of Theorem 5.13 and writing $U_{t}$ for the set of pairs $(v, w)$ with $w_{t}(v, w) \leq\left(\kappa^{t}(v, w)-\varepsilon\right) n^{t-1} p^{t}$ and $W_{t}$ for the set of pairs with $w_{t}(v, w) \geq$ $\left(\kappa^{t}(v, w)+\sqrt{\varepsilon}\right) n^{t-1} p^{t}$, the proof of Theorem 5.13 shows that $\left|U_{t}\right| \leq \varepsilon n^{2}$ for $\ell \leq t \leq L$, and (hence) that $\left|W_{t}\right| \leq 2 \sqrt{\varepsilon} n^{2}$ for each $t$ in this range. Using the analogue of (43) in which each term $w_{\ell}(\cdot, \cdot)$ is replaced by an appropriate term $w_{t}(\cdot, \cdot)$, as before we can use the Cauchy-Schwartz inequality to show that the contribution to $t_{p}\left(F, G_{n}\right)$ from terms with some pair $\left(v_{i}, v_{j}\right)$ in the small set $\bigcup_{t} U_{t} \cup W_{t}$ is small (of order $\varepsilon^{1 / 4}$ ), and it follows as before that if $\eta$ is small enough, then $\left|t_{p}\left(F, G_{n}\right)-s(F, \kappa)\right|$ is bounded by some function of $F$ and $\varepsilon$, giving the result.

Let us note for later reference that, in one way, the assumptions of Theorems 5.13 and 5.14 are weaker than they may first appear. Let $F$ be a loopless multigraph with vertex set $u_{1}, u_{2}, \ldots, u_{k}$, and let $F_{\ell} \in \mathcal{F}_{\ell}$ be obtained by subdividing each edge of $F$ exactly $\ell-1$ times. Then (43) may be rewritten as

$$
\operatorname{hom}\left(F_{\ell}, G_{n}\right)=n^{k} \mathbb{E}\left(\prod_{u_{i} u_{j} \in E(F)} w_{\ell}\left(v_{i}, v_{j}\right)\right),
$$

where the expectation is over the uniform choice of $\left(v_{1}, v_{2}, \ldots, v_{k}\right) \in V\left(G_{n}\right)^{k}$. Applying Hölder's inequality, $\mathbb{E}\left(\prod_{i=1}^{r} X_{i}\right) \leq\left(\prod \mathbb{E}\left(\left|X_{i}\right|^{r}\right)\right)^{1 / r}$, with $r=e(F)$, it follows that

$$
\begin{aligned}
\operatorname{hom}\left(F_{\ell}, G_{n}\right)^{r} \leq n^{k r} \prod_{u_{i} u_{j} \in E(F)} \mathbb{E}\left(w_{\ell}\left(v_{i}, v_{j}\right)^{r}\right) & =n^{k r} \mathbb{E}\left(w_{\ell}\left(v_{1}, v_{2}\right)^{r}\right)^{r} \\
& =n^{k r-2 r} \operatorname{hom}\left(H_{r, \ell}, G_{n}\right)^{r},
\end{aligned}
$$


where $H_{r, \ell} \in \mathcal{F}_{\ell}$ is the 'theta-graph' consisting of $r$ internally vertex disjoint paths of length $\ell$ joining the same pair of vertices. The normalizing factors work out correctly, so we have

$$
t_{p}\left(F_{\ell}, G_{n}\right) \leq t_{p}\left(H_{r, \ell}, G_{n}\right) .
$$

Hence, the condition that $t_{p}\left(F, G_{n}\right)$ remain bounded for every $F \in F_{\ell}$ is equivalent to the condition that $t_{p}\left(F, G_{n}\right)$ is bounded for $F=H_{r, \ell}, r=1,2, \ldots$.

Arguing similarly, for any $F \in F_{\geq \ell}$ we may bound $t_{p}\left(F, G_{n}\right)$ in terms of the quantities $t_{p}\left(H_{r, \ell^{\prime}}, G_{n}\right)$, where $\ell^{\prime}$ ranges over the lengths of the paths making up $F$. Hence, to show that $t_{p}\left(F, G_{n}\right)$ is bounded for all $F \in F_{\geq \ell}$, it suffices to prove the same condition for the graphs $H_{r, \ell^{\prime}}, r \geq 1, \ell^{\prime} \geq \ell$. Note that these latter conditions are simply moment conditions on the numbers of walks of various lengths joining a random pair of vertices of $G_{n}$.

In the case where the limiting kernel $\kappa$ is of finite type, Theorem 5.14 may be seen as a form of counting lemma. In this case, it is easy to strengthen the result to count homomorphisms from $F$ into $G_{n}$ with each vertex mapped to a specified part of the partition of $G_{n}$ corresponding to the finite type kernel $\kappa$, obtaining a result similar in form to Lemma 5.9. Such a (strengthened) finite type case of Theorem 5.13 or Theorem 5.14 is very much easier to prove than the general case: there is no need to apply Szemerédi's Lemma, and the proof of the result of Chung and Graham [19] for the uniform case goes through without much modification. One might hope that, using Szemerédi's Lemma, the full generality of Theorem 5.14 would follow easily from the finite type case, but this is not true. The problem is that our assumptions are inescapably global: we assume, for instance, that the number of copies of $C_{2 \ell-2}$ in $G_{n}$ is bounded by a multiple of the expected number of copies. When we take an $(\varepsilon, p)$-regular partition, this gives no useful information about the number of copies of $C_{2 \ell-2}$ in each regular pair: we have a bound that is of the form $M k^{2 \ell-2}$ times the expected number of copies, where $k$ is the number of parts. To apply the finite type case, we would need a bound independent of $k$. For this reason there seems to be no easy way around the work in the proof of Theorem 5.13.

Theorem 5.14 may be seen as some progress towards a proof of some form of Conjecture 5.5. More precisely, it is almost an answer to Question 5.8 the only problem is that for Theorem 5.14 we work with $t_{p}$ rather than $s_{p}$. We shall return to this in detail in a moment. However, even ignoring this, Theorem 5.14 is a little disappointing in some ways. Let $\mathcal{A}=\mathcal{T} \cup \mathcal{F}_{\geq \ell}$. Assuming boundedness of $t_{p}\left(F, G_{n}\right)$ for $F \in \mathcal{A} \cup\left\{C_{2 \ell-2}\right\}$, we obtain convergence of the counts $t_{p}\left(F, G_{n}\right)$ for $F \in \mathcal{A}$. The extra assumption for $F=C_{2 \ell-2}$ is somehow annoying. This is perhaps clearest if we consider the range where $p$ is fairly large, say $n^{-o(1)}$. In this case $s_{p} \sim t_{p}$, and it makes sense to assume boundedness of all counts $s_{p}\left(F, G_{n}\right)$. However, since $C_{2}$ does not make sense, the smallest value of $\ell$ for which we can apply Theorem 5.14 is $\ell=3$, and we obtain convergence of the counts $s_{p}\left(F, G_{n}\right)$ for $F \in \mathcal{F}_{\geq 3}$. In comparison with Theorem 3.18, this is disappointing: the latter result shows us that with the counts $s_{p}$ bounded, and 
$s_{p}\left(C_{4}, G_{n}\right) \rightarrow 1$, which should roughly correspond to convergence to the uniform kernel $\kappa=1$, we obtain $s_{p}\left(F, G_{n}\right) \rightarrow s(F, \kappa)=1$ for all $F \in \mathcal{F}_{\geq 2}$, rather than just for $F \in \mathcal{F}_{\geq 3}$.

In fact, Theorem 3.18 gives much more: it gives convergence for all $F$ with girth at least 4. Chung and Graham [19] asked whether an analogous result holds for sparse graphs under the appropriate assumptions (what they call ' $\ell$-quasi randomness', which corresponds roughly to the assumptions of Theorem 5.13 with $\kappa$ constant), with girth at least 4 replaced by girth at least $2 \ell$. In our language, they asked whether the conclusion of Theorem 5.13 can be extended to all $F$ with girth at least $2 \ell$. Unfortunately, the answer is no for a trivial reason, namely that there are graphs with arbitrarily large girth and arbitrarily large average degree. Taking $p=n^{-\alpha}$ for some $0<\alpha<1$, and $d$ large enough, for any graph $F$ with average degree $d$ the expected number of copies of $F$ in $G(n, p)$ is $o(1)$, so the normalizing constant in the definition of $t_{p}\left(F, G_{n}\right)$ is $o(1)$. Since $\operatorname{hom}\left(F, G_{n}\right)$ is an integer, we cannot have $t_{p}\left(F, G_{n}\right) \rightarrow 1$ in this case.

\subsection{Embeddings or homomorphisms?}

In this subsection we return to the use of $t_{p}$ rather than $s_{p}$ in Theorems $\$ 5.13$ and 5.14. Although this simplifies the proof, it is unsatisfactory for a reason we shall now explain. We start by discussing the corresponding problem with the corresponding result of Chung and Graham [19, their Theorem 8. We shall use the following fact, proved by Blakley and Roy [6] in a slightly more general form in the context of symmetric matrices.

Theorem 5.15. Let $G$ be a graph with $n$ vertices and average degree $d$. Then $G$ contains at least $n d^{\ell}$ walks of length $\ell$.

Recall that we write $w_{t}(u, v)$ for the number of walks of length $t$ from $u$ to $v$. Chung and Graham [19 impose the condition that $w_{\ell-1}(u, v)<c_{0} p^{\ell-1} n^{\ell-2}$ holds for every pair of vertices $u, v$, where $c_{0}$ is a constant: they call this condition $U(\ell)$. In other words, the number of walks from $u$ to $v$ is at most a constant times what it should be. Normalizing so that $G_{n}$ contains exactly $p n^{2} / 2$ edges, Chung and Graham note that $U(\ell)$ can only hold if $p=\Omega\left(n^{-1+1 /(\ell-1)}\right)$ : otherwise, the expected number of walks of length $\ell-1$ from a random $u$ to a random $v$ is much less than 1 , so $w_{\ell-1}(u, v)$ must sometimes be much larger than its expectation.

In fact, $U(\ell)$ cannot hold unless $p$ is quite a bit larger, but for the 'wrong' reason: taking $\ell$ odd for simplicity, let $\ell=2 k+1$. Considering walks of length $\ell-1$ formed by tracing a walk of length $k$ forwards and then backwards, we see that if $G_{n}$ has $p n^{2} / 2$ edges, then

$$
\sum_{v} w_{\ell-1}(v, v) \geq \operatorname{hom}\left(P_{k}, G_{n}\right) \geq n(n p)^{k},
$$

where the second inequality is Theorem 5.15. Thus there is some $v$ with $w_{\ell-1}(v, v) \geq(n p)^{k}$, and it follows that $U(\ell)$ can only hold if $p=\Omega\left(n^{-1+2 /(\ell-1)}\right)$. 
It follows that Theorem 8 of [19] can only be applied in this range. Note that this is an essential problem: this result counts homomorphisms (Chung and Graham use the notation $\#\{H \subset G\}$ for $\operatorname{hom}(H, G))$, and the bound on $w_{\ell-1}(u, v)$ is definitely used with $u=v$. Indeed, as we shall see, the conclusion fails if $p=o\left(n^{-1+2 /(\ell-1)}\right)$.

Turning to Theorem 5.13 the condition that $t_{p}\left(C_{2 \ell-2}, G_{n}\right)$ remain bounded corresponds roughly to the condition $U(\ell)$ : indeed, the former says exactly that

$$
\sum_{u, v} w_{\ell-1}(u, v)^{2}=O\left(n^{2 \ell-2} p^{2 \ell-2}\right)
$$

which follows immediately from $U(\ell)$. It turns out that the problem described above does not arise with (50) - in this second moment (rather than uniform) condition, the few pairs with $u=v$ matter less. Indeed, it is easy to check that in $G(n, p)$, for example, (50) holds as long as $p=\Omega\left(n^{-1+1 /(\ell-1)}\right)$. [The expected number of homomorphisms from $C_{2 \ell-2}$ whose image is a tree with $k$ edges is $O\left(n(n p)^{k}\right)=O\left(n(n p)^{\ell-1}\right)$, and the expected number whose image is a graph with $k$ vertices containing a cycle is $O\left(n^{k} p^{k}\right)=O\left((n p)^{2 \ell-2}\right)$.] However, the same problem arises in a different place.

As before, let $H_{k, \ell} \in \mathcal{F}_{\ell}$ be the 'theta-graph' formed by $k$ paths of length $\ell$ joining the same pair $(s, t)$ of vertices, with the paths internally vertex disjoint. Suppose that $\ell$ is even. Writing $w_{t}(v)=w_{t}\left(v, V\left(G_{n}\right)\right)$ for the number of walks of length $t$ in $G_{n}$ starting at $v$, considering homomorphisms from $H_{k, \ell}$ to $G_{n}$ mapping $s$ and $t$ to a common vertex $v$, we have

$$
\operatorname{hom}\left(H_{k, \ell}, G_{n}\right) \geq \sum_{v} w_{\ell / 2}(v)^{k} \geq n\left(\frac{1}{n} \sum_{v} w_{\ell / 2}(v)\right)^{k} \geq n(n p)^{k \ell / 2},
$$

where the second inequality is from convexity and the last from Theorem 5.15 Since $\left|H_{k, \ell}\right|=2+k(\ell-1)$ and $e\left(H_{k, \ell}\right)=k \ell$, it follows that $t_{p}\left(H_{k, \ell}, G_{n}\right) \geq$ $n^{k-1}(n p)^{-k \ell / 2}$. Suppose that $p \leq n^{-1+2 / \ell-\varepsilon}$ for some $\varepsilon>0$. Then taking $k$ large enough we see that $t_{p}\left(H_{k, \ell}, G_{n}\right) \rightarrow \infty$, so neither the assumptions nor the conclusion of Theorem 5.13 can hold. This is much larger than the value of $p$ above which the number of subgraphs of $G(n, p)$ isomorphic to $H_{k, \ell}$ is well behaved.

The calculations above illustrate the problem with working with $t_{p}$ : we count certain trees as copies of $H_{k, \ell}$, for example, and the number of these trees exceeds the number of embeddings of $H_{k, \ell}$ in a wide range of densities in which Theorem 5.13 might otherwise apply. For this reason, if we could replace $t_{p}$ by $s_{p}$ throughout the statement of the theorem, we would obtain a much stronger and more satisfactory result: not only would it count embeddings, which is what we are really interested in, instead of homomorphisms, but it would apply to a much larger family of graphs, for example, to random graphs with much lower densities. Unfortunately, the proof breaks down in various places if we simply replace $t_{p}$ by $s_{p}$. However, the next result is a major step in this direction. 
Given vertices $v, w$ of a graph $G_{n}$, suppressing the dependence on $G_{n}$, let us write $p_{\ell}(v, w)$ for the number of paths of length $\ell$ from $v$ to $w$, so $p_{\ell}(v, w) \leq$ $w_{\ell}(v, w)$.

Theorem 5.16. Let $C>0$ and $\ell \geq 2$ be fixed, and let $p=p(n)$ be any function of $n$. Let $\left(G_{n}\right)$ be a sequence of graphs satisfying the following three conditions:

$$
\begin{aligned}
& \sup _{n} s_{p}\left(F, G_{n}\right)<\infty \text { for each } F \in \mathcal{T}, \\
& \sum_{u} \sum_{v \neq u} p_{\ell-1}(u, v)^{2}=O\left(n^{2 \ell-2} p^{2 \ell-2}\right),
\end{aligned}
$$

and

$$
\sum_{u} \sum_{v \neq u} p_{\ell}(u, v)^{k}=O\left(n^{2+k(\ell-1)} p^{k \ell}\right),
$$

for each fixed $k \geq 1$. Suppose also that $d_{\text {cut }}\left(G_{n}, \kappa\right) \rightarrow 0$ for some kernel $\kappa$ : $[0,1]^{2} \rightarrow[0, C]$. Then $s_{p}\left(F, G_{n}\right) \rightarrow s(F, \kappa)$ for each $F \in \mathcal{F}_{\ell}$.

Before turning to the proof of this result, let us make some remarks on the conditions above. Firstly, in (51) it makes no difference whether we write $s_{p}$ or $t_{p}$, by Lemma 5.11 .

Condition (53) is almost the same as the condition $s_{p}\left(H_{k, \ell}, G_{n}\right)=O(1)$. Indeed, $\operatorname{emb}\left(H_{k, \ell}, G_{n}\right)$ is simply the sum over distinct $u$ and $v$ of the number of $k$-tuples of internally vertex disjoint paths from $u$ to $v$, so (53), which bounds the same sum without the restriction to disjoint paths, is formally stronger than $s_{p}\left(H_{k, \ell}, G_{n}\right)=O(1)$. Since there are (typically) many paths from $u$ to $v$ in the range of $p$ for which (52) may hold, it seems very likely that, assuming the other conditions of Theorem 5.16, $s_{p}\left(H_{k, \ell}, G_{n}\right)=O(1)$ implies (53), so (53) could be replaced by this more pleasant condition. However, we do not have a proof of this.

Similarly, condition (52) is closely related to $s_{p}\left(C_{2 \ell-2}, G_{n}\right)=O(1)$, and could perhaps be replaced by this weaker condition. This is less clear, however, as Theorem 5.16] can be applied for $p$ small enough that the typical number of paths of length $\ell-1$ between a given pair of vertices is $O(1)$.

Instead of (52) we can always impose the stronger condition $t_{p}\left(C_{2 \ell-2}, G_{n}\right)=$ $O(1)$; these conditions are probably equivalent in the present setting. The corresponding statement for (53) and the stronger assumption $t_{p}\left(H_{k, \ell}, G_{n}\right)=O(1)$ is not true; see the extensive discussion earlier in the subsection.

Finally, let us note that (53) gives us control over $s_{p}\left(F_{\ell}, G_{n}\right)$ for all $F_{\ell} \in \mathcal{F}_{\ell}$, not just for $F_{\ell}=H_{k, \ell}$. Let $F_{\ell}$ be obtained by subdividing a graph $F$ with vertex set $u_{1}, u_{2}, \ldots, u_{k}$. Then

$$
\operatorname{emb}\left(F_{\ell}, G_{n}\right) \leq \sum_{v_{1}, v_{2}, \ldots, v_{k}} \prod_{u_{i} u_{j} \in E(F)} p_{\ell}\left(v_{i}, v_{j}\right)
$$

where the sum is over all $n_{(k)} k$-tuples of distinct vertices of $G_{n}$. Applying Hölder's inequality as in the proof (48) of (49), but in a probability space with 
$n_{(k)}$ elements rather than $n^{k}$, we find that

$$
\operatorname{emb}\left(F_{\ell}, G_{n}\right) \leq n_{(k)} \mathbb{E}\left(p_{\ell}\left(v_{1}, v_{2}\right)^{e(F)}\right),
$$

where the expectation is over the choice of a random pair $\left(v_{1}, v_{2}\right)$ of distinct vertices of $G_{n}$. Condition (53) bounds the final expectation; as usual the normalizing factors work out, and we see that if (53) holds for every $k$ then $s_{p}\left(F_{\ell}, G_{n}\right)=O(1)$ for every $F_{\ell} \in \mathcal{F}_{\ell}$.

Proof of Theorem [5.16. Since the proof is a relatively simple modification of that of Theorem 5.13. we shall give only an outline, concentrating on the differences.

The first change we make is that we work with paths rather than walks, replacing the quantities $w_{t}(u, v), t=\ell-1, \ell$, appearing in the proof of Theorem 5.13 with the corresponding quantities $p_{t}(u, v)$. By Lemma 5.11, all but a vanishing fraction of the walks in $G_{n}$ of a given length are paths, so (41), for example, implies the same statement with $w_{\ell}(v, w)$ replaced by $p_{\ell}(v, w)$. Of course, (41) was proved using the assumption $t_{p}\left(C_{2 \ell-2}, G_{n}\right)=O(1)$, whereas we now have the weaker assumption (52). However, following through the proof it is easy to see that if we count paths instead of walks, then (52) suffices. (The key point is that (52) suffices to bound the number of bad paths, i.e., paths between endpoints $u, v$ with $p_{\ell-1}(u, v)>M n^{\ell-2} p^{\ell-1}$.)

Let us fix $\varepsilon>0$ and a graph $F_{\ell} \in \mathcal{F}_{\ell}$. We also fix an integer $N$ to be chosen later, depending only on $\varepsilon$ and $F_{\ell}$. Finally, let $\eta$ be a small positive constant depending on $\varepsilon, F_{\ell}$ and $N$. For reasons that will become clear later, we first partition $V\left(G_{n}\right)$ into $N$ almost equal parts $Q_{1}, \ldots, Q_{N}$. Then we take an $(\eta, p)$ regular partition $\left(P_{i}\right)$ with each $P_{i}$ contained in some $Q_{j}$. For the moment we ignore the partition $\left(Q_{i}\right)$.

Let $\kappa$ be the kernel corresponding to our $(\eta, p)$-regular partition, and let $S \subset V \times V$ be the set of pairs of vertices joined by the 'wrong' number of paths of length $\ell$ :

$$
S=\left\{(v, w): v \neq w,\left|p_{\ell}(v, w)-\kappa^{\ell}(v, w)\right| \geq \varepsilon n^{\ell-1} p^{\ell}\right\} .
$$

If $\eta$ is chosen small enough then the proofs of (41) and (42) carry though counting paths instead of walks, and (replacing $\varepsilon$ by $\varepsilon^{2} / 10$ ), the equivalents of (411) and (42) imply that

$$
|S| \leq \varepsilon n(n-1)
$$

We proceed from here to our bound on $s_{p}\left(F_{\ell}, G_{n}\right)$ in two steps. First we count something that is not quite an embedding of $F_{\ell}$.

Let $F_{\ell}$ be obtained by subdividing each edge of the loopless multigraph $F$ $\ell-1$ times, and let $u_{1}, \ldots, u_{k}$ be the vertices of $F$, which we also regard as vertices of $F_{\ell}$. By a semiembedding of $F_{\ell}$ into $G_{n}$ we mean a homomorphism from $F_{\ell}$ into $G_{n}$ that maps the vertices $u_{1}, \ldots, u_{k}$ to distinct vertices of $G_{n}$, and each of the $e(F) u_{i}-u_{j}$ paths of length $\ell$ that make up the graph $F_{\ell}$ into a path in $G_{n}$. Clearly, every embedding is a semiembedding; the only additional condition on an embedding is that the paths in $G_{n}$ are internally vertex disjoint. 
Let $\mathrm{emb}^{+}\left(F_{\ell}, G_{n}\right) \geq \operatorname{emb}\left(F_{\ell}, G_{n}\right)$ denote the number of semiembeddings of $F_{\ell}$ into $G_{n}$. Then, from the definition of a semiembedding we have

$$
\mathrm{emb}^{+}\left(F_{\ell}, G_{n}\right)=\sum_{v_{1}, \ldots, v_{k}} \prod_{u_{i} u_{j} \in E(F)} p_{\ell}\left(v_{i}, v_{j}\right),
$$

where the sum is over all $n_{(k)}$ sequences $\left(v_{1}, \ldots, v_{k}\right)$ of distinct vertices of $G_{n}$ and, as usual, any multiple edges in $F$ give rise to multiple factors in the product.

As before we, we can rewrite the formula above as an expectation over a random choice of $\left(v_{1}, \ldots, v_{k}\right)$. Normalizing correctly for a change, let $X_{i j}$ be the random variable $p_{\ell}\left(v_{i}, v_{j}\right) /\left(n^{\ell-1} p^{\ell}\right)$, so

$$
s_{p}^{+}\left(F_{\ell}, G_{n}\right)=\frac{\mathrm{emb}^{+}\left(F_{\ell}, G_{n}\right)}{n_{\left(\left|F_{\ell}\right|\right)} p^{e\left(F_{\ell}\right)}} \sim \frac{\mathrm{emb}^{+}\left(F_{\ell}, G_{n}\right)}{n_{(|F|)} n^{\left|F_{\ell}\right|-|F|} p^{e\left(F_{\ell}\right)}}=\mathbb{E}\left(\prod_{u_{i} u_{j} \in E(F)} X_{i j}\right) .
$$

Equation (54) says, roughly speaking, that each $X_{i j}$ is close in probability to 'what it should be' (a random variable depending on $\kappa$ ). We should like to deduce that the expectation of the product is close to what it should be.

Let $Z$ be the set of $k$-tuples $\left(v_{1}, \ldots, v_{k}\right)$ with the $v_{i}$ distinct, such that $\left(v_{i}, v_{j}\right) \in S$ for some $1 \leq i<j \leq k$. Regarding $Z$ as an event in our probability space,

$$
\mathbb{P}(Z) \leq\left(\begin{array}{l}
k \\
2
\end{array}\right) \mathbb{P}\left(\left(v_{1}, v_{2}\right) \in S\right) \leq \varepsilon\left(\begin{array}{l}
k \\
2
\end{array}\right),
$$

from (54). Hölder's inequality thus gives

$$
\mathbb{E}\left(1_{Z} \prod_{u_{i} u_{j} \in E(F)} X_{i j}\right) \leq\left(\mathbb{E}\left(1_{Z}^{e(F)+1}\right) \prod_{u_{i} u_{j} \in E(F)} \mathbb{E}\left(X_{i j}^{e(F)+1}\right)\right)^{1 /(e(F)+1)},
$$

where $1_{Z}$ is the indicator function of the event $Z$. Now, for each $i$ and $j$, we have

$$
\mathbb{E}\left(X_{i j}^{e(F)+1}\right)=\frac{1}{n(n-1)} \sum_{u} \sum_{v \neq u}\left(\frac{p_{\ell}(u, v)}{n^{\ell-1} p^{\ell}}\right)^{e(F)+1},
$$

which is $O(1)$ by our assumption (53). Also, $\mathbb{E}\left(1_{Z}^{e(F)+1}\right)=\mathbb{E}\left(1_{Z}\right)=\mathbb{P}(Z) \leq \varepsilon$. Hence,

$$
\mathbb{E}\left(1_{Z} \prod_{u_{i} u_{j} \in E(F)} X_{i j}\right)=O\left(\varepsilon^{1 /(e(F)+1)}\right) .
$$

In other words, the contribution to (55) from semiembeddings mapping some edge of $F$ into a pair $(u, v) \in S$ is negligible. By definition of $S$, the contribution from all other semiembeddings is 'what it should be', and it follows that

$$
\left|s_{p}^{+}\left(F_{\ell}, G_{n}\right)-s\left(F_{\ell}, \kappa\right)\right| \leq O\left(\varepsilon^{1 /(e(F)+1)}\right)+O(\varepsilon) .
$$

Since $\varepsilon>0$ was arbitrary, we thus have $s_{p}^{+}\left(F_{\ell}, G_{n}\right) \sim s\left(F_{\ell}, \kappa\right)$. 
In the end, of course, it is $s_{p}\left(F_{\ell}, G_{n}\right)$ that we wish to bound, not $s_{p}^{+}\left(F_{\ell}, G_{n}\right)$. Since $s_{p}\left(F_{\ell}, G_{n}\right) \leq s_{p}^{+}\left(F_{\ell}, G_{n}\right)$ it remains to show that most semiembeddings are in fact embeddings, i.e., that the paths in $G_{n}$ making up a typical semiembedding are internally vertex disjoint. For paths corresponding to vertex disjoint edges of $F$, this is quite easy, using the fact that $s_{p}\left(T, G_{n}\right)$ is bounded for each tree, which tells us that almost all pairs of paths of length $\ell$ are vertex disjoint. For paths corresponding to edges of $F$ sharing a vertex, there is a similar argument. We shall not spell these arguments out as there is a third case that cannot be handled in this way, namely paths corresponding to duplicate edges in $F$. We must allow these, since we include, for example, $C_{2 \ell}$ in $F_{\ell}$. It is in handling these parts that our 'crude' partition $\left(Q_{i}\right)$ comes in.

Let us classify paths $w_{0} w_{1}, \ldots, w_{\ell}$ in $G_{n}$ into $N^{\ell+1}$ types, according to which part $Q_{i}$ each $w_{i}$ lies in. Let $S^{\prime}$ be the set of pairs $(u, v), u \neq v$, with the property that for all $N^{\ell-1}$ possible types of $u-v$ path, the number of $u-v$ paths of this type is 'close' to what it should be, i.e., within $\varepsilon\left|Q_{1}\right|^{\ell-1} p^{\ell} \sim \varepsilon n^{\ell-1} p^{\ell} / N^{\ell-1}$ of what it should be. As usual, 'what it should be' means the expected number in $G_{p}(n, \kappa)$, which depends not only on which parts $P_{i}$ the vertices $u$ and $v$ lie in, but also on the type of path being considered.

Since $N$ is fixed before $\eta$ is chosen, it is not hard to see that the argument giving (54) (applied with $\varepsilon / N^{\ell-1}$ in place of $\varepsilon$ ) also shows that $\left|S^{\prime}\right| \leq \varepsilon n(n-1)$; we omit the details. In other words, almost all pairs of vertices are joined by about the right number of paths of any given type. As before, we break down the set of embeddings of $F_{\ell}$ into $G_{n}$ according to which vertices $v_{1}, \ldots, v_{k}$ of $G_{n}$ the 'branch vertices' $u_{1}, \ldots, u_{k}$ are mapped to. Defining $Z^{\prime}$ analogously to $Z$, but using $S^{\prime}$ instead of $S$, the argument giving (56) shows that we may assume that $\left(v_{1}, \ldots, v_{k}\right) \notin Z^{\prime}$, i.e., that no pair $\left(v_{i}, v_{j}\right)$ is in $S^{\prime}$. Counting embeddings with $v_{1}, \ldots, v_{k}$ fixed, it remains to choose $e(F)$ paths joining the appropriate pairs $v_{i}, v_{j}$. Let us choose these paths one by one. Since the total number of paths joining $v_{i}$ to $v_{j}$ is about what it should be, all we must show is that few (say at most $\varepsilon n^{\ell-1} p^{\ell}$ ) paths from $v_{i}$ to $v_{j}$ meet one of our at most $e(F)-1$ earlier paths. But this is now easy: we must avoid a set $X$ of at most $(e(F)-1)(\ell-1)=O(1)$ vertices, the internal vertices of the previously chosen paths. In fact, we shall do much more, avoiding any part $Q_{a}$ that meets $X$ ! This rules out at most $(\ell-1)|X| N^{\ell-2}$ of the $N^{\ell-1}$ types of $v_{i}-v_{j}$ paths. Choosing $N$ large enough (larger than $1 / \varepsilon$ ), this is only a fraction $O(\varepsilon)$ of all possible types. Since $\left(v_{i}, v_{j}\right) \notin S^{\prime}$, we have almost the right number of paths of each remaining type, and hence almost the right number of paths in total. This completes our outline proof of Theorem 5.16.

Of course, there is a variant of Theorem 5.16 which is to Theorem 5.16 as Theorem 5.14 is to Theorem 5.13 we shall not state this separately.

Let us close this section by giving one simple example of a setting in which the conditions of Theorem 5.16 are satisfied. Let us suppose that our sequence $\left(G_{n}\right)$ has the following two properties. Firstly, the maximal degree $\Delta\left(G_{n}\right)$ is not too large:

$$
\Delta\left(G_{n}\right) \leq M p n,
$$


for some constant $M$. Secondly,

$$
p_{\ell-1}(u, v) \leq M n^{\ell-2} p^{\ell-1}
$$

for all $u \neq v \in V\left(G_{n}\right)$. Condition (57) is called DEG in Chung and Graham [19]; condition (58) is related to their condition $U(\ell)$. However, as discussed earlier, while superficially similar, (58) is much weaker than the condition $U(\ell)$. In particular, it is easy to check that if $p=n^{-\alpha}$ with $0<\alpha<1$ constant, and $\kappa$ is any bounded kernel, then the random graphs $G_{p}(n, \kappa)$ satisfy (57) and (58) with probability 1 , as long as $\alpha<1-1 /(\ell-1)$. If (57) and (58) hold then $p_{\ell}(v, w) \leq M^{2} n^{\ell-1} p^{\ell}$, for all $v$ and $w$, while $s_{p}\left(T, G_{n}\right) \leq M^{e(T)}$ for any tree $T$, so the conditions of Theorem 5.16] are satisfied. Similarly, $p_{t}(v, w) \leq M^{t-\ell+2} n^{t-1} p^{t}$ holds for all $t \geq \ell$, so the variant of Theorem 5.16 corresponding to Theorem 5.14 applies.

It follows that conditions (57) and (58) provide an answer to Question 5.8 Indeed, Theorem 5.16 tells us that, under these conditions, if $\kappa$ is a bounded kernel, then $d_{\text {cut }}\left(G_{n}, \kappa\right) \rightarrow 0$ implies $s_{p}\left(F, G_{n}\right) \rightarrow s\left(F, G_{n}\right)$ for all $F \in F_{\ell}$; its variant gives us $s_{p}\left(F, G_{n}\right) \rightarrow s\left(F, G_{n}\right)$ for all $F \in F_{\geq \ell}$. By Theorem 5.1 the counts $s(F, \kappa), F \in F_{\geq \ell}$, do determine the kernel (up to equivalence), so conditions (57) and (58) are 'suitable' in the sense of Question 5.8. As noted after Question 5.8, this implies the following result.

Theorem 5.17. Fix $\ell \geq 2$, let $p=p(n)$ be any function, and let $\left(G_{n}\right)$ be a sequence of graphs satisfying (57), (58) and the bounded density assumption, Assumption 4.1. Then, for any bounded kernel $\kappa$, we have $d_{\text {cut }}\left(G_{n}, \kappa\right) \rightarrow 0$ if and only if $d_{\mathrm{sub}}\left(G_{n}, \kappa\right) \rightarrow 0$, where $d_{\mathrm{sub}}$ is defined using $\mathcal{A}=\mathcal{T} \cup \mathcal{F}_{\geq \ell}$ for the set of admissible graphs.

In the next section we turn to what turns out to be a rather different topic, namely the study of metrics on graphs with $O(n)$ edges.

\section{The extremely sparse case}

When studying, for example, the random graph $G(n, p)$, there are many possibilities for $p$ as a function of $n$; which is most natural depends on what kind of properties one is interested in. Nevertheless, there are two canonical ranges of particular interest: the dense case, $p=\Theta(1)$, and the (extremely) sparse case, $p=\Theta(1 / n)$, the minimum sensible density. Here we are not studying random graphs, but it is still true that the most natural special cases are the densest graphs, those with $\Theta\left(n^{2}\right)$ edges, studied by Lovász and Szegedy [35] and Borgs, Chayes, Lovász, Sós and Vesztergombi [17, 18], for example, and the sparsest graphs, those with $\Theta(n)$ edges, as studied by Bollobás, Janson and Riordan [10. In previous sections, we studied sparse graphs with more than $\Theta(n)$ edges; in this section we turn to the sparsest graphs, taking $p=p(n)=1 / n$ as our normalizing density. 
One might expect that graphs with $\Theta(n)$ edges are somehow simpler than denser graphs, but in fact the reverse is often the case, particularly for the random graph $G(n, p)$. As a trivial example, note that there is significant variation in the vertex degrees in $G(n, c / n)$, while the degrees of $G(n, p)$ are concentrated around their mean if $n p \rightarrow \infty$. For this reason, we expect graphs with $\Theta(n)$ edges to be much harder to work with in the present context, which turns out to be the case. Indeed, as we shall see, very little of the material in earlier sections carries over.

One advantage of the extremely sparse case is that there is a unique natural normalization: in the notation of the previous sections, we fix $p=1 / n$ throughout this section (except for the digression in Subsection 6.4.1). Thus, for example, if $T$ is a tree, then

$$
t_{p}\left(T, G_{n}\right)=\operatorname{hom}\left(T, G_{n}\right) n^{e(T)-|T|}=\operatorname{hom}\left(T, G_{n}\right) / n .
$$

We shall discuss the role of small subgraphs containing cycles later.

Note that the random graph $G_{p}(n, \kappa)$ defined in Subsection 3.2 is now a special case of the sparse inhomogeneous model of Bollobás, Janson and Riordan [10. In this special case we choose $x_{1}, \ldots, x_{n}$ independently and uniformly from $[0,1]$, and then, conditional on this choice, join each pair $\{i, j\}$ of vertices independently, with probability $\min \left\{\kappa\left(x_{i}, x_{j}\right) / n, 1\right\}$, or simply $\kappa\left(x_{i}, x_{j}\right) / n$ if $n \geq \sup \kappa$. In [10] the sequence $x_{1}, \ldots, x_{n}$ is not assumed to be iid, so the model there is much more general. (On the other hand, in 10 there are certain technical assumptions, including that $\kappa$ is continuous almost everywhere. These assumptions are not needed here, since the iid sequence case is always well behaved; see the discussion in [11.)

We shall discuss several metrics in turn, starting with the cut metric.

\subsection{The cut metric and Szemerédi's Lemma}

In the dense and intermediate ranges, one of the key results used in the study of the cut metric is some form of Szemerédi's Lemma. In the extremely sparse setting, there is no way to apply Szemerédi's Lemma: the 'bounded density' assumption we imposed earlier, Assumption 4.1. can only be satisfied if $e\left(G_{n}\right)=$ $o(n)$, and there is no reasonable way to define an $(\varepsilon, p)$-regular partition so that such a thing exists at all! Correspondingly, many of the nice properties of the cut metric fail when $p=1 / n$, as we shall now see.

As before, given a graph $G$ with $n$ vertices, let $\kappa_{G}$ be the corresponding piecewise constant kernel, taking the values 0 and $1 / p=n$. Given a kernel $\kappa$, let $d_{\text {cut }}(G, \kappa)=d_{\text {cut }}\left(\kappa_{G}, \kappa\right)$. In the denser cases, under suitable assumptions, any sequence $\left(G_{n}\right)$ had a subsequence converging to some (bounded) kernel. Here such convergence is impossible, except in the trivial case where $\kappa=0$ almost everywhere (in which case $d_{\text {cut }}\left(G_{n}, \kappa\right) \rightarrow 0$ simply says that $e\left(G_{n}\right)=o(n)$ ). This is easy to see for bounded kernels (using Lemma 4.2), but in fact holds for arbitrary kernels. 
Theorem 6.1. Set $p=1 / n$, let $\kappa$ be a symmetric measurable function on $[0,1]^{2}$ with $0<\int \kappa<\infty$, and let $\left(G_{n}\right)$ be a sequence of graphs with $\left|G_{n}\right|=n$. Then $d_{\text {cut }}\left(G_{n}, \kappa\right)$ is bounded away from zero.

Proof. Suppose not; then, passing to a subsequence, we have $d_{\text {cut }}\left(G_{n}, \kappa\right) \rightarrow 0$. Hence there are rearrangements $\tau_{n}$ such that

$$
\left\|\kappa_{G_{n}}-\kappa^{\left(\tau_{n}\right)}\right\|_{\text {cut }} \rightarrow 0
$$

Note that $e\left(G_{n}\right) / n \rightarrow \int \kappa$, since

$$
d_{\text {cut }}\left(G_{n}, \kappa\right) \geq\left|\int \kappa_{G_{n}}-\int \kappa\right|=\left|2 e\left(G_{n}\right) / n-\int \kappa\right| .
$$

Hence $G_{n}$ has $\Theta(n)$ edges.

Let $M_{n}$ be a largest matching in $G_{n}$. We claim that there is a constant $c>0$ such that, for $n$ large enough, $M_{n}$ contains at least $c n$ edges. Otherwise, passing to a subsequence, we may assume that $\left|M_{n}\right| / n \rightarrow 0$. Writing $A_{n}$ for the vertex set of $M_{n}$, and $B_{n}$ for its complement, we have $e\left(B_{n}, B_{n}\right)=0$. Let $X_{n}$ be the subset of $[0,1]$ corresponding to $B_{n}$ under the rearrangement $\tau_{n}$. Then, from (59), $\int_{X_{n} \times X_{n}} \kappa \rightarrow 0$. Writing $\mu$ for Lebesgue measure, we have $\mu\left(X_{n}\right)=\left|B_{n}\right| / n \rightarrow 1$, so from basic properties of integration it follows that $\int_{X_{n} \times X_{n}} \kappa \rightarrow \int_{[0,1]^{2}} \kappa$, which is positive by assumption. This contradiction proves the claim.

Fix $c>0$ for which the claim above holds. Since $\kappa$ is integrable, we have $\int \kappa 1_{\{\kappa>C\}} \rightarrow 0$ as $C \rightarrow \infty$, where $1_{\{\kappa>C\}}:[0,1]^{2} \rightarrow\{0,1\}$ is the indicator of the event that $\kappa(x, y)>C$. In particular, there is a $C<\infty$ with $\int \kappa 1_{\{\kappa>C\}} \leq c / 4$. Fix an $n$ with $n>4 C / c$, noting that if $S \subset[0,1]^{2}$ satisfies $\mu(S) \leq 1 / n$, then

$$
\int_{S} \kappa \leq C \mu(S)+\int \kappa 1_{\{\kappa>C\}} \leq C / n+c / 4 \leq c / 2 .
$$

Choosing $n$ large enough, we may assume from (59) that there is a $\kappa \sim \kappa^{\prime}$ with

$$
\left\|\kappa_{G_{n}}-\kappa^{\prime}\right\|_{\text {cut }} \leq c / 25 \text {. }
$$

Let $\left\{u_{1} w_{1}, \ldots, u_{r} w_{r}\right\}$ be a matching in $G_{n}$ with $r \geq c n$; such a matching exists by our claim. Let $U=\left\{u_{i}\right\}$ and $W=\left\{w_{i}\right\}$. Identifying subsets of $V(G)$ with subsets of $[0,1]$ as usual, from (61) we have

$$
\left|\int_{U \times W} \kappa^{\prime}-e_{G_{n}}(U, W) / n\right| \leq c / 25 .
$$

Let $U^{\prime}$ be a random subset of $U$ obtained by selecting each vertex independently with probability $1 / 2$, and let $W^{\prime}$ be the complementary subset of $W$, defined by $W^{\prime}=\left\{w_{i}: u_{i} \notin U_{i}\right\}$. The edges of our matching never appear as edges from $U^{\prime}$ to $W^{\prime}$. On the other hand, any other edge $u_{i} w_{j}, i \neq j$, from $U$ to $W$ has probability $1 / 4$ of appearing. Hence,

$$
\mathbb{E}\left(e_{G_{n}}\left(U^{\prime}, W^{\prime}\right)\right)=e_{G_{n}}(U, W) / 4-r / 4 .
$$


Similarly, writing $S \subset[0,1]^{2}$ for the union of the $r 1 / n$ by $1 / n$ squares corresponding to the edges $u_{i} w_{i}$, we have

$$
\mathbb{E}\left(\int_{U^{\prime} \times W^{\prime}} \kappa^{\prime}\right)=\frac{1}{4} \int_{U \times W} \kappa^{\prime}-\frac{1}{4} \int_{S} \kappa^{\prime} .
$$

Combining the last three displayed equations using the triangle inequality, and noting that $\mu(S)=r / n^{2} \leq 1 / n$, it follows that

$$
\begin{aligned}
\left|\mathbb{E}\left(\int_{U^{\prime} \times W^{\prime}} \kappa^{\prime}\right)-\frac{1}{n} \mathbb{E}\left(e_{G_{n}}\left(U^{\prime}, W^{\prime}\right)\right)\right| & \geq \frac{r}{4 n}-\frac{1}{4} \int_{S} \kappa^{\prime}-c / 100 \\
& \geq c / 4-c / 8-c / 100>c / 16,
\end{aligned}
$$

using (60). On the other hand, from (61),

$$
\left|\int_{U^{\prime} \times W^{\prime}} \kappa^{\prime}-e_{G_{n}}\left(U^{\prime}, W^{\prime}\right) / n\right| \leq c / 25
$$

always holds, which implies a corresponding upper bound on the difference of the expectations. Since $c / 25<c / 16$, we obtain a contradiction, completing the proof.

The argument above in fact shows much more.

Theorem 6.2. With $p=1 / n$, a sequence $\left(G_{n}\right)$ of graphs with $\left|G_{n}\right|=n$ is Cauchy with respect to $d_{\text {cut }}$ if and only if $e\left(G_{n}\right)=o(n)$.

Proof. If $e\left(G_{n}\right)=o(n)$ then $\left(G_{n}\right)$ is trivially Cauchy, so we may assume that $\left(G_{n}\right)$ is Cauchy.

On the one hand, if there is some $c>0$ such that infinitely many $G_{n}$ contain a matching of size at least $c n$ then, passing to a subsequence, we may assume that all $G_{n}$ do. The argument above then shows that for any bounded kernel $\kappa$, if $n$ is large enough then $d_{\text {cut }}\left(\kappa_{G_{n}}, \kappa\right)>c / 25$. Applying this with $\kappa=\kappa_{G_{m}}$ shows that $\left(G_{n}\right)$ cannot be Cauchy.

On the other hand, if the largest matching in $G_{n}$ has size $o(n)$, then $G_{n}$ contains $n-o(n)$ vertices spanning no edges, from which it follows that lim inf $d_{\text {cut }}\left(\kappa, G_{n}\right) \geq$ $\int \kappa$ for any fixed kernel $\kappa$. Taking $\kappa=\kappa_{G_{m}}$, since $\left(\kappa_{G_{n}}\right)$ is Cauchy it follows that $\int \kappa_{G_{m}} \rightarrow 0$ as $m \rightarrow \infty$, i.e., that $e\left(G_{n}\right)=o(n)$.

Theorem 6.2 certainly shows that none of what we did with $d_{\text {cut }}$ in previous sections, and none of what Borgs, Chayes, Lovász, Sós and Vesztergombi [17, 18] did in the dense case, carries over to the extremely sparse case. It may still make sense, however, to use $d_{\text {cut }}$ as a measure of the similarity of two graphs $G, G^{\prime}$ with the same number $n$ of vertices. For this purpose, as before, $\widehat{d}_{\text {cut }}$ is more natural than $d_{\text {cut }}$; recall that $\widehat{d}_{\text {cut }}\left(G_{1}, G_{2}\right)$ is simply the minimum over graphs $G_{2}^{\prime}$ with $V\left(G_{2}^{\prime}\right)=V\left(G_{1}\right)$ and $G_{2}^{\prime}$ isomorphic to $G_{1}$ of the maximum over $S, T \subset V\left(G_{1}\right)$ of $\left|e_{G_{1}}(S, T)-e_{G_{2}}(S, T)\right| / n$. In this extremely sparse case, it is not so clear what it means for $\widehat{d}_{\text {cut }}\left(G_{n}, G_{n}^{\prime}\right)$ to tend to zero, if $G_{n}, G_{n}^{\prime}$ are graphs with $n$ vertices. For example, consider the following concrete question: 
Fix $c>0$, and let $G_{n}$ and $G_{n}^{\prime}$ be independent instances of the Erdős-Rényi random graph $G(n, c / n)$. Does the expected value of $\widehat{d}_{\text {cut }}\left(G_{n}, G_{n}^{\prime}\right)$ tend to 0 as $n \rightarrow \infty$ ?

If we consider graphs that are even a little denser, i.e., $G(n, p)$ with $n p \rightarrow \infty$, then the answer is trivially yes, defining $\widehat{d}_{\text {cut }}$ with respect to this density, of course. Indeed, there is no need to rearrange the vertices of $G_{n}^{\prime}$ in this case: it is immediate from Chernoff's inequality that whp every cut in $G(n, p)$ has size within $o\left(n^{2} p\right)$ of its expectation. As we shall see, in the extremely sparse case the situation is rather different.

Firstly, with $p=c / n$, rearrangement is certainly necessary: we must match all but $o(n)$ of the $\left(e^{-c}+o_{\mathrm{p}}(1)\right) n$ isolated vertices of $G_{n}$ with isolated vertices of $G_{n}^{\prime}$. In fact, matching up almost all the small components of $G_{n}$ with isomorphic small components of $G_{n}^{\prime}$, it is not hard to see that the question above reduces to a question about the giant component of $G(n, c / n)$. In particular, if $c \leq 1$, then the answer is yes, for the rather uninteresting reason that $G_{n}$ can be made isomorphic to $G_{n}^{\prime}$ by adding and deleting $o_{\mathrm{p}}(n)$ edges. As we shall see, this is the only possibility for a positive answer!

For two graphs $G_{1}, G_{2}$ with the same number of vertices, the (normalized) edit distance between $G_{1}$ and $G_{2}$ is the minimum number of edge changes (additions or deletions) needed to turn one of the graphs into a graph isomorphic to the other, divided by $p n^{2}$ :

$$
d_{\text {edit }}\left(G_{1}, G_{2}\right)=\frac{1}{n^{2} p} \min \left\{\left|E\left(G_{1}\right) \Delta E\left(G_{2}^{\prime}\right)\right|: G_{2}^{\prime} \cong G_{2}\right\} .
$$

Usually, one would leave the edit distance unnormalized; here we normalize for consistency with our notation for $d_{\text {cut }}$. It seems that Axenovich, Kézdy and Martin [3] were the first to define the edit distance explicitly, although implicitly the notion had been used much earlier, e.g., by Erdős [25] and Simonovits [39] in 1966, and in many subsequent papers. If $\left|G_{1}\right|=\left|G_{2}\right|=n$, then, trivially, $d_{\text {cut }}\left(G_{1}, G_{2}\right) \leq 2 d_{\text {edit }}\left(G_{1}, G_{2}\right)$. In general, $d_{\text {cut }}$ may be much smaller than $d_{\text {edit }}$; for example, two independent instances of the dense random graph $G(n, 1 / 2)$ are very close in $d_{\text {cut }}$, but far apart in $d_{\text {edit }}$. One can construct sparse examples by 'cheating': take two instances of $G(\sqrt{n}, 1 / 2)$ padded with $n-\sqrt{n}$ isolated vertices.

Although $d_{\text {cut }}$ and $d_{\text {edit }}$ are in general very different, in the extremely sparse case it turns out that they are closely related, at least for well behaved graphs.

Lemma 6.3. Let $\left(G_{n}\right)$ and $\left(G_{n}^{\prime}\right)$ be sequences of graphs with $O(n)$ edges, with $\left|G_{n}\right|=\left|G_{n}^{\prime}\right|=n$. Suppose that any o(n) vertices of $G_{n}$ meet o $(n)$ edges of $G_{n}$, and that the same holds for $G_{n}^{\prime}$. Then $\widehat{d}_{\text {cut }}\left(G_{n}, G_{n}^{\prime}\right) \rightarrow 0$ if and only if $d_{\text {edit }}\left(G_{n}, G_{n}^{\prime}\right) \rightarrow 0$.

Proof. As noted above, $\widehat{d}_{\text {cut }}\left(G_{n}, G_{n}^{\prime}\right) \leq 2 d_{\text {edit }}\left(G_{n}, G_{n}^{\prime}\right)$, so our task is to show that if $\widehat{d}_{\text {cut }}\left(G_{n}, G_{n}^{\prime}\right) \rightarrow 0$, then $d_{\text {edit }}\left(G_{n}, G_{n}^{\prime}\right) \rightarrow 0$. Relabelling the vertices of $G_{n}^{\prime}$, we may assume that $\left\|\kappa_{G_{n}}-\kappa_{G_{n}^{\prime}}\right\|_{\text {cut }} \rightarrow 0$. 
Let $C$ be a constant such that $e\left(G_{n}\right), e\left(G_{n}^{\prime}\right) \leq C n$. Suppose first that $G_{n} \backslash G_{n}^{\prime}$ (or $G_{n}^{\prime} \backslash G_{n}$ ) contains a matching $M$ of size at least $c n$, for some constant $c>0$. Set $a=c /(2 C)>0$, and select each edge of $M$ with probability $a$, independently of the others. Write $M^{\prime}$ for the set of edges obtained, and $V$ for the vertex set of $M^{\prime}$. Then $\mathbb{E}\left(e\left(G_{n}[V]\right)\right)$, the expected number of edges of $G_{n}$ spanned by $V$, is at least acn, considering only edges in $M$. On the other hand, if $e \notin M$ has both ends in the vertex set of $M$, then the probability that both endpoints of $e$ are included in $V$ is $a^{2}$, so $\mathbb{E}\left(e\left(G_{n}^{\prime}[V]\right)\right) \leq a^{2} e\left(G_{n}^{\prime}\right) \leq a^{2} C n$. Recalling that $a=c /(2 C)$, it follows that

$$
\mathbb{E}\left(e\left(G_{n}[V]\right)-e\left(G_{n}^{\prime}[V]\right)\right) \geq a c n-a^{2} C n=c^{2} n /(4 C)=\Theta(n) .
$$

Choosing a set for which the random difference is at least its expectation, we see that $\left\|\kappa_{G_{n}}-\kappa_{G_{n}^{\prime}}\right\|_{\text {cut }}=\Theta(1)$, a contradiction.

From the above we may assume that the largest matchings in $G_{n} \backslash G_{n}^{\prime}$ and in $G_{n}^{\prime} \backslash G_{n}$ have size $o(n)$. But then there is a set $V$ of $o(n)$ vertices that meets every edge of the symmetric difference $G_{n} \Delta G_{n}^{\prime}$. By assumption, $V$ meets only $o(n)$ edges of $G_{n} \cup G_{n}^{\prime}$, so it follows that $E\left(G_{n} \Delta G_{n}^{\prime}\right)=o(n)$, giving $d_{\text {edit }}\left(G_{n}, G_{n}^{\prime}\right) \rightarrow$ 0 .

Note that Lemma 6.3 becomes false if the condition that $o(n)$ vertices meet $o(n)$ edges is omitted, as shown by the example mentioned earlier, where $G_{n}$ and $G_{n}^{\prime}$ are two instances of the random graph $G(\sqrt{n}, 1 / 2)$, each with $n-\sqrt{n}$ isolated vertices added.

Lemma 6.3 applies to two independent instances $G_{1}, G_{2}$ of the random graph $G(n, c / n)$. Hence, $\widehat{d}_{\text {cut }}\left(G_{1}, G_{2}\right) \stackrel{\mathrm{p}}{\rightarrow} 0$ if and only if $d_{\text {edit }}\left(G_{1}, G_{2}\right) \stackrel{\mathrm{p}}{\rightarrow} 0$. It is not too hard to see that the latter condition cannot hold for any $c>1$.

Theorem 6.4. For every $c>1$ there is a $\delta=\delta(c)>0$ such that, if $G_{1}$ and $G_{2}$ are independent instances of $G(n, c / n)$, then whp the unnormalized edit distance between $G_{1}$ and $G_{2}$ is at least $\delta n$.

In other words, normalizing with $p=1 / n$, we have $d_{\text {edit }}\left(G_{1}, G_{2}\right) \geq \delta$ whp.

Proof. Let us start with an observation about $G(n, c / n)$. Let $0<a<b$ be constants; we shall estimate the probability of the event $E_{\delta}(H)$ that $G_{2}=$ $G(n, c / n)$ contains all but at most $\delta n$ edges of some graph $H^{\prime}$ isomorphic to $H$, where $H$ is any given graph with $\lfloor a n\rfloor$ vertices and at least $b n$ edges. There are $\left(\begin{array}{c}n \\ \lfloor a n\rfloor\end{array}\right)$ choices for the vertex set of $H^{\prime}$, and then at most $\lfloor$ an $\rfloor$ ! graphs $H^{\prime}$ with this vertex set isomorphic to $H$. Finally, given $H^{\prime}$, there are crudely at most $\delta n\left(\begin{array}{c}e(H) \\ \delta n\end{array}\right)$ choices for the edges of $H^{\prime}$ to omit, while the probability that $G(n, c / n)$ contains the remaining edges is at most $(c / n)^{e(H)-\delta n}$. Hence,

$$
\begin{aligned}
\mathbb{P}\left(E_{\delta}(H)\right) & \leq\left(\begin{array}{c}
n \\
\lfloor a n\rfloor
\end{array}\right)\lfloor a n\rfloor !\left(\begin{array}{c}
e(H) \\
\delta n
\end{array}\right)(c / n)^{e(H)-\delta n} \\
& \leq n^{a n}(e b / \delta)^{\delta n}(c / n)^{(b-\delta n)}=n^{(a-b+\delta) n} e^{O(n)} .
\end{aligned}
$$

If $a, b$ and $\delta$ are constants with $\delta<b-a$, then the final probability is $o(1)$. 
Turning to the proof of the theorem, if $d_{\text {edit }}\left(G_{1}, G_{2}\right) \leq \delta$, then the event $E_{\delta}(H)$ holds for any $H \subset G_{1}$. If $c>2$, then $G_{1}$ itself has $n$ vertices and $c n / 2+o_{\mathrm{p}}(n)$ edges, so setting $a=1$ and $b=c^{\prime} n / 2$ for any $2<c^{\prime}<c$, whp $G_{1}$ has a subgraph $H$ with $e(H) \geq b n$. Setting $\delta=\left(c^{\prime}-2\right) / 2>0$, whp we have

$$
\mathbb{P}\left(d_{\text {edit }}\left(G_{1}, G_{2}\right) \leq \delta \mid G_{1}\right) \leq \mathbb{P}\left(E_{\delta}(H)\right)=o(1),
$$

from which the result follows.

If $1<c \leq 2$, standard results imply that there are functions $\rho(c)$ and $\zeta(c)$ with $0<\rho(c)<\zeta(c)$ such that the largest component of $G(n, c / n)$ has $\rho(c) n+o_{\mathrm{p}}(n)$ vertices and $\zeta(c) n+o_{\mathrm{p}}(n)$ edges. Fixing $a<b$ with $\rho(c)<a<$ $b<\zeta(c)$, it follows that whp $G_{1}=G(n, c / n)$ contains a subgraph $H$ with at most $a n$ vertices and at least $b n$ edges. Taking $\delta=(b-a) / 2>0$, the result follows as above.

Using the results of Bollobás, Janson and Riordan [10, the proof above may be extended easily to the much more general model $G_{1 / n}(n, \kappa)$, although one first needs to decide what the appropriate statement is. As in [10], let $T_{\kappa}$ be the integral operator associated to $\kappa$, defined by (33), and let $\left\|T_{\kappa}\right\|_{2}$ be its $L_{2}$-norm. Roughly speaking, it was shown in [10] that $G_{1 / n}(n, \kappa)$ has a giant component if and only if $\left\|T_{\kappa}\right\|>1$. (There is a slight caveat here: the results of [10] assume that $\kappa$ is continuous almost everywhere; this assumption is only needed due to the more general choice of the vertex types made there. It is easy to see that all these results apply to general bounded $\kappa$ if we choose the vertex types iid, as we do in the definition of $G_{1 / n}(n, \kappa)$; this is discussed in [1].)

More precisely, it is shown in [10] that if $\kappa$ satisfies a certain irreducibility condition, then $G_{1 / n}(n, \kappa)$ has a 'giant component' with $\rho(\kappa) n+o_{\mathrm{p}}(n)$ vertices and $\zeta(\kappa) n+o_{\mathrm{p}}(n)$ edges, for constants $\rho(\kappa)$ and $\zeta(\kappa)$ satisfying $0<\rho(\kappa)<\zeta(\kappa)$ whenever $\left\|T_{\kappa}\right\|>1$ (see Theorems 3.1 and 3.5 and Proposition 10.1 in [10]). Since any kernel effectively contains an irreducible kernel (see Lemma 5.17 of [10]), it follows that if $\kappa$ is any bounded kernel with $\left\|T_{\kappa}\right\|>1$, then there are constants $0<a<b$ depending only on $\kappa$ such that, whp, $G_{1}=G_{1 / n}(n, \kappa)$ has a (connected) subgraph $H$ with at most an vertices and at least $b n$ edges. The observation at the start of the proof of Theorem 6.4 shows that, for any $c$, the probability that $G(n, c / n)$ contains almost all of such a graph $H$ is $o(1)$. Since we may couple $G_{2}=G_{1 / n}(n, \kappa)$ and $G(n, \sup \kappa / n)$ so that the former is a subgraph of the latter, it follows that whp independent copies $G_{1}$ and $G_{2}$ of $G_{1 / n}(n, \kappa)$ are well separated in $d_{\text {edit }}$. Using Lemma 6.3, it follows that they are well separated in $d_{\text {cut }}$. In other words, we have proved the following result.

Theorem 6.5. Let $\kappa$ be a bounded kernel with $\left\|T_{\kappa}\right\|>1$, and let $G_{n}$ and $G_{n}^{\prime}$ be independent instances of $G_{1 / n}(n, \kappa)$. Then there is a constant $\delta>0$ such $d_{\text {edit }}\left(G_{n}, G_{n}^{\prime}\right) \geq \delta$ and $d_{\text {cut }}\left(G_{n}, G_{n}^{\prime}\right) \geq \delta$ hold whp.

In fact, standard martingale arguments show that each of $d_{\text {edit }}\left(G_{n}, G_{n}^{\prime}\right)$ and $d_{\text {cut }}\left(G_{n}, G_{n}^{\prime}\right)$ is concentrated about its mean, which is thus bounded away from zero. We omit the details, which are very similar to those in the proof of Theorem 6.15 below. 
As noted above for $G(n, c / n)$, the condition $\left\|T_{\kappa}\right\|>1$ is necessary: otherwise, from the results of Bollobás, Janson and Riordan [10, $G_{1 / n}(n, \kappa)$ consists almost entirely of small tree components, with the number of copies of any given tree concentrated. It follows easily that $d_{\text {edit }}\left(G_{n}, G_{n}^{\prime}\right)$ and hence $d_{\text {cut }}\left(G_{n}, G_{n}^{\prime}\right)$ converge to 0 in probability (and almost surely) in this case.

\subsection{Tree counts}

Since it seems we cannot do much with $d_{\text {cut }}$ in the extremely sparse case, let us now turn our attention to $d_{\text {sub }}$. Let $F$ be a connected graph which is not a tree. The denominator in the definition of $t_{p}\left(F, G_{n}\right)$ or $s_{p}\left(F, G_{n}\right)$ is $\Theta\left(n^{|F|} p^{e(F)}\right)$, which is order 1 if $F$ is unicyclic, and tends to zero if $F$ contains two or more cycles. This suggests that, in this range, the parameters $s_{p}(F, \cdot)$ and $t_{p}(F, \cdot)$ make sense only if $F$ is a tree. For example, with $F$ unicyclic, convergence of $s_{p}\left(F, G_{n}\right)$ simply means that for large $n$, every $G_{n}$ contains the same number of copies of $F$. This condition is very far from the kind of global graph property we are looking for. Since the expected number of copies of a connected graph $F$ in $G(n, c / n)$ tends to infinity if and only if $F$ is tree, roughly speaking we do not expect to see small cycles in graphs with $\Theta(n)$ edges. Of course, there are natural examples of extremely sparse graphs containing many short cycles, but we should handle these differently; see Subsection 6.6. For now, we shall consider graphs that, like $G(n, c / n)$, contain few short cycles. More formally, throughout this subsection we assume that $\left(G_{n}\right)$ is asymptotically treelike, in the sense that

$$
\operatorname{emb}\left(F, G_{n}\right)=o(n)
$$

for any connected $F \in \mathcal{F}$ that is not a tree. Under a suitable assumption on the degrees in $G_{n}$, it suffices to impose condition (63) for cycles.

Under the assumption (63), it is easy to see that the parameters $\left(s_{p}\left(T, G_{n}\right)\right)_{T \in \mathcal{T}}$ and $\left(t_{p}\left(T, G_{n}\right)\right)_{T \in \mathcal{T}}$ are essentially equivalent. In particular, up to a $o(1)$ error, for any tree $T, t_{p}\left(T, G_{n}\right)$ can be written as a linear combination of the parameters $s_{p}\left(T^{\prime}, G_{n}\right),\left|T^{\prime}\right| \leq|T|$, and vice versa. We shall work with $s_{p}\left(T, G_{n}\right)$, which is more natural. Adjusting the normalizing constant very slightly, we shall simply set

$$
s_{p}\left(T, G_{n}\right)=\operatorname{emb}\left(T, G_{n}\right) / n .
$$

As before, we shall assume that $\sup _{n} s_{p}\left(T, G_{n}\right) \leq c_{T}<\infty$ for each tree $T$. In fact, it will be convenient to make the stronger assumption that the tree counts are exponentially bounded, i.e., that there is a constant $C$ such that $\limsup s_{p}\left(T, G_{n}\right) \leq C^{e(T)}$ for every tree $T$. For example, taking $T$ to be a star, this condition implies that the $k$ th moment of the degree of a random vertex of $G_{n}$ is at most $C^{k}+o(1)$ as $n \rightarrow \infty$. As before (see (15)), enumerating the set $\mathcal{T}$ of isomorphism classes of finite trees as $T_{1}, T_{2}, \ldots$, we may define a map

$$
s_{p}: \mathcal{F} \rightarrow X, \quad G \mapsto\left(s_{p}\left(T_{i}, G\right)\right)_{i=1}^{\infty},
$$

where $X=\prod_{i}\left[0, c_{T_{i}}\right]$, and we may define the (tree) subgraph distance $d_{\text {sub }}\left(G_{1}, G_{2}\right)$ between two graphs $G_{1}$ and $G_{2}$ as $d\left(s_{p}\left(G_{1}\right), s_{p}\left(G_{2}\right)\right)$, where $d$ is any metric on 
$X$ giving rise to the product topology. (It is not clear whether $d_{\text {sub }}$ is a metric or only a pseudometric, but this is not important.) Again, as before, we may extend $s_{p}$ to bounded kernels $\kappa$ by mapping $\kappa$ to $\left(s\left(T_{i}, \kappa\right)\right)_{i=1}^{\infty}$, and consider the question of when $d_{\mathrm{sub}}\left(G_{n}, \kappa\right) \rightarrow 0$.

Unlike the cut metric, it is certainly possible to have convergence in the metric $d_{\text {sub }}$. Indeed, it is easy to check that $s_{p}\left(T, G_{1 / n}(n, \kappa)\right)$ converges in probability to $s(T, \kappa)$ for any tree $T$ and any bounded kernel $\kappa$, and one can then show that $d_{\text {sub }}\left(G_{1 / n}(n, \kappa), \kappa\right) \rightarrow 0$ with probability 1 .

In this subsection, the main questions we shall consider are: which points $X$ of $[0,1]^{\mathcal{T}}$ are realizable as limits of sequences $\left(s_{p}\left(G_{n}\right)\right)$, where $\left(G_{n}\right)$ is asymptotically treelike and has bounded tree counts, and how do these limit points relate to kernels? In fact, we shall reformulate these questions slightly.

Recall that in the definition of $\operatorname{emb}\left(T, G_{n}\right)$, the tree $T$ is labelled. Let us also regard $T$ as rooted, with root vertex 1 , say. Letting $v$ denote a vertex of $G_{n}$ chosen uniformly at random, $s_{p}\left(T, G_{n}\right)$ is simply the expected number of embeddings from $T$ into $G_{n}$ mapping the root to $v$. Letting $T$ run over stars, the numbers $s_{p}\left(T, G_{n}\right)$ give the moments of the degree of $v$; assuming these moments converge, and that the limiting moments are exponentially bounded, a standard result implies that they determine the limiting degree distribution. (For an example of two non-negative integer valued distributions with the same finite moments, see Janson [30], taking, for example, $\alpha=\log (2 / 3)$ and $\alpha=\log (4 / 5)$ in Example 2.12.) More generally, the parameters $s_{p}\left(T, G_{n}\right)$ for all finite trees $T$ provide a sort of moment characterization of the local neighbourhood of $v$. Presumably, if these tree counts are exponentially bounded, then they characterize the distribution of the local neighbourhood of $v$. (It should not be hard to adapt the proof that convergence of exponentially bounded moments implies convergence in distribution based on the Jordan-Bonferroni inequalities: consider the event that certain edges are present, forming a copy of some given tree $T$ with $v$ as the root, and apply inclusion-exclusion to calculate the probability that certain other edges are absent, so $T$ is the local neighbourhood of $v$; we have not yet checked the details.) In fact, for this something weaker than exponentially bounded tree counts should be enough, but exponential boundedness is a natural assumption, as it is the global analogue of the (perhaps too restrictive) local condition that all degrees are uniformly bounded. In any case, it makes more sense to study the distribution of local neighbourhoods directly, rather than its moments.

For $t \geq 0$, let $\Gamma_{\leq t}(v)$ denote the subgraph of $G_{n}$ formed by all vertices within graph distance $t$ of $v$, regarded as a rooted graph with root $v$. Let $\mathcal{T}_{t}^{\mathrm{r}}$ denote the set of isomorphism classes of rooted trees of height at most $t$, i.e., such that every vertex is within distance $t$ of the root. Finally, for $T \in \mathcal{T}_{t}^{\mathrm{r}}$, let

$$
p\left(T, G_{n}\right)=p_{t}\left(T, G_{n}\right)=\mathbb{P}\left(\Gamma_{\leq t}(v) \cong T\right),
$$

where $v$ is a vertex of $G_{n}$ chosen uniformly at random, and $\cong$ denotes isomorphism as rooted graphs. Officially, the subscript $t$ is necessary in the notation, since if $T \in \mathcal{T}_{t}^{\mathrm{r}} \subset \mathcal{T}_{t+1}^{\mathrm{r}}$, then $\Gamma_{\leq t}(v) \cong T$ and $\Gamma_{\leq t+1}(v) \cong T$ are different 
conditions. In practice, we work with the disjoint union $\mathcal{T}^{\mathrm{r}}$ of the sets $\mathcal{T}_{t}^{\mathrm{r}}$, $t=0,1,2, \ldots$, so each $T \in \mathcal{T}^{\mathrm{r}}$ comes with a height $t$ which we do not indicate in the notation; $T$ may or may not contain vertices at distance $t$ from the root.

Since each $p\left(T, G_{n}\right)$ lies in $[0,1]$, any sequence $\left(G_{n}\right)$ has a subsequence on which $p\left(T, G_{n}\right)$ converges for every $T$. We would like to study the set $\mathcal{P}$ of possible limits $(p(T))_{T \in \mathcal{T}^{\mathrm{r}}}$ arising from asymptotically treelike sequences with exponentially bounded tree counts.

We start with some simple observations. First, note that if $\left(G_{n}\right)$ is asymptotically treelike, then

$$
\sum_{T \in \mathcal{T}_{t}^{\mathrm{r}}} p\left(T, G_{n}\right) \rightarrow 1
$$

as $n \rightarrow \infty$ with $t$ fixed; this is simply the statement that only $o(n)$ vertices have cycles in their $t$-neighbourhoods. In general, such a statement does not carry over to the limit, since infinite sums are not continuous with respect to pointwise convergence. Recall that we are assuming that the tree counts in $\left(G_{n}\right)$ are (exponentially) bounded. It is easy to check that, under this assumption, for any $t \geq 0$ and any $\varepsilon>0$, there exists an $M$ such that, for large enough $n$, at most $\varepsilon n$ vertices $v$ have more than $M$ edges in their $t$-neighbourhoods. (In the terminology of probability theory, for each $t$ the sequence $\left(X_{n, t}\right)$ of random variables given by $X_{n, t}=e\left(\Gamma_{\leq t}(v)\right)$ is 'tight', where $v$ is a uniformly chosen random vertex of $G_{n}$. Equivalently, the random variables $\Gamma_{\leq t}(v), n=1,2, \ldots$, are themselves tight.) In other words, for large $n$, at least $1-\varepsilon$ of the mass of the distribution $\left(p\left(T, G_{n}\right)\right)_{T \in \mathcal{T}_{t}^{\mathrm{r}}}$ is concentrated on a fixed, finite set of trees. Under this condition, pointwise convergence implies convergence in $L_{1}$, and it follows that $\sum_{T \in \mathcal{T}_{t}^{\mathrm{r}}} p(T)=1$ whenever $(p(T)) \in \mathcal{P}$. In other words, $p$ gives a probability distribution on $\mathcal{T}_{t}^{\mathrm{r}}$ for each $t$.

Given a finite or infinite rooted tree $T$, we may define the restriction of $T$ to height $t$ to be the subtree $\left.T\right|_{t}$ of $T$ induced by all vertices within distance $t$ of the root, which we naturally regard as an element of $\mathcal{T}_{t}^{\mathrm{r}}$. If $\left(G_{n}\right)$ is asymptotically treelike, then, for any $T \in \mathcal{T}_{t}^{\mathrm{r}}$, we have

$$
p\left(T, G_{n}\right)=\sum_{T^{\prime} \in \mathcal{T}_{t+1}^{\mathrm{r}}:\left.T^{\prime}\right|_{t}=T} p\left(T^{\prime}, G_{n}\right)+o(1) .
$$

(The $o(1)$ correction appears because of the possibility that the $t+1$ neighbourhood of a random vertex $v$ contains a cycle while the $t$ neighbourhood does not.) Using $L_{1}$ convergence, it follows that

$$
p(T)=\sum_{T^{\prime} \in \mathcal{T}_{t+1}^{\mathrm{r}}:\left.T^{\prime}\right|_{t}=T} p\left(T^{\prime}\right)
$$

whenever $(p(T)) \in \mathcal{P}$. In other words, the distribution on $\mathcal{T}_{t}^{\mathrm{r}}$ is obtained from that on $\mathcal{T}_{t+1}^{\mathrm{r}}$ by the natural restriction operation.

This last fact allows us to combine the distributions on $\mathcal{T}_{t}^{\mathrm{r}}$ given by $p \in \mathcal{P}$ into a single probability distribution on the set $\mathcal{T}_{\infty}^{\mathrm{r}}$ of (finite or infinite) locally 
finite rooted trees. Of course, we take for the set of measurable events the $\sigma$-field generated by the sets

$$
E_{t, T}=\left\{T^{\prime} \in \mathcal{T}_{\infty}^{\mathrm{r}}:\left.T^{\prime}\right|_{t}=T\right\}
$$

for $t=0,1,2, \ldots$ and $T \in \mathcal{T}_{t}^{\mathrm{r}}$. This allows us ask the question: which probability distributions $\pi$ on $\mathcal{T}_{\infty}^{\mathrm{r}}$ arise as limits of sequences asymptotically treelike, exponentially bounded sequences $\left(G_{n}\right)$ ? In other words, when is there a sequence $\left(G_{n}\right)$ with the appropriate properties such that

$$
\lim _{n \rightarrow \infty} p_{t}\left(T, G_{n}\right)=\pi\left(E_{t, T}\right)
$$

holds for every $t \geq 0$ and every $T \in \mathcal{T}_{t}^{\mathrm{r}}$. (For a closely related question of Aldous and Lyons [1] see Subsection 6.6]) We shall give some examples of such distributions in the next section, arising from random graphs. Here, we give a trivial example: fix $d \geq 2$ and let $\pi$ be the distribution that is concentrated on one point of $\mathcal{T}_{t}^{\mathrm{r}}$, the (infinite) $d$-regular tree. This distribution arises as a limit in the sense above if we take $G_{n}$ to be a random $d$-regular graph with $n$ vertices.

Having given a trivial example of a distribution that can arise, let us give a trivial example of one that cannot. Let $\pi$ be any distribution concentrated on the two trees $T \in \mathcal{T}_{\infty}^{\mathrm{r}}$ in which every vertex has degree 2 or 3 , and the neighbours of a vertex of degree 2 have degree 3 and vice versa. (There are two such trees since our trees are rooted.) Considering graphs $G_{n}$ in which the vertices have degrees 2 and 3, there is no 'local reason' why the neighbourhoods of a random vertex $v$ shouldn't look like the first few generations of $\pi$. However, unless $\pi$ satisfies an extra condition, there is of course a global reason: there are as many edges from vertices of degree 2 to vertices of degree 3 as vice versa, so if all edges join vertices of different degrees, we must have $3 / 2$ times as many degree 2 vertices as degree 3 vertices. Thus, if $\pi$ is to arise as a limit, it must assign probabilities $3 / 5$ and $2 / 5$ to the trees in which the root has degree 2 and 3 , respectively.

More generally, consider the following two ways of picking a (not uniformly) random vertex of $G_{n}$. (A) pick a vertex $v$ with probability proportional to its degree. (B) pick a vertex $w$ with probability proportional to its degree, then choose an edge incident with $w$ uniformly, and let $v$ be the other end of this edge. It is easy to see that (A) and (B) give the same distribution for the vertex $v$ - indeed, we are simply choosing an edge $e$ of $G$ at random, and then picking an end of $e$ at random. In (B) we 'change our minds' after picking the random end, which makes no difference. The equivalence of $(\mathrm{A})$ and $(\mathrm{B})$ gives rise to a consistency condition on our distributions $\pi$. (For a discussion of various closely related conditions in the general graph setting, see Subsection 6.6.)

Let $\pi$ be any probability distribution on $\mathcal{T}_{\infty}^{\mathrm{r}}$ in which the expected degree of the root is finite. (Any distribution arising from some $p \in \mathcal{P}$ will have this property, due to the bounded average degree of graphs in $G_{n}$.) Then we may define the 'root-sized-biased' version $\widetilde{\pi}$ of $\pi$ to be the distribution $\pi$ biased by 
the degree of the root. In other words, for any $t \geq 1$ and $T \in \mathcal{T}_{t}^{\mathrm{r}}$,

$$
\widetilde{\pi}\left(E_{t, T}\right)=\frac{d_{0}(T) \pi\left(E_{t, T}\right)}{\mathbb{E}_{\pi}\left(d_{0}\right)},
$$

where the $d_{0}(T)$ denotes the degree of the root of $T$, and $\mathbb{E}_{\pi}\left(d_{0}\right)$ is simply the expectation of the degree of the root of a $\pi$-random $T \in \mathcal{T}_{\infty}^{\mathrm{r}}$. Let us define the shift $\tilde{\pi}^{*}$ of $\tilde{\pi}$ to be the distribution on $\mathcal{T}_{\infty}^{\mathrm{r}}$ obtained as follows: first choose a $T \in \mathcal{T}_{\infty}^{\mathrm{r}}$ according to the distribution $\tilde{\pi}$. Then pick a neighbour $v$ of the root uniformly from among the $d_{0}(T)$ neighbours. Finally, let $T^{\prime} \in \mathcal{T}_{\infty}^{\mathrm{r}}$ be the tree obtained from $T$ by taking $v$ as the root. Since the restriction of $T^{\prime}$ to height $t$ is determined by the restriction of $T$ to height $t+1$, it is easy to check that this does define a probability distribution $\widetilde{\pi}^{*}$ on $\mathcal{T}_{\infty}^{\mathrm{r}}$.

Using the equivalence of the procedures (A) and (B) above for picking a random vertex of $G_{n}$, it is easy to see that if $\pi$ arises as the local limit of one of our sequences $\left(G_{n}\right)$, then $\pi$ is shift invariant, in that $\widetilde{\pi}^{*}=\widetilde{\pi}$. We do not know whether this trivial necessary condition on $\pi$ is sufficient. (See also Question 6.21.)

Question 6.6. Does every shift-invariant probability distribution on $\mathcal{T}_{\infty}^{\mathrm{r}}$ arise as the local limit of some sequence $\left(G_{n}\right)$ of graphs with $\left|G_{n}\right|=n$ ?

The sequence $\left(G_{n}\right)$ above will necessarily be asymptotically treelike (otherwise the total weight of $\pi$ would be less than 1 , so $\pi$ would not be a probability distribution). However, in the question above we have lost the condition that the tree counts of $\left(G_{n}\right)$ be exponentially bounded. Such a condition may or may not be needed to get sensible limiting behaviour. To avoid possible complications, it thus makes sense to consider the following variant of Question 6.6.

Question 6.7. Let $\Delta \geq 2$ be given, and let $\pi$ be a shift-invariant probability distribution on the set of trees $T \in \mathcal{T}_{\infty}^{\mathrm{r}}$ with maximum degree at most $\Delta$. Must $\pi$ arise as the local limit of some sequence $\left(G_{n}\right)$ of graphs with all degrees at most $\Delta$ ?

Let us finish this subsection by noting that there is a certain class of distributions for which the answer to the questions above is trivially yes, namely the class of probability distributions $\pi$ on finite rooted trees. It is easy to check that a distribution $\pi$ on the set of finite rooted trees (a subset of $\mathcal{T}_{\infty}^{\mathrm{r}}$ ) corresponds in the natural way to a distribution on unrooted trees if and only if $\pi$ is shift invariant. In this case it is easy to construct forests $G_{n}$ with the appropriate limiting distribution of trees.

\subsection{Tree counts in random graphs}

We have seen in the previous subsection that the metric $d_{\text {sub }}$, defined using only tree counts, makes sense in the extremely sparse case $(p=1 / n)$, in that there are non-trivial convergent sequences: unlike the cut metric, $d_{\text {sub }}$ is not 'too strong' to be meaningful. Here we ask whether it is 'too weak' to be useful, i.e., 
whether too many sequences converge that 'should not'. Of course this is rather a vague question, so we concentrate on a precise special case: we have seen that for any bounded kernel we have $d_{\text {sub }}\left(G_{1 / n}(n, \kappa), \kappa\right) \rightarrow 0$ with probability 1 , so we ask: which kernels are distinguished by $d_{\text {sub }}$ ? In other words, when do two kernels $\kappa_{1}$ and $\kappa_{2}$ satisfy $s\left(T, \kappa_{1}\right)=s\left(T, \kappa_{2}\right)$ for every tree $T$ ? As in the previous section, we shall change viewpoint slightly, considering the distribution of local neighbourhoods, rather than counting subgraphs.

Adopting the terminology of Bollobás, Janson and Riordan [10], let $(\mathcal{S}, \mu)$ be an arbitrary probability space. By a kernel on $(\mathcal{S}, \mu)$ we mean a symmetric measurable function on $\mathcal{S} \times \mathcal{S}$. So far we have almost always taken $\mathcal{S}=[0,1]$ and $\mu$ Lebesgue measure, but the notation is more convenient if we are rather more general here. As in 10] (taking the special case where the vertex types are iid), suppressing the dependence on $(\mathcal{S}, \mu)$ in the notation, we may form a random graph $G_{1 / n}(n, \kappa)$ as follows: let $x_{1}, \ldots, x_{n} \in \mathcal{S}$ be iid with the distribution $\mu$, and then, given $\left(x_{1}, \ldots, x_{n}\right)$, join each pair of vertices $\{i, j\} \subset[n]^{(2)}$ with probability $\kappa(i, j) / n$, independently of the other pairs. We say that vertex $i$ has type $i$ and call $(\mathcal{S}, \mu)$ the type space.

Let $\mathfrak{X}_{\kappa}$ be the multi-type Galton-Watson branching process naturally associated to $\kappa$ : we start in generation 0 with a single particle whose type is distributed according to $\mu$. A particle of type $x$ has children whose types form a Poisson process on $\mathcal{S}$ with the distribution $\kappa(x, y) d \mu(y)$ : the number of such children in a measurable set $A \subset \mathcal{S}$ is Poisson with mean $\int_{A} \kappa(x, y) d \mu(y)$. As usual, the children of different particles are independent, and independent of the history. This branching process is the key to the analysis of the random graph $G_{1 / n}(n, \kappa)$ in [10].

The branching process $\mathfrak{X}_{\kappa}$ is of course simply a random rooted tree with labels attached to the vertices, giving the type of each vertex. Forgetting the labels, we may regard $\mathfrak{X}_{\kappa}$ as a random rooted tree; we write $\pi_{\kappa}$ for the corresponding probability measure on $\mathcal{T}_{\infty}^{\mathrm{r}}$. It is not hard to check that if $\kappa$ is bounded, then $\pi_{\kappa}$ provides the correct local approximation for $G_{1 / n}(n, \kappa)$ : in the notation of the previous subsection, for each $T \in \mathcal{T}_{t}^{\mathrm{r}}$ we have

$$
p_{t}\left(T, G_{1 / n}(n, \kappa)\right) \rightarrow \pi_{\kappa}\left(E_{t, T}\right) \text { with probability } 1 .
$$

This is the distributional equivalent of the convergence in moments given by $s_{p}\left(T, G_{1 / n}(n, \kappa)\right) \rightarrow s(T, \kappa)$ for every tree $T$.

In the light of the comments above, we should like to answer the following question: when do two different branching processes $\mathfrak{X}_{\kappa_{1}}$ and $\mathfrak{X}_{\kappa_{2}}$ give rise to the same random tree? In other words, when is $\pi_{\kappa_{1}}=\pi_{\kappa_{2}}$ ? It is not hard to check that, at least for bounded $\kappa$, the counts $(s(T, \kappa))_{T \in \mathcal{T}}$ determine $\pi_{\kappa}$ and vice versa, so this is the same question as that asked at the start of the section. Since $\pi_{\kappa}$ directly describes the local structure of $G_{1 / n}(n, \kappa)$, we consider this formulation more informative.

There is an obvious case when $\pi_{\kappa_{1}}=\pi_{\kappa_{2}}$ : let $\kappa_{i}$ be a kernel on $\left(\mathcal{S}_{i}, \mu_{i}\right)$. We say that $\kappa_{1}$ refines $\kappa_{2}$, and write $\kappa_{1} \prec \kappa_{2}$, if there is a measure-preserving map 
$\tau: \mathcal{S}_{1} \rightarrow \mathcal{S}_{2}$ such that for almost every $x \in \mathcal{S}_{1}$, we have

$$
\int_{\tau^{-1}(A)} \kappa_{1}(x, y) d \mu_{1}(y)=\int_{A} \kappa_{2}(\tau(x), y) d \mu_{2}(y)
$$

for all measurable $A \subset \mathcal{S}_{2}$. (This is a very different notion to that appearing in Subsection 2.4, despite the superficial similarity to $\kappa_{1}=\kappa_{2}^{(\tau)}$.) In other words, if we take a particle of $\mathfrak{X}_{\kappa_{1}}$ and look at the distribution of the images under $\tau$ of the types of its children, then this distribution depends only on the image of the type of the original particle, and it does so according to the kernel $\kappa_{2}$. From this description it is immediate that if $\kappa_{1} \prec \kappa_{2}$, then $\pi_{\kappa_{1}}=\pi_{\kappa_{2}}$.

From now on we shall concentrate on the finite-type case, i.e., take $\mathcal{S}$ to be finite. Note that there is a natural correspondence between this case and the case of kernels $\kappa$ on $[0,1]^{2}$ that are piecewise constant on rectangles. In this case $\kappa_{1} \prec \kappa_{2}$ simply means that the types associated to $\kappa_{1}$ may be grouped together to form the types associated to $\kappa_{2}$, and the distribution of the grouped types of the children of a particle in $\mathfrak{X}_{\kappa_{1}}$ is what it should be in $\mathfrak{X}_{\kappa_{2}}$.

The relation $\prec$ is clearly transitive. Hence the natural conjecture is that two kernels give the same distribution on trees if and only if they have a common refinement. Or should it be if and only if they are both refinements of a common 'coarsening'? In fact, somewhat surprisingly, the two are equivalent!

Theorem 6.8. Let $\kappa_{1}$ and $\kappa_{2}$ be finite type kernels. Then the following are equivalent: (i) there is a finite type kernel $\kappa_{\mathrm{r}}$ with $\kappa_{\mathrm{r}} \prec \kappa_{1}$ and $\kappa_{\mathrm{r}} \prec \kappa_{2}$, and (ii) there is a finite type kernel $\kappa_{\mathrm{c}}$ with $\kappa_{1} \prec \kappa_{\mathrm{c}}$ and $\kappa_{2} \prec \kappa_{\mathrm{c}}$.

Proof. Let $\kappa_{i}$ have type-space $\left(\mathcal{S}_{i}, \mu_{i}\right)$. Since the definition of $\prec$ ignores sets of measure zero, we may assume that each $\mu_{i}$ is a strictly positive measure on the finite set $\mathcal{S}_{i}$.

We start by showing that (ii) implies (i). Suppose then that $\kappa_{1} \prec \kappa_{\mathrm{c}}$ and $\kappa_{2} \prec \kappa_{\mathrm{c}}$. Let $\kappa_{\mathrm{c}}$ have type space $\left(\mathcal{S}_{\mathrm{c}}, \mu_{\mathrm{c}}\right)$. Then each of $\mathfrak{X}_{\kappa_{1}}, \mathfrak{X}_{\kappa_{2}}$ may be viewed as $\mathfrak{X}_{\kappa_{\mathrm{c}}}$ with 'extra labels' on the vertices, indicating the subtypes in $\mathcal{S}_{i}$. We wish to add both kinds of label simultaneously. It is easy to write down a way of doing so.

Let $\tau_{i}: \mathcal{S}_{i} \rightarrow \mathcal{S}_{\mathrm{c}}$ be the map witnessing $\kappa_{i} \prec \kappa_{\mathrm{c}}$. Let $\mathcal{S}_{\mathrm{r}}$ be the set of pairs $(i, j) \in \mathcal{S}_{1} \times \mathcal{S}_{2}$ with $\tau_{1}(i)=\tau_{2}(j)$, and set

$$
\mu_{\mathrm{r}}((i, j))=\frac{\mu_{1}(i) \mu_{2}(j)}{\mu_{\mathrm{c}}\left(\tau_{1}(i)\right)} .
$$

Summing first over all $i \in \mathcal{S}_{1}$ and $j \in \mathcal{S}_{2}$ mapping to a given $k \in \mathcal{S}_{c}$, it is easy to check that $\mu_{\mathrm{r}}$ is a probability measure on the finite set $\mathcal{S}_{\mathrm{r}}$. It remains to define an appropriate kernel.

For $(i, j),(k, \ell) \in \mathcal{S}_{\mathrm{r}}$, set

$$
\kappa_{\mathrm{r}}((i, j),(k, \ell))=\frac{\kappa_{1}(i, k) \kappa_{2}(j, \ell)}{\kappa_{\mathrm{c}}\left(\tau_{1}(i), \tau_{1}(k)\right)}
$$


whenever the denominator is non-zero, and set $\kappa_{\mathrm{r}}((i, j),(k, \ell))=0$ otherwise.

Since, despite appearances, the definitions above are symmetric with respect to $\kappa_{1}$ and $\kappa_{2}$, to establish (i) it suffices to show that $\kappa_{\mathrm{r}} \prec \kappa_{1}$. Of course, in doing so we shall map $(i, j) \in \mathcal{S}_{\mathrm{r}}$ to $i \in \mathcal{S}_{1}$. This map is measure preserving. Since $\kappa_{\mathrm{r}}$ is of finite type, all we must check is that for any $(i, j) \in \mathcal{S}_{\mathrm{r}}$ and $k \in \mathcal{S}_{1}$, a particle of type $(i, j)$ in $\mathfrak{X}_{\mathcal{S}_{\mathrm{r}}}$ has the right number of children of (subtypes of) type $k$. In other words, we must show that

$$
\sum_{\ell: \tau_{2}(\ell)=\tau_{1}(k)} \kappa_{\mathrm{r}}((i, j),(k, \ell)) \mu_{\mathrm{r}}((k, \ell))=\kappa_{1}(i, k) \mu_{1}(k) .
$$

If $\kappa_{\mathrm{c}}\left(\tau_{1}(i), \tau_{1}(k)\right)$ is zero then $\kappa_{1}(i, k)$ is also zero (since $\kappa_{1} \prec \kappa_{\mathrm{c}}$ ), so both sides are zero. Otherwise, the left hand side above is simply

$$
\sum_{\ell: \tau_{2}(\ell)=\tau_{1}(k)} \frac{\kappa_{1}(i, k) \kappa_{2}(j, \ell)}{\kappa_{\mathrm{c}}\left(\tau_{1}(i), \tau_{1}(k)\right)} \frac{\mu_{1}(k) \mu_{2}(\ell)}{\mu_{\mathrm{c}}\left(\tau_{1}(k)\right)}=\kappa_{1}(i, k) \mu_{1}(k) \frac{\sum_{\ell: \tau_{2}(\ell)=\tau_{1}(k)} \kappa_{2}(j, \ell) \mu_{2}(\ell)}{\kappa_{\mathrm{c}}\left(\tau_{1}(i), \tau_{1}(k)\right) \mu_{\mathrm{c}}\left(\tau_{1}(k)\right)} .
$$

Recalling that $\tau_{1}(i)=\tau_{2}(j)$, the final fraction is 1 since $\tau_{2}$ is a map witnessing $\kappa_{2} \prec \kappa_{\mathrm{c}}$ : the numerator and denominator both express, for a particle of type $j \in \mathcal{S}_{2}$ (and hence of type $\tau_{2}(j) \in \mathcal{S}_{\mathrm{c}}$ ), the expected number of children of type $\tau_{1}(k)$. This completes the proof that (ii) implies (i).

Suppose now that (i) holds, i.e., that $\kappa_{1}$ and $\kappa_{2}$ have a common refinement $\kappa_{\mathrm{r}}$. Each type in the corresponding type space maps to some $i \in \mathcal{S}_{1}$ and some $j \in \mathcal{S}_{2}$. It is easy to see that we may group together all types mapping to the same pair $(i, j)$, obtaining a refinement with type space $\mathcal{S}_{\text {r }}$ a subset of $\mathcal{S}_{1} \times \mathcal{S}_{2}$. As before, we delete any types with measure zero. We may regard $\mathcal{S}_{\mathrm{r}}$ as the edge set of a bipartite graph $G$ with vertex classes $\mathcal{S}_{1}$ and $\mathcal{S}_{2}$; we shall construct the common coarsening $\kappa_{\mathrm{c}}$ as a kernel with type space the set of components of $G$. Since $\kappa_{\mathrm{r}} \prec \kappa_{1}$, the map from $\mathcal{S}_{\mathrm{r}}$ to $\mathcal{S}_{1}$ given by $(i, j) \mapsto i$ is measure-preserving. In other words, for each $j$, writing $i j$ for $(i, j)$,

$$
\sum_{j: i j \in E(G)} \mu_{\mathrm{r}}(i j)=\mu_{1}(i)
$$

Similarly, $\sum_{i: i j \in E(G)} \mu_{\mathrm{r}}(i j)=\mu_{2}(j)$. For each component $C$ of $G$, set $\mu_{\mathrm{c}}(C)=$ $\sum_{i j \in E(C)} \mu_{\mathrm{r}}(i j)$; this defines a probability measure on the set $\mathcal{S}_{\mathrm{c}}$ of components of $G$. If $C$ has vertex set $C_{1} \cup C_{2}, C_{i} \subset \mathcal{S}_{i}$, then from (64),

$$
\mu_{\mathrm{c}}(C)=\sum_{i \in C_{1}} \sum_{j: i j \in E(G)} \mu_{\mathrm{r}}(i j)=\sum_{i \in C_{1}} \mu_{1}(i) .
$$

Hence the map $\tau_{1}: \mathcal{S}_{1} \rightarrow \mathcal{S}_{\mathrm{c}}$ mapping each $i \in \mathcal{S}_{1} \subset V(G)$ to the component in which it lies is measure preserving. Similarly for the corresponding map $\tau_{2}: \mathcal{S}_{2} \rightarrow \mathcal{S}_{\mathrm{c}}$.

Fix two components $C$ and $C^{\prime}$ of $G$, which need not be distinct. For each edge $e \in C$ set

$$
\lambda\left(e, C^{\prime}\right)=\sum_{f \in E\left(C^{\prime}\right)} \kappa_{\mathrm{r}}(e, f) \mu_{\mathrm{r}}(f),
$$


the expected number of children in $C^{\prime}$ of a particle in $\mathfrak{X}_{\kappa_{\mathrm{r}}}$ of type $e$. Writing $e=i j$, we may rewrite $\lambda\left(e, C^{\prime}\right)$ as follows:

$$
\lambda\left(i j, C^{\prime}\right)=\sum_{k \in C_{1}^{\prime}} \sum_{k \ell \in E(G)} \kappa_{\mathrm{r}}(i j, k \ell) \mu_{\mathrm{r}}(k l)=\sum_{k \in C_{1}^{\prime}} \kappa_{1}(i, k) \mu_{1}(k),
$$

where the second step is from $\kappa_{\mathrm{r}} \prec \kappa_{1}$. This quantity does not depend on $j$, so if $e_{1}, e_{2}$ are edges of $C$ meeting at a vertex of $C_{1}$, then $\lambda\left(e_{1}, C^{\prime}\right)=\lambda\left(e_{2}, C^{\prime}\right)$. A similar argument, using $\kappa_{\mathrm{r}} \prec \kappa_{2}$, gives the same conclusion for edges meeting at a vertex of $C_{2}$. Since $C$ is connected, it follows that $\lambda\left(e, C^{\prime}\right)$ is constant on the edges of $C$. Let us write the common value as $\kappa_{\mathrm{c}}\left(C, C^{\prime}\right) \mu_{\mathrm{c}}\left(C^{\prime}\right)$. Then, for each $i \in C_{1}$,

$$
\sum_{k \in C_{1}^{\prime}} \kappa_{1}(i, k) \mu_{1}(k)=\kappa_{\mathrm{c}}\left(C, C^{\prime}\right) \mu_{\mathrm{c}}\left(C^{\prime}\right),
$$

and similarly, for each $j \in C_{2}$,

$$
\sum_{\ell \in C_{2}^{\prime}} \kappa_{2}(j, \ell) \mu_{2}(\ell)=\kappa_{\mathrm{c}}\left(C, C^{\prime}\right) \mu_{\mathrm{c}}\left(C^{\prime}\right) .
$$

These last two identities establish $\kappa_{1} \prec \kappa_{\mathrm{c}}$ and $\kappa_{2} \prec \kappa_{\mathrm{c}}$ respectively, completing the proof.

The statement of Theorem 6.8 makes sense in the general case, without the restriction to finite-type kernels, but the proof as written does not. It is easy to adapt the proof that (ii) implies (i) to the general case, but it does not seem to be easy to prove that (i) implies (ii) in general. Indeed, it is not impossible that this implication is false in the general case.

Our main aim in this section is to prove the following result.

Theorem 6.9. Let $\kappa_{1}$ and $\kappa_{2}$ be finite-type kernels. Then $\pi\left(\kappa_{1}\right)=\pi\left(\kappa_{2}\right)$ if and only if there is a kernel $\kappa_{\mathrm{c}}$ with $\kappa_{1} \prec \kappa_{\mathrm{c}}$ and $\kappa_{2} \prec \kappa_{\mathrm{c}}$.

The proof will be a little involved (although most of the difficulties are notational rather than actual), so we shall start by illustrating a very simple special case of the basic idea.

Let $\kappa$ be a kernel on the (finite) type space $(\mathcal{S}, \mu)$. In fact, our initial remarks will apply to general kernels. Let $T \in \mathcal{T}^{\mathrm{r}}$ denote a random rooted tree with distribution $\pi(\kappa)$, so $T$ is obtained from $\mathfrak{X}_{\kappa}$ by forgetting the types of the particles. Also, let $T_{t}=\left.T\right|_{t}$ denote the subtree of $T$ corresponding to the first $t$ generations of $\mathfrak{X}_{\kappa}$. We shall show that the distribution of $T_{t}$ is determined by a certain function of $\kappa$, and vice versa. We start by writing out the much simpler case $t=1$.

The tree $T_{1}$ is simply a star, so its distribution is determined by the distribution of the degree of the root, i.e., the distribution of the number $d_{0}$ of children of the initial particle of $\mathfrak{X}_{\kappa}$. As in [10, for each $x \in \mathcal{S}$, let us write

$$
\lambda(x)=\lambda_{1}(x)=\int_{\mathcal{S}} \kappa(x, y) d \mu(y)
$$


for the expected degree of $x$, i.e., the expected number of children of a particle of type $x$. (The reason for the subscript 1 will become clear later.) Also, let $\Lambda=\Lambda_{1}$ denote the (first order) expected degree distribution of $\kappa$, i.e., the distribution of $\lambda(x)$ when $x$ is chosen according to the distribution $\mu$. From the definition of $\mathfrak{X}_{\kappa}$, given the type of the root, $d_{0}$ has a Poisson distribution with mean $\lambda(x)$. Thus the unconditional distribution of $d_{0}$ is the mixed Poisson distribution $\operatorname{Po}(\Lambda)$, defined in the discrete case by

$$
\mathbb{P}(\operatorname{Po}(\Lambda)=k)=\sum_{\lambda} \mathbb{P}_{\Lambda}(\lambda) \frac{\lambda^{k} e^{-\lambda}}{k !},
$$

where the sum is over the finite set of possible values of $\Lambda$. It follows that the distribution $\Lambda$ determines the distribution of $d_{0}$, and hence of $T_{1}$. The reverse is also true, since the distribution of $\operatorname{Po}(\Lambda)$ determines that of $\Lambda$. (This is trivial in the discrete case, using the tail of $\mathbb{P}(\operatorname{Po}(\Lambda)=k)$ for large $k$ to identify the maximum possible value of $\Lambda$ and its probability, then subtracting off the corresponding contribution to the sum in (65), identifying the next largest possible value, and so on. The general case is not hard, using the generating function of the distribution $\operatorname{Po}(\Lambda)$.)

Let $x$ be a type with $\lambda(x)>0$. From the definition of $\mathfrak{X}_{\kappa}$, the types of the children of a particle of type $x$ form a Poisson process on $\mathcal{S}$ with intensity measure $\mu_{x}$, defined by $d \mu_{x}(y)=\kappa(x, y) d \mu(y)$. In order to understand the distribution of $T_{2}$, we consider the offspring expected degree distribution $\lambda_{2}(x)$, the image of $\mu_{x}(y)$ under the map $y \mapsto \lambda_{1}(y)$. Thus, if $\mu_{x}$ were a probability measure, $\lambda_{2}(x)$ would be the distribution of $\lambda_{1}(Y)$ when $Y$ has the distribution $\mu_{x}$; in general, neither $\mu_{x}$ nor $\lambda_{2}(x)$ is a probability measure: they both have total mass $\mu_{x}(\mathcal{S})=\lambda_{1}(x)$.

Similarly, for $k \geq 3$, we define $\lambda_{k}(x)$ to be the image of the measure $\mu_{x}(y)$ under the map $y \mapsto \lambda_{k-1}(y)$. Thus

$$
\left(\lambda_{k}(x)\right)(A)=\int_{y \in \mathcal{S}: \lambda_{k-1}(y) \in A} d \mu_{x}(y)=\int_{y \in \mathcal{S}: \lambda_{k-1}(y) \in A} \kappa(x, y) d \mu(y) .
$$

Note that for a given $x, \lambda_{1}(x)=\lambda(x)$ is a real number, $\lambda_{2}(x)$ is a measure on the reals, $\lambda_{3}(x)$ is a measure on the set of measures on the reals, and so on. If $\kappa$ is of finite type, then all these measures are discrete. By the $k$-th order expected degree distribution $\Lambda_{\kappa}$ of $\kappa$, we mean the distribution of $\lambda_{k}(x)$ when $x$ is chosen randomly with distribution $\mu$.

We shall deduce Theorem 6.9 from the following lemma.

Lemma 6.10. Fix $k \geq 1$, and let $\kappa$ be a finite-type kernel. Then the distribution $\Lambda_{k}$ determines the distribution of $T_{k}$ and vice versa.

The restriction to finite-type kernels is presumably not needed here, but simplifies the proofs, avoiding any possible difficulties associated to choosing the right notion of convergence. Note that we have already proved the case $k=1$.

Before proving Lemma 6.10, let us show that Theorem 6.9 does indeed follow. 
Proof of Theorem 6.9. If $\kappa_{1} \prec \kappa_{\mathrm{c}}$ and $\kappa_{2} \prec \kappa_{\mathrm{c}}$, then $\pi\left(\kappa_{1}\right)=\pi\left(\kappa_{\mathrm{c}}\right)=\pi\left(\kappa_{2}\right)$; the content of the theorem is the reverse implication. We shall show that if $\kappa$ is a finite-type kernel, then one can define a (finite type) kernel $\kappa_{\mathrm{c}}$ with $\kappa \prec \kappa_{\mathrm{c}}$ in a way that depends only on $\pi(\kappa)$; this clearly implies the result.

Given a finite type kernel $\kappa$ on $(\mathcal{S}, \mu)$, define an equivalence relation $\sim$ on $\mathcal{S}$ by $x \sim y$ if $\lambda_{k}(x)=\lambda_{k}(y)$ for every $k$. If $x \not y$, then there is some smallest $k=$ $k(x, y)$ such that $\lambda_{k}(x) \neq \lambda_{k}(y)$. Let $K$ be an upper bound on the set $\{k(x, y)$ : $x, y \in \mathcal{S}, x \nsim y\}$, which exists since $\mathcal{S}$ is finite. Since $\lambda_{k+1}(x)$ determines $\lambda_{k}(x)$, we have $\lambda_{K}(x) \neq \lambda_{K}(y)$ whenever $x \nsim y$, so

$$
x \sim y \Longleftrightarrow \lambda_{K}(x)=\lambda_{K}(y) \Longleftrightarrow \lambda_{K+1}(x)=\lambda_{K+1}(y) .
$$

Note that $K$ is determined by the set $\Lambda_{k}, k=0,1,2, \ldots$ : we may take $K$ to be the smallest integer such that the distribution of $\lambda_{K+1}$ (which then determines that of $\lambda_{K}$ ) has property (66).

Let us define a type space $\mathcal{S}_{\mathrm{c}}$ whose elements are the possible values of $\lambda_{K}(x)$, $x \in \mathcal{S}$, and a corresponding $\mu_{\mathrm{c}}$ given by the $\mu$-probability of the corresponding set of (equivalent) types $x \in \mathcal{S}$. Note that $\mathcal{S}_{\mathrm{c}}$ and $\mu_{\mathrm{c}}$ depend only on the distribution of $\lambda_{K}(x)$, and hence are determined by $\Lambda_{K+1}$. We map $\mathcal{S}$ to $\mathcal{S}_{\mathrm{c}}$ in the obvious way; this map is measure preserving by definition. The distribution of $\lambda_{K}(y)$ over children $y$ of a particle of type $x$ is determined by $\lambda_{K+1}(x)$, and hence, from (66), by $\lambda_{K}(x)$. It follows that the distribution of the $\mathcal{S}_{\mathrm{c}}$-types of the children depends only on the $\mathcal{S}_{\mathrm{c}}$-type of $x$. This is exactly the statement that there is a kernel $\kappa_{\mathrm{c}}$ on $\left(\mathcal{S}_{\mathrm{c}}, \mu_{\mathrm{c}}\right)$ with $\kappa \prec \kappa_{\mathrm{c}}$. The kernel $\kappa_{\mathrm{c}}$ is determined by the distribution of $\Lambda_{K+1}(x)$, i.e., by $\Lambda_{K+2}$. Hence, $\kappa_{\mathrm{c}}$ is determined by the sequence $\Lambda_{k}, k=1,2, \ldots$ Using Lemma 6.10 it follows that $\kappa_{\mathrm{c}}$ is determined by the distribution of $T_{k}$ for all $k$, and thus by $\pi(\kappa)$. As noted above the existence of such a $\kappa_{\mathrm{c}}$ with $\kappa \prec \kappa_{\mathrm{c}}$ suffices to prove the theorem.

It remains to prove Lemma 6.10. Note the lemma makes two separate statements; in proving Theorem 6.9 we only used one of these, that the distribution of $T_{k}$ determines that of $\Lambda_{k}$. We shall prove Lemma 6.10 by induction; for this we need both statements. In fact, to make the induction work, we shall need to prove a little more.

Let $\kappa$ be a kernel on the finite type-space $(\mathcal{S}, \mu)$. The measure $\mu$ plays two roles in the branching process $\mathfrak{X}_{\kappa}$ : it appears in the distribution of the offspring of a particle, and also in the distribution of the type of the initial particle. It will be convenient to generalize $\mathfrak{X}_{\kappa}$ slightly as follows: let $\mu_{0}$ be any probability measure on $\mathcal{S}$, and let $\mathfrak{X}_{\kappa}\left(\mu_{0}\right)$ be the branching process defined as $\mathfrak{X}_{\kappa}$, but starting with a single particle of type distributed according to $\mu_{0}$. Note that $\mathfrak{X}_{\kappa}\left(\mu_{0}\right)$ depends on $\mu$ as well as $\mu_{0}$, and that $\mathfrak{X}_{\kappa}(\mu)=\mathfrak{X}_{\kappa}$.

Let $\Lambda_{k}\left(\mu_{0}\right)$ denote the distribution of $\lambda_{k}(x)$ when $x$ is chosen randomly with distribution $\mu_{0}$, so $\Lambda_{k}(\mu)=\Lambda_{k}$. Also, let $T_{k}\left(\mu_{0}\right)$ denote the random rooted tree obtained from the first $k$ generations of $\mathfrak{X}_{\kappa}\left(\mu_{0}\right)$ by forgetting the types of the particles. The following lemma is slightly stronger than Lemma 6.10, which can be recovered by setting $\mu_{0}=\mu$. 
Lemma 6.11. Fix $k \geq 1$, let $\kappa$ be a finite-type kernel on $(\mathcal{S}, \mu)$, and let $\mu_{0}$ be a probability measure on $\mathcal{S}$. Then (i) the distribution $\Lambda_{k}\left(\mu_{0}\right)$ determines the distribution of $T_{k}\left(\mu_{0}\right)$, and (ii) the distribution of $T_{k}\left(\mu_{0}\right)$ determines $\Lambda_{k}\left(\mu_{0}\right)$.

Proof. We start with the easier statement, namely part (i), which we prove by induction on $k$; the case $k=1$ is trivial, as noted above: $T_{1}$ is a star the degree of whose root has the mixed Poisson distribution $\operatorname{Po}\left(\Lambda_{1}\left(\mu_{0}\right)\right)$.

Suppose then that $k \geq 2$ and that (i) holds with $k$ replaced by $k-1$. It is easy to see that it suffices to prove (i) with $\mu_{0}$ concentrated on a single type $x$, in which case $\Lambda_{k}\left(\mu_{0}\right)=\lambda_{k}(x)$. Let us fix the type $x \in \mathcal{S}$ of the root, writing $\mathfrak{X}_{\kappa}(x)=\mathfrak{X}_{\kappa}\left(\delta_{x}\right)$ for the branching process $\mathfrak{X}_{\kappa}$ started with a single particle of type $x$.

Let $X_{1}$ denote the first generation of $\mathfrak{X}_{\kappa}(x)$. Given $X_{1}$, the descendants of a particle $v$ in $X_{1}$ have the distribution of $\mathfrak{X}_{\kappa}(y)$, where $y$ is the type of $v$, and the subtrees corresponding to different $v$ are independent. By induction, the distribution of the first $k-1$ generations of the descendants of $v$ are determined by $\lambda_{k-1}(y)$. Hence, given $X_{1}$, the conditional distribution of $T_{k}$ depends only on the multiset $M=\left\{\lambda_{k-1}(y)\right\}$, where $y$ runs over the types in $X_{1}$. Given the type $x$ of the root, the types of the particles in $X_{1}$ form a Poisson process on $\mathcal{S}$ with intensity measure $\mu_{x}$. Hence, $M$ is a Poisson process on the appropriate space of distributions with intensity measure $\lambda_{k}(x)$. In particular, the distribution of $M$, and hence that of $T_{k}$, is determined by $\lambda_{k}(x)$, completing the proof of part (i) by induction.

We now turn to the reverse implication, part (ii), which we again prove by induction. For $k=1$ the argument is as before: the degree of the root in $T_{1}\left(\mu_{0}\right)$ has a mixed Poisson distribution $\operatorname{Po}\left(\Lambda_{1}\left(\mu_{0}\right)\right)$, which determines $\Lambda_{1}\left(\mu_{0}\right)$. Suppose then that $k \geq 2$, and that (ii) holds with $k$ replaced by $k-1$, i.e., that for any probability measure $\mu_{0}^{\prime}$ on $\mathcal{S}$, the distribution of $T_{k-1}\left(\mu_{0}^{\prime}\right)$ determines $\Lambda_{k-1}\left(\mu_{0}^{\prime}\right)$. This time we cannot simply condition on the type of the root, as we are given the distribution of $T_{k}$ without types. It is here that we shall use the fact that $\mathcal{S}$ is finite.

For any realization $T$ of the random tree $T_{k}\left(\mu_{0}\right)$, let $\mathcal{D}(T)$ denote the empirical distribution of the branches of $T$, i.e., the subtrees of $T$ of height $k-1$ rooted at the neighbours of the root of $T$. Thus $\mathcal{D}(T)$ is a distribution on $\mathcal{T}_{k-1}^{\mathrm{r}}$, the set of rooted trees of height $k-1$, and the weight $\mathcal{D}(T)$ assigns to a tree $T^{\prime}$ is just the number of branches of $T$ that are isomorphic to $T^{\prime}$ divided by the total number of branches. Let $\tau_{0}$ denote the type of the root of $\mathfrak{X}_{\kappa}\left(\mu_{0}\right)$, and $d_{0}=\left|X_{1}\right|$ its degree. Given that $\tau_{0}=x$ and $d_{0}=N$, the types of the particles in $X_{1}$ are independent with the distribution $\widetilde{\mu}_{x}=\mu_{x} / \mu_{x}(\mathcal{S})=\mu_{x} / \lambda(x)$, the normalized version of $\mu_{x}$. Hence their descendants are independent copies of the branching process $\mathfrak{X}_{\kappa}\left(\widetilde{\mu}_{x}\right)$, and the corresponding branches are $N$ independent samples from the distribution $T_{k-1}\left(\widetilde{\mu}_{x}\right)$. Let us view the empirical distribution $\mathcal{D}\left(T_{k}\left(\mu_{0}\right)\right)$ as a point $\mathbf{v}$ in the space $[0,1]^{\mathcal{T}_{k-1}^{\mathrm{r}}}$ taken with the supremum norm. This point is random, since it depends on the realization of $\mathfrak{X}_{\kappa}\left(\mu_{0}\right)$. From the law of large numbers and the comments above, as $N \rightarrow \infty$ the random point $\mathbf{v}$ obtained after conditioning on $\tau_{0}=x$ and $d_{0}=N$ converges in probability to 
the single point $T_{k-1}\left(\widetilde{\mu}_{x}\right) \in[0,1]^{\mathcal{T}_{k-1}^{\mathrm{r}}}$.

Since all we are given is the distribution of $T_{k}\left(\mu_{0}\right)$, we cannot condition on the type of the root. We can however condition on its degree, $d_{0}=\left|X_{1}\right|$. Let $\lambda_{\max }$ be the largest value of $\lambda(x)$ for $x \in \mathcal{S}$ with $\mu_{0}(x)>0$. As $N \rightarrow \infty$,

$$
\mathbb{P}\left(\tau_{0}=x \mid d_{0}=N\right) \rightarrow p_{x}=\mu_{0}(x) / \mu_{0}\left(\left\{y: \lambda(y)=\lambda_{\max }\right\}\right)
$$

if $\lambda(x)=\lambda_{\max }$, and to zero otherwise. From the comments above, as $N \rightarrow \infty$, the distribution of $\mathbf{v}=\mathcal{D}\left(T_{k}\left(\mu_{0}\right)\right)$ given that $d_{0}=N$ tends to the discrete distribution taking each value $T_{k-1}\left(\widetilde{\mu}_{x}\right)$ with probability $p_{x}$. Hence, the distribution $T_{k}\left(\mu_{0}\right)$ determines what distributions are possible for $T_{k-1}\left(\widetilde{\mu}_{x}\right)$, and the probability of each (a sum of $p_{x^{\prime}}$ over $x^{\prime}$ such that $T_{k-1}\left(\widetilde{\mu}_{x^{\prime}}\right)=T_{k-1}\left(\widetilde{\mu}_{x}\right)$ ).

By induction, for each possible distribution $T_{k-1}\left(\widetilde{\mu}_{x}\right)$ there is a unique corresponding distribution $\Lambda_{k-1}\left(\widetilde{\mu}_{x}\right)$. Since we know $\lambda_{\max }$, and the measure $\Lambda_{k-1}\left(\mu_{x}\right)$ is simply obtained by multiplying the probability measure $\Lambda_{k-1}\left(\widetilde{\mu}_{x}\right)$ by the constant factor $\lambda_{\max }$, we recover the possible distributions $\Lambda_{k-1}\left(\mu_{x}\right)$ for $x$ with $\lambda(x)=\lambda_{\max }$, and the $p$-probability of each. In other words, we recover the distribution $\Lambda_{k}(p)$ where $p$ is the probability measure defined by $p(\{x\})=p_{x}$.

By part (i), the distribution $\Lambda_{k}(p)$ determines the distribution of $T_{k}(p)$, which is simply that of $T_{k}\left(\mu_{0}\right)$ conditioned on $\tau_{0}$ lying in the set $\lambda\left(\tau_{0}\right)=\lambda_{\max }$. Since we recover this distribution exactly, we also recover the conditional distribution of $T_{k}\left(\mu_{0}\right)$ given that $\lambda\left(\tau_{0}\right)<\lambda_{\max }$, i.e., we recover the distribution $T_{k}\left(\mu_{0}^{\prime}\right)$, where $\mu_{0}^{\prime}$ is the distribution $\mu_{0}$ conditioned on $\lambda(\cdot)<\lambda_{\max }$. Repeating the argument above, we can recover the part of $\Lambda_{k}\left(\mu_{0}^{\prime}\right)$ coming from the largest possible $\lambda$-value of the root, i.e., the part of $\Lambda_{k}\left(\mu_{0}\right)$ coming form the second largest value, and so on. This shows that the distribution of $T_{k}\left(\mu_{0}\right)$ does determine that of $\Lambda_{k}\left(\mu_{0}\right)$, completing the proof of (ii) by induction.

Theorem 6.9 shows that there are many examples of different kernels that give rise to the same branching process, and hence to the same distribution of tree counts in the corresponding random graphs $G_{1 / n}(n, \kappa)$. One extremely special case concerns homogeneous kernels: we say that $\kappa$ is homogeneous with degree $c$ if $\int_{y} \kappa(x, y) d y=c$ for almost every $x$. In this case, $\mathfrak{X}_{\kappa}$ seen without types becomes a standard single type Galton-Watson branching process $\mathfrak{X}_{c}$ in which each particle has a Poisson number of children with mean $c$. Writing $c$ also for the constant kernel taking the value $c$, Theorem 6.9 shows that $\pi(\kappa)=\pi(c)$ if and only if $\kappa$ is homogeneous with degree $c$. (This special case is essentially trivial, however: one need consider only the first generation of the branching process.)

In the denser case, i.e., whenever $n p \rightarrow \infty$, Lemma 4.10 tells us that the sequence $G_{p}(n, \kappa)$ converges to $\kappa$ in the cut metric with probability 1 , so it follows that the models $G_{p}\left(n, \kappa_{1}\right)$ and $G_{p}\left(n, \kappa_{2}\right)$ are genuinely different unless $\kappa_{1} \sim \kappa_{2}$ in the sense of Subsection 2.4 in which case they are trivially the same. This suggests that we need better methods of distinguishing very sparse graphs than looking at tree counts. However, as we shall see in this next sections, while this is true, the question of which pairs of kernels give rise to equivalent models is not so easy. 


\subsection{The partition metric}

In the spirit of the rest of the paper, we say that two graphs with $n$ vertices are essentially the same if one can be changed into a graph isomorphic to the other by adding and deleting $o\left(p n^{2}\right)$ edges. (Of course, the definition makes formal sense only for two sequences.) Otherwise, they are essentially different. In all previous sections, graphs that were essentially the same were treated as equivalent, in the sense that their distance in any of the metrics we considered tends to zero.

Let $p=1 / n$, and let $\kappa$ be a kernel whose corresponding branching process always dies out. In the notation of Bollobás, Janson and Riordan [10], we assume that the operator $T_{\kappa}$ corresponding to the kernel $\kappa$ satisfies $\left\|T_{\kappa}\right\| \leq 1$, i.e., $\kappa$ is (weakly) subcritical. From the results in [10, almost all vertices of $G_{1 / n}(n, \kappa)$ are in small tree components: more precisely, given any $\varepsilon>0$, there is a $K$ such that, whp, all but at most $\varepsilon n$ vertices of $G_{1 / n}(n, \kappa)$ are in tree components with size at most $K$. Furthermore, the asymptotic number of copies of a given tree $T$ in $G_{1 / n}(n, \kappa)$ is determined by the probability of $T$ in the distribution $\pi(\kappa)$. It follows that if $\kappa_{1}$ and $\kappa_{2}$ are subcritical kernels, then $G_{1 / n}\left(n, \kappa_{1}\right)$ and $G_{1 / n}\left(n, \kappa_{2}\right)$ are (whp) essentially the same if and only if $\pi\left(\kappa_{1}\right)=\pi\left(\kappa_{2}\right)$. Hence, in the subcritical case, the random graph $G_{1 / n}(n, \kappa)$ depends only on the branching process $\mathfrak{X}_{\kappa}$. Of course, this rather trivial observation does not extend to the supercritical case.

Given two real numbers $a, b \geq 0$, let $\kappa_{a, b}$ denote the 2 -by-2 'chessboard' kernel defined as follows:

$$
\kappa_{a, b}(x, y)= \begin{cases}a & \text { if } x<1 / 2, y<1 / 2 \text { or } x \geq 1 / 2, y \geq 1 / 2 \\ b & \text { otherwise }\end{cases}
$$

To form the random graph $G_{p}\left(n, \kappa_{a, b}\right)$, we partition the vertex set randomly into two parts, and then take each cross-edge to be present with probability $b p$, and each other edge with probability ap. Note that $\kappa_{a, b}$ is homogeneous with constant $(a+b) / 2$. Also, if $a=b$, then $\kappa_{a, b}$ is simply the constant kernel taking the value $a=b$.

For $p=1 / n$, perhaps the simplest example of two sequences of essentially different graphs not distinguished by the tree counts is given by the random graphs $G_{1 / n}\left(n, \kappa_{2,2}\right)$ and $G_{1 / n}\left(n, \kappa_{4,0}\right)$, i.e., the usual Erdős-Rényi random graph $G(n, 2 / n)$ and (essentially) the random bipartite graph $G(n / 2, n / 2 ; 4 / n)$. How do we know that these graphs are different? For the obvious reason that one is bipartite, with almost equal vertex classes, while the other is not. Indeed, the smallest balanced cut in $G(n, 2 / n)$ has size of order $\Theta(n)$ : this follows, for example, from the result of Luczak and McDiarmid [37] that removing $o(n)$ edges from the giant component of $G(n, c / n), c>1$, leaves a connected component with only $o(n)$ fewer vertices than the original giant. Note that one has to be a little careful here: writing $\rho(c)$ for the largest solution to $\rho=1-e^{-c \rho}$, so $\rho(c) n$ is the typical size of the giant component in $G(n, c / n)$, we need $\rho>1 / 2$; otherwise, it is easy to construct a balanced cut with $o(n)$ edges across it. Note that both $G(n, 2 / n)$ and $G(n / 2, n / 2 ; 4 / n)$ have balanced cuts with a range of 
sizes: the difference between the two graphs can be seen in the difference between these ranges.

In the discussion above we considered balanced partitions of the vertex set of a graph into two pieces. Of course, it makes sense to consider any fixed number $k$ of pieces. Let $p=p(n)$ be a normalizing function as usual, and $G_{n}$ a graph with $n$ vertices. Let $k \geq 2$ be fixed. For $n \geq k$ and $\Pi=\left(P_{1}, \ldots, P_{k}\right)$ a partition of $V\left(G_{n}\right)$ into $k$ non-empty parts, let $M_{\Pi}\left(G_{n}\right)$ be the $k$-by- $k$ matrix whose $i j$ th entry is $d_{p}\left(P_{i}, P_{j}\right)$, the normalized density of edges from $P_{i}$ to $P_{j}$. Since $M_{\Pi}\left(G_{n}\right)$ is symmetric, we may think of this matrix as an element of $\mathbb{R}^{k(k+1) / 2}$. Set

$$
\mathcal{M}_{k}\left(G_{n}\right)=\left\{M_{\Pi}\left(G_{n}\right)\right\} \subset \mathbb{R}^{k(k+1) / 2},
$$

where $\Pi$ runs over all balanced partitions of $V\left(G_{n}\right)$ into $k$ parts, i.e., all partitions $\left(P_{1}, \ldots, P_{k}\right)$ with $\left|P_{i}-P_{j}\right| \leq 1$.

As usual, we always assume that $G_{n}$ has $O\left(p n^{2}\right)$ edges. For definiteness, let us assume that $e\left(G_{n}\right) \leq M p n^{2}$. Since each part of a balanced partition has size at least $n /(2 k)$, the entries of any $M_{\Pi}\left(G_{n}\right) \in \mathcal{M}_{k}\left(G_{n}\right)$ are bounded by $M_{k}=(2 k)^{2} M$, say. Thus, $\mathcal{M}_{k}\left(G_{n}\right)$ is a subset of the compact space $\mathcal{M}_{k}=$ $\left[0, M_{k}\right]^{k(k+1) / 2}$.

Let $\mathcal{C}\left(\mathcal{M}_{k}\right)$ denote the set of non-empty compact subsets of $\mathcal{M}_{k}$, and let $d_{\mathrm{H}}$ be the Hausdorff metric on $\mathcal{C}\left(\mathcal{M}_{k}\right)$, defined with respect to the $\ell_{\infty}$ distance, say. Note that $\left(\mathcal{C}\left(\mathcal{M}_{k}\right), d_{\mathrm{H}}\right)$ is compact, since $\mathcal{M}_{k}$ is compact. To ensure that the metric we are about to define is a genuine metric, it is convenient to add the empty set to $\mathcal{C}\left(\mathcal{M}_{k}\right)$, extending $d_{\mathrm{H}}$ so that the empty set is an isolated point.

Let $\mathcal{C}=\prod_{k \geq 2} \mathcal{C}\left(\mathcal{M}_{k}\right)$, and let $\mathcal{M}: \mathcal{F} \mapsto \mathcal{C}$ be the map defined by

$$
\mathcal{M}\left(G_{n}\right)=\left(\mathcal{M}_{k}\left(G_{n}\right)\right)_{k=2}^{\infty}
$$

for every graph $G_{n}$ on $n$ vertices, noting that $\mathcal{M}_{k}\left(G_{n}\right)$ is empty if $k>n$. Then we may define the partition metric $d_{\text {part }}$ by

$$
d_{\text {part }}\left(G, G^{\prime}\right)=d\left(\mathcal{M}(G), \mathcal{M}\left(G^{\prime}\right)\right),
$$

where $d$ is any metric on $\mathcal{C}$ giving rise to the product topology. Considering the partition of an $n$ vertex graph into $n$ parts shows that $d_{\text {part }}$ is a metric on the set $\mathcal{F}$ of isomorphism classes of finite graphs. Recalling that each space $\left(\mathcal{C}\left(\mathcal{M}_{k}\right), d_{\mathrm{H}}\right)$ is compact, the key property of the partition metric is that $\left(G_{n}\right)$ is Cauchy with respect to $d_{\text {part }}$ if and only if there are compact sets $Y_{k} \subset \mathcal{M}_{k}$ such that $d_{\mathrm{H}}\left(\mathcal{M}_{k}\left(G_{n}\right), Y_{k}\right) \rightarrow 0$ for each $k$. In particular, convergence in $d_{\text {part }}$ is equivalent to convergence of the set of partition matrices for each fixed $k$. Thus we may always think of $k$ as fixed and $n$ as much larger than $k$.

In the dense case, a metric equivalent to $d_{\text {part }}$ has been introduced independently by Borgs, Chayes, Lovász, Sós and Vesztergombi [18]; the only difference is that in [18, all partitions into $k$ parts are considered, rather than just balanced partitions. Of course, one then needs to take care to ensure that the densities between small parts are counted with an appropriate weight when computing the distance between density matrices $\mathcal{M}_{k}$. Whether one takes all 
partitions or just balanced partitions is a matter of taste: it is very easy to see that convergence in either of the resulting metrics implies convergence in the other.

The definitions above may appear rather unnatural: the set $\mathcal{M}_{k}\left(G_{n}\right)$ of possible density matrices is perhaps more naturally seen as a multiset, with one element for each of the $N_{n, k}$ balanced partitions of $[n]$ into $k$ (ordered) parts; the Hausdorff metric ignores the multiplicities of the points of these sets. For multisets $S, S^{\prime}$ in a metric space $(X, d)$ with $|S|=\left|S^{\prime}\right|=N$, (a version of) their matching distance is given by

$$
d_{\text {match }}\left(S, S^{\prime}\right)=\min _{\phi} \max _{x \in S} d(x, \phi(x)),
$$

where $\phi$ runs over all bijections between $S$ and $S^{\prime}$ (as multisets). In other words, we pair up the points of $S$ with those of $S^{\prime}$ and measure the maximum distance between corresponding points, minimized over pairings. For graphs $G_{n}, G_{n}^{\prime}$ with the same number of vertices, it may make sense to use $d_{\text {match }}$ instead of $d_{\mathrm{H}}$ to measure the distance between $\mathcal{M}_{k}\left(G_{n}\right)$ and $\mathcal{M}_{k}\left(G_{n}^{\prime}\right)$, defining the partition matching distance $d_{\mathrm{p}-\mathrm{m}}\left(G_{n}, G_{n}^{\prime}\right)$ accordingly.

In fact, $d_{\text {match }}$ can easily be extended to multisets $S, S^{\prime}$ with different numbers of elements: simply replace each point of $S$ by $\left|S^{\prime}\right|$ points, and each point of $S^{\prime}$ by $|S|$ points, then compute the matching distance. In other words, minimize over 'fractional bijections' $\phi$ above; if $|S|=\left|S^{\prime}\right|$ this does not change the minimum distance. Extending $d_{\text {match }}$ in this way, we could thus define $d_{\mathrm{p}-\mathrm{m}}\left(G, G^{\prime}\right)$ for any two graphs $G, G^{\prime}$. However, the resulting metric behaves much less well than $d_{\text {part }}$ : for example, not all (sparse) sequences will have subsequences that are Cauchy, in contrast to $d_{\text {part }}$, which, as noted above, is defined via a metric on a compact space. Even worse, it is easy to see that the sequence $(G(n, c / n))$ will not converge in the partition matching metric.

The matching distance and the Hausdorff distance share what might appear to be an undesired property: they are strongly influenced by atypical partitions П. Surely, for multisets, it would be more natural to weight points by their multiplicity, replacing (68) by

$$
d_{\text {match }}^{\prime}\left(S, S^{\prime}\right)=\min _{\phi} \frac{1}{N} \sum_{x \in S} d(x, \phi(x)) .
$$

Recall, however, that our main aim in introducing the partition metric was to distinguish in a sensible way such pairs of graphs as the uniform random graph $G_{n}=G(n, 2 / n)$ and the random bipartite graph $G_{n}^{\prime}=G(n / 2, n / 2 ; 4 / n)$. It is very easy to see that for almost all partitions of $V\left(G_{n}^{\prime}\right)$ into a fixed number $k$ of parts, each part contains almost equal numbers of vertices from the two vertex classes of $G_{n}^{\prime}$. It follows that almost all (in the multiset sense) density matrices arising from $G_{n}^{\prime}$ are very close to each other and to almost all density matrices arising from $G_{n}$. The whole point of the partition metric is that it accords significant weight to atypical partitions, in particular, to the partition of $G_{n}^{\prime}$ corresponding to its two vertex classes. For this reason we now return to 
considering $\mathcal{M}_{k}\left(G_{n}\right)$ as a set rather than a multiset, and stay with the definition of $d_{\text {part }}$ above based on the Hausdorff metric.

We may extend $\mathcal{M}$, and hence $d_{\text {part }}$, to kernels in a natural way: instead of partitioning the vertex set into $k$ almost equal parts, we partition $[0,1]$ into $k$ exactly equal parts; we omit the details. There is a slight caveat here: it is not clear that $\mathcal{M}_{k}(\kappa)$ is a closed set. However, this is irrelevant: we simply take its closure. (In fact, as shown by Borgs, Chayes, Lovász, Sós and Vesztergombi 18, $\mathcal{M}_{k}(\kappa)$ is not in general closed; see their Example 4.4. They consider more general 'fractional partitions', showing that the set of density matrices obtained from these is the closure of $\mathcal{M}_{k}(\kappa)$.)

As for the cut metric, it is easy to check that it makes little difference whether we define $d_{\text {part }}$ for graphs directly, or by going via kernels. (The corresponding result appears in [18: the sparse case here is slightly more complicated due to the possibility of 'high-degree' vertices.)

Lemma 6.12. Let $p=p(n)$ satisfy $p \geq 1 / n$, and let $G_{n}$ be a sequence of graphs with $e\left(G_{n}\right)=O\left(p n^{2}\right)$ and $\Delta\left(G_{n}\right)=o\left(p n^{2}\right)$. Then, for each $k$,

$$
d_{\mathrm{H}}\left(\mathcal{M}_{k}\left(G_{n}\right), \mathcal{M}_{k}\left(\kappa_{G_{n}}\right)\right) \rightarrow 0
$$

as $n \rightarrow \infty$.

Proof. Since $e\left(G_{n}\right)=O\left(p n^{2}\right)$, there is a constant $D$ such that at most $n /(2 k)$ vertices of $G_{n}$ have degree more than $D p n$. Let $L$ denote the set of 'low-degree' vertices, with degree at most $D p n$, so $|L| \geq n-n /(2 k)$.

We must show that for any density matrix in $\mathcal{M}_{k}\left(G_{n}\right)$ there is a nearby matrix in $\mathcal{M}_{k}\left(\kappa_{G_{n}}\right)$, and vice versa. The forward implication is trivial: a balanced partition $\Pi$ of $V\left(G_{n}\right)$ corresponds to a partition of $[0,1]$ into sets whose sizes differ by $O(1 / n)=o(1)$. Adjusting these parts slightly, making changes only in subintervals of $[0,1]$ corresponding to low degree vertices, the entries of the corresponding density matrix change by $o(1)$.

For the reverse implication, let $\Pi$ be a partition of $[0,1]$ into $k$ parts $P_{1}, \ldots, P_{k}$, and let $M \in \mathcal{M}_{k}\left(\kappa_{G_{n}}\right)$ be the corresponding density matrix, with entries $m_{i j}$. For $v \in V\left(G_{n}\right)=[n]$ and $1 \leq i \leq k$, let $p_{v, i}$ be the fraction of the subinterval of $[0,1]$ corresponding to the vertex $v$ that lies in $P_{i}$, noting that $\sum_{i} p_{v, i}=1$ for each $v$, and $\sum_{v} p_{v, i}=n / k$ for each $i$. Form a random partition $\Pi^{\prime}=\left(P_{1}^{\prime}, \ldots, P_{k}^{\prime}\right)$ as follows: put each vertex $v$ into a random part $P_{i_{v}}^{\prime}$ with $\mathbb{P}\left(i_{v}=i\right)=p_{v, i}$, with the choices independent for different vertices.

It is immediate that $\mathbb{E}\left(\left|P_{i}^{\prime}\right|\right)=n / k$ and $\operatorname{Var}\left(\left|P_{i}^{\prime}\right|\right) \leq n / k$. It follows that for some constant $C$ we have

$$
\forall i:|| P_{i}^{\prime}|-n / k| \leq C \sqrt{n}
$$

with probability at least $99 \%$. Writing $v \sim w$ if $v w \in E\left(G_{n}\right)$, for $1 \leq i, j \leq k$ 
we have

$$
\begin{aligned}
\mathbb{E}\left(e\left(P_{i}^{\prime}, P_{j}^{\prime}\right)\right)=\sum_{(v, w): v \sim w} \mathbb{E}\left(1_{i_{v}=i} 1_{i_{w}=j}\right)=\sum_{(v, w): v \sim w} p_{v, i} p_{w, j} \\
\\
=n^{2} p \int_{P_{i} \times P_{j}} \kappa_{G_{n}}(x, y) d x d y,
\end{aligned}
$$

so the expectation of $e\left(P_{i}^{\prime}, P_{j}^{\prime}\right) /\left(n^{2} p\right)$ is exactly $m_{i j} / k^{2}$. For edges $v w, v^{\prime} w^{\prime}$ of $G_{n}$, the random variables $1_{i_{v}=i} 1_{i_{w}=j}$ and $1_{i_{v^{\prime}}=i} 1_{i_{w^{\prime}}=j}$ are independent unless $v w$ and $v^{\prime} w^{\prime}$ share a vertex, in which case their covariance is at most one. It follows that $\operatorname{Var}\left(e\left(P_{i}^{\prime}, P_{j}^{\prime}\right)\right)$ is bounded by $2 \operatorname{hom}\left(P_{2}, G_{n}\right)$; the factor 2 arises since we may put the common vertex of two incident edges into $P_{i}$ or $P_{j}$. But $\operatorname{hom}\left(P_{2}, G_{n}\right) \leq 2 e\left(G_{n}\right) \Delta\left(G_{n}\right)$, which is $o\left(n^{4} p^{2}\right)$ by assumption. Hence, for any $\varepsilon$, the probability that

$$
\left|\frac{e\left(P_{i}^{\prime}, P_{j}^{\prime}\right)}{n^{2} p}-\frac{m_{i j}}{k^{2}}\right| \leq \varepsilon
$$

holds for $1 \leq i, j \leq k$ is at least $99 \%$, provided $n$ is large enough.

From the comments above, if $n$ is large enough, there is a partition $\Pi^{\prime}$ for which both (69) and (70) hold. Starting from such a partition and moving at most $O(\sqrt{n})=o(n)$ vertices of $L$ (the set of low-degree vertices) between parts, we may find a balanced partition with almost the same density matrix. In other words, we may find an element of $\mathcal{M}_{k}\left(G_{n}\right)$ close to $M$, completing the proof.

If $n p \rightarrow \infty$, then the condition of Lemma 6.12 that $\Delta\left(G_{n}\right)=o\left(n^{2} p\right)$ holds trivially, since $\Delta\left(G_{n}\right) \leq n=o\left(n^{2} p\right)$. When $n p$ is bounded, this condition is necessary. Taking $G_{n}$ to be a star, for example, every partition of $V\left(G_{n}\right)$ has the property that there is one part meeting all edges. But the corresponding kernel has partitions which are very far from having this property, in which, roughly speaking, the central vertex of the star has been split between parts.

\subsubsection{The partition metric in the denser setting.}

There are two reasons why we have waited until the present section on extremely sparse graphs before introducing the partition metric. One is that the natural motivation (distinguishing $G(n, 2 / n)$ from $G(n / 2, n / 2 ; 4 / n)$ ) arises in this setting. The second is that, from the point of view of distinguishing graphs, the partition metric adds nothing to $d_{\text {cut }}$ if $n p \rightarrow \infty$ : it is easy to see that $d_{\text {part }}$ and $d_{\text {cut }}$ are equivalent. (This is proved in the fully dense case in Borgs, Chayes, Lovász, Sós and Vesztergombi 18.) The key point is that one can identify the density matrix corresponding to a weakly $(\varepsilon, p)$-regular partition from the set of density matrices.

Lemma 6.13. Let $n p \rightarrow \infty$, and let $\left(G_{n}\right)$ be a sequence of graphs with $\left|G_{n}\right|=n$ satisfying the bounded density assumption, Assumption 4.1. Let $\kappa$ be a bounded kernel. Then $d_{\text {part }}\left(G_{n}, \kappa\right) \rightarrow 0$ if and only if $d_{\text {cut }}\left(G_{n}, \kappa\right) \rightarrow 0$. 
Proof. Suppose first that $d_{\text {cut }}\left(G_{n}, \kappa\right) \rightarrow 0$, i.e., that $d_{\text {cut }}\left(\kappa_{G_{n}}, \kappa\right) \rightarrow 0$. If $\kappa_{1}$ and $\kappa_{2}$ are any kernels with $d_{\text {cut }}\left(\kappa_{1}, \kappa_{2}\right)<d$, and $M \in \mathcal{M}_{k}\left(\kappa_{1}\right)$, then there is an $M^{\prime} \in \mathcal{M}_{k}\left(\kappa_{2}\right)$ whose entries differ from those of $M$ by at most $k^{2} d$ : one simply takes the corresponding partition for $\kappa_{2}$, after rearranging so that $\left\|\kappa_{1}-\kappa_{2}\right\|_{\text {cut }}<d$. It follows that $d_{\mathrm{H}}\left(\mathcal{M}_{k}\left(\kappa_{1}\right), \mathcal{M}_{k}\left(\kappa_{2}\right)\right) \leq k^{2} d_{\text {cut }}\left(\kappa_{1}, \kappa_{2}\right)$. Hence, $d_{\mathrm{H}}\left(\mathcal{M}_{k}\left(\kappa_{G_{n}}\right), \mathcal{M}_{k}(\kappa)\right) \rightarrow 0$. Using Lemma 6.12, it follows that $d_{\text {part }}\left(G_{n}, \kappa\right) \rightarrow 0$.

Now suppose that $d_{\text {part }}\left(G_{n}, \kappa\right) \rightarrow 0$ which, from Lemma 6.12 implies that $d_{\text {part }}\left(\kappa_{G_{n}}, \kappa\right) \rightarrow 0$. By the index $\operatorname{ind}(M)$ of a density matrix $M=\left(m_{i j}\right) \in$ $\mathcal{M}_{k}\left(\kappa_{G_{n}}\right)$ we mean simply $k^{-2} \sum m_{i j}^{2}$, which is also $\left\|\kappa_{G_{n}} / \Pi\right\|_{2}^{2}$ where $\Pi$ is a partition giving $M$ as density matrix, and $\kappa_{G_{n}} / \Pi$ is the corresponding averaged kernel. Let $f(k, \varepsilon) \geq k$ be a function to be specified later. A $k$-by- $k$ density matrix $M \in \mathcal{M}_{k}\left(\kappa^{\prime}\right)$ is locally $\varepsilon$-optimal for a kernel $\kappa^{\prime}$ if

$$
\sup _{\ell \leq f(k, \varepsilon)} \sup _{M^{\prime} \in M_{\ell}\left(\kappa^{\prime}\right)} \operatorname{ind}\left(M^{\prime}\right) \leq \operatorname{ind}(M)+\varepsilon,
$$

i.e., if $M$ has almost maximal index among density matrices with not too many parts. Fix $\varepsilon>0$. Since $G_{n}$ has bounded density, whenever $n$ is large enough as a function of $k$, any density matrix in $\mathcal{M}_{k}\left(\kappa_{G_{n}}\right)$ has index at most $C$. It follows that there is a $K=K(\varepsilon)$ such that, for $n$ large enough, every $G_{n}$ has some locally optimal density matrix $M_{k}(n)$ of size at most $K$. (This statement is a key part of the proof of Szemerédi's Lemma.)

Since $d_{\text {part }}\left(\kappa_{G_{n}}, \kappa\right) \rightarrow 0$, if $n$ is large enough, there is an $M_{k}^{\prime}(n) \in \mathcal{M}_{k}(\kappa)$ with all entries within $\varepsilon /(10 C)$ of those of $M_{k}(n)$. It follows that ind $\left(M_{k}^{\prime}(n)\right) \geq$ $\operatorname{ind}\left(M_{k}(n)\right)-\varepsilon / 2$. Similarly, for $n$ large, every $M \in \cup_{\ell \leq f(k, \varepsilon)} \mathcal{M}_{\ell}(\kappa)$ has all entries within $\varepsilon /(10 C)$ of some $M^{\prime} \in \cup_{\ell \leq f(k, \varepsilon)} \mathcal{M}_{\ell}(\kappa)$, which implies $\operatorname{ind}(M) \leq$ $\operatorname{ind}\left(M^{\prime}\right)+\varepsilon / 2$. It follows that $M_{k}^{\prime}(n)$ is locally $2 \varepsilon$-optimal for $\kappa$.

Recall that a partition $\Pi$ of $[0,1]$ is weakly $(\varepsilon, p)$-regular with respect to a kernel $\kappa^{\prime}$ if the corresponding averaged kernel $\kappa^{\prime} / \Pi$ satisfies $\left\|\kappa^{\prime} / \Pi-\kappa^{\prime}\right\|_{\text {cut }} \leq \varepsilon$. The proof of Lemma 4.3 (a sparse form of the Frieze-Kannan form of Szemerédi's Lemma) shows that if $G_{n}$ has bounded density, then there is a function $f(k, \varepsilon)$ such that, if $n \geq n_{0}(k, \varepsilon)$ and $M \in \mathcal{M}_{k}\left(\kappa_{G_{n}}\right)$ is locally $\varepsilon$-optimal, then the corresponding partition of $\kappa_{G_{n}}$ is weakly $(\varepsilon, p)$-regular; the same applies to $\kappa$. It follows that for $n$ large, identifying each density matrix with a corresponding kernel, we have $d_{\text {cut }}\left(\kappa_{G_{n}}, M_{k}(n)\right), d_{\text {cut }}\left(M_{k}(n), M_{k}^{\prime}(n)\right)$ and $d_{\text {cut }}\left(M_{k}^{\prime}(n), \kappa\right)$ all of order $O(\varepsilon)$. Since $\varepsilon$ was arbitrary, it follows that $d_{\text {cut }}\left(\kappa_{G_{n}}, \kappa\right) \rightarrow 0$, as required.

In the light of Corollary 4.7, Lemma 6.13 implies that $\left(G_{n}\right)$ is Cauchy with respect to $d_{\text {part }}$ if and only if it is Cauchy with respect to $d_{\text {cut }}$.

The bounded density assumption in Lemma 6.13, which is trivially satisfied in the fully dense case $p=\Theta(1)$, is necessary in general. This can be seen by considering, for example, a graph $G_{n}$ made up of $n / m$ complete graphs of order $m$, with $m \sim 2 p n=o(n)$ chosen so that $G_{n}$ has $p n^{2}$ edges. As noted above, by compactness, any sequence with $e\left(G_{n}\right)=O\left(p n^{2}\right)$ has a subsequence that is Cauchy with respect to $d_{\text {part }}$ (here, in fact, the original sequence is Cauchy). 
However, it is easy to check that no subsequence of $\left(G_{n}\right)$ is Cauchy with respect to $d_{\text {cut }}$.

The proof of Lemma 6.13 applies just as well to kernels as to graphs (and one can in any case approximate kernels by dense graphs), showing that $d_{\text {part }}\left(\kappa_{n}, \kappa\right) \rightarrow$ 0 if and only if $d_{\text {cut }}\left(\kappa_{n}, \kappa\right) \rightarrow 0$. It follows that $d_{\text {part }}$ induces a metric on $\mathcal{K}$, the set of kernels quotiented by equivalence, and that $d_{\text {part }}$ and $d_{\text {cut }}$ give rise to the same topology on $\mathcal{K}$. This was proved by Borgs, Chayes, Lovász, Sós and Vesztergombi [18] in their study of the dense case, as part of their Theorem 3.5.

\subsubsection{The partition metric and random graphs}

Let us return to our main focus in this section, the extremely sparse case $p=1 / n$. Our hope was that in this setting the partition metric might play the role of the cut metric in the denser setting, showing, for example, that a random sequence $\left(G_{1 / n}(n, \kappa)\right)$ has a limit with probability 1 , and that this limit is different for different $\kappa$.

For $d_{\text {cut }}$, in the denser setting, the limit was $\kappa$ itself, but here we cannot hope for this. Indeed, it is very easy to check that in $G(n, c / n)$, the largest and smallest balanced cuts have sizes that differ from $\mathrm{cn} / 4$ (the expected size of a random cut) by order $n$. Indeed, using the greedy algorithm to assign vertices one by one to a part of the bipartition, it is easy to construct such a cut. For the best current bounds on the largest cut in $G(n, c / n)$, see [5], 22] and [23] (for related results, see [7, 34, 37, [26], 24]). In statistical physics language, the problem is that when $p=1 / n$, the entropy and energy terms balance: a given cut is 'only' exponentially unlikely to have $1 \%$ fewer edges than the expected number (as opposed to superexponentially unlikely when $n p \rightarrow \infty$ ), but there are exponentially many cuts, so some atypical cuts will exist.

Despite the observation above, it is extremely likely that $\left(G_{1 / n}(n, \kappa)\right)$ is Cauchy with respect to $d_{\text {part }}$ with probability 1, i.e., that there is a limit point (depending only on $\kappa$ ) in the space $\prod_{k} \mathcal{C}\left(\mathcal{M}_{k}\right)$ defined above, even though this limit is not given by $\kappa$ in the way one might expect.

Conjecture 6.14. For any bounded kernel $\kappa$, the random sequence $G_{1 / n}(n, \kappa)$ is Cauchy with respect to $d_{\text {part }}$ with probability 1 .

Note that the analogue of Conjecture 6.14 with $n p \rightarrow \infty$ is trivial, since Chernoff's inequality shows that $G_{p}(n, \kappa)$ and $\kappa$ are close in the cut metric, and hence in $d_{\text {part }}$. While Conjecture 6.14 is very likely to be true, it may also be rather hard to prove, since it would imply the convergence of many quantities associated to $G(n, c / n)$ (such as the normalized size of the largest cut) that are not known to converge. For example, one can use partitions to pick out the size of the largest independent set within $o(n)$ (in this sparse setting, an almost independent set, spanning $o(n)$ edges, contains an independent set of almost the same size), so Conjecture 6.14 implies the following statement: there is a function $\beta(c)$ such that the independence number of $G(n, c / n)$ is $\beta(c) n+o_{\mathrm{p}}(n)$ as $n \rightarrow \infty$ with $c$ fixed; in particular, the independence number is concentrated for each $n$. While concentration is well known and easy to prove, at the moment it 
is not known that the independence number can't 'jump around' as $n$ increases, although of course this is extremely implausible. For a discussion of this, see, for example, Gamarnik, Nowicki and Swirszcz [28]; surprisingly, for $c \leq e$, convergence is known: it follows from results of Karp and Sipser 31.

The same concentration applies to $d_{\text {part }}$, as shown by the following result.

Theorem 6.15. Let $\kappa$ be a bounded kernel, let $k \geq 2$ be fixed, and let $G_{n}=$ $G_{1 / n}(n, \kappa)$. For each $n$ there is a set $Y_{n} \in \mathcal{M}_{k}$ such that $d_{\mathrm{H}}\left(\mathcal{M}_{k}\left(G_{n}\right), Y_{n}\right)$ converges to 0 in probability.

Proof. Since $\mathcal{M}_{k}$ is compact, from the definition of the Hausdorff metric it is enough to show that for any given point $M \in \mathcal{M}_{k}$ the random variable $Z_{n}=d_{\mathrm{H}}\left(\mathcal{M}_{k}\left(G_{n}\right),\{M\}\right)$ is concentrated around its mean as $n \rightarrow \infty$. Taking an $\varepsilon$-net in $\mathcal{M}_{k}$, the result then follows.

Roughly speaking, since the real-valued random variable $Z_{n}$ changes by order $1 / n$ if we add or delete an edge of $G_{n}$, concentration of $Z_{n}$ follows by standard martingale arguments. One must be a little careful, however, for two reasons. Firstly, we cannot afford to use the edge-exposure martingale, as that has too many steps. Using vertex exposure, one must consider the possibility of large degrees. Secondly, the 'type variables' $x_{1}, \ldots, x_{n}$ introduce some dependence between edges. There are many ways of working around these problems. One possibility is as follows.

Let $c=\sup \kappa<\infty$. We may couple $G_{n}$ and $G_{n}^{\prime}=G(n, c / n)$ in a natural way so that $G_{n} \subset G_{n}^{\prime}$. Indeed, first construct $G_{n}^{\prime}$, then choose the types $x_{1}, \ldots, x_{n}$, then keep each edge $i j$ of $G_{n}^{\prime}$ with probability $\kappa(i, j) / c$, independently of the others. It is easy to see that the set of edges remaining has the distribution of $G_{n}$. (This construction is also used by Bollobás, Janson and Riordan [11.)

For the moment, let us condition on $G_{n}^{\prime}$, fixing a possible graph $G_{n}^{\prime}$ with $e\left(G_{n}^{\prime}\right) \leq 10 \mathrm{cn}$ and $\Delta\left(G_{n}^{\prime}\right) \leq n^{1 / 10}$, say. We can construct $G_{n}$ from $G_{n}^{\prime}$ using a sequence of $n+2 e\left(G_{n}^{\prime}\right)$ independent uniform $U[0,1]$ random variables: one, $x_{i}$, for each vertex, and one, $w_{i j}$, for each edge $i j$ of $G_{n}^{\prime}$. Indeed, we simply keep the edge $i j$ of $G_{n}^{\prime}$ if and only if $w_{i j} \leq \kappa\left(x_{i}, x_{j}\right) / c$. Changing one of the $w_{i j}$ adds or deletes at most one edge of $G_{n}$, and so changes $Z_{n}$ by at most $k^{2} / n$. Changing one of the $x_{i}$ only affects the presence in $G_{n}$ of edges of $G_{n}^{\prime}$ incident with vertex $i$. By assumption, there are at most $n^{1 / 10}$ such edges, so changing $x_{i}$ changes $Z_{n}$ by at most $k^{2} n^{-9 / 10}$. Since we make only $O(n)$ choices, it follows using the Hoeffding-Azuma inequality that, given $G_{n}^{\prime}$ with the above properties, $Z_{n}$ is concentrated about its mean $\mathbb{E}\left(Z_{n} \mid G_{n}^{\prime}\right)$.

Since almost every $G_{n}^{\prime}=G(n, c / n)$ satisfies the bounds on $e\left(G_{n}^{\prime}\right)$ and $\Delta\left(G_{n}^{\prime}\right)$ above, it remains only to show that $\mathbb{E}\left(Z_{n} \mid G_{n}^{\prime}\right)$ is concentrated. For this it is more convenient to consider the graph $G_{n}^{\prime \prime}$ obtained as follows: choose $c n / 2$ edges independently and uniformly at random from all $\left(\begin{array}{l}n \\ 2\end{array}\right)$ possible edges, deleting any repeated edges. It is easy to see that $G_{n}^{\prime \prime}$ and $G(n, c / n)$ may be coupled to agree within $o_{\mathrm{p}}(n)$ edges. Since adding or deleting an edge of $G$ changes $\mathbb{E}\left(Z_{n} \mid G_{n}^{\prime}=G\right)$ by at most $k^{2} / n$, it suffices to prove concentration of $\mathbb{E}\left(Z_{n} \mid G_{n}^{\prime}\right)$ when $G_{n}^{\prime}$ has the distribution $G_{n}^{\prime \prime}$. But this is immediate from the 
Hoeffding-Azuma inequality, since $G_{n}^{\prime \prime}$ is constructed from $O(n)$ independent choices each of which changes this expectation by at most $k^{2} / n$.

The result above shows that the random sets $\mathcal{M}_{k}\left(G_{n}\right)$ become concentrated as $n \rightarrow \infty$. The problem is that the points they become concentrated around might in principle jump around as $n$ varies.

Even without a proof of Conjecture 6.14 it still makes good sense to ask whether $d_{\text {part }}$ at least separates different random graph models $G_{1 / n}(n, \kappa)$. As before, let us write $d_{\text {edit }}\left(G_{1}, G_{2}\right)$ for the normalized edit distance between two graphs $G_{1}, G_{2}$ with $\left|G_{1}\right|=\left|G_{2}\right|=n$, i.e., for $1 /\left(n^{2} p\right)$ times the minimal number of edge changes (additions or deletions) that must be made to $G_{1}$ to produce a graph isomorphic to $G_{2}$. By a random graph model we simply mean a sequence of probability measures on the sets of $n$-vertex graphs. We say that two random graph models are essentially equivalent if one can couple the corresponding $n$ vertex random graphs $G_{n}$ and $G_{n}^{\prime}$ so that $\mathbb{E}\left(d_{\text {edit }}\left(G_{n}, G_{n}^{\prime}\right)\right)=o(1)$.

Conjecture 6.16. Let $\kappa_{1}$ and $\kappa_{2}$ be bounded kernels such that the models $G_{1 / n}\left(n, \kappa_{1}\right)$ and $G_{1 / n}\left(n, \kappa_{2}\right)$ are not essentially equivalent. Then the expected $d_{\text {part }}$ distance between $G_{1 / n}\left(n, \kappa_{1}\right)$ and $G_{1 / n}\left(n, \kappa_{2}\right)$ is bounded below as $n \rightarrow \infty$.

Note that the distance between the $n$ vertex graphs is concentrated by Theorem 6.15. Of course, any proof of Conjecture 6.16 is likely to involve understanding for which pairs of kernels the corresponding models $G_{1 / n}(n, \kappa)$ are essentially equivalent. We discuss this briefly in the next subsection.

\subsection{Which kernels give the same random graphs?}

We have already seen a rather simple example of two kernels that are not equivalent (in the sense of Subsection 2.4), which nonetheless give rise to essentially equivalent sparse random graphs: we may take any two non-equivalent kernels $\kappa_{1}, \kappa_{2}$ corresponding to the same subcritical branching process. Of course, the corresponding random graphs have a rather simple structure, since they are made up of (essentially) only small tree components. Unfortunately, (or interestingly, depending on ones point of view) a simple modification of this example gives examples with more complex structure.

Example 6.17. Let $c>1$ be constant, and let $\kappa_{1}$ and $\kappa_{2}$ be bounded kernels corresponding to the same branching process. Writing $c+\kappa_{i}$ for the pointwise sum of the constant kernel $c$ and the kernel $\kappa_{i}$, the models $G_{1 / n}\left(n, c+\kappa_{i}\right)$, $i=1,2$, are essentially equivalent. To see this, we realize $G_{1 / n}\left(n, c+\kappa_{i}\right)$ by first constructing $G_{1 / n}\left(n, \kappa_{i}\right)$, and then adding each non-edge with probability $c / n$, and then adding each non-edge with a tiny probability to get the edge probabilities exactly right. We can ignore the last step since it adds $O_{\mathrm{p}}(1)=$ $o_{\mathrm{p}}(n)$ edges. Constructing the graphs $G_{1 / n}\left(n, \kappa_{i}\right)$ first, we may relabel the vertices of these graphs so that they coincide apart from $o_{\mathrm{p}}(n)$ edges. We may then add each possible edge to both graphs simultaneously with probability $c / n$ to obtain (essentially) the required coupling of the graphs $G_{1 / n}\left(n, c+\kappa_{i}\right)$. 
In general, we believe the following is an interesting question.

Question 6.18. For which pairs of supercritical kernels $\kappa_{1}, \kappa_{2}$ are the models $G_{1 / n}\left(n, \kappa_{1}\right)$ and $G_{1 / n}\left(n, \kappa_{2}\right)$ essentially equivalent?

Certainly, any such pair must satisfy $\pi\left(\kappa_{1}\right)=\pi\left(\kappa_{2}\right)$, otherwise the models are distinguished by their tree counts. A simple answer to Question 6.18 would be important for the general understanding of the sparse inhomogeneous model of Bollobás, Janson and Riordan [10.

Since Question 6.18 is rather open ended, let us focus on one particular example: the pair consisting of the constant kernel $c$ and the kernel $\kappa_{c+\delta, c-\delta}$ defined in (67), with $0<|\delta|<c$. The cases $\delta$ positive and $\delta$ negative may behave differently, although we do not expect this to be the case. For $0<\delta^{\prime}<\delta$, or $0>$ $\delta^{\prime}>\delta$, one can construct $G_{1 / n}\left(n, \kappa_{c+\delta^{\prime}, c-\delta^{\prime}}\right)$ from $G_{1 / n}\left(n, \kappa_{c+\delta, c-\delta}\right)$ by deleting each edge independently with a certain probability, and then adding in each non-edge with an appropriate probability. It follows that if $G_{1 / n}\left(n, \kappa_{c+\delta, c-\delta}\right)$ and $G(n, c / n)$ are essentially equivalent, then so are $G_{1 / n}\left(n, \kappa_{c+\delta^{\prime}, c-\delta^{\prime}}\right)$ and $G(n, c / n)$. Hence there is an interval $I(c)$ such that $G_{1 / n}\left(n, \kappa_{c+\delta, c-\delta}\right)$ and $G(n, c / n)$ are essentially equivalent for all $\delta \in I(c)$, but for no $\delta \in[-c, c] \backslash I(c)$.

The construction in Example 6.17 shows that $[-1,1] \subset I(c)$ whenever $c>1$. On the other hand, it is not hard to show that for $c$ large, the most extreme balanced cuts in $G(n, c / n)$ contain $c n / 4 \pm \Theta(\sqrt{c} n)$ edges; for the best known bound on the largest cut see [5], 22] and [23]. Since a typical $G_{1 / n}\left(n, \kappa_{c+\delta, c-\delta}\right)$ clearly contains a balanced cut with $(c-\delta) n / 4+o_{\mathrm{p}}(n)$ edges, it follows that $I(c) \subset[-A \sqrt{c}, A \sqrt{c}]$ for $c$ large, where $A$ is an absolute constant. It is not hard to convince oneself that the second bound is closer to the truth, although we do not have a proof. In fact, we believe that the endpoints of $I(c)$ are exactly $\pm \sqrt{c}$ for every $c \geq 1$, although we make no guess as to whether these endpoints are included.

Conjecture 6.19. Let $c>1$ and $-c \leq \delta \leq c$ be constants. If $\delta<\sqrt{c}$, then the models $G_{1 / n}\left(n, \kappa_{c+\delta, c-\delta}\right)$ and $G(n, c / n)$ are essentially equivalent. If $\delta>\sqrt{c}$, then they are not.

The model $G_{1 / n}\left(n, \kappa_{c+\delta, c-\delta}\right)$ is a special case of the planted bisection model $G\left(n ; p, p^{\prime}\right)$ : for any $p=p(n)$ and $p^{\prime}=p(n)$, the graph $G\left(n ; p, p^{\prime}\right)$ is constructed by partitioning its vertex set $[n]$ at random into two (almost) equal parts, and then joining any two vertices in the same part with probability $p$, and two vertices in different parts with probability $p^{\prime}$. The question of reconstructing the vertex partition given only the graph has received considerable attention, generally with emphasis on polynomial-time algorithms for $p, p^{\prime}$ satisfying suitable conditions; see, for example, Boppana [13, and, for a linear expected time algorithm, Bollobás and Scott [12. Most such results are for graphs with average degree tending to infinity, but Coja-Oghlan 21 proved results that include the extremely sparse case, showing that one can find a minimum balanced cut in $G_{1 / n}\left(n, \kappa_{c+\delta, c-\delta}\right)$ in polynomial time whenever $\delta>\Theta(1+c \log (c))$. The connection with Conjecture 6.19 is rather loose, but nonetheless interesting. 
Let us present another question that does seem to be closely related to Conjecture 6.19.

Question 6.20. When does the branching process $\mathfrak{X}_{\kappa_{c+\delta, c-\delta}}$ forget the type of the root?

To spell out what we mean by Question 6.20 let $T_{t}$ be the random labelled tree corresponding to the first $t$ generations of $\mathfrak{X}=\mathfrak{X}_{\kappa_{c+\delta, c-\delta}}$, with each vertex labelled by the type of the corresponding particle. Here we take the types to be 1 and 2 , corresponding to $x \leq 1 / 2$ and $x>1 / 2$. Note that each particle has $\operatorname{Po}((c+\delta) / 2)$ children of its own type, and $\operatorname{Po}((c-\delta) / 2)$ of the opposite type. Recall that, since $\pi(\mathfrak{X})=\pi\left(\mathfrak{X}_{c}\right)$, the distribution of $T_{t}$ without labels is simply that of the first $t$ generations of a single-type branching process. Let $U_{t}$ be the tree obtained from $T_{t}$ by forgetting the labels of all vertices other than those at distance $t$ from the root, and let $p_{t}$ be the probability, conditional on $U_{t}$, that the root has type 1 , so $p_{t}$ is a random variable depending on $\mathfrak{X}$ via $U_{t}$. We say that $\mathfrak{X}$ forgets the type of the root if $p_{t} \stackrel{\mathrm{p}}{\rightarrow} 1 / 2$ as $t \rightarrow \infty$. It is easy to couple the branching processes $\mathfrak{X}(1)$ and $\mathfrak{X}(2)$ started with particles of type 1 and 2 , respectively, so that the tree structures always agree, and the expected number of label mismatches in level $t$ is $\delta^{t}$. It follows that $\mathfrak{X}$ forgets the type of the root if $\delta \leq 1$. Although we have not checked the details, we believe that $\mathfrak{X}$ forgets the type of the root if and only if $\delta \leq \sqrt{c}$; this is based on linearizing the natural recurrence describing the distribution of $p_{t+1}$ in terms of that of $p_{t}$. (Actually, one works with the distribution of $p_{t}$ given that the root has type 1.) This argument certainly shows that $\mathfrak{X}$ cannot forget the type of the root if $\delta>\sqrt{c}$; the reverse implication is not so clear.

Although we certainly have no proof, it seems likely that if $\mathfrak{X}=\mathfrak{X}_{\kappa_{c+\delta, c-\delta}}$ forgets the type of the root, then the models $G_{1 / n}\left(n, \kappa_{c+\delta, c-\delta}\right)$ and $G(n, c / n)$ are essentially equivalent. Roughly speaking, suppose that, given the global structure of $G_{1 / n}\left(n, \kappa_{c+\delta, c-\delta}\right)$, seen without types, we can somehow form a good guess as to which vertices at graph distance 100 from a given vertex $v$ are of type 1 and which of type 2. Even then, $v$ itself is (almost) equally likely to be of either type. This strongly suggests that one can get essentially no information about the vertex types from the graph, and hence that the types do not matter to the graph. This vague heuristic is very far from a proof, however!

In summary, it seems very likely that the answers to Conjecture 6.19 and Question 6.20 are closely related. In turn they may well be related to the question of when the maximum/minimum balanced cut distinguishes $G_{1 / n}\left(n, \kappa_{c+\delta, c-\delta}\right)$ from $G(n, c / n)$. We do not even have a guess as to the form of a more general answer to Question 6.18.

\subsection{General extremely sparse graphs}

So far in this section, we have considered only locally acyclic graphs. This was natural both when considering the metric $d_{\text {sub }}$, for which only counts of trees made sense when using the normalization used in the rest of the paper, and when considering the random graph model $G_{1 / n}(n, \kappa)$. There are many natural 
sequences of graphs with $\Theta(n)$ edges that are not treelike, however. A simple example is given by taking $G_{n}$ to consist of $n / 3$ vertex disjoint triangles, for $n$ a multiple of three. Clearly, this graph is not close to any locally treelike graph. It would be nice to have a notion of when two general graphs with $\Theta(n)$ edges are close, as well as a more general random model generating such graphs.

What distinguishes the union of $n / 3$ triangles from a Hamilton cycle $C_{n}$, say? The simplest answer is the number of triangles. Throughout this section we consider sequences $\left(G_{n}\right)$ with exponentially bounded tree counts, i.e., we assume that there is a constant $C$ such that $\limsup _{n \rightarrow \infty} t\left(T, G_{n}\right) \leq C^{e(T)}$ for every tree $T$. This condition is certainly satisfied if the graphs $G_{n}$ have bounded maximum degree, for example, and the reader may wish to think of this case for simplicity. In fact, as in Subsection 6.2 something weaker than exponential boundedness probably suffices, but exponential boundedness is a natural assumption. If $\left(G_{n}\right)$ has exponentially bounded (or indeed simply bounded) tree counts, then the number of embeddings or homomorphisms from any fixed graph $F$ into $G_{n}$ is $O(n)$.

For each fixed $F$, let

$$
\tilde{s}\left(F, G_{n}\right)=\operatorname{emb}\left(F, G_{n}\right) / n,
$$

and let $\tilde{t}\left(F, G_{n}\right)=\operatorname{hom}\left(F, G_{n}\right) / n$. As in previous sections, we can use the (now differently normalized) subgraph counts $\tilde{s}$ or $\tilde{t}$ to define a metric $\tilde{d}_{\text {sub }}$, by first mapping $G_{n}$ to

$$
\tilde{s}\left(G_{n}\right)=\left(\tilde{s}\left(F, G_{n}\right)\right)_{F \in \mathcal{F}} \in[0, \infty)^{\mathcal{F}} .
$$

As noted above, each coordinate is bounded for the sequences we consider, so $\tilde{s}$ maps into a compact subset $X$ of $[0, \infty)^{\mathcal{F}}$. Using any metric $d$ on $X$ giving rise to the product topology, we may then define $\tilde{d}_{\text {sub }}\left(G, G^{\prime}\right)=d\left(\tilde{s}(G), \tilde{s}\left(G^{\prime}\right)\right)$.

As in Subsection 6.2. one can view the (limiting) counts $\tilde{s}\left(F, G_{n}\right)$ as giving the moments of a certain distribution that we could instead study directly. Let $\mathcal{G}_{t}^{\mathrm{r}}$ be the set of isomorphism classes of connected finite rooted graphs with radius at most $t$, i.e., with every vertex at graph distance at most $t$ from the root. Also, let $\mathcal{G}^{\text {r }}$ be the set of all locally finite rooted (finite or infinite) graphs. As in Subsection 6.2 for $F \in \mathcal{G}_{t}^{\mathrm{r}}$ let

$$
p\left(F, G_{n}\right)=p_{t}\left(F, G_{n}\right)=\mathbb{P}\left(\Gamma_{\leq t}(v) \cong F\right),
$$

where $v$ is a vertex of $G_{n}$ chosen uniformly at random, and $\Gamma_{\leq t}(v)$ is the subgraph of $G_{n}$ induced by the vertices within distance $t$ of $v$, viewed as a rooted graph with root $v$. Since each $p_{t}\left(F, G_{n}\right)$ lies in $[0,1]$, trivially, any sequence $\left(G_{n}\right)$ has a subsequence along which $p_{t}\left(F, G_{n}\right)$ converges to some $p_{t}(F)$ for every $t$ and every $F \in \mathcal{G}_{t}^{\mathrm{r}}$. Furthermore, as in Subsection 6.2. if $\left(G_{n}\right)$ has bounded tree counts then it is easy to check that $\sum_{F \in \mathcal{G}_{t}^{r}} p_{t}(F)=1$, so $p_{t}$ is a probability distribution on $\mathcal{G}_{t}^{\mathrm{r}}$. Furthermore, these probability distributions for different $t$ are consistent in the natural sense, and so may be combined to form a probability distribution on $\mathcal{G}^{\mathrm{r}}$. For this reason, we say that a probability distribution $\pi$ on $\mathcal{G}^{\mathrm{r}}$ is the local limit of the sequence $\left(G_{n}\right)$ if

$$
p_{t}\left(F, G_{n}\right) \rightarrow \pi\left(\left\{G:\left.G\right|_{t}=F\right\}\right)
$$


for every $t \geq 1$ and every $F \in \mathcal{G}_{t}^{\mathrm{r}}$, where for $G \in \mathcal{G}^{\mathrm{r}}$, the graph $\left.G\right|_{t}$ is the subgraph of $G$ induced by the vertices within distance $t$ of the root.

Of course one can define a corresponding metric $d_{\text {loc }}$, by mapping each graph to the point $\left(p_{t}\left(F, G_{n}\right)\right) \in X=\prod_{t}[0,1]^{\mathcal{G}_{t}^{\mathrm{r}}}$, and then applying any metric on $X$ giving rise to the product topology. Just as for tree counts, under suitable assumptions, the local limit $\pi$ determines the limiting subgraph counts $\tilde{s}$ and vice versa. (One 'suitable assumption' is bounded maximum degree. In fact, as in Subsection 6.2 exponentially bounded tree counts will almost certainly do.) Thus the metrics $\tilde{d}_{\text {sub }}$ and $d_{\text {loc }}$ are equivalent. As before, it seems more natural to study the distribution of the neighbourhoods of vertices directly, rather than the subgraph counts, which are essentially moments of this distribution.

This notion of local limit is extremely natural. In fact, Benjamini and Schramm 4 used the same notion (but for a random rather than deterministic sequence $G_{n}$ ) to define a 'distributional limit' of certain random planar graphs; they showed that the random walk on the limiting graph is recurrent with probability 1 . The same notion in slightly different generality was studied by Aldous and Steele [2], under the name 'local weak limit', and Aldous and Lyons [1, where the term 'random weak limit' is used. Returning to basic questions about this notion of limit, perhaps the first is: which probability distributions on $\mathcal{G}^{\mathrm{r}}$ can arise in this way? As in Subsection 6.2, an easy necessary condition is that the distribution be shift invariant; we shall not repeat the definition, which is the same as in the tree case. The following question is due to Aldous and Lyons [1].

Question 6.21. Does every shift-invariant probability distribution on $\mathcal{G}_{\infty}^{\mathrm{r}}$ arise as the local limit of some sequence $\left(G_{n}\right)$ of graphs with $\left|G_{n}\right|=n$ ?

Just as in the tree case, it may make sense to restrict to graphs with bounded maximum degree, asking the analogue of Question 6.7

Benjamini and Schramm [4] noted the necessity of a condition equivalent to shift-invariance, which they called an 'intrinsic mass transport principle'. Aldous and Steele [2] also noted the necessity of shift-invariance, which they call 'involution invariance', and formulate slightly differently. Aldous and Lyons [1] defined a condition they called 'unimodularity,' which they showed to be equivalent to involution invariance, and stated Question 6.21 (formulated somewhat differently) as an especially important open question. (Lyons 38 referred to a proof of a positive answer to Question 6.21 but in a note added in proof said that this proof was incorrect.) Note that it does not matter here whether we consider a sequence of deterministic finite graphs, or a sequence of distributions on $n$-vertex graphs: for the purposes of Question 6.21 a distribution on connected $n$-vertex graphs may be well approximated by a much larger finite graph whose components have approximately the right distribution.

In one way, the metric $d_{\text {loc }}$ associated to the notion of local convergence seems to be the most natural measure of 'similarity' between general sparse graphs. However, while this notion captures local information well, it still loses global information: if $\left(G_{n}\right)$ has a certain local limit $\pi$, then the graphs $H_{2 n}$ formed by taking the disjoint union of two copies of $G_{n}$ have the same limit. 
(Indeed, $p_{t}\left(F, G_{n}\right)=p_{t}\left(F, H_{2 n}\right)$ for every connected graph $F$.) This shows that $d_{\text {loc }}$ fails to capture the global structure of the graph, and suggests that it makes sense to consider $d_{\text {loc }}$ and $d_{\text {part }}$ together; we shall do so in the next section.

\section{Further metrics, models and questions}

For fully dense graphs, with $\Theta\left(n^{2}\right)$ edges, the results of Borgs, Chayes, Lovász, Sós and Vesztergombi [17, 18, show that one single metric, say $d_{\text {cut }}$, effectively captures several natural notions of local and global similarity. Indeed, convergence in $d_{\text {cut }}$ is equivalent to convergence in the partition metric $d_{\text {part }}$ (a natural global notion) and to convergence in $\tilde{d}_{\text {sub }}$, i.e., convergence of all small subgraph counts, a natural local notion.

In the extremely sparse case, we have considered two metrics, the partition metric $d_{\text {part }}$ and the local metric $d_{\text {loc }}$, which respectively capture global and local similarity. Of course, one would like a single metric capturing both notions, and also the interaction between local and global properties. Fortunately, there is a natural combination of $d_{\text {part }}$ and $d_{\text {loc }}$.

\subsection{The coloured neighbourhood metric for extremely sparse graphs}

Let $G_{n}$ be a graph with $n$ vertices, and $k \geq 1$ an integer. We shall think of $G_{n}$ as having $\Theta(n)$ edges, though this is only relevant when we come to sequences $\left(G_{n}\right)$. Let $\Pi=\left(P_{1}, \ldots, P_{k}\right)$ be a partition of the vertex set of $G_{n}$, which we may think of as a (not necessarily proper) $k$-colouring of $G_{n}$. This time, for variety, we do not insist that the parts have almost equal sizes; this makes essentially on difference. Let $\mathcal{G}_{k, t}^{\mathrm{r}}$ be the set of isomorphism classes of $k$-coloured connected rooted graphs with radius at most $t$. For each $F \in \mathcal{G}_{k, t}^{\mathrm{r}}$, let $p_{k, t}\left(G_{n}, \Pi\right)(F)$ be the probability that the $t$-neighbourhood of a random vertex of the coloured graph $\left(G_{n}, \Pi\right)$ is isomorphic to $F$ as a coloured rooted graph, so $p_{k, t}\left(G_{n}, \Pi\right)$ is a probability distribution on $\mathcal{G}_{k, t}^{\mathrm{r}}$. Finally, let

$$
\mathcal{M}_{k, t}\left(G_{n}\right)=\left\{p_{k, t}\left(G_{n}, \Pi\right)\right\},
$$

where $\Pi$ runs over all $k$-partitions of $V\left(G_{n}\right)$. Thus $\mathcal{M}_{k, t}\left(G_{n}\right)$ is a finite subset of the space $\mathcal{P}\left(\mathcal{G}_{k, t}^{\mathrm{r}}\right)$ of probability distributions on $\mathcal{G}_{k, t}^{\mathrm{r}}$. Of course, one can view $\mathcal{M}_{k, t}\left(G_{n}\right)$ as a multiset, in which case it has exactly $k^{n}$ elements, one for each colouring. However, as for the partition metric in Subsection 6.4 it turns out to be better to ignore the multiplicities.

The space $\mathcal{P}\left(\mathcal{G}_{k, t}^{\mathrm{r}}\right)$ of probability distributions on $\mathcal{G}_{k, t}^{\mathrm{r}}$ is naturally viewed as a metric space, with the total variation distance $d_{\mathrm{TV}}$ between two distributions as the metric. In other words, regarding $\mathcal{P}\left(\mathcal{G}_{k, t}^{\mathrm{r}}\right)$ as a subset of the unit ball of $\ell_{1}$

in $\mathbb{R}^{\mathcal{G}_{k, t}^{\mathrm{r}}}$, we simply take the $\ell_{1}$-metric on this set. Let $d_{\mathrm{H}}$ denote the Hausdorff distance between compact subsets of $\mathcal{P}\left(\mathcal{G}_{k, t}^{\mathrm{r}}\right)$, defined with respect to $d_{\mathrm{TV}}$. Then 
we may define the coloured neighbourhood metric $d_{\mathrm{cn}}$ by

$$
d_{\mathrm{cn}}\left(G, G^{\prime}\right)=\sum_{k \geq 1} \sum_{t \geq 1} 2^{-k-t} d_{\mathrm{H}}\left(\mathcal{M}_{k, t}(G), \mathcal{M}_{k, t}\left(G^{\prime}\right)\right),
$$

say. (As before, we can instead use any metric on $\prod_{t, k} \mathcal{P}\left(\mathcal{G}_{k, t}^{\mathrm{r}}\right)$ giving rise to the product topology.) If we restrict our attention to graphs with maximum degree at most some constant $\Delta$, then the corresponding sets $\mathcal{G}_{k, t}^{\mathrm{r}}$ are finite, so each $\mathcal{P}\left(\mathcal{G}_{k, t}^{\mathrm{r}}\right)$ is compact, and any sequence $\left(G_{n}\right)$ has a subsequence that is Cauchy with respect to $d_{\mathrm{cn}}$, and in fact converges to a limit point consisting of one compact subset of $\mathcal{P}\left(\mathcal{G}_{k, t}^{\mathrm{r}}\right)$ for each $k, t$. In fact, it is not hard to check that whenever $\left(G_{n}\right)$ has bounded tree counts (i.e., contains $O(n)$ copies of any fixed tree $T$ ), it has a convergent subsequence with respect to $d_{\mathrm{cn}}$. Of course, as before, we can combine the limiting subsets of $\mathcal{P}\left(\mathcal{G}_{k, t}^{\mathrm{r}}\right)$ as $t$ varies. Also, as in Subsection 6.4 there may be circumstances in which it is better to view $\mathcal{M}_{k, t}\left(G_{n}\right)$ as a multiset after all, and use the matching distance between such multisets to define a coloured neighbourhood matching metric $d_{\mathrm{cn}-\mathrm{m}}$.

Taking just $k=1$ above, we recover the notion of local limit. On the other hand, the set $\mathcal{M}_{k}$ used to define the partition metric can be recovered from $\mathcal{M}_{k, 1}$. (The latter set codes, for each partition, how many vertices there are in each part, and how many neighbours in each part each vertex has. From this information one can calculate the number of edges between each pair of parts.)

\subsection{Models for metrics}

In the fully dense case, there is a very natural correspondence between limit points of sequences converging in $d_{\text {cut }}$, and the inhomogeneous random graph model $G(n, \kappa)$. In general, given any metric, we can ask whether there is a corresponding random graph model: for each metric $d$ on some class of (sparse) graphs satisfying certain restrictions, we can ask the following question.

Question 7.1. Given a metric $d$, can we find a 'natural' family of random graph models with the following two properties: (i) for each model, the sequence of random graphs $\left(G_{n}\right)$ generated by the model is Cauchy with respect to $d$ with probability 1 , and (ii) for any sequence $\left(G_{n}\right)$ with $\left|G_{n}\right|=n$ that is Cauchy with respect to $d$, there is a model from the family such that, if we interleave $\left(G_{n}\right)$ with a sequence of random graphs from the model, the resulting sequence is still Cauchy with probability 1.

Although this question is rather vague, for $d=d_{\text {cut }}$ the answer is yes in the dense case, since $\left(G_{n}\right)$ is Cauchy if and only if $d_{\text {cut }}\left(G_{n}, \kappa\right) \rightarrow 0$ for some kernel $\kappa$, while the dense inhomogeneous random graphs $G(n, \kappa)$ converge to $\kappa$ in $d_{\text {cut }}$ with probability 1 . Thus our family consists of one model $G(n, \kappa)$ for each kernel $\kappa$ (to be precise, for each equivalence class of kernels under the relation $\sim$ defined in Subsection 2.4 .

In the sparse case we do not have an entirely satisfactory answer for any of the metrics considered in this paper. Assuming that $n p \rightarrow \infty$, there is an almost 
completely satisfactory answer for $d_{\text {cut }}$ : if we impose the 'bounded density' assumption, Assumption 4.1, then Corollary 4.7 and Lemma 4.10 show that the sparse inhomogeneous models $G_{p}(n, \kappa)$ answer Question 7.1 . For $d_{\text {sub }}$, defined with respect to certain restricted sets of subgraphs, the results in Section 5 (in particular, Theorem 5.17) show that once again $G_{p}(n, \kappa)$ answers this question for suitably restricted sequences.

In the extremely sparse case, $G_{1 / n}(n, \kappa)$ is very unsatisfactory as a model for an arbitrary sequence of sparse graphs, since it produces graphs with essentially no cycles. The following natural model proposed by Bollobás, Janson and Riordan [1] is rather more general. In the uniform case, generalizing $G(n, c / n)$, assign a weight $w_{F}$ to each fixed graph $F$. To generate a random graph with $n$ vertices, starting from the empty graph, for each $F$ add each of the $\Theta\left(n^{|F|}\right)$ possible copies of $F$ with probability $w_{F} / n^{|F|-1}$, deleting any duplicate edges. Note that, on average, we add $\Theta(n)$ copies of each graph $F$. The point is that this model produces graphs with $\Theta(n)$ edges, but (in general) $\Theta(n)$ triangles, and indeed $\Theta(n)$ copies of any fixed graph $F$.

In the general case, Bollobás, Janson and Riordan [11] start from a kernel family $(\kappa)$ consisting of one kernel $\kappa_{F}$ for each connected finite graph $F$; the kernel $\kappa_{F}$ is simply a measurable function on $[0,1]^{V(F)}$ that is symmetric under the action of the automorphism group of $F$. To construct the random graph $G(n,(\kappa))$, choose $x_{1}, \ldots, x_{n}$ independently and uniformly from $[0,1]$, and then for each $F$ and each set $v_{1}, \ldots, v_{k}$ of $k=|F|$ vertices, insert a copy of $F$ with vertex set $v_{1}, \ldots, v_{k}$ with probability $\kappa_{F}\left(x_{v_{1}}, \ldots, x_{v_{k}}\right) / n^{k-1}$. For full details, see 11 .

While the model $G(n,(\kappa))$ is much more general than $G_{1 / n}(n, \kappa)$, it still has its limitations. It is not hard to see that the asymptotic degree distribution of $G(n,(\kappa))$ will be a mixture of compound Poisson distributions (rather than the mixture of Poisson distributions one obtains for $\left.G_{1 / n}(n, \kappa)\right)$. In particular, there is no kernel family for which $G(n,(\kappa))$ produces graphs in which (almost) all vertices have degree 3, say. A positive answer to Question 7.1 for either of the metrics $d_{\text {loc }}$ or $d_{\mathrm{cn}}$ discussed in Subsections 6.6 and 7.1 would involve, among other things, models that produce graphs with arbitrary given degree distributions (with finite expectation, say, or perhaps bounded, to keep things simple). Of course this is easy, but such an answer would require much more - it would require producing all possible distributions of local structure. Even for $d_{\text {loc }}$, this is likely to be hard, since one would presumably have to first understand the possible limiting distributions, which would involve answering Question 6.21. For $d_{\mathrm{cn}}$, much more is needed: one needs to understand the possible combinations of local and global structure.

Let us give one very simple example of the kind of model we have in mind, associated to $d_{\text {loc }}$ in the extremely sparse case. Let parameters $n$ and $d$ be given; we shall think of $d$ fixed as $n \rightarrow \infty$. Assuming that $n d$ is a multiple of three, let $T(n, d)$ be the random graph obtained as follows: start with $n$ vertices, each of which has $d$ 'stubs' associated to it. Take a uniformly random partition of the set of $n d$ stubs into $n d / 3$ triplets, and add a triangle corresponding to each triplet, sitting on the vertices that the stubs in the triplet are associated to. In 
general, $T(n, d)$ is a multigraph, but it will be very close to a simple graph (in fact, $T(n, d)$ will be simple with probability bounded away from 0 ). The model $T(n, d)$ is a natural 'triangle version' of the random regular graph, generated via the configuration model.

It is not hard to see that in $T(n, d)$ every (or, if we make the graph simple, almost every) vertex has degree $2 d$, and is in exactly $d$ (edge-disjoint) triangles. Furthermore, other than this, $T(n, d)$ has no local structure: the local limit of the sequence $T(n, d)$ is an infinite tree of triangles. Thus $T(n, d)$ is an appropriate model for certain Cauchy sequences in $d_{\text {loc }}$. Of course one can construct many other models along these lines, but it is hard to imagine that all Cauchy sequences can be covered in this way!

As we have seen, in the extremely sparse case, Question 7.1 is likely to be very hard to answer for the metrics we have considered. Nonetheless, it may be possible to answer the same question for weaker metrics, or to provide partial answers. Such partial answers would hopefully provide great insight into the structure of the set of sparse graphs.

\subsection{Subgraph counts revisited}

The models discussed in the previous section suggest both new models and new metrics for denser graphs. One example is the triangle configuration model $T(n, d)$ for any function $d=d(n)$. More simply, for any function $p=p_{K_{3}}(n)$ we could consider the random graph on $n$ vertices obtained by including each of the $\left(\begin{array}{l}n \\ 3\end{array}\right)$ possible triangles with probability $p$, independently of the others. More generally, there are natural denser versions of the model of Bollobás, Janson and Riordan [11]: given a kernel family $(\kappa)$ and, for each fixed graph $F$, a normalizing function $p_{F}(n)$, we choose types $x_{1}, \ldots, x_{n}$ independently and uniformly at random and then, for each $F$, add each possible copy of $F$ with vertex set $v_{1}, \ldots, v_{k}$ with probability $\kappa_{F}\left(x_{v_{1}}, \ldots, x_{v_{k}}\right) p_{F}(n)$.

In the sparse case, there was one natural normalization: at least up to a constant factor we should take $p_{F}=n^{1-|F|}$, so we add $\Theta(n)$ copies of each graph $F$. In the denser case, a huge family of normalizations are possible: we can take each $p_{F}$ to be any function of $n$ bounded by 1 . Of course, certain restrictions will be necessary for the model to make much sense; otherwise, for example, the copies of some $F_{1}$ added directly may be swamped by copies of $F_{1}$ arising as subgraphs of some $F_{2}$, in which case there was no point adding any copies of $F_{1}$ directly. However, many different normalizations will certainly be interesting: for example, we can produce graphs with, say, $\Theta\left(n^{4 / 3}\right)$ edges and $\Theta\left(n^{a}\right)$ triangles, for any $0<a \leq 4 / 3$. Indeed, to do so we need only two kernels, one for edges (which we may take bipartite if needed), and one for triangles.

If, for some reason, we are considering graphs with, say, around $n^{4 / 3}$ and $n^{6 / 5}$ triangles, which is many more triangles than expected in $G\left(n, n^{-2 / 3}\right)$, then the triangles are an important part of the structure, so in comparing two such graphs we should certainly compare the number of triangles, normalized by dividing by $n^{6 / 5}$. This suggests a family of metrics generalizing $d_{\text {sub }}$ and $\tilde{d}_{\text {sub }}$.

For each $F \in \mathcal{F}$ let $N_{F}=N_{F}(n)$ be a normalizing function satisfying $0<$ 
$N_{F} \leq \infty$. (We allow infinity to include the possibility of totally ignoring copies of some $F$. In fact, $N_{F}=n^{|F|+1}$ will do just as well.) Then we may define a subgraph metric associated to $\mathbf{N}=\left(N_{F}\right)_{F \in \mathcal{F}}$ by modifying the definition of $d_{\text {sub }}$ given in Section 3, using the normalized count $\operatorname{emb}(F, G) / N_{F}(|G|)$ in place of $s_{p}(F, G)$. This metric will only make sense for suitably restricted families of graphs, but for such families, it will make much better sense than $d_{\text {sub }}$.

\section{Closing Remarks}

The main aim of this paper is to draw attention to the possibility that there is a rich theory of sparse (quasi-)random graphs waiting to be explored. The beginnings of such a theory can be found in the papers of Bollobás, Janson and Riordan [10, 11] in the very sparse case, and of Borgs, Chayes, Lovász, Sós, Szegedy and Vesztergombi [15, 16, 35] in the dense case; it would be desirable to build a theory encompassing these two extreme threads. As we have just shown, this task is unlikely to be easy: there are numerous unexpected difficulties and pitfalls, and much work has to be done even to arrive at concrete problems whose solutions would represent genuine progress in this endeavour. In this paper we have attempted to do some of this groundwork, and have identified some intriguing problems. As the paper is rather long and, due to the nature of the topic, somewhat diffuse, it may be beneficial to recap some of the results and draw attention to some of the problems and conjectures.

In [10, Bollobás, Janson and Riordan constructed a very general model of an inhomogeneous random graph $G(n, \kappa)$ with $n$ vertices and $\Theta(n)$ edges, where the kernel $\kappa$ is a symmetric measurable function $[0,1]^{2} \rightarrow[0,1]$. As one of the aims of constructing these inhomogeneous random graphs was to model largescale real-world graphs, we should like to be able to say whether or not $G(n, \kappa)$ is a good model for a certain graph $G_{n}$. A little more precisely, we should like to measure the 'distance' between $G_{n}$ and $G(n, \kappa)$, or, simply, $\kappa$, in a sensible way. This leads us to the problem of defining a metric $d$ on the set of graphs such that if $\left(G_{n}\right)$ is a Cauchy sequence in this metric $d$, then the limit of $\left(G_{n}\right)$ in the completion of the space of graphs with distance function $d$ may be naturally identified with a kernel $\kappa$. In addition, we should like the random graphs $G(n, \kappa)$ to tend to $\kappa$ in the metric $d$, either in probability or with probability 1 .

For dense graphs, this program (and more) was carried out by Borgs, Chayes, Lovász, Sós, Szegedy and Vesztergombi in [15, 35] and a number of other papers: they defined several suitable metrics, and proved that they were equivalent. Perhaps the most fundamental of these metrics was based on subgraph counts, suitably normalized. In these papers, the functions playing the role of kernels were called graphons. Unfortunately, every sequence $\left(G_{n}\right)$ in which $G_{n}$ has $n$ vertices and $o\left(n^{2}\right)$ edges converges to the zero graphon. In view of this, one needs some different metrics, metrics that are good at distinguishing sparse graphs. Roughly speaking, a graph $G_{n}$ with $n$ vertices and $p n^{2}\left(\right.$ or $\left.p\left(\begin{array}{l}n \\ 2\end{array}\right)\right)$ edges should be compared to the classical random graph $G_{n, p}$ and its inhomogeneous variants rather than to the complete graph $K_{n}$. This very simple idea gives rise 
to natural extensions of the metrics used by Borgs et al depending on a 'probability function' $p=p(n)$; properties of these metrics are discussed throughout this paper. The idea of comparing a graph to a random graph with about the same number of edges had been used earlier by Kohayakawa and Rödl [33] and others to extend to the sparse setting the cornerstone of these investigations, Szemerédi's Lemma (see Gerke and Steger [29]).

We have conjectured that if our $p$-normalized subgraph counts are suitably bounded and $p=p(n)$ is not too small then an appropriate Cauchy sequence does converge to a kernel (see Conjectures 3.3 and 3.4). Tantalizingly, we cannot even prove this convergence in just about the simplest case, when we know that the limit has to be a constant kernel (Conjecture 3.9).

Sections 4 and 5 are primarily devoted to the study of two fundamental metrics introduced by Borgs, Chayes, Lovász, Sós and Vesztergombi [15], adapted as above to the sparse (but not too sparse) setting. A sound understanding of the relationship between these two metrics, the count and cut metrics, would bring us much closer to a proper theory of sparse inhomogeneous quasi-random graphs. We have conjectured that under some natural and not too restrictive conditions, these two metrics are equivalent in the sense that if $\left(G_{n}\right)$ is a sequence of graphs that are not 'lumpy' then $G_{n}$ converges to a kernel $\kappa$ in the $p$-cut metric if and only if it converges to $\kappa$ in the $p$-count metric (see Conjecture 5.5). As one of our main results, we have proved that $p$-cut convergence does imply $p$-count convergence provided for a restricted set of subgraph counts, under a mild assumption on the distribution of paths of certain lengths (see Theorems 5.14 and 5.16).

Graphs of linear size are discussed in Section 6. It may well turn out that eventually the theory of these 'extremely sparse' graphs will be most significant: unfortunately, many of the basic results proved in the dense case are not valid in this case, and there are severe difficulties in proving suitable replacements. For example, the least one would like is that a sequence $(G(n, c / n))$ of classical random graphs is a Cauchy sequence in the cut metric with probability 1: somewhat surprisingly, this is not the case. Indeed, two realizations of $G(n, c / n)$ will typically be far apart in this metric (see Theorem 6.4). Even more, there are no non-trivial Cauchy sequences (see Theorem 6.2)! It seems that in the extremely sparse setting, one should consider the partition metric instead of the cut metric (these are equivalent for denser graphs); we conjecture that our inhomogeneous random graphs do behave well with respect to this metric (see Conjecture 6.14), although this is likely to be very hard to prove.

Finally, the subgraph metric (suitably adapted) makes very good sense for graphs of linear size: indeed, convergence in this metric is corresponds to the notion of 'local limit' introduced by Benjamini and Schramm [4] and discussed by Aldous and Steel [2. Once again, however, the most basic question is open: what are the possible limit points? (See Question 6.21) Also, the relationship between the subgraph and partition metrics is far from clear. However, it is clear that even if we understood these metrics completely, neither would provide the satisfactory description of (the completion of) the space of sparse graphs that the cut or count metric does for dense graphs. For this reason we propose a metric 
combining the partition and subgraph metrics (see Subsection 7.1). Although this 'coloured neighbourhood' metric may well be very hard to work with, an understanding of its limit points would provide very detailed information about the structure of the space of extremely sparse graphs.

\section{References}

[1] D. Aldous and R. Lyons, Processes on unimodular random networks, preprint available from http://arXiv.org/abs/math/0603062

[2] D. Aldous and J.M. Steele, The objective method: probabilistic combinatorial optimization and local weak convergence, in Probability on discrete structures, Encyclopaedia Math. Sci. 110, Springer (2004), pp. 1-72.

[3] M. Axenovich, A. Kézdy and R. Martin, On the editing distance of graphs, to appear

[4] I. Benjamini and O. Schramm, Recurrence of distributional limits of finite planar graphs, Electron. J. Probab. 6 (2001), no. 23, 13 pp. (electronic).

[5] A. Bertoni, P. Campadelli and R. Posenato, An upper bound for the maximum cut mean value, in Graph-theoretic Concepts in Computer Science (Berlin, 1997), Lecture Notes in Computer Science 1335, Springer, Berlin, 1997, pp. 78-84.

[6] G.R. Blakley and P. Roy, A Hölder type inequality for symmetric matrices with nonnegative entries, Proc. Amer. Math. Soc. 16 (1965), 1244-1245.

[7] B. Bollobás, The isoperimetric number of random regular graphs, European J. Combin. 9 (1988), 241-244.

[8] B. Bollobás, Linear Analysis, 2nd ed., Cambridge University Press (1999), xii +240 pp.

[9] B. Bollobás, C. Borgs, J.T. Chayes and O. Riordan, Percolation on dense graph sequences, preprint available from http://arXiv.org/abs/math/0701346

[10] B. Bollobás, S. Janson and O. Riordan, The phase transition in inhomogeneous random graphs, Random Structures and Algorithms, 31 (2007), $3-122$.

[11] B. Bollobás, S. Janson and O. Riordan, Sparse random graphs with clustering, in preparation.

[12] B. Bollobás and A. Scott, Max Cut for random graphs with a planted partition, Combin. Probab. Comput. 13 (2004), 451-474. 
[13] R. Boppana, Eigenvalues and graph bisection: An average-case analysis (extended abstract), Proceedings of the 28th Annual Symposium on the Foundations of Computer Science (1987), pp. 280-285.

[14] C. Borgs, J.T. Chayes and L. Lovász, Unique limits of dense graph sequences (in preparation).

[15] C. Borgs, J.T. Chayes, L. Lovász, V.T. Sós and K. Vesztergombi, Counting graph homomorphisms, in Topics in Discrete Mathematics (eds. M. Klazar, J. Kratochvil, M. Loebl, J. Matousek, R. Thomas, P. Valtr), Springer (2006), pp 315-371.

[16] C. Borgs, J.T. Chayes, L. Lovász, V.T. Sós, B. Szegedy and K. Vesztergombi, Graph limits and parameter testing, Proc. 38th ACM Symp. Theory of Computing (2006), 261-270.

[17] C. Borgs, J.T. Chayes, L. Lovász, V.T. Sós and K. Vesztergombi, Convergent sequences of dense graphs I: Subgraph frequencies, metric properties and testing. Preprint, May 2006 (revised Jan 2007).

[18] C. Borgs, J.T. Chayes, L. Lovász, V.T. Sós and K. Vesztergombi, Convergent sequences of dense graphs II: Multiway cuts and statistical physics. Preprint, March 2007.

[19] F. Chung and R. Graham, Sparse quasi-random graphs, Combinatorica 22 (2002), 217-244.

[20] F.R.K. Chung, R.L. Graham and R.M. Wilson, Quasi-random graphs, Combinatorica 9 (1989), 345-362.

[21] A. Coja-Oghlan, A spectral heuristic for bisecting random graphs, Random Structures Algorithms 29 (2006), 351-398.

[22] D. Coppersmith, D. Gamarnik, M.T. Hajiaghayi and G.B. Sorkin, Random MAX SAT, random MAX CUT, and their phase transitions, in Proceedings of the Fourteenth Annual ACM-SIAM Symposium on Discrete Algorithms (Baltimore, MD, 2003), ACM, New York, 2003, pp. 364-373.

[23] D. Coppersmith, D. Gamarnik, M.T. Hajiaghayi and G.B. Sorkin, Random MAX SAT, random MAX CUT, and their phase transitions, Random Structures Algorithms 24 (2004), 502-545.

[24] J. Diaz, M.J. Serna and N.C. Wormald, Bounds on the bisection width for random d-regular graphs, to appear

[25] P. Erdős, On some new inequalities concerning extremal properties of graphs, in Theory of Graphs (Proc. Colloq., Tihany, 1966), Academic Press, New York, 1968, pp. 77-81.

[26] U. Feige, M. Karpinski, and M. Langberg, Improved approximation of MaxCut on graphs of bounded degree, J. Algorithms 43 (2002), 201-219. 
[27] A. Frieze and R. Kannan, Quick approximation to matrices and applications, Combinatorica 19 (1999), 175-220.

[28] D. Gamarnik, T. Nowicki and G. Swirszcz, Maximum weight independent sets and matchings in sparse random graphs. Exact results using the local weak convergence method, Random Structures Algorithms 28 (2006), 76106.

[29] S. Gerke and A. Steger, The sparse regularity lemma and its applications, in Surveys in combinatorics 2005, London Math. Soc. Lecture Notes 327, Cambridge University Press (2005), pp. 227-258.

[30] S. Janson, Rounding of continuous random variables and oscillatory asymptotics, Ann. Probab. 34 (2006), 1807-1826.

[31] R. Karp and M. Sipser, Maximum matchings in sparse random graphs, in Proceedings of the 22nd Annual Symposium on the Foundations of Computer Science (1981), pp. 364-375.

[32] Y. Kohayakawa, Szemerédi's regularity lemma for sparse graphs, in Foundations of computational mathematics (Rio de Janeiro, 1997), Springer (1997), pp. 216-230.

[33] Y. Kohayakawa and V. Rödl, Szemerédi's regularity lemma and quasirandomness, in Recent advances in algorithms and combinatorics, CMS Books Math. 11, Springer, (2003) pp. 289-351

[34] A.V. Kostochka and L.S. Melnikov, On a lower bound for the isoperimetric number of cubic graphs, in Probabilistic Methods in Discrete Mathematics (Petrozavodsk, 1992), Progr. Pure Appl. Discrete Math. 1, VSP, Utrecht, 1993, pp. 251-265.

[35] L. Lovász and B. Szegedy, Limits of dense graph sequences, J. Combin. Theory B 96 (2006), 933-957.

[36] L. Lovász and B. Szegedy, Generalized quasirandom graphs, Preprint.

[37] M.J. Luczak and C. McDiarmid, Bisecting sparse random graphs, Random Structures and Algorithms 18 (2001), 31-38.

[38] R. Lyons, Asymptotic enumeration of spanning trees, Combinatorics, Probability and Computing 14 (2005), 491-522.

[39] M. Simonovits, A method for solving extremal problems in graph theory, stability problems, in Theory of Graphs (Proc. Colloq., Tihany, 1966), Academic Press, New York, 1968, pp. 279-319.

[40] A. Thomason, Pseudo-random graphs, in Proceedings of Random Graphs (M. Karonski, ed.), Poznań, 1985, Annals of Discrete Mathematics, 33 (1987) 307-331. 